Electronic Supporting Information Materials

\title{
Eighteen 5,7-dihalo-8-quinolinol and 2,2'-bipyridine Co(II) complexes as a new class of promising anticancer agents
}

Ting Meng a, Qi-Pin Qin a,b,*, Hua-Hong Zou ${ }^{\text {a, }}{ }^{\text {, }}$, Kai Wang a,c and Fu-Pei Liang a,c,,

${ }^{a}$ State Key Laboratory for the Chemistry and Molecular Engineering of Medicinal Resources, School of Chemistry and Pharmacy, Guangxi Normal University, 15 Yucai Road, Guilin 541004, PRChina.E-mail: gxnuchem@foxmail.com (H.-H.ZZu); fliangoffice@yahoo.com (F.-P. Liang).

${ }^{b}$ Guangxi Key Lab of Agricultural Resources Chemistry and Biotechnology, College of Chemistry and Food Science, Yulin Normal University, 1303 Jiaoyudong Road, Yulin 537000, PR China. qpqin2018@126.com (Q.-P.Qin).

${ }^{c}$ Guangxi Key Laboratory of Electrochemical and Magnetochemical Functional Materials, College of Chemistry and Bioengineering, Guilin University of Technology, Guilin 541004, China. 
Table S1. Crystal data and structure refinement details for Co1.

\begin{tabular}{|c|c|}
\hline Empirical formula & $\mathrm{C}_{30} \mathrm{H}_{20} \mathrm{Cl}_{4} \mathrm{CoN}_{4} \mathrm{O}_{2}$ \\
\hline Formula weight & 669.23 \\
\hline Temperature/K & $100.00(10)$ \\
\hline Crystal system & triclinic \\
\hline Space group & P-1 \\
\hline $\mathrm{a} / \AA$ & $9.5622(2)$ \\
\hline $\mathrm{b} / \AA \AA$ & $12.4574(2)$ \\
\hline $\mathrm{c} / \AA$ & $12.6003(3)$ \\
\hline$\alpha /^{\circ}$ & $88.854(2)$ \\
\hline$\beta /{ }^{\circ}$ & $74.261(2)$ \\
\hline$\gamma /{ }^{\circ}$ & $70.474(2)$ \\
\hline Volume $/ \AA^{3}$ & $1357.46(5)$ \\
\hline Z & 2 \\
\hline$\rho_{\text {calc }} \mathrm{g} / \mathrm{cm}^{3}$ & 1.637 \\
\hline$\mu / \mathrm{mm}^{-1}$ & 1.064 \\
\hline $\mathrm{F}(000)$ & 678.0 \\
\hline Crystal size $/ \mathrm{mm}^{3}$ & $0.25 \times 0.21 \times 0.13$ \\
\hline Radiation & $\operatorname{MoK} \alpha(\lambda=0.71073)$ \\
\hline \multicolumn{2}{|c|}{$2 \Theta$ range for data collection $/{ }^{\circ} 3.48$ to 61.884} \\
\hline Index ranges & $-12 \leq \mathrm{h} \leq 12,-16 \leq \mathrm{k} \leq 17,-17 \leq 1 \leq 15$ \\
\hline Reflections collected & 19274 \\
\hline Independent reflections & $6793\left[\mathrm{R}_{\text {int }}=0.0338, \mathrm{R}_{\text {sigma }}=0.0394\right]$ \\
\hline Data/restraints/parameters & $6793 / 0 / 372$ \\
\hline Goodness-of-fit on $\mathrm{F}^{2}$ & 1.027 \\
\hline Final $R$ indexes $[\mathrm{I}>=2 \sigma(\mathrm{I})]$ & $\mathrm{R}_{1}=0.0335, \mathrm{wR}_{2}=0.0797$ \\
\hline Final $\mathrm{R}$ indexes [all data] & $\mathrm{R}_{1}=0.0414, \mathrm{wR}_{2}=0.0841$ \\
\hline Largest diff. peak/hole / e $\AA$ & $0.44 /-0.38$ \\
\hline
\end{tabular}

${ }^{\mathrm{a}} R_{1}=\Sigma|| F_{\mathrm{o}}|-| F_{\mathrm{c}}|| \Sigma\left|F_{\mathrm{o}}\right| ;{ }^{\mathrm{b}} w R_{2}=\left[\Sigma w\left(F_{\mathrm{o}}{ }^{2}-F_{\mathrm{c}}{ }^{2}\right)^{2} / \Sigma w\left(F_{\mathrm{o}}{ }^{2}\right)^{2}\right]^{1 / 2}$

Table S2. Selected bond lengths $(\AA)$ for Co1.

\begin{tabular}{|c|c|c|c|}
\hline Atom Atom & Length/Å & Atom Atom & Length $/ \AA ̊$ \\
\hline $\mathrm{Col}$ & $2.0124(12)$ & C6 & $1.424(2)$ \\
\hline Col & $2.0241(12)$ & $\mathrm{C} 4$ & $1.412(3)$ \\
\hline Col N2 & $2.2080(15)$ & $\mathrm{C} 10$ & $1.413(2)$ \\
\hline
\end{tabular}




\begin{tabular}{cccccc}
\hline Co1 & $\mathrm{N} 1$ & $2.2422(14)$ & $\mathrm{C} 2$ & $\mathrm{C} 3$ & $1.416(2)$ \\
$\mathrm{C} 1$ & $\mathrm{~N} 4$ & $2.1466(16)$ & $\mathrm{C} 2$ & $\mathrm{C} 1$ & $1.497(2)$ \\
$\mathrm{C} 11$ & $\mathrm{~N} 3$ & $2.1498(15)$ & $\mathrm{C} 3$ & $\mathrm{C} 4$ & $1.362(3)$ \\
$\mathrm{C} 12$ & $\mathrm{C} 8$ & $1.7358(18)$ & $\mathrm{C} 19$ & $\mathrm{C} 20$ & $1.369(3)$ \\
$\mathrm{C} 13$ & $\mathrm{C} 18$ & $1.7410(18)$ & $\mathrm{C} 19$ & $\mathrm{C} 18$ & $1.393(3)$ \\
$\mathrm{C} 11$ & $\mathrm{C} 10$ & $1.7455(18)$ & $\mathrm{C} 15$ & $\mathrm{C} 20$ & $1.410(2)$ \\
$\mathrm{C} 14$ & $\mathrm{C} 20$ & $1.7470(18)$ & $\mathrm{C} 15$ & $\mathrm{C} 14$ & $1.416(3)$ \\
$\mathrm{O} 1$ & $\mathrm{C} 7$ & $1.294(2)$ & $\mathrm{C} 12$ & $\mathrm{C} 13$ & $1.419(3)$ \\
$\mathrm{O} 2$ & $\mathrm{C} 17$ & $1.293(2)$ & $\mathrm{C} 12$ & $\mathrm{C} 11$ & $1.500(2)$ \\
$\mathrm{N} 2$ & $\mathrm{C} 16$ & $1.376(2)$ & $\mathrm{C} 25$ & $\mathrm{C} 26$ & $1.488(3)$ \\
$\mathrm{N} 2$ & $\mathrm{C} 12$ & $1.331(2)$ & $\mathrm{C} 25$ & $\mathrm{C} 24$ & $1.395(3)$ \\
$\mathrm{N} 1$ & $\mathrm{C} 2$ & $1.332(2)$ & $\mathrm{C} 26$ & $\mathrm{C} 27$ & $1.388(3)$ \\
$\mathrm{N} 1$ & $\mathrm{C} 6$ & $1.375(2)$ & $\mathrm{C} 8$ & $\mathrm{C} 9$ & $1.400(2)$ \\
$\mathrm{N} 4$ & $\mathrm{C} 26$ & $1.350(2)$ & $\mathrm{C} 14$ & $\mathrm{C} 13$ & $1.359(3)$ \\
$\mathrm{N} 4$ & $\mathrm{C} 30$ & $1.333(2)$ & $\mathrm{C} 10$ & $\mathrm{C} 9$ & $1.362(3)$ \\
$\mathrm{N} 3$ & $\mathrm{C} 25$ & $1.343(2)$ & $\mathrm{C} 30$ & $\mathrm{C} 29$ & $1.390(3)$ \\
$\mathrm{N} 3$ & $\mathrm{C} 21$ & $1.338(3)$ & $\mathrm{C} 27$ & $\mathrm{C} 28$ & $1.380(3)$ \\
$\mathrm{C} 17$ & $\mathrm{C} 16$ & $1.447(2)$ & $\mathrm{C} 21$ & $\mathrm{C} 22$ & $1.385(3)$ \\
$\mathrm{C} 17$ & $\mathrm{C} 18$ & $1.399(2)$ & $\mathrm{C} 29$ & $\mathrm{C} 28$ & $1.383(3)$ \\
$\mathrm{C} 16$ & $\mathrm{C} 15$ & $1.416(3)$ & $\mathrm{C} 24$ & $\mathrm{C} 23$ & $1.383(3)$ \\
$\mathrm{C} 7$ & $\mathrm{C} 6$ & $1.445(2)$ & $\mathrm{C} 23$ & $\mathrm{C} 22$ & $1.377(3)$ \\
$\mathrm{C} 7$ & $\mathrm{C} 8$ & $1.396(2)$ & & & \\
\hline
\end{tabular}

Table S3. Selected bond angles $\left({ }^{\circ}\right)$ for Co1.

\begin{tabular}{cccccccc}
\hline \multicolumn{2}{c}{ Atom Atom } & Atom & Angle $^{\circ}$ & \multicolumn{2}{c}{ Atom Atom Atom } & Angle $^{\circ}$ \\
\hline O1 & Co1 & O2 & $176.40(5)$ & C3 & C2 & C1 & $119.55(16)$ \\
O1 & Co1 & N2 & $104.44(5)$ & N1 & C6 & C7 & $115.87(14)$ \\
O1 & Co1 & N1 & $78.78(5)$ & N1 & C6 & C5 & $122.34(15)$ \\
O1 & Co1 & N4 & $88.06(5)$ & C5 & C6 & C7 & $121.79(15)$ \\
O1 & Co1 & N3 & $87.66(5)$ & C4 & C3 & C2 & $120.69(17)$ \\
O2 & Co1 & N2 & $79.16(5)$ & C3 & C4 & C5 & $119.56(16)$ \\
O2 & Co1 & N1 & $101.38(5)$ & C20 & C19 & C18 & $119.52(16)$ \\
O2 & Co1 & N4 & $88.37(5)$ & C16 & C15 & C14 & $116.94(16)$ \\
O2 & Co1 & N3 & $91.89(5)$ & C20 & C15 & C16 & $118.16(16)$ \\
N2 & Co1 & N1 & $92.07(5)$ & C20 & C15 & C14 & $124.85(17)$ \\
N4 & Co1 & N2 & $165.00(5)$ & N2 & C12 & C13 & $120.94(16)$ \\
N4 & Co1 & N1 & $98.60(6)$ & N2 & C12 & C11 & $119.91(16)$ \\
N4 & Co1 & N3 & $76.65(6)$ & C13 & C12 & C11 & $119.14(16)$ \\
N3 & Co1 & N2 & $95.31(6)$ & N3 & C25 & C26 & $115.86(16)$ \\
\hline
\end{tabular}




\begin{tabular}{cccccccc}
\hline $\mathrm{N} 3$ & $\mathrm{C} 1$ & $\mathrm{~N} 1$ & $165.83(6)$ & $\mathrm{N} 3$ & $\mathrm{C} 25$ & $\mathrm{C} 24$ & $121.04(19)$ \\
$\mathrm{C} 7$ & $\mathrm{O} 1$ & $\mathrm{C} 1$ & $116.34(11)$ & $\mathrm{C} 24$ & $\mathrm{C} 25$ & $\mathrm{C} 26$ & $123.09(19)$ \\
$\mathrm{C} 17$ & $\mathrm{O} 2$ & $\mathrm{C} 1$ & $115.57(10)$ & $\mathrm{N} 4$ & $\mathrm{C} 26$ & $\mathrm{C} 25$ & $115.73(16)$ \\
$\mathrm{C} 16$ & $\mathrm{~N} 2$ & $\mathrm{C} 1$ & $109.13(11)$ & $\mathrm{N} 4$ & $\mathrm{C} 26$ & $\mathrm{C} 27$ & $121.60(18)$ \\
$\mathrm{C} 12$ & $\mathrm{~N} 2$ & $\mathrm{C} 1$ & $131.83(12)$ & $\mathrm{C} 27$ & $\mathrm{C} 26$ & $\mathrm{C} 25$ & $122.63(17)$ \\
$\mathrm{C} 12$ & $\mathrm{~N} 2$ & $\mathrm{C} 16$ & $119.00(15)$ & $\mathrm{C} 19$ & $\mathrm{C} 20$ & $\mathrm{C} 14$ & $118.73(14)$ \\
$\mathrm{C} 2$ & $\mathrm{~N} 1$ & $\mathrm{C} 1$ & $132.76(12)$ & $\mathrm{C} 19$ & $\mathrm{C} 20$ & $\mathrm{C} 15$ & $121.48(16)$ \\
$\mathrm{C} 2$ & $\mathrm{~N} 1$ & $\mathrm{C} 6$ & $118.91(14)$ & $\mathrm{C} 15$ & $\mathrm{C} 20$ & $\mathrm{C} 14$ & $119.78(14)$ \\
$\mathrm{C} 6$ & $\mathrm{~N} 1$ & $\mathrm{C} 1$ & $108.33(11)$ & $\mathrm{C} 7$ & $\mathrm{C} 8$ & $\mathrm{C} 12$ & $118.50(13)$ \\
$\mathrm{C} 26$ & $\mathrm{~N} 4$ & $\mathrm{C} 1$ & $115.59(13)$ & $\mathrm{C} 7$ & $\mathrm{C} 8$ & $\mathrm{C} 9$ & $123.39(16)$ \\
$\mathrm{C} 30$ & $\mathrm{~N} 4$ & $\mathrm{C} 1$ & $125.77(12)$ & $\mathrm{C} 9$ & $\mathrm{C} 8$ & $\mathrm{C} 12$ & $118.05(13)$ \\
$\mathrm{C} 30$ & $\mathrm{~N} 4$ & $\mathrm{C} 26$ & $118.63(16)$ & $\mathrm{C} 17$ & $\mathrm{C} 18$ & $\mathrm{C} 13$ & $118.11(14)$ \\
$\mathrm{C} 25$ & $\mathrm{~N} 3$ & $\mathrm{C} 11$ & $115.41(12)$ & $\mathrm{C} 19$ & $\mathrm{C} 18$ & $\mathrm{C} 13$ & $118.18(13)$ \\
$\mathrm{C} 21$ & $\mathrm{~N} 3$ & $\mathrm{C} 11$ & $125.25(13)$ & $\mathrm{C} 19$ & $\mathrm{C} 18$ & $\mathrm{C} 17$ & $123.69(16)$ \\
$\mathrm{C} 21$ & $\mathrm{~N} 3$ & $\mathrm{C} 25$ & $119.02(16)$ & $\mathrm{C} 13$ & $\mathrm{C} 14$ & $\mathrm{C} 15$ & $119.50(17)$ \\
$\mathrm{O} 2$ & $\mathrm{C} 17$ & $\mathrm{C} 16$ & $120.52(15)$ & $\mathrm{C} 5$ & $\mathrm{C} 10$ & $\mathrm{C} 11$ & $119.29(14)$ \\
$\mathrm{O} 2$ & $\mathrm{C} 17$ & $\mathrm{C} 18$ & $124.16(16)$ & $\mathrm{C} 9$ & $\mathrm{C} 10$ & $\mathrm{C} 11$ & $119.14(14)$ \\
$\mathrm{C} 18$ & $\mathrm{C} 17$ & $\mathrm{C} 16$ & $115.28(16)$ & $\mathrm{C} 9$ & $\mathrm{C} 10$ & $\mathrm{C} 5$ & $121.53(16)$ \\
$\mathrm{N} 2$ & $\mathrm{C} 16$ & $\mathrm{C} 17$ & $115.53(15)$ & $\mathrm{N} 4$ & $\mathrm{C} 30$ & $\mathrm{C} 29$ & $123.03(18)$ \\
$\mathrm{N} 2$ & $\mathrm{C} 16$ & $\mathrm{C} 15$ & $122.66(16)$ & $\mathrm{C} 14$ & $\mathrm{C} 13$ & $\mathrm{C} 12$ & $120.94(17)$ \\
$\mathrm{C} 15$ & $\mathrm{C} 16$ & $\mathrm{C} 17$ & $121.80(15)$ & $\mathrm{C} 10$ & $\mathrm{C} 9$ & $\mathrm{C} 8$ & $119.83(16)$ \\
$\mathrm{O} 1$ & $\mathrm{C} 7$ & $\mathrm{C} 6$ & $120.56(15)$ & $\mathrm{C} 28$ & $\mathrm{C} 27$ & $\mathrm{C} 26$ & $119.13(19)$ \\
$\mathrm{O} 1$ & $\mathrm{C} 7$ & $\mathrm{C} 8$ & $123.91(16)$ & $\mathrm{N} 3$ & $\mathrm{C} 21$ & $\mathrm{C} 22$ & $123.1(2)$ \\
$\mathrm{C} 8$ & $\mathrm{C} 7$ & $\mathrm{C} 6$ & $115.53(15)$ & $\mathrm{C} 28$ & $\mathrm{C} 29$ & $\mathrm{C} 30$ & $118.0(2)$ \\
$\mathrm{C} 4$ & $\mathrm{C} 5$ & $\mathrm{C} 6$ & $117.12(16)$ & $\mathrm{C} 23$ & $\mathrm{C} 24$ & $\mathrm{C} 25$ & $119.1(2)$ \\
$\mathrm{C} 4$ & $\mathrm{C} 5$ & $\mathrm{C} 10$ & $125.00(16)$ & $\mathrm{C} 27$ & $\mathrm{C} 28$ & $\mathrm{C} 29$ & $119.55(19)$ \\
$\mathrm{C} 10$ & $\mathrm{C} 5$ & $\mathrm{C} 6$ & $117.86(16)$ & $\mathrm{C} 22$ & $\mathrm{C} 23$ & $\mathrm{C} 24$ & $119.83(19)$ \\
$\mathrm{N} 1$ & $\mathrm{C} 2$ & $\mathrm{C} 3$ & $121.36(16)$ & $\mathrm{C} 23$ & $\mathrm{C} 22$ & $\mathrm{C} 21$ & $117.8(2)$ \\
$\mathrm{N} 1$ & $\mathrm{C} 2$ & $\mathrm{C} 1$ & $119.08(15)$ & & & & \\
\hline & & & & & & & \\
\end{tabular}

Table S4. Crystal data and structure refinement details for Co2.

\begin{tabular}{ll}
\hline Empirical formula & $\mathrm{C}_{30} \mathrm{H}_{20} \mathrm{Br}_{4} \mathrm{CoN}_{4} \mathrm{O}_{2}$ \\
Formula weight & 847.07 \\
Temperature/K & $293(2)$ \\
Crystal system & triclinic \\
Space group & $\mathrm{P}-1$ \\
$\mathrm{a} / \AA$ & $9.7797(2)$ \\
$\mathrm{b} / \AA$ & $12.6008(3)$ \\
$\mathrm{c} / \AA$ & $12.8998(3)$ \\
$\alpha /{ }^{\circ}$ & $91.024(2)$ \\
$\beta /{ }^{\circ}$ & $110.779(2)$ \\
$\gamma /{ }^{\circ}$ & $104.316(2)$ \\
\hline
\end{tabular}




\begin{tabular}{ll}
\hline Volume $/ \AA^{3}$ & $1430.30(6)$ \\
$\mathrm{Z}$ & 2 \\
$\rho_{\text {calc }} / \mathrm{cm}^{3}$ & 1.967 \\
$\mu / \mathrm{mm}^{-1}$ & 6.224 \\
$\mathrm{~F}(000)$ & 822.0 \\
Crystal size $/ \mathrm{mm}^{3}$ & $0.28 \times 0.21 \times 0.14$ \\
Radiation & $\mathrm{MoK} \alpha(\lambda=0.71073)$ \\
$2 \Theta$ range for data collection/ & 3.4 to 61.442 \\
Index ranges & $-12 \leq \mathrm{h} \leq 13,-16 \leq \mathrm{k} \leq 17,-18 \leq 1 \leq 18$ \\
Reflections collected & 22137 \\
Independent reflections & $7173\left[\mathrm{R}_{\text {int }}=0.0456, \mathrm{R}_{\text {sigma }}=0.0571\right]$ \\
Data/restraints/parameters & $7173 / 0 / 372$ \\
Goodness-of-fit on F & 1.042 \\
Final R indexes [I $>=2 \sigma(\mathrm{I})]$ & $\mathrm{R}_{1}=0.0484, \mathrm{wR}_{2}=0.1004$ \\
Final R indexes [all data] & $\mathrm{R}_{1}=0.0718, \mathrm{wR}_{2}=0.1072$ \\
Largest diff. peak/hole / e $\AA^{-3} 1.33 /-0.63$ \\
\hline
\end{tabular}

${ }^{\mathrm{a}} R_{1}=\Sigma|| F_{\mathrm{o}}|-| F_{\mathrm{c}}|| \Sigma\left|F_{\mathrm{o}}\right| ;{ }^{\mathrm{b}} w R_{2}=\left[\Sigma w\left(F_{\mathrm{o}}{ }^{2}-F_{\mathrm{c}}{ }^{2}\right)^{2} / \Sigma w\left(F_{\mathrm{o}}{ }^{2}\right)^{2}\right]^{1 / 2}$

Table S5. Selected bond lengths (Å) for Co2.

\begin{tabular}{|c|c|c|c|c|c|}
\hline \multicolumn{2}{|c|}{ Atom Atom } & \multirow{2}{*}{$\frac{\text { Length/§̊ }}{1.890(4)}$} & \multicolumn{2}{|c|}{ Atom Atom } & \multirow{2}{*}{$\frac{\text { Length/Å }}{1.344(6)}$} \\
\hline $\mathrm{Br} 2$ & $\mathrm{C} 8$ & & N4 & $\mathrm{C} 30$ & \\
\hline $\mathrm{Br} 3$ & $\mathrm{C} 18$ & $1.895(4)$ & $\mathrm{C} 17$ & $\mathrm{C} 18$ & $1.393(5)$ \\
\hline $\mathrm{Br} 1$ & $\mathrm{C} 10$ & $1.899(4)$ & $\mathrm{C} 17$ & $\mathrm{C} 16$ & $1.453(5)$ \\
\hline $\mathrm{Br} 4$ & $\mathrm{C} 20$ & $1.906(4)$ & C18 & C19 & $1.390(6)$ \\
\hline Col & $\mathrm{O} 1$ & $2.012(3)$ & $\mathrm{C} 12$ & $\mathrm{C} 13$ & $1.413(6)$ \\
\hline Col & $\mathrm{O} 2$ & $2.023(3)$ & $\mathrm{C} 12$ & $\mathrm{C} 11$ & $1.505(5)$ \\
\hline Col & N1 & $2.247(3)$ & $\mathrm{C} 15$ & C14 & $1.421(5)$ \\
\hline Col & N3 & $2.146(3)$ & $\mathrm{C} 15$ & $\mathrm{C} 16$ & $1.413(5)$ \\
\hline Col & $\mathrm{N} 2$ & $2.204(3)$ & $\mathrm{C} 8$ & C9 & $1.397(6)$ \\
\hline Col & N4 & $2.139(4)$ & $\mathrm{C} 8$ & $\mathrm{C} 7$ & $1.398(5)$ \\
\hline $\mathrm{O} 1$ & $\mathrm{C} 7$ & $1.296(5)$ & C14 & $\mathrm{C} 13$ & $1.367(6)$ \\
\hline $\mathrm{O} 2$ & $\mathrm{C} 17$ & $1.295(4)$ & C6 & $\mathrm{C} 7$ & $1.432(6)$ \\
\hline N1 & C6 & $1.380(5)$ & $\mathrm{C} 25$ & $\mathrm{C} 24$ & $1.386(6)$ \\
\hline N1 & $\mathrm{C} 2$ & $1.330(5)$ & $\mathrm{C} 25$ & $\mathrm{C} 26$ & $1.491(6)$ \\
\hline N3 & $\mathrm{C} 25$ & $1.338(5)$ & $\mathrm{C} 24$ & $\mathrm{C} 23$ & $1.381(7)$ \\
\hline N3 & $\mathrm{C} 21$ & $1.331(6)$ & $\mathrm{C} 2$ & $\mathrm{C} 1$ & $1.499(5)$ \\
\hline $\mathrm{N} 2$ & $\mathrm{C} 12$ & $1.331(5)$ & $\mathrm{C} 2$ & $\mathrm{C} 3$ & $1.402(6)$ \\
\hline N2 & $\mathrm{C} 16$ & $1.382(5)$ & $\mathrm{C} 26$ & $\mathrm{C} 27$ & $1.393(6)$ \\
\hline $\mathrm{C} 10$ & $\mathrm{C} 5$ & $1.418(5)$ & $\mathrm{C} 4$ & $\mathrm{C} 3$ & $1.366(5)$ \\
\hline $\mathrm{C} 10$ & C9 & $1.367(6)$ & $\mathrm{C} 27$ & $\mathrm{C} 28$ & $1.388(7)$ \\
\hline $\mathrm{C} 5$ & C6 & $1.424(5)$ & $\mathrm{C} 23$ & $\mathrm{C} 22$ & $1.380(7)$ \\
\hline
\end{tabular}




\begin{tabular}{cccccc}
\hline $\mathrm{C} 5$ & $\mathrm{C} 4$ & $1.412(6)$ & $\mathrm{C} 30$ & $\mathrm{C} 29$ & $1.396(7)$ \\
$\mathrm{C} 20$ & $\mathrm{C} 15$ & $1.406(5)$ & $\mathrm{C} 22$ & $\mathrm{C} 21$ & $1.392(6)$ \\
$\mathrm{C} 20$ & $\mathrm{C} 19$ & $1.376(6)$ & $\mathrm{C} 29$ & $\mathrm{C} 28$ & $1.383(8)$ \\
$\mathrm{N} 4$ & $\mathrm{C} 26$ & $1.352(5)$ & & & \\
\hline
\end{tabular}

Table S6. Selected bond angles ( ${ }^{\circ}$ ) for $\mathbf{C o 2}$.

\begin{tabular}{|c|c|c|c|c|c|c|c|}
\hline \multicolumn{3}{|c|}{ Atom Atom Atom } & \multirow{2}{*}{$\frac{\text { Angle }^{\circ}}{176.80(11)}$} & \multicolumn{3}{|c|}{ Atom Atom Atom } & \multirow{2}{*}{$\frac{\text { Angle }^{\circ}}{123.8(4)}$} \\
\hline $\mathrm{O} 1$ & $\mathrm{Co} 1$ & $\mathrm{O} 2$ & & $\mathrm{C} 19$ & $\mathrm{C} 18$ & $\mathrm{C} 17$ & \\
\hline O1 & Col & N1 & $78.80(12)$ & $\mathrm{N} 2$ & $\mathrm{C} 12$ & $\mathrm{C} 13$ & $121.2(4)$ \\
\hline O1 & $\mathrm{Co} 1$ & N3 & $88.45(11)$ & N2 & $\mathrm{C} 12$ & $\mathrm{C} 11$ & $119.8(3)$ \\
\hline O1 & $\mathrm{Co} 1$ & $\mathrm{~N} 2$ & $104.08(11)$ & $\mathrm{C} 13$ & $\mathrm{C} 12$ & C11 & $119.0(3)$ \\
\hline O1 & Col & N4 & $87.03(12)$ & $\mathrm{C} 20$ & $\mathrm{C} 15$ & $\mathrm{C} 14$ & $124.9(4)$ \\
\hline $\mathrm{O} 2$ & Col & N1 & $101.05(12)$ & $\mathrm{C} 20$ & $\mathrm{C} 15$ & $\mathrm{C} 16$ & $118.2(3)$ \\
\hline $\mathrm{O} 2$ & $\mathrm{Co} 1$ & N3 & $88.41(11)$ & $\mathrm{C} 16$ & $\mathrm{C} 15$ & $\mathrm{C} 14$ & $116.9(4)$ \\
\hline $\mathrm{O} 2$ & $\mathrm{Co} 1$ & $\mathrm{~N} 2$ & $79.12(11)$ & C9 & $\mathrm{C} 8$ & $\mathrm{Br} 2$ & $118.2(3)$ \\
\hline $\mathrm{O} 2$ & Col & N4 & $92.82(12)$ & C9 & $\mathrm{C} 8$ & C7 & $123.1(4)$ \\
\hline N3 & $\mathrm{Co} 1$ & N1 & $97.91(12)$ & C7 & $\mathrm{C} 8$ & $\mathrm{Br} 2$ & $118.6(3)$ \\
\hline N3 & Col & $\mathrm{N} 2$ & $164.57(12)$ & $\mathrm{C} 13$ & $\mathrm{C} 14$ & $\mathrm{C} 15$ & $119.4(4)$ \\
\hline N2 & Col & N1 & $93.41(12)$ & N1 & $\mathrm{C} 6$ & $\mathrm{C} 5$ & $121.7(4)$ \\
\hline N4 & $\mathrm{Co} 1$ & N1 & $164.98(12)$ & N1 & C6 & C7 & $116.5(3)$ \\
\hline N4 & Col & N3 & $76.55(14)$ & C5 & $\mathrm{C} 6$ & C7 & $121.8(3)$ \\
\hline N4 & Col & $\mathrm{N} 2$ & $94.86(13)$ & $\mathrm{C} 10$ & C9 & $\mathrm{C} 8$ & $120.2(4)$ \\
\hline $\mathrm{C} 7$ & $\mathrm{O} 1$ & Co1 & $116.2(2)$ & $\mathrm{C} 14$ & $\mathrm{C} 13$ & $\mathrm{C} 12$ & $120.9(4)$ \\
\hline $\mathrm{C} 17$ & $\mathrm{O} 2$ & $\mathrm{Co} 1$ & 116.1(2) & $\mathrm{C} 20$ & $\mathrm{C} 19$ & $\mathrm{C} 18$ & $119.6(4)$ \\
\hline C6 & N1 & Co1 & $107.6(2)$ & N3 & $\mathrm{C} 25$ & $\mathrm{C} 24$ & $121.7(4)$ \\
\hline $\mathrm{C} 2$ & N1 & Co1 & $133.0(3)$ & N3 & $\mathrm{C} 25$ & $\mathrm{C} 26$ & $115.8(4)$ \\
\hline $\mathrm{C} 2$ & N1 & C6 & 119.4(3) & $\mathrm{C} 24$ & $\mathrm{C} 25$ & $\mathrm{C} 26$ & $122.4(4)$ \\
\hline $\mathrm{C} 25$ & N3 & Co1 & $115.9(3)$ & N2 & $\mathrm{C} 16$ & $\mathrm{C} 17$ & $115.3(3)$ \\
\hline $\mathrm{C} 21$ & N3 & Co1 & $125.2(3)$ & N2 & $\mathrm{C} 16$ & $\mathrm{C} 15$ & $122.8(3)$ \\
\hline $\mathrm{C} 21$ & N3 & $\mathrm{C} 25$ & $118.8(4)$ & $\mathrm{C} 15$ & C16 & $\mathrm{C} 17$ & $121.9(3)$ \\
\hline $\mathrm{C} 12$ & N2 & Co1 & 131.7(3) & O1 & C7 & C8 & $123.5(4)$ \\
\hline $\mathrm{C} 12$ & N2 & C16 & $118.9(3)$ & $\mathrm{O} 1$ & C7 & C6 & $120.6(3)$ \\
\hline $\mathrm{C} 16$ & N2 & Co1 & $109.4(2)$ & C8 & C7 & C6 & $115.9(4)$ \\
\hline $\mathrm{C} 5$ & $\mathrm{C} 10$ & $\mathrm{Br} 1$ & $119.9(3)$ & $\mathrm{C} 23$ & $\mathrm{C} 24$ & $\mathrm{C} 25$ & $119.2(4)$ \\
\hline C9 & $\mathrm{C} 10$ & $\mathrm{Br} 1$ & $119.3(3)$ & N1 & $\mathrm{C} 2$ & $\mathrm{C} 1$ & $118.3(4)$ \\
\hline C9 & $\mathrm{C} 10$ & C5 & $120.8(4)$ & N1 & $\mathrm{C} 2$ & $\mathrm{C} 3$ & $121.6(4)$ \\
\hline $\mathrm{C} 10$ & C5 & C6 & 118.1(4) & C3 & $\mathrm{C} 2$ & $\mathrm{C} 1$ & $120.1(4)$ \\
\hline $\mathrm{C} 4$ & $\mathrm{C} 5$ & $\mathrm{C} 10$ & $124.9(4)$ & N4 & $\mathrm{C} 26$ & $\mathrm{C} 25$ & $115.2(4)$ \\
\hline C4 & $\mathrm{C} 5$ & C6 & $117.0(3)$ & N4 & $\mathrm{C} 26$ & $\mathrm{C} 27$ & $121.6(4)$ \\
\hline $\mathrm{C} 15$ & $\mathrm{C} 20$ & $\mathrm{Br} 4$ & $120.5(3)$ & $\mathrm{C} 27$ & $\mathrm{C} 26$ & $\mathrm{C} 25$ & $123.3(4)$ \\
\hline $\mathrm{C} 19$ & $\mathrm{C} 20$ & $\mathrm{Br} 4$ & $118.3(3)$ & $\mathrm{C} 3$ & $\mathrm{C} 4$ & C5 & $119.9(4)$ \\
\hline
\end{tabular}




\begin{tabular}{cccccccc}
\hline $\mathrm{C} 19$ & $\mathrm{C} 20$ & $\mathrm{C} 15$ & $121.2(4)$ & $\mathrm{C} 4$ & $\mathrm{C} 3$ & $\mathrm{C} 2$ & $120.5(4)$ \\
$\mathrm{C} 26$ & $\mathrm{~N} 4$ & $\mathrm{C} 11$ & $115.6(3)$ & $\mathrm{C} 28$ & $\mathrm{C} 27$ & $\mathrm{C} 26$ & $119.4(5)$ \\
$\mathrm{C} 30$ & $\mathrm{~N} 4$ & $\mathrm{C} 1$ & $125.7(3)$ & $\mathrm{C} 22$ & $\mathrm{C} 23$ & $\mathrm{C} 24$ & $119.4(4)$ \\
$\mathrm{C} 30$ & $\mathrm{~N} 4$ & $\mathrm{C} 26$ & $118.1(4)$ & $\mathrm{N} 4$ & $\mathrm{C} 30$ & $\mathrm{C} 29$ & $123.7(5)$ \\
$\mathrm{O} 2$ & $\mathrm{C} 17$ & $\mathrm{C} 18$ & $124.8(3)$ & $\mathrm{C} 23$ & $\mathrm{C} 22$ & $\mathrm{C} 21$ & $117.9(5)$ \\
$\mathrm{O} 2$ & $\mathrm{C} 17$ & $\mathrm{C} 16$ & $120.0(3)$ & $\mathrm{C} 28$ & $\mathrm{C} 29$ & $\mathrm{C} 30$ & $117.6(5)$ \\
$\mathrm{C} 18$ & $\mathrm{C} 17$ & $\mathrm{C} 16$ & $115.1(3)$ & $\mathrm{N} 3$ & $\mathrm{C} 21$ & $\mathrm{C} 22$ & $123.0(4)$ \\
$\mathrm{C} 17$ & $\mathrm{C} 18$ & $\mathrm{Br} 3$ & $118.1(3)$ & $\mathrm{C} 29$ & $\mathrm{C} 28$ & $\mathrm{C} 27$ & $119.6(5)$ \\
$\mathrm{C} 19$ & $\mathrm{C} 18$ & $\mathrm{Br} 3$ & $118.1(3)$ & & & & \\
\hline
\end{tabular}

Table S7. Crystal data and structure refinement details for Co3.

\begin{tabular}{|c|c|}
\hline Empirical formula & $\mathrm{C}_{32} \mathrm{H}_{20} \mathrm{Cl}_{4} \mathrm{CoN}_{4} \mathrm{O}_{2}$ \\
\hline Formula weight & 693.25 \\
\hline Temperature/K & $100.01(10)$ \\
\hline Crystal system & triclinic \\
\hline Space group & $\mathrm{P}-1$ \\
\hline $\mathrm{a} / \AA$ & $9.8526(6)$ \\
\hline $\mathrm{b} / \AA \AA$ & $12.2886(5)$ \\
\hline $\mathrm{c} / \AA$ & $12.8104(5)$ \\
\hline$\alpha /{ }^{\circ}$ & $87.440(3)$ \\
\hline$\beta /{ }^{\circ}$ & $75.684(4)$ \\
\hline$\gamma /{ }^{\circ}$ & $68.703(5)$ \\
\hline Volume $/ \AA^{3}$ & $1398.46(13)$ \\
\hline Z & 2 \\
\hline$\rho_{\text {calc }} \mathrm{g} / \mathrm{cm}^{3}$ & 1.646 \\
\hline$\mu / \mathrm{mm}^{-1}$ & 1.036 \\
\hline $\mathrm{F}(000)$ & 702.0 \\
\hline Crystal size $/ \mathrm{mm}^{3}$ & $0.25 \times 0.14 \times 0.11$ \\
\hline Radiation & $\operatorname{MoK} \alpha(\lambda=0.71073)$ \\
\hline \multicolumn{2}{|c|}{$2 \Theta$ range for data collection $/{ }^{\circ} 3.562$ to 61.836} \\
\hline Index ranges & $-13 \leq \mathrm{h} \leq 14,-15 \leq \mathrm{k} \leq 16,-17 \leq 1 \leq 17$ \\
\hline Reflections collected & 21112 \\
\hline Independent reflections & $7043\left[\mathrm{R}_{\text {int }}=0.0484, \mathrm{R}_{\text {sigma }}=0.0493\right]$ \\
\hline Data/restraints/parameters & $7043 / 0 / 390$ \\
\hline Goodness-of-fit on $\mathrm{F}^{2}$ & 1.055 \\
\hline Final $\mathrm{R}$ indexes $[\mathrm{I}>=2 \sigma(\mathrm{I})]$ & $\mathrm{R}_{1}=0.0507, \mathrm{wR}_{2}=0.1385$ \\
\hline Final $\mathrm{R}$ indexes [all data] & $\mathrm{R}_{1}=0.0659, \mathrm{wR}_{2}=0.1495$ \\
\hline Largest diff. peak/hole / e $\AA$ & $0.74 /-0.78$ \\
\hline
\end{tabular}

$$
{ }^{\mathrm{a}} R_{1}=\Sigma|| F_{\mathrm{o}}|-| F_{\mathrm{c}}|| \Sigma\left|F_{\mathrm{o}}\right| ;{ }^{\mathrm{b}} w R_{2}=\left[\Sigma w\left(F_{\mathrm{o}}{ }^{2}-F_{\mathrm{c}}{ }^{2}\right)^{2} / \Sigma w\left(F_{\mathrm{o}}\right)^{2}\right]^{1 / 2}
$$


Table S8. Selected bond lengths ( $\AA$ ) for Co3.

\begin{tabular}{|c|c|c|c|c|c|}
\hline \multicolumn{2}{|c|}{ Atom Atom } & \multirow{2}{*}{$\frac{\text { Length/Å }}{2.0364(18)}$} & \multicolumn{2}{|c|}{ Atom Atom } & \multirow{2}{*}{$\frac{\text { Length/Å }}{1.407(4)}$} \\
\hline Col & $\mathrm{O} 2$ & & $\mathrm{C} 12$ & $\mathrm{C} 13$ & \\
\hline Col & $\mathrm{O} 1$ & $2.022(2)$ & $\mathrm{C} 12$ & $\mathrm{C} 11$ & $1.503(4)$ \\
\hline Col & N4 & $2.143(3)$ & $\mathrm{C} 25$ & $\mathrm{C} 24$ & $1.415(4)$ \\
\hline Col & N3 & $2.162(2)$ & C19 & $\mathrm{C} 20$ & $1.372(4)$ \\
\hline $\mathrm{Co} 1$ & $\mathrm{~N} 2$ & $2.209(2)$ & $\mathrm{C} 15$ & $\mathrm{C} 20$ & $1.410(4)$ \\
\hline Col & N1 & $2.217(3)$ & $\mathrm{C} 15$ & C14 & $1.419(4)$ \\
\hline $\mathrm{Cl} 3$ & $\mathrm{C} 18$ & $1.737(3)$ & $\mathrm{C} 29$ & $\mathrm{C} 24$ & $1.428(5)$ \\
\hline $\mathrm{Cl} 2$ & $\mathrm{C} 8$ & $1.735(4)$ & $\mathrm{C} 29$ & $\mathrm{C} 28$ & $1.349(5)$ \\
\hline $\mathrm{Cl} 4$ & $\mathrm{C} 20$ & $1.741(3)$ & C6 & $\mathrm{C} 7$ & $1.421(5)$ \\
\hline $\mathrm{C} 11$ & $\mathrm{C} 10$ & $1.738(3)$ & C6 & $\mathrm{C} 5$ & $1.422(4)$ \\
\hline $\mathrm{O} 2$ & $\mathrm{C} 17$ & $1.288(3)$ & $\mathrm{C} 8$ & $\mathrm{C} 7$ & $1.407(4)$ \\
\hline $\mathrm{O} 1$ & $\mathrm{C} 7$ & $1.276(4)$ & $\mathrm{C} 8$ & $\mathrm{C} 9$ & $1.389(5)$ \\
\hline N4 & $\mathrm{C} 26$ & $1.353(4)$ & $\mathrm{C} 10$ & $\mathrm{C} 5$ & $1.407(4)$ \\
\hline N4 & $\mathrm{C} 32$ & $1.331(4)$ & $\mathrm{C} 10$ & C9 & $1.380(5)$ \\
\hline N3 & $\mathrm{C} 25$ & $1.358(4)$ & $\mathrm{C} 1$ & $\mathrm{C} 2$ & $1.488(4)$ \\
\hline N3 & $\mathrm{C} 21$ & $1.330(4)$ & $\mathrm{C} 32$ & C31 & $1.406(5)$ \\
\hline N2 & $\mathrm{C} 16$ & $1.381(3)$ & $\mathrm{C} 27$ & $\mathrm{C} 28$ & $1.434(4)$ \\
\hline $\mathrm{N} 2$ & $\mathrm{C} 12$ & $1.331(4)$ & $\mathrm{C} 27$ & $\mathrm{C} 30$ & $1.409(5)$ \\
\hline N1 & C6 & $1.396(4)$ & $\mathrm{C} 24$ & $\mathrm{C} 23$ & $1.412(5)$ \\
\hline N1 & $\mathrm{C} 2$ & $1.317(4)$ & $\mathrm{C} 14$ & $\mathrm{C} 13$ & $1.356(4)$ \\
\hline $\mathrm{C} 16$ & $\mathrm{C} 17$ & $1.448(4)$ & $\mathrm{C} 2$ & $\mathrm{C} 3$ & $1.423(4)$ \\
\hline $\mathrm{C} 16$ & $\mathrm{C} 15$ & $1.412(4)$ & $\mathrm{C} 21$ & $\mathrm{C} 22$ & $1.406(4)$ \\
\hline $\mathrm{C} 17$ & C18 & $1.398(4)$ & $\mathrm{C} 5$ & $\mathrm{C} 4$ & $1.404(5)$ \\
\hline $\mathrm{C} 26$ & $\mathrm{C} 25$ & $1.442(4)$ & $\mathrm{C} 3$ & $\mathrm{C} 4$ & $1.367(5)$ \\
\hline $\mathrm{C} 26$ & $\mathrm{C} 27$ & $1.406(4)$ & $\mathrm{C} 23$ & $\mathrm{C} 22$ & $1.355(5)$ \\
\hline C18 & C19 & $1.397(4)$ & $\mathrm{C} 30$ & C31 & $1.362(5)$ \\
\hline
\end{tabular}

Table S9. Selected bond angles $\left(^{\circ}\right)$ for $\mathbf{C o 3}$

\begin{tabular}{|c|c|c|c|c|c|c|c|}
\hline \multicolumn{3}{|c|}{ Atom Atom Atom } & \multirow{2}{*}{$\frac{\text { Angle }^{\circ}}{90.01(8)}$} & \multicolumn{3}{|c|}{ Atom Atom Atom } & \multirow{2}{*}{$\frac{\text { Angle }^{\circ}}{119.2(3)}$} \\
\hline $\mathrm{O} 2$ & Co1 & N4 & & $\mathrm{C} 24$ & $\mathrm{C} 25$ & $\mathrm{C} 26$ & \\
\hline $\mathrm{O} 2$ & Col & N3 & $88.85(8)$ & $\mathrm{C} 20$ & C19 & $\mathrm{C} 18$ & $120.2(3)$ \\
\hline $\mathrm{O} 2$ & Col & $\mathrm{N} 2$ & $78.96(8)$ & $\mathrm{C} 16$ & $\mathrm{C} 15$ & $\mathrm{C} 14$ & $117.1(3)$ \\
\hline $\mathrm{O} 2$ & Col & $\mathrm{N} 1$ & 103.07(9) & $\mathrm{C} 20$ & $\mathrm{C} 15$ & $\mathrm{C} 16$ & $118.4(3)$ \\
\hline $\mathrm{O} 1$ & Col & $\mathrm{O} 2$ & $175.02(8)$ & $\mathrm{C} 20$ & $\mathrm{C} 15$ & $\mathrm{C} 14$ & $124.4(3)$ \\
\hline $\mathrm{O} 1$ & Col & N4 & $87.77(9)$ & $\mathrm{C} 28$ & $\mathrm{C} 29$ & $\mathrm{C} 24$ & $121.3(3)$ \\
\hline $\mathrm{O} 1$ & Co1 & $\mathrm{N} 3$ & $86.33(9)$ & N1 & C6 & $\mathrm{C} 7$ & $115.7(3)$ \\
\hline $\mathrm{O} 1$ & Col & $\mathrm{N} 2$ & $105.62(9)$ & N1 & C6 & C5 & $120.9(3)$ \\
\hline $\mathrm{O} 1$ & Co1 & N1 & 78.91(9) & $\mathrm{C} 7$ & C6 & $\mathrm{C} 5$ & $123.4(3)$ \\
\hline N4 & Col & N3 & $77.58(10)$ & $\mathrm{C} 7$ & $\mathrm{C} 8$ & $\mathrm{Cl} 2$ & $117.8(2)$ \\
\hline
\end{tabular}




\begin{tabular}{|c|c|c|c|c|c|c|c|}
\hline $\mathrm{N} 4$ & Col & $\mathrm{N} 2$ & $93.44(9)$ & C9 & $\mathrm{C} 8$ & $\mathrm{Cl} 2$ & $118.8(2)$ \\
\hline N4 & Col & N1 & $166.44(9)$ & C9 & $\mathrm{C} 8$ & $\mathrm{C} 7$ & $123.3(3)$ \\
\hline N3 & Col & $\mathrm{N} 2$ & $164.92(9)$ & $\mathrm{C} 5$ & $\mathrm{C} 10$ & $\mathrm{Cl1}$ & $120.7(3)$ \\
\hline N3 & Col & N1 & $98.85(9)$ & C9 & $\mathrm{C} 10$ & $\mathrm{Cl1}$ & $118.8(2)$ \\
\hline $\mathrm{N} 2$ & Col & N1 & $92.58(9)$ & C9 & $\mathrm{C} 10$ & $\mathrm{C} 5$ & $120.5(3)$ \\
\hline $\mathrm{C} 17$ & $\mathrm{O} 2$ & Co1 & $115.58(16)$ & N4 & C32 & $\mathrm{C} 31$ & $123.4(3)$ \\
\hline $\mathrm{C} 7$ & $\mathrm{O} 1$ & Col & $115.87(19)$ & C26 & $\mathrm{C} 27$ & $\mathrm{C} 28$ & $119.4(3)$ \\
\hline $\mathrm{C} 26$ & N4 & Co1 & $114.2(2)$ & C26 & $\mathrm{C} 27$ & $\mathrm{C} 30$ & $117.2(3)$ \\
\hline C32 & N4 & Co1 & $128.0(2)$ & C30 & $\mathrm{C} 27$ & $\mathrm{C} 28$ & $123.4(3)$ \\
\hline C32 & N4 & $\mathrm{C} 26$ & $117.8(3)$ & $\mathrm{C} 25$ & $\mathrm{C} 24$ & $\mathrm{C} 29$ & $119.5(3)$ \\
\hline $\mathrm{C} 25$ & N3 & Col & 113.07(19) & $\mathrm{C} 23$ & $\mathrm{C} 24$ & $\mathrm{C} 25$ & $117.0(3)$ \\
\hline $\mathrm{C} 21$ & N3 & Col & $128.9(2)$ & $\mathrm{C} 23$ & $\mathrm{C} 24$ & $\mathrm{C} 29$ & $123.6(3)$ \\
\hline $\mathrm{C} 21$ & N3 & $\mathrm{C} 25$ & $118.0(3)$ & $\mathrm{O} 1$ & $\mathrm{C} 7$ & C6 & $121.4(3)$ \\
\hline $\mathrm{C} 16$ & N2 & Co1 & 109.31(17) & $\mathrm{O} 1$ & $\mathrm{C} 7$ & $\mathrm{C} 8$ & $123.8(3)$ \\
\hline $\mathrm{C} 12$ & N2 & Col & $132.24(19)$ & $\mathrm{C} 8$ & $\mathrm{C} 7$ & C6 & $114.8(3)$ \\
\hline $\mathrm{C} 12$ & N2 & $\mathrm{C} 16$ & $118.4(2)$ & C29 & C28 & $\mathrm{C} 27$ & $121.0(3)$ \\
\hline C6 & N1 & Col & $108.0(2)$ & C19 & $\mathrm{C} 20$ & $\mathrm{Cl} 4$ & $119.4(2)$ \\
\hline $\mathrm{C} 2$ & N1 & Col & $132.45(19)$ & C19 & $\mathrm{C} 20$ & $\mathrm{C} 15$ & $120.8(3)$ \\
\hline $\mathrm{C} 2$ & N1 & C6 & $119.5(3)$ & $\mathrm{C} 15$ & $\mathrm{C} 20$ & $\mathrm{Cl} 4$ & $119.9(2)$ \\
\hline $\mathrm{N} 2$ & $\mathrm{C} 16$ & $\mathrm{C} 17$ & $115.4(2)$ & C13 & C14 & $\mathrm{C} 15$ & $119.5(3)$ \\
\hline $\mathrm{N} 2$ & $\mathrm{C} 16$ & $\mathrm{C} 15$ & $122.5(2)$ & $\mathrm{N} 1$ & $\mathrm{C} 2$ & $\mathrm{C} 1$ & $118.7(3)$ \\
\hline $\mathrm{C} 15$ & $\mathrm{C} 16$ & $\mathrm{C} 17$ & $122.1(2)$ & N1 & $\mathrm{C} 2$ & $\mathrm{C} 3$ & $121.7(3)$ \\
\hline $\mathrm{O} 2$ & C17 & $\mathrm{C} 16$ & $120.7(2)$ & $\mathrm{C} 3$ & $\mathrm{C} 2$ & $\mathrm{C} 1$ & $119.6(3)$ \\
\hline $\mathrm{O} 2$ & C17 & $\mathrm{C} 18$ & $124.1(3)$ & N3 & $\mathrm{C} 21$ & $\mathrm{C} 22$ & $122.6(3)$ \\
\hline C18 & $\mathrm{C} 17$ & $\mathrm{C} 16$ & $115.2(2)$ & $\mathrm{C} 10$ & $\mathrm{C} 5$ & C6 & $117.7(3)$ \\
\hline N4 & C26 & $\mathrm{C} 25$ & $117.3(3)$ & $\mathrm{C} 4$ & C5 & C6 & $118.0(3)$ \\
\hline $\mathrm{N} 4$ & C26 & $\mathrm{C} 27$ & $123.0(3)$ & $\mathrm{C} 4$ & $\mathrm{C} 5$ & $\mathrm{C} 10$ & $124.3(3)$ \\
\hline $\mathrm{C} 27$ & $\mathrm{C} 26$ & $\mathrm{C} 25$ & $119.7(3)$ & $\mathrm{C} 14$ & C13 & $\mathrm{C} 12$ & $120.7(3)$ \\
\hline $\mathrm{C} 17$ & C18 & $\mathrm{Cl} 3$ & $118.4(2)$ & $\mathrm{C} 4$ & $\mathrm{C} 3$ & $\mathrm{C} 2$ & $119.9(3)$ \\
\hline C19 & C18 & $\mathrm{Cl} 3$ & $118.3(2)$ & $\mathrm{C} 10$ & C9 & $\mathrm{C} 8$ & $120.3(3)$ \\
\hline C19 & C18 & $\mathrm{C} 17$ & $123.3(3)$ & $\mathrm{C} 3$ & $\mathrm{C} 4$ & $\mathrm{C} 5$ & $119.9(3)$ \\
\hline $\mathrm{N} 2$ & $\mathrm{C} 12$ & $\mathrm{C} 13$ & $121.7(3)$ & $\mathrm{C} 22$ & $\mathrm{C} 23$ & $\mathrm{C} 24$ & $119.6(3)$ \\
\hline $\mathrm{N} 2$ & $\mathrm{C} 12$ & $\mathrm{C} 11$ & 119.3(3) & $\mathrm{C} 23$ & $\mathrm{C} 22$ & $\mathrm{C} 21$ & $119.8(3)$ \\
\hline $\mathrm{C} 13$ & $\mathrm{C} 12$ & $\mathrm{C} 11$ & $118.9(3)$ & C31 & $\mathrm{C} 30$ & $\mathrm{C} 27$ & $120.2(3)$ \\
\hline N3 & $\mathrm{C} 25$ & $\mathrm{C} 26$ & $117.9(3)$ & C30 & C31 & $\mathrm{C} 32$ & $118.4(3)$ \\
\hline N3 & $\mathrm{C} 25$ & $\mathrm{C} 24$ & $123.0(3)$ & & & & \\
\hline
\end{tabular}


Table S10. Crystal data and structure refinement details for Co4.

\begin{tabular}{|c|c|}
\hline Empirical formula & $\mathrm{C}_{32} \mathrm{H}_{20} \mathrm{Br}_{4} \mathrm{CoN}_{4} \mathrm{O}_{2}$ \\
\hline Formula weight & 871.09 \\
\hline Temperature/K & $100.00(10)$ \\
\hline Crystal system & triclinic \\
\hline Space group & $\mathrm{P}-1$ \\
\hline $\mathrm{a} / \AA$ & $10.2841(4)$ \\
\hline $\mathrm{b} / \AA$ & $12.4309(4)$ \\
\hline $\mathrm{c} / \AA ̊$ & $12.8637(3)$ \\
\hline$\alpha /^{\circ}$ & $80.072(2)$ \\
\hline$\beta /{ }^{\circ}$ & $76.026(2)$ \\
\hline$\gamma /{ }^{\circ}$ & $67.195(3)$ \\
\hline Volume $/ \AA^{3}$ & $1465.37(9)$ \\
\hline Z & 2 \\
\hline$\rho_{\text {calc }} \mathrm{g} / \mathrm{cm}^{3}$ & 1.974 \\
\hline$\mu / \mathrm{mm}^{-1}$ & 6.078 \\
\hline $\mathrm{F}(000)$ & 846.0 \\
\hline Crystal size $/ \mathrm{mm}^{3}$ & $0.25 \times 0.18 \times 0.11$ \\
\hline Radiation & $\operatorname{MoK} \alpha(\lambda=0.71073)$ \\
\hline \multicolumn{2}{|c|}{$2 \Theta$ range for data collection $/{ }^{\circ} 3.568$ to 61.896} \\
\hline Index ranges & $-12 \leq \mathrm{h} \leq 14,-15 \leq \mathrm{k} \leq 16,-17 \leq 1 \leq 17$ \\
\hline Reflections collected & 17766 \\
\hline Independent reflections & $7283\left[\mathrm{R}_{\text {int }}=0.0445, \mathrm{R}_{\text {sigma }}=0.0624\right]$ \\
\hline Data/restraints/parameters & $7283 / 0 / 390$ \\
\hline Goodness-of-fit on $\mathrm{F}^{2}$ & 1.026 \\
\hline Final $R$ indexes $[\mathrm{I}>=2 \sigma(\mathrm{I})]$ & $\mathrm{R}_{1}=0.0526, \mathrm{wR}_{2}=0.1112$ \\
\hline Final $\mathrm{R}$ indexes [all data] & $\mathrm{R}_{1}=0.0846, \mathrm{wR}_{2}=0.1221$ \\
\hline Largest diff. peak/hole / e $\AA$ & $1.50 /-0.85$ \\
\hline
\end{tabular}

Table S11. Selected bond lengths ( $\AA$ ) for Co4.

\begin{tabular}{|c|c|c|c|}
\hline Atom Atom & Length/Å & Atom Atom & Length/Å \\
\hline $\mathrm{Br} 2$ & $1.898(5)$ & C9 & $1.397(7)$ \\
\hline $\mathrm{Br} 3 \quad \mathrm{C} 18$ & $1.892(5)$ & C6 & $1.426(7)$ \\
\hline $\mathrm{Br} 4 \quad \mathrm{C} 20$ & $1.901(5)$ & $\mathrm{C} 4$ & $1.412(7)$ \\
\hline Br1 C10 & $1.914(5)$ & $\mathrm{C} 5 \mathrm{C} 10$ & $1.401(7)$ \\
\hline
\end{tabular}




\begin{tabular}{|c|c|c|c|c|c|}
\hline Col & $\mathrm{O} 1$ & $2.041(3)$ & $\mathrm{C} 15$ & $\mathrm{C} 16$ & $1.420(7)$ \\
\hline Col & $\mathrm{O} 2$ & $2.019(3)$ & C15 & $\mathrm{C} 20$ & $1.405(7)$ \\
\hline Col & N3 & $2.138(4)$ & $\mathrm{C} 15$ & $\mathrm{C} 14$ & $1.420(7)$ \\
\hline Col & N4 & $2.153(4)$ & C32 & C31 & $1.406(8)$ \\
\hline Col & N2 & $2.203(4)$ & C26 & $\mathrm{C} 27$ & $1.417(7)$ \\
\hline Col & N1 & $2.216(4)$ & C9 & $\mathrm{C} 10$ & $1.373(7)$ \\
\hline $\mathrm{O} 1$ & $\mathrm{C} 7$ & $1.287(6)$ & $\mathrm{C} 4$ & $\mathrm{C} 3$ & $1.350(7)$ \\
\hline $\mathrm{O} 2$ & C17 & $1.283(6)$ & $\mathrm{C} 20$ & C19 & $1.375(7)$ \\
\hline N3 & $\mathrm{C} 25$ & $1.349(7)$ & C18 & C19 & $1.380(7)$ \\
\hline $\mathrm{N} 3$ & $\mathrm{C} 21$ & $1.318(7)$ & $\mathrm{C} 12$ & $\mathrm{C} 11$ & $1.490(7)$ \\
\hline $\mathrm{N} 4$ & C32 & $1.325(7)$ & $\mathrm{C} 12$ & $\mathrm{C} 13$ & $1.433(7)$ \\
\hline $\mathrm{N} 4$ & C26 & $1.353(6)$ & $\mathrm{C} 2$ & $\mathrm{C} 3$ & $1.416(7)$ \\
\hline $\mathrm{N} 2$ & $\mathrm{C} 16$ & $1.383(6)$ & $\mathrm{C} 2$ & $\mathrm{C} 1$ & $1.495(7)$ \\
\hline $\mathrm{N} 2$ & $\mathrm{C} 12$ & $1.305(6)$ & $\mathrm{C} 27$ & $\mathrm{C} 30$ & $1.401(8)$ \\
\hline $\mathrm{N} 1$ & C6 & $1.369(6)$ & $\mathrm{C} 27$ & $\mathrm{C} 28$ & $1.441(8)$ \\
\hline $\mathrm{N} 1$ & $\mathrm{C} 2$ & $1.325(7)$ & C31 & $\mathrm{C} 30$ & $1.358(8)$ \\
\hline $\mathrm{C} 7$ & $\mathrm{C} 8$ & $1.398(7)$ & C14 & $\mathrm{C} 13$ & $1.348(7)$ \\
\hline $\mathrm{C} 7$ & C6 & $1.443(7)$ & $\mathrm{C} 24$ & $\mathrm{C} 29$ & $1.428(8)$ \\
\hline $\mathrm{C} 17$ & $\mathrm{C} 16$ & $1.440(7)$ & $\mathrm{C} 24$ & $\mathrm{C} 23$ & $1.403(8)$ \\
\hline $\mathrm{C} 17$ & C18 & $1.409(7)$ & C28 & $\mathrm{C} 29$ & $1.347(8)$ \\
\hline $\mathrm{C} 25$ & $\mathrm{C} 26$ & $1.445(7)$ & $\mathrm{C} 21$ & $\mathrm{C} 22$ & $1.418(8)$ \\
\hline $\mathrm{C} 25$ & C24 & $1.418(7)$ & $\mathrm{C} 23$ & $\mathrm{C} 22$ & $1.365(8)$ \\
\hline
\end{tabular}

Table S12. Selected bond angles $\left(^{\circ}\right)$ for Co4.

\begin{tabular}{|c|c|c|c|c|c|c|c|}
\hline \multicolumn{3}{|c|}{ Atom Atom Atom } & \multirow{2}{*}{$\frac{\text { Angle }^{\circ}}{89.39(15)}$} & \multicolumn{3}{|c|}{ Atom Atom Atom } & \multirow{2}{*}{$\frac{\text { Angle/ }^{\circ}}{116.6(4)}$} \\
\hline $\mathrm{O} 1$ & $\mathrm{Co} 1$ & N3 & & $\mathrm{C} 14$ & $\mathrm{C} 15$ & $\mathrm{C} 16$ & \\
\hline $\mathrm{O} 1$ & Col & N4 & $89.42(14)$ & N4 & C32 & $\mathrm{C} 31$ & $123.2(5)$ \\
\hline $\mathrm{O} 1$ & Col & N2 & $103.60(15)$ & N4 & $\mathrm{C} 26$ & $\mathrm{C} 25$ & $117.3(4)$ \\
\hline $\mathrm{O} 1$ & Col & N1 & $78.59(14)$ & N4 & $\mathrm{C} 26$ & $\mathrm{C} 27$ & $122.8(5)$ \\
\hline $\mathrm{O} 2$ & Col & $\mathrm{O} 1$ & $175.18(14)$ & $\mathrm{C} 27$ & $\mathrm{C} 26$ & $\mathrm{C} 25$ & $119.8(5)$ \\
\hline $\mathrm{O} 2$ & Col & N3 & $87.30(15)$ & $\mathrm{C} 10$ & C9 & $\mathrm{C} 8$ & $119.2(5)$ \\
\hline $\mathrm{O} 2$ & Co1 & N4 & $86.44(15)$ & N1 & C6 & $\mathrm{C} 7$ & $116.1(4)$ \\
\hline $\mathrm{O} 2$ & Col & N2 & $79.43(15)$ & N1 & C6 & $\mathrm{C} 5$ & $121.8(5)$ \\
\hline $\mathrm{O} 2$ & Col & N1 & $105.15(15)$ & $\mathrm{C} 5$ & C6 & $\mathrm{C} 7$ & $122.1(4)$ \\
\hline N3 & Col & N4 & $77.56(16)$ & $\mathrm{N} 2$ & $\mathrm{C} 16$ & $\mathrm{C} 17$ & $116.0(4)$ \\
\hline N3 & Col & N2 & $166.11(15)$ & $\mathrm{N} 2$ & $\mathrm{C} 16$ & $\mathrm{C} 15$ & $121.8(4)$ \\
\hline N3 & Col & N1 & $94.30(15)$ & $\mathrm{C} 15$ & $\mathrm{C} 16$ & $\mathrm{C} 17$ & $122.2(4)$ \\
\hline N4 & Col & N2 & $97.39(15)$ & $\mathrm{C} 3$ & $\mathrm{C} 4$ & $\mathrm{C} 5$ & $120.0(5)$ \\
\hline N4 & Col & N1 & $165.65(15)$ & $\mathrm{C} 15$ & $\mathrm{C} 20$ & $\mathrm{Br} 4$ & $120.4(4)$ \\
\hline $\mathrm{N} 2$ & Col & N1 & $93.11(15)$ & C19 & $\mathrm{C} 20$ & $\mathrm{Br} 4$ & $118.7(4)$ \\
\hline C7 & $\mathrm{O} 1$ & Co1 & $115.7(3)$ & C19 & C20 & $\mathrm{C} 15$ & $120.9(5)$ \\
\hline
\end{tabular}




\begin{tabular}{cccccccc}
\hline $\mathrm{C} 17$ & $\mathrm{O} 2$ & $\mathrm{C} 1$ & $115.6(3)$ & $\mathrm{C} 17$ & $\mathrm{C} 18$ & $\mathrm{Br} 3$ & $117.1(4)$ \\
$\mathrm{C} 25$ & $\mathrm{~N} 3$ & $\mathrm{C} 1$ & $114.1(3)$ & $\mathrm{C} 19$ & $\mathrm{C} 18$ & $\mathrm{Br} 3$ & $119.4(4)$ \\
$\mathrm{C} 21$ & $\mathrm{~N} 3$ & $\mathrm{C} 1$ & $128.1(4)$ & $\mathrm{C} 19$ & $\mathrm{C} 18$ & $\mathrm{C} 17$ & $123.5(5)$ \\
$\mathrm{C} 21$ & $\mathrm{~N} 3$ & $\mathrm{C} 25$ & $117.7(5)$ & $\mathrm{C} 20$ & $\mathrm{C} 19$ & $\mathrm{C} 18$ & $120.3(5)$ \\
$\mathrm{C} 32$ & $\mathrm{~N} 4$ & $\mathrm{C} 1$ & $128.8(4)$ & $\mathrm{N} 2$ & $\mathrm{C} 12$ & $\mathrm{C} 11$ & $119.5(5)$ \\
$\mathrm{C} 32$ & $\mathrm{~N} 4$ & $\mathrm{C} 26$ & $117.6(4)$ & $\mathrm{N} 2$ & $\mathrm{C} 12$ & $\mathrm{C} 13$ & $121.1(4)$ \\
$\mathrm{C} 26$ & $\mathrm{~N} 4$ & $\mathrm{C} 1$ & $113.5(3)$ & $\mathrm{C} 13$ & $\mathrm{C} 12$ & $\mathrm{C} 11$ & $119.4(5)$ \\
$\mathrm{C} 16$ & $\mathrm{~N} 2$ & $\mathrm{C} 1$ & $108.4(3)$ & $\mathrm{C} 5$ & $\mathrm{C} 10$ & $\mathrm{Br} 1$ & $119.8(4)$ \\
$\mathrm{C} 12$ & $\mathrm{~N} 2$ & $\mathrm{C} 1$ & $131.7(3)$ & $\mathrm{C} 9$ & $\mathrm{C} 10$ & $\mathrm{Br} 1$ & $118.1(4)$ \\
$\mathrm{C} 12$ & $\mathrm{~N} 2$ & $\mathrm{C} 16$ & $120.0(4)$ & $\mathrm{C} 9$ & $\mathrm{C} 10$ & $\mathrm{C} 5$ & $122.1(4)$ \\
$\mathrm{C} 6$ & $\mathrm{~N} 1$ & $\mathrm{C} 1$ & $109.2(3)$ & $\mathrm{N} 1$ & $\mathrm{C} 2$ & $\mathrm{C} 3$ & $120.7(5)$ \\
$\mathrm{C} 2$ & $\mathrm{~N} 1$ & $\mathrm{C} 1$ & $131.0(3)$ & $\mathrm{N} 1$ & $\mathrm{C} 2$ & $\mathrm{C} 1$ & $120.2(5)$ \\
$\mathrm{C} 2$ & $\mathrm{~N} 1$ & $\mathrm{C} 6$ & $119.8(4)$ & $\mathrm{C} 3$ & $\mathrm{C} 2$ & $\mathrm{C} 1$ & $119.1(5)$ \\
$\mathrm{O} 1$ & $\mathrm{C} 7$ & $\mathrm{C} 8$ & $124.3(4)$ & $\mathrm{C} 4$ & $\mathrm{C} 3$ & $\mathrm{C} 2$ & $120.9(5)$ \\
$\mathrm{O} 1$ & $\mathrm{C} 7$ & $\mathrm{C} 6$ & $120.4(4)$ & $\mathrm{C} 26$ & $\mathrm{C} 27$ & $\mathrm{C} 28$ & $118.9(5)$ \\
$\mathrm{C} 8$ & $\mathrm{C} 7$ & $\mathrm{C} 6$ & $115.3(4)$ & $\mathrm{C} 30$ & $\mathrm{C} 27$ & $\mathrm{C} 26$ & $117.5(5)$ \\
$\mathrm{O} 2$ & $\mathrm{C} 17$ & $\mathrm{C} 16$ & $120.6(4)$ & $\mathrm{C} 30$ & $\mathrm{C} 27$ & $\mathrm{C} 28$ & $123.6(5)$ \\
$\mathrm{O} 2$ & $\mathrm{C} 17$ & $\mathrm{C} 18$ & $124.5(4)$ & $\mathrm{C} 30$ & $\mathrm{C} 31$ & $\mathrm{C} 32$ & $119.4(5)$ \\
$\mathrm{C} 18$ & $\mathrm{C} 17$ & $\mathrm{C} 16$ & $114.9(4)$ & $\mathrm{C} 13$ & $\mathrm{C} 14$ & $\mathrm{C} 15$ & $120.2(5)$ \\
$\mathrm{N} 3$ & $\mathrm{C} 25$ & $\mathrm{C} 26$ & $117.4(4)$ & $\mathrm{C} 25$ & $\mathrm{C} 24$ & $\mathrm{C} 29$ & $119.5(5)$ \\
$\mathrm{N} 3$ & $\mathrm{C} 25$ & $\mathrm{C} 24$ & $123.4(5)$ & $\mathrm{C} 23$ & $\mathrm{C} 24$ & $\mathrm{C} 25$ & $116.1(5)$ \\
$\mathrm{C} 24$ & $\mathrm{C} 25$ & $\mathrm{C} 26$ & $119.1(5)$ & $\mathrm{C} 23$ & $\mathrm{C} 24$ & $\mathrm{C} 29$ & $124.4(5)$ \\
$\mathrm{C} 7$ & $\mathrm{C} 8$ & $\mathrm{Br} 2$ & $118.9(4)$ & $\mathrm{C} 31$ & $\mathrm{C} 30$ & $\mathrm{C} 27$ & $119.4(5)$ \\
$\mathrm{C} 9$ & $\mathrm{C} 8$ & $\mathrm{Br} 2$ & $117.5(4)$ & $\mathrm{C} 29$ & $\mathrm{C} 28$ & $\mathrm{C} 27$ & $121.1(5)$ \\
$\mathrm{C} 9$ & $\mathrm{C} 8$ & $\mathrm{C} 7$ & $123.6(5)$ & $\mathrm{C} 28$ & $\mathrm{C} 29$ & $\mathrm{C} 24$ & $121.6(5)$ \\
$\mathrm{C} 4$ & $\mathrm{C} 5$ & $\mathrm{C} 6$ & $116.7(4)$ & $\mathrm{C} 14$ & $\mathrm{C} 13$ & $\mathrm{C} 12$ & $120.3(5)$ \\
$\mathrm{C} 10$ & $\mathrm{C} 5$ & $\mathrm{C} 6$ & $117.6(5)$ & $\mathrm{N} 3$ & $\mathrm{C} 21$ & $\mathrm{C} 22$ & $124.1(5)$ \\
$\mathrm{C} 10$ & $\mathrm{C} 5$ & $\mathrm{C} 4$ & $125.6(5)$ & $\mathrm{C} 22$ & $\mathrm{C} 23$ & $\mathrm{C} 24$ & $121.4(5)$ \\
$\mathrm{C} 20$ & $\mathrm{C} 15$ & $\mathrm{C} 16$ & $118.1(4)$ & $\mathrm{C} 23$ & $\mathrm{C} 22$ & $\mathrm{C} 21$ & $117.1(5)$ \\
$\mathrm{C} 20$ & $\mathrm{C} 15$ & $\mathrm{C} 14$ & $125.2(5)$ & & & & \\
\hline
\end{tabular}

Table S13. Crystal data and structure refinement details for Co5.

\begin{tabular}{ll}
\hline Empirical formula & $\mathrm{C}_{38} \mathrm{H}_{36} \mathrm{Cl}_{4} \mathrm{CoN}_{6} \mathrm{O}_{6}$ \\
Formula weight & 873.46 \\
Temperature/K & $100.01(10)$ \\
Crystal system & monoclinic \\
Space group & $\mathrm{P} 2{ }_{1} / \mathrm{n}$ \\
$\mathrm{a} / \AA$ & $13.5561(4)$ \\
$\mathrm{b} / \AA$ & $15.7942(4)$ \\
$\mathrm{c} / \AA$ & $17.7577(5)$ \\
$\alpha /{ }^{\circ}$ & 90 \\
$\beta /{ }^{\circ}$ & $100.851(3)$ \\
\hline
\end{tabular}




\begin{tabular}{ll}
\hline$\gamma /{ }^{\circ}$ & 90 \\
Volume $/ \AA^{3}$ & $3734.07(18)$ \\
$\mathrm{Z}$ & 4 \\
$\rho_{\text {calc }} / \mathrm{cm}^{3}$ & 1.554 \\
$\mu / \mathrm{mm}^{-1}$ & 0.803 \\
$\mathrm{~F}(000)$ & 1796.0 \\
Crystal size $/ \mathrm{mm}^{3}$ & $0.26 \times 0.21 \times 0.15$ \\
Radiation & $\mathrm{MoK} \alpha(\lambda=0.71073)$ \\
$2 \Theta$ range for data collection $/{ }^{\circ}$ & 3.478 to 62.106 \\
Index ranges & $-17 \leq \mathrm{h} \leq 18,-22 \leq \mathrm{k} \leq 20,-24 \leq 1 \leq 21$ \\
Reflections collected & 33417 \\
Independent reflections & $9646\left[\mathrm{R}_{\text {int }}=0.0690, \mathrm{R}_{\text {sigma }}=0.0561\right]$ \\
Data/restraints $/$ parameters & $9646 / 0 / 506$ \\
Goodness-of-fit on $\mathrm{F}^{2}$ & 1.070 \\
Final R indexes $[\mathrm{I}>=2 \sigma(\mathrm{I})]$ & $\mathrm{R}_{1}=0.0915, \mathrm{wR}_{2}=0.2009$ \\
Final R indexes $[$ all data $]$ & $\mathrm{R}_{1}=0.1376, \mathrm{wR}_{2}=0.2285$ \\
Largest diff. peak $/$ hole $/ \mathrm{e} \AA^{-3} 1.50 /-0.52$ \\
\hline \multicolumn{2}{c}{${ }^{\mathrm{a}} R_{1}=\Sigma|| F_{\mathrm{o}}|-| F_{\mathrm{c}}|| / \Sigma\left|F_{\mathrm{o}}\right| ;{ }^{\mathrm{b}} w R_{2}=\left[\Sigma w\left(F_{\mathrm{o}}{ }^{2}-F_{\mathrm{c}}{ }^{2}\right)^{2} / \Sigma w\left(F_{\mathrm{o}}^{2}\right)^{2}\right]^{1 / 2}}$. \\
\end{tabular}

Table S14. Selected bond lengths ( $\AA$ ) for $\mathbf{C o 5}$.

\begin{tabular}{|c|c|c|c|c|c|}
\hline \multicolumn{2}{|c|}{ Atom Atom } & \multirow{2}{*}{$\frac{\text { Length/Å }}{2.001(3)}$} & \multicolumn{2}{|c|}{ Atom Atom } & \multirow{2}{*}{$\frac{\text { Length/Å }}{1.476(6)}$} \\
\hline $\mathrm{Col}$ & $\mathrm{O} 1$ & & $\mathrm{C} 12$ & $\mathrm{C} 11$ & \\
\hline Col & $\mathrm{O} 2$ & $2.033(3)$ & $\mathrm{C} 12$ & $\mathrm{C} 13$ & $1.416(6)$ \\
\hline Col & $\mathrm{N} 3$ & $2.148(4)$ & $\mathrm{C} 7$ & C6 & $1.455(6)$ \\
\hline Col & N1 & $2.226(4)$ & $\mathrm{C} 7$ & $\mathrm{C} 8$ & $1.390(6)$ \\
\hline Col & $\mathrm{N} 2$ & $2.198(4)$ & $\mathrm{C} 3$ & $\mathrm{C} 2$ & $1.414(6)$ \\
\hline Col & N4 & $2.145(4)$ & C3 & $\mathrm{C} 4$ & $1.366(7)$ \\
\hline $\mathrm{Cl} 3$ & $\mathrm{C} 18$ & $1.748(5)$ & $\mathrm{C} 25$ & $\mathrm{C} 24$ & $1.403(6)$ \\
\hline $\mathrm{Cl} 2$ & $\mathrm{C} 8$ & $1.744(4)$ & $\mathrm{C} 25$ & $\mathrm{C} 26$ & $1.451(6)$ \\
\hline Cl1 & $\mathrm{C} 10$ & $1.751(5)$ & O6 & $\mathrm{C} 38$ & $1.416(7)$ \\
\hline $\mathrm{Cl} 4$ & $\mathrm{C} 20$ & $1.747(5)$ & $\mathrm{C} 17$ & $\mathrm{C} 18$ & $1.387(6)$ \\
\hline $\mathrm{O} 1$ & $\mathrm{C} 7$ & $1.288(5)$ & $\mathrm{C} 17$ & $\mathrm{C} 16$ & $1.448(6)$ \\
\hline $\mathrm{O} 2$ & $\mathrm{C} 17$ & $1.294(5)$ & $\mathrm{C} 32$ & $\mathrm{C} 31$ & $1.401(7)$ \\
\hline N3 & $\mathrm{C} 25$ & $1.359(5)$ & $\mathrm{C} 32$ & N4 & $1.331(6)$ \\
\hline N3 & $\mathrm{C} 21$ & $1.327(6)$ & $\mathrm{C} 14$ & $\mathrm{C} 15$ & $1.419(7)$ \\
\hline N1 & $\mathrm{C} 2$ & $1.323(5)$ & $\mathrm{C} 14$ & $\mathrm{C} 13$ & $1.356(7)$ \\
\hline N1 & C6 & $1.379(6)$ & $\mathrm{C} 27$ & $\mathrm{C} 26$ & $1.396(6)$ \\
\hline $\mathrm{O} 3$ & $\mathrm{C} 35$ & $1.411(7)$ & $\mathrm{C} 27$ & $\mathrm{C} 28$ & $1.465(6)$ \\
\hline $\mathrm{N} 2$ & $\mathrm{C} 12$ & $1.335(6)$ & $\mathrm{C} 27$ & $\mathrm{C} 30$ & $1.395(7)$ \\
\hline N2 & $\mathrm{C} 16$ & $1.376(6)$ & $\mathrm{C} 24$ & $\mathrm{C} 29$ & $1.458(6)$ \\
\hline O5 & $\mathrm{C} 37$ & $1.395(7)$ & $\mathrm{C} 21$ & $\mathrm{C} 22$ & $1.392(6)$ \\
\hline N5 & $\mathrm{C} 28$ & $1.358(6)$ & $\mathrm{C} 5$ & C6 & $1.408(6)$ \\
\hline N5 & C33 & $1.322(6)$ & $\mathrm{C} 5$ & $\mathrm{C} 4$ & $1.411(6)$ \\
\hline
\end{tabular}




\begin{tabular}{cccccc}
\hline $\mathrm{N} 6$ & $\mathrm{C} 29$ & $1.361(6)$ & $\mathrm{C} 26$ & $\mathrm{~N} 4$ & $1.347(5)$ \\
$\mathrm{N} 6$ & $\mathrm{C} 34$ & $1.329(6)$ & $\mathrm{C} 28$ & $\mathrm{C} 29$ & $1.387(6)$ \\
$\mathrm{O} 4$ & $\mathrm{C} 36$ & $1.345(8)$ & $\mathrm{C} 9$ & $\mathrm{C} 8$ & $1.396(6)$ \\
$\mathrm{C} 20$ & $\mathrm{C} 15$ & $1.413(7)$ & $\mathrm{C} 2$ & $\mathrm{C} 1$ & $1.489(6)$ \\
$\mathrm{C} 20$ & $\mathrm{C} 19$ & $1.365(7)$ & $\mathrm{C} 30$ & $\mathrm{C} 31$ & $1.371(7)$ \\
$\mathrm{C} 10$ & $\mathrm{C} 5$ & $1.413(6)$ & $\mathrm{C} 18$ & $\mathrm{C} 19$ & $1.396(7)$ \\
$\mathrm{C} 10$ & $\mathrm{C} 9$ & $1.360(7)$ & $\mathrm{C} 16$ & $\mathrm{C} 15$ & $1.411(6)$ \\
$\mathrm{C} 23$ & $\mathrm{C} 24$ & $1.399(6)$ & $\mathrm{C} 34$ & $\mathrm{C} 33$ & $1.391(8)$ \\
$\mathrm{C} 23$ & $\mathrm{C} 22$ & $1.372(7)$ & & & \\
\hline
\end{tabular}

Table S15. Selected bond angles $\left(^{\circ}\right)$ for $\mathbf{C o 5}$.

\begin{tabular}{|c|c|c|c|c|c|c|c|}
\hline \multicolumn{3}{|c|}{ Atom Atom Atom } & \multirow{2}{*}{$\frac{\text { Angle }^{\circ}}{178.05(12)}$} & \multicolumn{3}{|c|}{ Atom Atom Atom } & \multirow{2}{*}{$\frac{\text { Angle } /^{\circ}}{118.9(4)}$} \\
\hline $\mathrm{O} 1$ & Col & $\mathrm{O} 2$ & & $\mathrm{C} 26$ & $\mathrm{C} 27$ & $\mathrm{C} 28$ & \\
\hline $\mathrm{O} 1$ & Col & $\mathrm{N} 3$ & $87.97(13)$ & $\mathrm{C} 30$ & $\mathrm{C} 27$ & $\mathrm{C} 26$ & $118.4(4)$ \\
\hline O1 & Col & N1 & $79.11(12)$ & $\mathrm{C} 30$ & $\mathrm{C} 27$ & $\mathrm{C} 28$ & $122.7(4)$ \\
\hline $\mathrm{O} 1$ & Col & $\mathrm{N} 2$ & $101.81(13)$ & $\mathrm{C} 23$ & $\mathrm{C} 24$ & $\mathrm{C} 25$ & $117.5(4)$ \\
\hline $\mathrm{O} 1$ & Col & N4 & $91.27(12)$ & $\mathrm{C} 23$ & $\mathrm{C} 24$ & $\mathrm{C} 29$ & $123.9(4)$ \\
\hline $\mathrm{O} 2$ & Col & N3 & $90.08(13)$ & $\mathrm{C} 25$ & $\mathrm{C} 24$ & $\mathrm{C} 29$ & $118.5(4)$ \\
\hline $\mathrm{O} 2$ & Col & N1 & $102.82(13)$ & N3 & $\mathrm{C} 21$ & $\mathrm{C} 22$ & $123.5(4)$ \\
\hline $\mathrm{O} 2$ & Col & $\mathrm{N} 2$ & $78.47(13)$ & C6 & $\mathrm{C} 5$ & $\mathrm{C} 10$ & $118.1(4)$ \\
\hline $\mathrm{O} 2$ & Col & N4 & $88.28(13)$ & C6 & $\mathrm{C} 5$ & $\mathrm{C} 4$ & $117.4(4)$ \\
\hline N3 & Col & N1 & $165.09(14)$ & $\mathrm{C} 4$ & $\mathrm{C} 5$ & $\mathrm{C} 10$ & $124.5(4)$ \\
\hline N3 & Col & $\mathrm{N} 2$ & $97.78(14)$ & $\mathrm{C} 27$ & $\mathrm{C} 26$ & $\mathrm{C} 25$ & $120.4(4)$ \\
\hline $\mathrm{N} 2$ & Col & N1 & $92.17(13)$ & N4 & $\mathrm{C} 26$ & $\mathrm{C} 25$ & $117.4(4)$ \\
\hline N4 & Col & N3 & $77.06(13)$ & N4 & $\mathrm{C} 26$ & $\mathrm{C} 27$ & $122.2(4)$ \\
\hline N4 & Col & N1 & $95.66(13)$ & N5 & $\mathrm{C} 28$ & $\mathrm{C} 27$ & $117.1(4)$ \\
\hline N4 & Col & N2 & $165.84(14)$ & N5 & $\mathrm{C} 28$ & $\mathrm{C} 29$ & $122.4(4)$ \\
\hline $\mathrm{C} 7$ & $\mathrm{O} 1$ & Col & $116.7(3)$ & $\mathrm{C} 29$ & $\mathrm{C} 28$ & $\mathrm{C} 27$ & $120.4(4)$ \\
\hline $\mathrm{C} 17$ & $\mathrm{O} 2$ & Col & $115.6(3)$ & $\mathrm{C} 10$ & C9 & $\mathrm{C} 8$ & $119.1(4)$ \\
\hline $\mathrm{C} 25$ & N3 & Col & $114.6(3)$ & N1 & $\mathrm{C} 2$ & $\mathrm{C} 3$ & $121.9(4)$ \\
\hline $\mathrm{C} 21$ & N3 & $\mathrm{Col}$ & $127.7(3)$ & N1 & $\mathrm{C} 2$ & $\mathrm{C} 1$ & $119.7(4)$ \\
\hline $\mathrm{C} 21$ & N3 & $\mathrm{C} 25$ & $117.7(4)$ & $\mathrm{C} 3$ & $\mathrm{C} 2$ & $\mathrm{C} 1$ & $118.5(4)$ \\
\hline $\mathrm{C} 2$ & N1 & Col & $133.2(3)$ & N1 & C6 & $\mathrm{C} 7$ & $115.5(4)$ \\
\hline $\mathrm{C} 2$ & N1 & C6 & $118.4(4)$ & N1 & C6 & $\mathrm{C} 5$ & $122.7(4)$ \\
\hline C6 & $\mathrm{N} 1$ & Col & $108.4(3)$ & $\mathrm{C} 5$ & C6 & $\mathrm{C} 7$ & $121.8(4)$ \\
\hline $\mathrm{C} 12$ & $\mathrm{~N} 2$ & Col & 131.1(3) & N6 & $\mathrm{C} 29$ & $\mathrm{C} 24$ & $118.1(4)$ \\
\hline $\mathrm{C} 12$ & $\mathrm{~N} 2$ & $\mathrm{C} 16$ & $119.0(4)$ & N6 & $\mathrm{C} 29$ & $\mathrm{C} 28$ & $121.1(4)$ \\
\hline C16 & $\mathrm{N} 2$ & Col & $109.6(3)$ & $\mathrm{C} 28$ & $\mathrm{C} 29$ & $\mathrm{C} 24$ & $120.8(4)$ \\
\hline $\mathrm{C} 33$ & N5 & C28 & $115.4(4)$ & C31 & C30 & $\mathrm{C} 27$ & $119.2(4)$ \\
\hline $\mathrm{C} 34$ & N6 & $\mathrm{C} 29$ & $115.9(4)$ & $\mathrm{C} 17$ & $\mathrm{C} 18$ & $\mathrm{Cl} 3$ & $117.7(4)$ \\
\hline $\mathrm{C} 15$ & $\mathrm{C} 20$ & $\mathrm{Cl} 4$ & $119.5(4)$ & $\mathrm{C} 17$ & $\mathrm{C} 18$ & C19 & $124.5(4)$ \\
\hline $\mathrm{C} 19$ & C20 & $\mathrm{Cl} 4$ & $119.1(4)$ & C19 & C18 & $\mathrm{Cl} 3$ & $117.8(4)$ \\
\hline
\end{tabular}




\begin{tabular}{cccccccc}
\hline $\mathrm{C} 19$ & $\mathrm{C} 20$ & $\mathrm{C} 15$ & $121.3(4)$ & $\mathrm{N} 2$ & $\mathrm{C} 16$ & $\mathrm{C} 17$ & $115.2(4)$ \\
$\mathrm{C} 5$ & $\mathrm{C} 10$ & $\mathrm{C} 11$ & $119.2(4)$ & $\mathrm{N} 2$ & $\mathrm{C} 16$ & $\mathrm{C} 15$ & $123.2(4)$ \\
$\mathrm{C} 9$ & $\mathrm{C} 10$ & $\mathrm{C} 11$ & $118.9(4)$ & $\mathrm{C} 15$ & $\mathrm{C} 16$ & $\mathrm{C} 17$ & $121.6(4)$ \\
$\mathrm{C} 9$ & $\mathrm{C} 10$ & $\mathrm{C} 5$ & $121.8(4)$ & $\mathrm{C} 20$ & $\mathrm{C} 15$ & $\mathrm{C} 14$ & $125.2(4)$ \\
$\mathrm{C} 22$ & $\mathrm{C} 23$ & $\mathrm{C} 24$ & $119.7(4)$ & $\mathrm{C} 16$ & $\mathrm{C} 15$ & $\mathrm{C} 20$ & $118.5(4)$ \\
$\mathrm{N} 2$ & $\mathrm{C} 12$ & $\mathrm{C} 11$ & $119.3(4)$ & $\mathrm{C} 16$ & $\mathrm{C} 15$ & $\mathrm{C} 14$ & $116.3(4)$ \\
$\mathrm{N} 2$ & $\mathrm{C} 12$ & $\mathrm{C} 13$ & $120.3(4)$ & $\mathrm{C} 7$ & $\mathrm{C} 8$ & $\mathrm{C} 12$ & $117.2(3)$ \\
$\mathrm{C} 13$ & $\mathrm{C} 12$ & $\mathrm{C} 11$ & $120.4(4)$ & $\mathrm{C} 7$ & $\mathrm{C} 8$ & $\mathrm{C} 9$ & $124.3(4)$ \\
$\mathrm{O} 1$ & $\mathrm{C} 7$ & $\mathrm{C} 6$ & $120.1(4)$ & $\mathrm{C} 9$ & $\mathrm{C} 8$ & $\mathrm{C} 12$ & $118.6(3)$ \\
$\mathrm{O} 1$ & $\mathrm{C} 7$ & $\mathrm{C} 8$ & $125.0(4)$ & $\mathrm{C} 30$ & $\mathrm{C} 31$ & $\mathrm{C} 32$ & $119.2(4)$ \\
$\mathrm{C} 8$ & $\mathrm{C} 7$ & $\mathrm{C} 6$ & $114.9(4)$ & $\mathrm{C} 20$ & $\mathrm{C} 19$ & $\mathrm{C} 18$ & $119.0(4)$ \\
$\mathrm{C} 4$ & $\mathrm{C} 3$ & $\mathrm{C} 2$ & $120.4(4)$ & $\mathrm{C} 3$ & $\mathrm{C} 4$ & $\mathrm{C} 5$ & $119.2(4)$ \\
$\mathrm{N} 3$ & $\mathrm{C} 25$ & $\mathrm{C} 24$ & $122.8(4)$ & $\mathrm{C} 23$ & $\mathrm{C} 22$ & $\mathrm{C} 21$ & $118.7(4)$ \\
$\mathrm{N} 3$ & $\mathrm{C} 25$ & $\mathrm{C} 26$ & $116.4(4)$ & $\mathrm{C} 14$ & $\mathrm{C} 13$ & $\mathrm{C} 12$ & $121.4(4)$ \\
$\mathrm{C} 24$ & $\mathrm{C} 25$ & $\mathrm{C} 26$ & $120.8(4)$ & $\mathrm{N} 6$ & $\mathrm{C} 34$ & $\mathrm{C} 33$ & $122.3(4)$ \\
$\mathrm{O} 2$ & $\mathrm{C} 17$ & $\mathrm{C} 18$ & $125.2(4)$ & $\mathrm{N} 5$ & $\mathrm{C} 33$ & $\mathrm{C} 34$ & $122.8(4)$ \\
$\mathrm{O} 2$ & $\mathrm{C} 17$ & $\mathrm{C} 16$ & $119.7(4)$ & $\mathrm{C} 32$ & $\mathrm{~N} 4$ & $\mathrm{C} 1$ & $126.5(3)$ \\
$\mathrm{C} 18$ & $\mathrm{C} 17$ & $\mathrm{C} 16$ & $115.1(4)$ & $\mathrm{C} 32$ & $\mathrm{~N} 4$ & $\mathrm{C} 26$ & $118.9(4)$ \\
$\mathrm{N} 4$ & $\mathrm{C} 32$ & $\mathrm{C} 31$ & $122.2(4)$ & $\mathrm{C} 26$ & $\mathrm{~N} 4$ & $\mathrm{C} 1$ & $114.6(3)$ \\
$\mathrm{C} 13$ & $\mathrm{C} 14$ & $\mathrm{C} 15$ & $119.7(4)$ & & & & \\
\hline & & & & & & &
\end{tabular}

Table S16. Crystal data and structure refinement details for Co6.

\begin{tabular}{ll}
\hline Empirical formula & $\mathrm{C}_{35} \mathrm{H}_{24} \mathrm{Br}_{4} \mathrm{CoN}_{6} \mathrm{O}_{3}$ \\
Formula weight & 955.17 \\
Temperature/K & $100.00(10)$ \\
Crystal system & triclinic \\
Space group & $\mathrm{P}-1$ \\
$\mathrm{a} / \AA$ & $10.2172(4)$ \\
$\mathrm{b} / \AA$ & $10.8139(6)$ \\
$\mathrm{c} / \AA$ & $15.3313(8)$ \\
$\alpha /{ }^{\circ}$ & $85.108(4)$ \\
$\beta /{ }^{\circ}$ & $84.685(4)$ \\
$\gamma /{ }^{\circ}$ & $78.593(4)$ \\
Volume/ $\AA^{3}$ & $1649.43(14)$ \\
$\mathrm{Z}$ & 2 \\
$\rho_{\text {calc }} / \mathrm{cm}^{3}$ & 1.923 \\
$\mu / \mathrm{mm}^{-1}$ & 5.413 \\
$\mathrm{~F}(000)$ & 934.0 \\
Crystal size $/ \mathrm{mm}^{3}$ & $0.26 \times 0.21 \times 0.14$ \\
Radiation & $\mathrm{MoK} \alpha(\lambda=0.71073)$ \\
$2 \Theta$ range for data collection/ & 3.852 to 61.614 \\
Index ranges & $-14 \leq \mathrm{h} \leq 12,-14 \leq \mathrm{k} \leq 15,-22 \leq 1 \leq 21$ \\
\hline
\end{tabular}




\begin{tabular}{|c|c|}
\hline Reflections collected & 18919 \\
\hline Independent reflections & $8116\left[\mathrm{R}_{\text {int }}=0.0703, \mathrm{R}_{\text {sigma }}=0.0905\right]$ \\
\hline Data/restraints/parameters & $8116 / 0 / 446$ \\
\hline Goodness-of-fit on $\mathrm{F}^{2}$ & 1.087 \\
\hline Final $R$ indexes $[\mathrm{I}>=2 \sigma(\mathrm{I})]$ & $\mathrm{R}_{1}=0.0678, \mathrm{wR}_{2}=0.1878$ \\
\hline Final $\mathrm{R}$ indexes [all data] & $\mathrm{R}_{1}=0.1194, \mathrm{wR}_{2}=0.2142$ \\
\hline Largest diff. peak/hole / e $\AA$ & $1.73 /-1.24$ \\
\hline
\end{tabular}

Table S17. Selected bond lengths ( $\AA$ ) for Co6.

\begin{tabular}{|c|c|c|c|c|c|}
\hline \multicolumn{2}{|c|}{ Atom Atom } & \multirow{2}{*}{$\frac{\text { Length/Å }}{1.895(7)}$} & \multicolumn{2}{|c|}{ Atom Atom } & \multirow{2}{*}{$\frac{\text { Length/Å }}{1.454(9)}$} \\
\hline $\mathrm{Br} 2$ & $\mathrm{C} 22$ & & C9 & $\mathrm{C} 10$ & \\
\hline Br1 & $\mathrm{C} 24$ & $1.914(7)$ & C9 & C5 & $1.399(9)$ \\
\hline $\mathrm{Br} 3$ & $\mathrm{C} 32$ & $1.912(8)$ & C34 & $\mathrm{C} 29$ & $1.407(11)$ \\
\hline $\mathrm{Br} 4$ & $\mathrm{C} 34$ & $1.915(7)$ & C34 & C33 & $1.350(12)$ \\
\hline Col & $\mathrm{O} 2$ & $1.997(5)$ & $\mathrm{C} 30$ & C31 & $1.435(10)$ \\
\hline Col & $\mathrm{O} 1$ & $2.022(4)$ & $\mathrm{C} 30$ & C29 & $1.420(10)$ \\
\hline $\mathrm{Col}$ & N5 & $2.239(5)$ & $\mathrm{C} 3$ & $\mathrm{C} 11$ & $1.456(9)$ \\
\hline Col & N4 & $2.146(5)$ & $\mathrm{C} 3$ & $\mathrm{C} 4$ & $1.407(10)$ \\
\hline Col & N3 & $2.142(5)$ & C11 & $\mathrm{C} 10$ & $1.396(9)$ \\
\hline Col & N6 & $2.232(6)$ & $\mathrm{C} 11$ & C12 & $1.395(10)$ \\
\hline $\mathrm{O} 2$ & $\mathrm{C} 31$ & $1.289(8)$ & C6 & $\mathrm{C} 5$ & $1.406(10)$ \\
\hline $\mathrm{O} 1$ & $\mathrm{C} 21$ & $1.303(7)$ & C6 & $\mathrm{C} 7$ & $1.360(10)$ \\
\hline N5 & $\mathrm{C} 16$ & $1.331(9)$ & $\mathrm{C} 4$ & $\mathrm{C} 5$ & $1.447(9)$ \\
\hline N5 & $\mathrm{C} 20$ & $1.364(8)$ & C14 & C13 & $1.387(10)$ \\
\hline N4 & $\mathrm{C} 10$ & $1.352(8)$ & $\mathrm{C} 2$ & $\mathrm{C} 1$ & $1.399(11)$ \\
\hline N4 & $\mathrm{C} 14$ & $1.328(9)$ & $\mathrm{C} 28$ & $\mathrm{C} 29$ & $1.419(11)$ \\
\hline N3 & $\mathrm{C} 8$ & $1.328(9)$ & $\mathrm{C} 28$ & $\mathrm{C} 27$ & $1.356(12)$ \\
\hline N3 & C9 & $1.355(8)$ & $\mathrm{C} 12$ & $\mathrm{C} 13$ & $1.380(9)$ \\
\hline N6 & $\mathrm{C} 30$ & $1.375(9)$ & $\mathrm{C} 25$ & $\mathrm{C} 26$ & $1.489(10)$ \\
\hline N6 & $\mathrm{C} 26$ & $1.319(9)$ & $\mathrm{C} 32$ & C31 & $1.385(10)$ \\
\hline N2 & $\mathrm{C} 3$ & $1.358(8)$ & C32 & C33 & $1.388(11)$ \\
\hline N2 & $\mathrm{C} 2$ & $1.338(9)$ & $\mathrm{C} 17$ & C16 & $1.418(9)$ \\
\hline N1 & $\mathrm{C} 4$ & $1.355(8)$ & C19 & $\mathrm{C} 24$ & $1.413(10)$ \\
\hline N1 & $\mathrm{C} 1$ & $1.335(9)$ & C19 & $\mathrm{C} 20$ & $1.425(9)$ \\
\hline $\mathrm{C} 8$ & $\mathrm{C} 7$ & $1.406(10)$ & $\mathrm{C} 24$ & $\mathrm{C} 23$ & $1.358(11)$ \\
\hline $\mathrm{C} 21$ & $\mathrm{C} 20$ & $1.447(10)$ & $\mathrm{C} 23$ & $\mathrm{C} 22$ & $1.397(10)$ \\
\hline $\mathrm{C} 21$ & $\mathrm{C} 22$ & $1.395(9)$ & $\mathrm{C} 16$ & C15 & $1.487(9)$ \\
\hline $\mathrm{C} 18$ & $\mathrm{C} 17$ & $1.353(10)$ & $\mathrm{C} 27$ & $\mathrm{C} 26$ & $1.431(10)$ \\
\hline $\mathrm{C} 18$ & $\mathrm{C} 19$ & $1.414(10)$ & $\mathrm{O} 3$ & C35 & $1.417(16)$ \\
\hline
\end{tabular}


Table S18. Selected bond angles $\left(^{\circ}\right)$ for Co6.

\begin{tabular}{|c|c|c|c|c|c|c|c|}
\hline \multicolumn{3}{|c|}{ Atom Atom Atom } & \multirow{2}{*}{$\frac{\text { Angle }^{\circ}}{177.21(18)}$} & \multicolumn{3}{|c|}{ Atom Atom Atom } & \multirow{2}{*}{$\frac{\text { Angle }^{\circ}}{118.2(6)}$} \\
\hline $\mathrm{O} 2$ & Co1 & $\mathrm{O} 1$ & & $\mathrm{C} 12$ & $\mathrm{C} 11$ & $\mathrm{C} 10$ & \\
\hline $\mathrm{O} 2$ & Co1 & N5 & $101.3(2)$ & $\mathrm{C} 7$ & C6 & $\mathrm{C} 5$ & $119.9(6)$ \\
\hline $\mathrm{O} 2$ & Col & N4 & $92.29(19)$ & N4 & $\mathrm{C} 10$ & C9 & $116.6(6)$ \\
\hline $\mathrm{O} 2$ & Col & N3 & $91.5(2)$ & N4 & $\mathrm{C} 10$ & $\mathrm{C} 11$ & $123.1(6)$ \\
\hline $\mathrm{O} 2$ & Co1 & N6 & $78.05(19)$ & C11 & $\mathrm{C} 10$ & C9 & $120.3(6)$ \\
\hline $\mathrm{O} 1$ & Col & N5 & $78.06(19)$ & N1 & $\mathrm{C} 4$ & $\mathrm{C} 3$ & $121.6(6)$ \\
\hline $\mathrm{O} 1$ & Col & N4 & $88.72(19)$ & N1 & $\mathrm{C} 4$ & $\mathrm{C} 5$ & $117.9(6)$ \\
\hline $\mathrm{O} 1$ & Co1 & N3 & $91.27(19)$ & $\mathrm{C} 3$ & $\mathrm{C} 4$ & $\mathrm{C} 5$ & $120.5(6)$ \\
\hline $\mathrm{O} 1$ & Col & N6 & 99.21(19) & N4 & $\mathrm{C} 14$ & $\mathrm{C} 13$ & $123.5(6)$ \\
\hline N4 & Col & N5 & $164.8(2)$ & $\mathrm{N} 2$ & $\mathrm{C} 2$ & $\mathrm{C} 1$ & $123.3(6)$ \\
\hline N4 & Co1 & N6 & $100.5(2)$ & $\mathrm{C} 27$ & $\mathrm{C} 28$ & $\mathrm{C} 29$ & $120.4(7)$ \\
\hline N3 & Col & N5 & $95.4(2)$ & $\mathrm{C} 13$ & $\mathrm{C} 12$ & $\mathrm{C} 11$ & $118.5(6)$ \\
\hline N3 & Col & N4 & $77.2(2)$ & C31 & C32 & $\mathrm{Br} 3$ & $117.7(5)$ \\
\hline N3 & Col & N6 & $169.2(2)$ & C31 & C32 & C33 & $124.4(7)$ \\
\hline N6 & Col & N5 & $89.20(19)$ & C33 & C32 & $\mathrm{Br} 3$ & $117.9(6)$ \\
\hline $\mathrm{C} 31$ & $\mathrm{O} 2$ & Co1 & $115.4(4)$ & $\mathrm{C} 12$ & C13 & $\mathrm{C} 14$ & $119.3(7)$ \\
\hline $\mathrm{C} 21$ & $\mathrm{O} 1$ & Co1 & $115.3(4)$ & N1 & $\mathrm{C} 1$ & $\mathrm{C} 2$ & $122.0(6)$ \\
\hline $\mathrm{C} 16$ & N5 & Col & $132.1(4)$ & $\mathrm{O} 2$ & C31 & $\mathrm{C} 30$ & $120.1(6)$ \\
\hline $\mathrm{C} 16$ & N5 & $\mathrm{C} 20$ & $119.4(6)$ & $\mathrm{O} 2$ & C31 & C32 & $125.3(6)$ \\
\hline $\mathrm{C} 20$ & N5 & Col & $108.2(4)$ & C32 & C31 & $\mathrm{C} 30$ & $114.6(6)$ \\
\hline $\mathrm{C} 10$ & N4 & Col & $114.7(4)$ & C18 & $\mathrm{C} 17$ & $\mathrm{C} 16$ & $121.7(7)$ \\
\hline $\mathrm{C} 14$ & N4 & Co1 & $128.0(5)$ & C9 & $\mathrm{C} 5$ & C6 & $117.4(6)$ \\
\hline $\mathrm{C} 14$ & N4 & $\mathrm{C} 10$ & $117.4(6)$ & $\mathrm{C} 9$ & $\mathrm{C} 5$ & $\mathrm{C} 4$ & $119.4(6)$ \\
\hline $\mathrm{C} 8$ & N3 & Co1 & $126.9(4)$ & C6 & $\mathrm{C} 5$ & $\mathrm{C} 4$ & $123.2(6)$ \\
\hline $\mathrm{C} 8$ & N3 & C9 & $118.6(6)$ & C18 & C19 & $\mathrm{C} 20$ & $117.0(6)$ \\
\hline C9 & N3 & Col & $114.4(4)$ & C24 & C19 & $\mathrm{C} 18$ & $125.3(6)$ \\
\hline $\mathrm{C} 30$ & N6 & Col & $107.1(4)$ & C24 & C19 & $\mathrm{C} 20$ & $117.7(7)$ \\
\hline $\mathrm{C} 26$ & N6 & Co1 & $133.2(5)$ & C19 & $\mathrm{C} 24$ & Br1 & $119.6(6)$ \\
\hline $\mathrm{C} 26$ & N6 & C30 & $119.1(6)$ & $\mathrm{C} 23$ & $\mathrm{C} 24$ & $\mathrm{Br} 1$ & $118.8(5)$ \\
\hline $\mathrm{C} 2$ & N2 & C3 & $114.8(6)$ & $\mathrm{C} 23$ & $\mathrm{C} 24$ & $\mathrm{C} 19$ & $121.6(6)$ \\
\hline $\mathrm{C} 1$ & N1 & $\mathrm{C} 4$ & $116.0(6)$ & C24 & $\mathrm{C} 23$ & $\mathrm{C} 22$ & $120.0(7)$ \\
\hline N3 & $\mathrm{C} 8$ & $\mathrm{C} 7$ & $122.4(6)$ & N5 & $\mathrm{C} 16$ & $\mathrm{C} 17$ & $120.3(6)$ \\
\hline $\mathrm{O} 1$ & $\mathrm{C} 21$ & $\mathrm{C} 20$ & $119.9(5)$ & N5 & $\mathrm{C} 16$ & $\mathrm{C} 15$ & $119.8(6)$ \\
\hline $\mathrm{O} 1$ & $\mathrm{C} 21$ & $\mathrm{C} 22$ & $124.7(6)$ & $\mathrm{C} 17$ & $\mathrm{C} 16$ & $\mathrm{C} 15$ & $119.9(6)$ \\
\hline $\mathrm{C} 22$ & $\mathrm{C} 21$ & $\mathrm{C} 20$ & $115.4(6)$ & N5 & $\mathrm{C} 20$ & $\mathrm{C} 21$ & $115.7(6)$ \\
\hline $\mathrm{C} 17$ & C18 & C19 & $119.0(6)$ & N5 & $\mathrm{C} 20$ & $\mathrm{C} 19$ & $122.4(6)$ \\
\hline N3 & C9 & $\mathrm{C} 10$ & $117.0(6)$ & C19 & $\mathrm{C} 20$ & $\mathrm{C} 21$ & $121.8(6)$ \\
\hline N3 & C9 & $\mathrm{C} 5$ & $122.6(6)$ & C34 & C29 & $\mathrm{C} 30$ & $117.2(7)$ \\
\hline $\mathrm{C} 5$ & C9 & $\mathrm{C} 10$ & $120.4(6)$ & C34 & C29 & $\mathrm{C} 28$ & $126.1(7)$ \\
\hline $\mathrm{C} 29$ & C34 & $\mathrm{Br} 4$ & $118.9(6)$ & C28 & C29 & $\mathrm{C} 30$ & $116.6(7)$ \\
\hline $\mathrm{C} 33$ & C34 & $\mathrm{Br} 4$ & $119.3(6)$ & C6 & $\mathrm{C} 7$ & $\mathrm{C} 8$ & $119.0(7)$ \\
\hline $\mathrm{C} 33$ & C34 & $\mathrm{C} 29$ & $121.8(7)$ & $\mathrm{C} 21$ & $\mathrm{C} 22$ & $\mathrm{Br} 2$ & $118.1(5)$ \\
\hline
\end{tabular}




\begin{tabular}{cccccccc}
\hline $\mathrm{N} 6$ & $\mathrm{C} 30$ & $\mathrm{C} 31$ & $115.2(6)$ & $\mathrm{C} 21$ & $\mathrm{C} 22$ & $\mathrm{C} 23$ & $123.4(7)$ \\
$\mathrm{N} 6$ & $\mathrm{C} 30$ & $\mathrm{C} 29$ & $122.4(7)$ & $\mathrm{C} 23$ & $\mathrm{C} 22$ & $\mathrm{Br} 2$ & $118.5(5)$ \\
$\mathrm{C} 29$ & $\mathrm{C} 30$ & $\mathrm{C} 31$ & $122.4(6)$ & $\mathrm{C} 28$ & $\mathrm{C} 27$ & $\mathrm{C} 26$ & $119.6(7)$ \\
$\mathrm{N} 2$ & $\mathrm{C} 3$ & $\mathrm{C} 11$ & $118.0(6)$ & $\mathrm{N} 6$ & $\mathrm{C} 26$ & $\mathrm{C} 25$ & $120.0(6)$ \\
$\mathrm{N} 2$ & $\mathrm{C} 3$ & $\mathrm{C} 4$ & $122.2(6)$ & $\mathrm{N} 6$ & $\mathrm{C} 26$ & $\mathrm{C} 27$ & $121.7(7)$ \\
$\mathrm{C} 4$ & $\mathrm{C} 3$ & $\mathrm{C} 11$ & $119.8(6)$ & $\mathrm{C} 27$ & $\mathrm{C} 26$ & $\mathrm{C} 25$ & $118.3(7)$ \\
$\mathrm{C} 10$ & $\mathrm{C} 11$ & $\mathrm{C} 3$ & $119.6(6)$ & $\mathrm{C} 34$ & $\mathrm{C} 33$ & $\mathrm{C} 32$ & $119.4(7)$ \\
$\mathrm{C} 12$ & $\mathrm{C} 11$ & $\mathrm{C} 3$ & $122.2(6)$ & & & & \\
\hline
\end{tabular}

Table S19. Crystal data and structure refinement details for Co7.

\begin{tabular}{|c|c|}
\hline Empirical formula & $\mathrm{C}_{39} \mathrm{H}_{26} \mathrm{Cl}_{4} \mathrm{CoN}_{6} \mathrm{O}_{3}$ \\
\hline Formula weight & 827.39 \\
\hline Temperature/K & $100.00(10)$ \\
\hline Crystal system & triclinic \\
\hline Space group & P-1 \\
\hline $\mathrm{a} / \AA$ & $11.0714(3)$ \\
\hline $\mathrm{b} / \AA$ & $11.9810(4)$ \\
\hline $\mathrm{c} / \AA$ & $14.1583(4)$ \\
\hline$\alpha /^{\circ}$ & $81.761(3)$ \\
\hline$\beta /{ }^{\circ}$ & $88.903(2)$ \\
\hline$\gamma /{ }^{\circ}$ & $84.720(3)$ \\
\hline Volume $/ \AA^{3}$ & $1850.74(10)$ \\
\hline Z & 2 \\
\hline$\rho_{\text {calc }} \mathrm{g} / \mathrm{cm}^{3}$ & 1.485 \\
\hline$\mu / \mathrm{mm}^{-1}$ & 0.800 \\
\hline $\mathrm{F}(000)$ & 842.0 \\
\hline Crystal size $/ \mathrm{mm}^{3}$ & $0.29 \times 0.21 \times 0.15$ \\
\hline Radiation & $\operatorname{MoK} \alpha(\lambda=0.71073)$ \\
\hline \multicolumn{2}{|c|}{$2 \Theta$ range for data collection $/{ }^{\circ} 4.182$ to 61.988} \\
\hline Index ranges & $-13 \leq \mathrm{h} \leq 14,-17 \leq \mathrm{k} \leq 16,-20 \leq 1 \leq 19$ \\
\hline Reflections collected & 29358 \\
\hline Independent reflections & $9340\left[\mathrm{R}_{\text {int }}=0.0366, \mathrm{R}_{\text {sigma }}=0.0413\right]$ \\
\hline Data/restraints/parameters & $9340 / 0 / 482$ \\
\hline Goodness-of-fit on $\mathrm{F}^{2}$ & 1.045 \\
\hline Final $\mathrm{R}$ indexes $[\mathrm{I}>=2 \sigma(\mathrm{I})]$ & $\mathrm{R}_{1}=0.0416, \mathrm{wR}_{2}=0.1071$ \\
\hline Final R indexes [all data] & $\mathrm{R}_{1}=0.0610, \mathrm{wR}_{2}=0.1176$ \\
\hline Largest diff. peak/hole / e $\AA$ & $0.56 /-0.42$ \\
\hline
\end{tabular}


Table S20. Selected bond lengths ( $\AA$ ) for Co7.

\begin{tabular}{cccccc}
\hline Atom & Atom & Length/Å & Atom Atom & Length/A \\
\hline Co1 & O2 & $2.0341(14)$ & C33 & C29 & $1.394(3)$ \\
Co1 & O1 & $2.0202(14)$ & C6 & C5 & $1.416(3)$ \\
Co1 & N5 & $2.1391(18)$ & C6 & C7 & $1.448(3)$ \\
Co1 & N6 & $2.1402(17)$ & C25 & C24 & $1.419(3)$ \\
Co1 & N1 & $2.2285(18)$ & C29 & C28 & $1.466(3)$ \\
Co1 & N2 & $2.2111(17)$ & C29 & C30 & $1.409(3)$ \\
C11 & C8 & $1.744(2)$ & C5 & C10 & $1.411(3)$ \\
C14 & C18 & $1.735(2)$ & C5 & C4 & $1.420(3)$ \\
C15 & C20 & $1.740(2)$ & C17 & C16 & $1.435(3)$ \\
C18 & C10 & $1.741(2)$ & C17 & C18 & $1.391(3)$ \\
O2 & C17 & $1.301(2)$ & C7 & C8 & $1.399(3)$ \\
O1 & C7 & $1.291(3)$ & C1 & C2 & $1.501(3)$ \\
N5 & C33 & $1.357(3)$ & C2 & C3 & $1.413(3)$ \\
N5 & C32 & $1.333(3)$ & C36 & C37 & $1.369(3)$ \\
N6 & C34 & $1.348(3)$ & C8 & C9 & $1.396(3)$ \\
N6 & C38 & $1.333(3)$ & C32 & C31 & $1.387(3)$ \\
N1 & C6 & $1.373(3)$ & C16 & C15 & $1.418(3)$ \\
N1 & C2 & $1.329(3)$ & C31 & C30 & $1.375(3)$ \\
O3 & C39 & $1.412(3)$ & C21 & C22 & $1.362(3)$ \\
N3 & C27 & $1.323(3)$ & C37 & C38 & $1.393(3)$ \\
N3 & C26 & $1.353(3)$ & C15 & C20 & $1.416(3)$ \\
N2 & C16 & $1.381(3)$ & C15 & C14 & $1.410(3)$ \\
N2 & C12 & $1.327(3)$ & C24 & C23 & $1.369(3)$ \\
N4 & C25 & $1.354(3)$ & C20 & C19 & $1.360(3)$ \\
N4 & C28 & $1.326(3)$ & C18 & C19 & $1.397(3)$ \\
C35 & C27 & $1.456(3)$ & C23 & C22 & $1.417(3)$ \\
C35 & C34 & $1.392(3)$ & C10 & C9 & $1.366(3)$ \\
C35 & C36 & $1.404(3)$ & C12 & C11 & $1.494(3)$ \\
C27 & C28 & $1.435(3)$ & C12 & C13 & $1.419(3)$ \\
C34 & C33 & $1.460(3)$ & C3 & C4 & $1.361(3)$ \\
C26 & C25 & $1.425(3)$ & C14 & C13 & $1.355(4)$ \\
C26 & C21 & $1.415(3)$ & & & \\
\hline
\end{tabular}

Table S21. Selected bond angles $\left(^{\circ}\right)$ for $\mathrm{Co} 7$.

\begin{tabular}{cccccccc}
\hline Atom Atom Atom & Angle $^{\circ}$ & \multicolumn{4}{c}{ Atom Atom Atom } & Angle ${ }^{\circ}$ \\
\hline O2 & Co1 & N5 & $90.82(6)$ & C30 & C29 & C28 & $122.19(19)$ \\
O2 & Co1 & N6 & $87.12(6)$ & C6 & C5 & C4 & $117.1(2)$ \\
\hline \multicolumn{5}{c}{ S19 }
\end{tabular}




\begin{tabular}{|c|c|c|c|c|c|c|c|}
\hline $\mathrm{O} 2$ & Col & N1 & $104.67(6)$ & $\mathrm{C} 10$ & $\mathrm{C} 5$ & C6 & $118.29(19)$ \\
\hline $\mathrm{O} 2$ & Col & $\mathrm{N} 2$ & $78.47(6)$ & $\mathrm{C} 10$ & $\mathrm{C} 5$ & $\mathrm{C} 4$ & $124.6(2)$ \\
\hline $\mathrm{O} 1$ & Col & $\mathrm{O} 2$ & $176.64(6)$ & $\mathrm{O} 2$ & $\mathrm{C} 17$ & $\mathrm{C} 16$ & $119.89(18)$ \\
\hline $\mathrm{O} 1$ & Col & N5 & $85.85(6)$ & $\mathrm{O} 2$ & $\mathrm{C} 17$ & C18 & $124.14(19)$ \\
\hline $\mathrm{O} 1$ & Col & N6 & $91.69(6)$ & $\mathrm{C} 18$ & $\mathrm{C} 17$ & $\mathrm{C} 16$ & $115.96(19)$ \\
\hline $\mathrm{O} 1$ & Col & N1 & $78.53(6)$ & $\mathrm{O} 1$ & $\mathrm{C} 7$ & C6 & $120.38(18)$ \\
\hline $\mathrm{O} 1$ & Col & $\mathrm{N} 2$ & $102.56(6)$ & $\mathrm{O} 1$ & $\mathrm{C} 7$ & $\mathrm{C} 8$ & $124.39(19)$ \\
\hline N5 & Col & N6 & $77.62(6)$ & $\mathrm{C} 8$ & $\mathrm{C} 7$ & C6 & $115.23(19)$ \\
\hline N5 & Col & N1 & $161.85(6)$ & N4 & $\mathrm{C} 28$ & $\mathrm{C} 27$ & $121.92(18)$ \\
\hline N5 & Co1 & $\mathrm{N} 2$ & $99.84(6)$ & N4 & $\mathrm{C} 28$ & C29 & $118.63(18)$ \\
\hline N6 & Col & N1 & $93.52(6)$ & $\mathrm{C} 27$ & $\mathrm{C} 28$ & C29 & $119.45(18)$ \\
\hline N6 & Col & $\mathrm{N} 2$ & $165.36(7)$ & N1 & $\mathrm{C} 2$ & $\mathrm{C} 1$ & $118.8(2)$ \\
\hline $\mathrm{N} 2$ & Col & N1 & $92.60(6)$ & N1 & $\mathrm{C} 2$ & $\mathrm{C} 3$ & $121.7(2)$ \\
\hline $\mathrm{C} 17$ & $\mathrm{O} 2$ & Co1 & $115.44(13)$ & $\mathrm{C} 3$ & $\mathrm{C} 2$ & $\mathrm{C} 1$ & $119.5(2)$ \\
\hline $\mathrm{C} 7$ & $\mathrm{O} 1$ & Col & $116.43(13)$ & C37 & $\mathrm{C} 36$ & C35 & $119.07(19)$ \\
\hline C33 & N5 & Co1 & $114.00(13)$ & $\mathrm{C} 7$ & $\mathrm{C} 8$ & Cl1 & $118.83(17)$ \\
\hline C32 & N5 & Col & $127.71(15)$ & C9 & $\mathrm{C} 8$ & $\mathrm{Cl1}$ & 117.61(16) \\
\hline $\mathrm{C} 32$ & N5 & C33 & $118.12(18)$ & C9 & $\mathrm{C} 8$ & $\mathrm{C} 7$ & $123.5(2)$ \\
\hline C34 & N6 & Co1 & $114.30(13)$ & N5 & $\mathrm{C} 32$ & C31 & $123.0(2)$ \\
\hline C38 & N6 & Co1 & $127.45(15)$ & N2 & $\mathrm{C} 16$ & $\mathrm{C} 17$ & $115.89(18)$ \\
\hline C38 & N6 & C34 & $118.24(18)$ & N2 & $\mathrm{C} 16$ & $\mathrm{C} 15$ & $122.28(19)$ \\
\hline C6 & N1 & Co1 & $109.11(13)$ & $\mathrm{C} 15$ & $\mathrm{C} 16$ & $\mathrm{C} 17$ & $121.83(19)$ \\
\hline $\mathrm{C} 2$ & N1 & Co1 & $132.18(15)$ & $\mathrm{C} 30$ & $\mathrm{C} 31$ & C32 & $119.49(19)$ \\
\hline $\mathrm{C} 2$ & N1 & C6 & $118.70(19)$ & $\mathrm{C} 22$ & $\mathrm{C} 21$ & C26 & $119.9(2)$ \\
\hline $\mathrm{C} 27$ & N3 & $\mathrm{C} 26$ & $116.95(18)$ & C31 & $\mathrm{C} 30$ & C29 & $118.64(19)$ \\
\hline $\mathrm{C} 16$ & $\mathrm{~N} 2$ & Col & $108.80(13)$ & C36 & $\mathrm{C} 37$ & C38 & 119.11(19) \\
\hline $\mathrm{C} 12$ & $\mathrm{~N} 2$ & Co1 & $132.11(15)$ & $\mathrm{C} 20$ & $\mathrm{C} 15$ & $\mathrm{C} 16$ & $117.9(2)$ \\
\hline $\mathrm{C} 12$ & $\mathrm{~N} 2$ & $\mathrm{C} 16$ & $118.74(18)$ & $\mathrm{C} 14$ & $\mathrm{C} 15$ & $\mathrm{C} 16$ & $117.3(2)$ \\
\hline $\mathrm{C} 28$ & $\mathrm{~N} 4$ & $\mathrm{C} 25$ & $116.72(18)$ & C14 & $\mathrm{C} 15$ & C20 & $124.8(2)$ \\
\hline C34 & C35 & $\mathrm{C} 27$ & 119.91(18) & $\mathrm{C} 23$ & $\mathrm{C} 24$ & $\mathrm{C} 25$ & $119.5(2)$ \\
\hline C34 & C35 & C36 & $118.15(19)$ & $\mathrm{C} 15$ & $\mathrm{C} 20$ & $\mathrm{C} 15$ & $118.86(18)$ \\
\hline C36 & C35 & $\mathrm{C} 27$ & $121.95(18)$ & C19 & $\mathrm{C} 20$ & $\mathrm{C} 15$ & $119.96(18)$ \\
\hline N3 & $\mathrm{C} 27$ & C35 & $118.42(18)$ & C19 & $\mathrm{C} 20$ & C15 & $121.2(2)$ \\
\hline N3 & $\mathrm{C} 27$ & $\mathrm{C} 28$ & $121.76(19)$ & $\mathrm{C} 17$ & $\mathrm{C} 18$ & $\mathrm{Cl} 4$ & $118.20(16)$ \\
\hline $\mathrm{C} 28$ & $\mathrm{C} 27$ & C35 & $119.81(18)$ & $\mathrm{C} 17$ & $\mathrm{C} 18$ & C19 & $123.1(2)$ \\
\hline N6 & C34 & C35 & $122.59(18)$ & C19 & $\mathrm{C} 18$ & $\mathrm{Cl} 4$ & $118.68(17)$ \\
\hline N6 & C34 & C33 & $116.89(17)$ & C24 & $\mathrm{C} 23$ & $\mathrm{C} 22$ & $121.1(2)$ \\
\hline $\mathrm{C} 35$ & C34 & C33 & $120.52(18)$ & N6 & $\mathrm{C} 38$ & C37 & $122.8(2)$ \\
\hline N3 & C26 & $\mathrm{C} 25$ & $121.34(19)$ & $\mathrm{C} 5$ & $\mathrm{C} 10$ & $\mathrm{Cl} 8$ & $119.85(18)$ \\
\hline N3 & $\mathrm{C} 26$ & $\mathrm{C} 21$ & $119.10(19)$ & C9 & $\mathrm{C} 10$ & $\mathrm{Cl} 8$ & $118.90(17)$ \\
\hline $\mathrm{C} 21$ & C26 & $\mathrm{C} 25$ & $119.56(19)$ & C9 & $\mathrm{C} 10$ & $\mathrm{C} 5$ & $121.2(2)$ \\
\hline N5 & C33 & C34 & $116.79(18)$ & $\mathrm{C} 21$ & $\mathrm{C} 22$ & $\mathrm{C} 23$ & $120.6(2)$ \\
\hline N5 & C33 & C29 & $122.48(18)$ & N2 & $\mathrm{C} 12$ & $\mathrm{C} 11$ & $119.8(2)$ \\
\hline $\mathrm{C} 29$ & C33 & C34 & $120.72(18)$ & N2 & $\mathrm{C} 12$ & $\mathrm{C} 13$ & $121.4(2)$ \\
\hline N1 & C6 & $\mathrm{C} 5$ & $122.61(19)$ & C13 & $\mathrm{C} 12$ & C11 & $118.8(2)$ \\
\hline
\end{tabular}




\begin{tabular}{cccccccc}
\hline N1 & C6 & C7 & $115.46(18)$ & C4 & C3 & C2 & $120.7(2)$ \\
C5 & C6 & C7 & $121.87(19)$ & C20 & C19 & C18 & $120.0(2)$ \\
N4 & C25 & C26 & $121.28(19)$ & C10 & C9 & C8 & $119.9(2)$ \\
N4 & C25 & C24 & $119.40(19)$ & C3 & C4 & C5 & $119.2(2)$ \\
C24 & C25 & C26 & $119.31(19)$ & C13 & C14 & C15 & $119.6(2)$ \\
C33 & C29 & C28 & $119.56(18)$ & C14 & C13 & C12 & $120.7(2)$ \\
C33 & C29 & C30 & $118.24(18)$ & & & & \\
\hline
\end{tabular}

Table S22. Crystal data and structure refinement details for $\mathbf{C o 8}$.

\begin{tabular}{|c|c|}
\hline Empirical formula & $\mathrm{C}_{34} \mathrm{H}_{29} \mathrm{Cl}_{4} \mathrm{CoN}_{4} \mathrm{O}_{5}$ \\
\hline Formula weight & 774.34 \\
\hline Temperature/K & $293(2)$ \\
\hline Crystal system & triclinic \\
\hline Space group & $\mathrm{P}-1$ \\
\hline $\mathrm{a} / \AA$ & $9.4751(4)$ \\
\hline $\mathrm{b} / \AA ̊$ & $12.9221(6)$ \\
\hline $\mathrm{c} / \AA ̊$ & $14.5515(5)$ \\
\hline$\alpha^{\circ}$ & $86.950(3)$ \\
\hline$\beta /{ }^{\circ}$ & $80.436(3)$ \\
\hline$\gamma /{ }^{\circ}$ & $75.903(4)$ \\
\hline Volume $/ \AA^{3}$ & $1703.84(12)$ \\
\hline $\mathrm{Z}$ & 2 \\
\hline$\rho_{\text {calc }} \mathrm{mg} / \mathrm{mm}^{3}$ & 1.509 \\
\hline $\mathrm{m} / \mathrm{mm}^{-1}$ & 0.865 \\
\hline $\mathrm{F}(000)$ & 792.0 \\
\hline Crystal size $/ \mathrm{mm}^{3}$ & $? \times ? \times ?$ \\
\hline $2 \Theta$ range for data collection & 6.66 to $52.746^{\circ}$ \\
\hline Index ranges & $-11 \leq \mathrm{h} \leq 11,-16 \leq \mathrm{k} \leq 16,-17 \leq 1 \leq 18$ \\
\hline Reflections collected & 12060 \\
\hline Independent reflections & $6942[\mathrm{R}(\mathrm{int})=0.0373]$ \\
\hline Data/restraints/parameters & $6942 / 1512 / 437$ \\
\hline Goodness-of-fit on $\mathrm{F}^{2}$ & 1.041 \\
\hline Final $R$ indexes $[\mathrm{I}>=2 \sigma(\mathrm{I})]$ & $\mathrm{R}_{1}=0.0937, \mathrm{wR}_{2}=0.2410$ \\
\hline Final $\mathrm{R}$ indexes [all data] & $\mathrm{R}_{1}=0.1549, \mathrm{wR}_{2}=0.2882$ \\
\hline Largest diff. peak/hole / e $\AA^{-3}$ & ${ }^{3} 1.20 /-1.80$ \\
\hline
\end{tabular}


Table S23. Selected bond lengths ( $\AA$ ) for Co8.

\begin{tabular}{|c|c|c|c|c|c|}
\hline \multicolumn{2}{|c|}{ Atom Atom } & \multirow{2}{*}{$\frac{\text { Length/§̊ }}{2.026(5)}$} & \multicolumn{2}{|c|}{ Atom Atom } & \multirow{2}{*}{$\frac{\text { Length/Å }}{1.517(12)}$} \\
\hline $\mathrm{Col}$ & $\mathrm{O} 1$ & & $\mathrm{C} 7$ & $\mathrm{C} 8$ & \\
\hline Col & $\mathrm{O} 2$ & $2.029(5)$ & $\mathrm{C} 7$ & C9 & $1.459(12)$ \\
\hline Col & N1 & $2.262(6)$ & C9 & $\mathrm{C} 10$ & $1.394(13)$ \\
\hline Col & N2 & $2.221(6)$ & C9 & C13 & $1.400(11)$ \\
\hline Col & N3 & $2.146(6)$ & $\mathrm{C} 10$ & $\mathrm{C} 11$ & $1.364(13)$ \\
\hline Col & N4 & $2.123(6)$ & $\mathrm{C} 11$ & $\mathrm{C} 12$ & $1.398(11)$ \\
\hline $\mathrm{Cl1}$ & $\mathrm{C} 30$ & $1.754(8)$ & $\mathrm{C} 13$ & C14 & $1.444(12)$ \\
\hline $\mathrm{Cl} 2$ & $\mathrm{C} 32$ & $1.739(7)$ & $\mathrm{C} 15$ & $\mathrm{C} 16$ & $1.499(10)$ \\
\hline $\mathrm{Cl3}$ & $\mathrm{C} 22$ & $1.733(8)$ & $\mathrm{C} 16$ & $\mathrm{C} 17$ & $1.413(10)$ \\
\hline $\mathrm{Cl} 4$ & $\mathrm{C} 20$ & $1.745(7)$ & $\mathrm{C} 17$ & $\mathrm{C} 18$ & $1.343(11)$ \\
\hline $\mathrm{O} 1$ & C33 & $1.290(8)$ & $\mathrm{C} 18$ & C19 & $1.426(10)$ \\
\hline $\mathrm{O} 2$ & $\mathrm{C} 23$ & $1.283(8)$ & C19 & $\mathrm{C} 20$ & $1.409(10)$ \\
\hline N1 & $\mathrm{C} 26$ & $1.322(9)$ & C19 & $\mathrm{C} 24$ & $1.402(10)$ \\
\hline N1 & $\mathrm{C} 34$ & $1.370(9)$ & $\mathrm{C} 20$ & $\mathrm{C} 21$ & $1.360(11)$ \\
\hline N2 & $\mathrm{C} 16$ & $1.330(9)$ & $\mathrm{C} 21$ & $\mathrm{C} 22$ & $1.388(10)$ \\
\hline $\mathrm{N} 2$ & $\mathrm{C} 24$ & $1.378(9)$ & $\mathrm{C} 22$ & $\mathrm{C} 23$ & $1.392(10)$ \\
\hline N3 & $\mathrm{C} 12$ & $1.320(10)$ & $\mathrm{C} 23$ & $\mathrm{C} 24$ & $1.456(10)$ \\
\hline N3 & $\mathrm{C} 13$ & $1.340(10)$ & $\mathrm{C} 25$ & $\mathrm{C} 26$ & $1.496(10)$ \\
\hline N4 & $\mathrm{C} 1$ & $1.313(10)$ & $\mathrm{C} 26$ & $\mathrm{C} 27$ & $1.432(11)$ \\
\hline N4 & $\mathrm{C} 14$ & $1.353(10)$ & $\mathrm{C} 27$ & $\mathrm{C} 28$ & $1.338(11)$ \\
\hline $\mathrm{C} 1$ & $\mathrm{C} 2$ & $1.377(12)$ & $\mathrm{C} 28$ & $\mathrm{C} 29$ & $1.408(10)$ \\
\hline $\mathrm{C} 2$ & $\mathrm{C} 3$ & $1.350(12)$ & $\mathrm{C} 29$ & $\mathrm{C} 30$ & $1.407(10)$ \\
\hline $\mathrm{C} 3$ & $\mathrm{C} 4$ & $1.403(12)$ & $\mathrm{C} 29$ & C34 & $1.414(10)$ \\
\hline $\mathrm{C} 4$ & $\mathrm{C} 5$ & $1.462(11)$ & $\mathrm{C} 30$ & $\mathrm{C} 31$ & $1.350(11)$ \\
\hline $\mathrm{C} 4$ & $\mathrm{C} 14$ & $1.396(11)$ & C31 & $\mathrm{C} 32$ & $1.399(11)$ \\
\hline $\mathrm{C} 5$ & C6 & $1.523(12)$ & C32 & C33 & $1.382(10)$ \\
\hline $\mathrm{C} 5$ & $\mathrm{C} 7$ & $1.338(13)$ & C33 & C34 & $1.469(10)$ \\
\hline
\end{tabular}

Table S24. Selected bond angles $\left(^{\circ}\right)$ for $\mathbf{C o 8}$.

\begin{tabular}{|c|c|c|c|c|c|c|}
\hline \multicolumn{3}{|c|}{ Atom Atom Atom } & \multirow{2}{*}{$\frac{\text { Angle }^{\circ}}{176.69(19)}$} & \multicolumn{2}{|c|}{ Atom Atom Atom } & \multirow{2}{*}{$\frac{\text { Angle }^{\circ}}{117.3(7)}$} \\
\hline $\mathrm{O} 1$ & Col & $\mathrm{O} 2$ & & N3 & $\mathrm{C} 13 \quad \mathrm{C} 14$ & \\
\hline $\mathrm{O} 1$ & Col & N1 & $78.0(2)$ & C9 & C13 C14 & $119.7(7)$ \\
\hline $\mathrm{O} 1$ & Col & $\mathrm{N} 2$ & $101.0(2)$ & N4 & $\mathrm{C} 14 \mathrm{C} 4$ & $123.4(8)$ \\
\hline $\mathrm{O} 1$ & Co1 & N3 & $92.1(2)$ & N4 & $\mathrm{C} 14 \quad \mathrm{C} 13$ & $116.9(7)$ \\
\hline $\mathrm{O} 1$ & Col & N4 & $88.9(2)$ & $\mathrm{C} 4$ & $\mathrm{C} 14 \quad \mathrm{C} 13$ & $119.7(7)$ \\
\hline $\mathrm{O} 2$ & Col & N1 & $98.9(2)$ & N2 & $\mathrm{C} 16 \mathrm{C} 15$ & $118.0(7)$ \\
\hline $\mathrm{O} 2$ & Col & $\mathrm{N} 2$ & $77.8(2)$ & N2 & $\mathrm{C} 16 \quad \mathrm{C} 17$ & $121.8(7)$ \\
\hline
\end{tabular}




\begin{tabular}{|c|c|c|c|c|c|c|c|}
\hline $\mathrm{O} 2$ & Co1 & N3 & $91.1(2)$ & $\mathrm{C} 17$ & $\mathrm{C} 16$ & $\mathrm{C} 15$ & $120.1(7)$ \\
\hline $\mathrm{O} 2$ & Co1 & N4 & $92.5(2)$ & $\mathrm{C} 18$ & $\mathrm{C} 17$ & $\mathrm{C} 16$ & $121.1(7)$ \\
\hline $\mathrm{N} 2$ & Col & N1 & $89.4(2)$ & C17 & $\mathrm{C} 18$ & C19 & $119.4(7)$ \\
\hline N3 & Co1 & N1 & $168.0(2)$ & $\mathrm{C} 20$ & $\mathrm{C} 19$ & $\mathrm{C} 18$ & $125.1(7)$ \\
\hline N3 & Co1 & $\mathrm{N} 2$ & $99.4(2)$ & $\mathrm{C} 24$ & $\mathrm{C} 19$ & $\mathrm{C} 18$ & $116.1(7)$ \\
\hline N4 & Co1 & N1 & $95.4(2)$ & $\mathrm{C} 24$ & $\mathrm{C} 19$ & $\mathrm{C} 20$ & $118.7(7)$ \\
\hline N4 & Co1 & N2 & $169.8(2)$ & C19 & $\mathrm{C} 20$ & $\mathrm{Cl} 4$ & $119.5(6)$ \\
\hline N4 & Co1 & N3 & $77.4(2)$ & $\mathrm{C} 21$ & $\mathrm{C} 20$ & $\mathrm{Cl} 4$ & $119.5(6)$ \\
\hline $\mathrm{C} 33$ & $\mathrm{O} 1$ & Col & $116.3(4)$ & $\mathrm{C} 21$ & $\mathrm{C} 20$ & $\mathrm{C} 19$ & $121.0(7)$ \\
\hline $\mathrm{C} 23$ & $\mathrm{O} 2$ & Co1 & $115.4(4)$ & $\mathrm{C} 20$ & $\mathrm{C} 21$ & $\mathrm{C} 22$ & $120.2(7)$ \\
\hline $\mathrm{C} 26$ & N1 & Co1 & $133.1(5)$ & $\mathrm{C} 21$ & $\mathrm{C} 22$ & $\mathrm{Cl} 3$ & $118.1(6)$ \\
\hline $\mathrm{C} 26$ & N1 & C34 & $118.3(6)$ & $\mathrm{C} 21$ & $\mathrm{C} 22$ & $\mathrm{C} 23$ & $123.3(7)$ \\
\hline C34 & N1 & Co1 & 108.1(4) & $\mathrm{C} 23$ & $\mathrm{C} 22$ & $\mathrm{Cl3}$ & $118.5(6)$ \\
\hline $\mathrm{C} 16$ & $\mathrm{~N} 2$ & Co1 & $133.7(5)$ & $\mathrm{O} 2$ & $\mathrm{C} 23$ & $\mathrm{C} 22$ & $124.6(7)$ \\
\hline C16 & $\mathrm{N} 2$ & $\mathrm{C} 24$ & 117.1(6) & $\mathrm{O} 2$ & $\mathrm{C} 23$ & C24 & $120.0(6)$ \\
\hline $\mathrm{C} 24$ & N2 & Co1 & $108.8(4)$ & $\mathrm{C} 22$ & $\mathrm{C} 23$ & $\mathrm{C} 24$ & $115.4(6)$ \\
\hline $\mathrm{C} 12$ & N3 & Co1 & $127.2(5)$ & N2 & $\mathrm{C} 24$ & C19 & $124.2(6)$ \\
\hline $\mathrm{C} 12$ & N3 & $\mathrm{C} 13$ & $118.8(7)$ & N2 & $\mathrm{C} 24$ & $\mathrm{C} 23$ & $114.5(6)$ \\
\hline $\mathrm{C} 13$ & N3 & Co1 & $114.0(5)$ & C19 & $\mathrm{C} 24$ & $\mathrm{C} 23$ & $121.3(6)$ \\
\hline $\mathrm{C} 1$ & N4 & Col & $127.6(5)$ & N1 & $\mathrm{C} 26$ & $\mathrm{C} 25$ & $119.9(7)$ \\
\hline $\mathrm{C} 1$ & N4 & $\mathrm{C} 14$ & $118.0(7)$ & N1 & $\mathrm{C} 26$ & $\mathrm{C} 27$ & $120.4(7)$ \\
\hline $\mathrm{C} 14$ & N4 & Co1 & $114.5(5)$ & $\mathrm{C} 27$ & $\mathrm{C} 26$ & $\mathrm{C} 25$ & $119.5(7)$ \\
\hline N4 & $\mathrm{C} 1$ & $\mathrm{C} 2$ & $122.7(8)$ & C28 & $\mathrm{C} 27$ & C26 & $121.9(7)$ \\
\hline $\mathrm{C} 3$ & $\mathrm{C} 2$ & $\mathrm{C} 1$ & 119.7(9) & $\mathrm{C} 27$ & $\mathrm{C} 28$ & C29 & $118.9(7)$ \\
\hline $\mathrm{C} 2$ & $\mathrm{C} 3$ & $\mathrm{C} 4$ & $120.1(8)$ & C28 & $\mathrm{C} 29$ & C34 & $117.0(7)$ \\
\hline $\mathrm{C} 3$ & $\mathrm{C} 4$ & $\mathrm{C} 5$ & $124.2(8)$ & $\mathrm{C} 30$ & $\mathrm{C} 29$ & C28 & $125.1(7)$ \\
\hline C14 & $\mathrm{C} 4$ & C3 & $116.0(8)$ & $\mathrm{C} 30$ & $\mathrm{C} 29$ & C34 & $118.0(7)$ \\
\hline $\mathrm{C} 14$ & $\mathrm{C} 4$ & $\mathrm{C} 5$ & $119.8(8)$ & C29 & $\mathrm{C} 30$ & $\mathrm{Cl1}$ & $118.7(6)$ \\
\hline $\mathrm{C} 4$ & $\mathrm{C} 5$ & C6 & 116.3(8) & C31 & $\mathrm{C} 30$ & $\mathrm{Cl1}$ & $119.2(6)$ \\
\hline $\mathrm{C} 7$ & $\mathrm{C} 5$ & $\mathrm{C} 4$ & $120.3(8)$ & C31 & $\mathrm{C} 30$ & C29 & $122.1(7)$ \\
\hline $\mathrm{C} 7$ & $\mathrm{C} 5$ & C6 & $123.4(8)$ & $\mathrm{C} 30$ & C31 & C32 & $119.7(8)$ \\
\hline $\mathrm{C} 5$ & $\mathrm{C} 7$ & $\mathrm{C} 8$ & $122.9(8)$ & C31 & $\mathrm{C} 32$ & $\mathrm{Cl} 2$ & $117.7(6)$ \\
\hline $\mathrm{C} 5$ & $\mathrm{C} 7$ & $\mathrm{C} 9$ & $121.0(8)$ & $\mathrm{C} 33$ & $\mathrm{C} 32$ & $\mathrm{Cl} 2$ & $118.6(6)$ \\
\hline $\mathrm{C} 9$ & $\mathrm{C} 7$ & $\mathrm{C} 8$ & $116.0(8)$ & C33 & $\mathrm{C} 32$ & C31 & $123.7(7)$ \\
\hline $\mathrm{C} 10$ & C9 & $\mathrm{C} 7$ & $123.3(8)$ & $\mathrm{O} 1$ & $\mathrm{C} 33$ & C32 & $124.8(7)$ \\
\hline C10 & C9 & C13 & $117.2(8)$ & $\mathrm{O} 1$ & C33 & C34 & $120.0(6)$ \\
\hline $\mathrm{C} 13$ & C9 & $\mathrm{C} 7$ & $119.5(8)$ & C32 & $\mathrm{C} 33$ & C34 & $115.2(7)$ \\
\hline C11 & $\mathrm{C} 10$ & C9 & 119.1(8) & N1 & $\mathrm{C} 34$ & C29 & $123.4(6)$ \\
\hline $\mathrm{C} 10$ & C11 & C12 & 119.9(9) & N1 & C34 & C33 & $115.4(6)$ \\
\hline N3 & $\mathrm{C} 12$ & $\mathrm{C} 11$ & $121.7(8)$ & C29 & $\mathrm{C} 34$ & C33 & $121.1(7)$ \\
\hline $\mathrm{N} 3$ & $\mathrm{C} 13$ & $\mathrm{C} 9$ & $123.1(8)$ & & & & \\
\hline
\end{tabular}


Table S25. Crystal data and structure refinement details for Co9.

\begin{tabular}{|c|c|}
\hline Empirical formula & $\mathrm{C}_{33} \mathrm{H}_{26} \mathrm{Cl}_{4} \mathrm{CoN}_{4} \mathrm{O}_{5}$ \\
\hline Formula weight & 759.31 \\
\hline Temperature/K & 293(2) \\
\hline Crystal system & monoclinic \\
\hline Space group & $\mathrm{P} 2{ }_{1} / \mathrm{c}$ \\
\hline $\mathrm{a} / \AA ̊$ & $14.7387(6)$ \\
\hline $\mathrm{b} / \AA$ & $13.5042(5)$ \\
\hline $\mathrm{c} / \AA$ & $17.1257(6)$ \\
\hline$\alpha /^{\circ}$ & 90 \\
\hline$\beta /{ }^{\circ}$ & $94.8950(10)$ \\
\hline$\gamma /{ }^{\circ}$ & 90 \\
\hline Volume $/ \AA^{3}$ & $3396.2(2)$ \\
\hline $\mathrm{Z}$ & 4 \\
\hline$\rho_{\text {calc }} \mathrm{mg} / \mathrm{mm}^{3}$ & 1.485 \\
\hline $\mathrm{m} / \mathrm{mm}^{-1}$ & 0.867 \\
\hline $\mathrm{F}(000)$ & 1548.0 \\
\hline Crystal size $/ \mathrm{mm}^{3}$ & $0.225 \times 0.187 \times 0.112$ \\
\hline $2 \Theta$ range for data collection & 5.648 to $50.7^{\circ}$ \\
\hline Index ranges & $-17 \leq \mathrm{h} \leq 17,-16 \leq \mathrm{k} \leq 16,-19 \leq 1 \leq 19$ \\
\hline Reflections collected & 45716 \\
\hline Independent reflections & $6065[\mathrm{R}(\mathrm{int})=0.0732]$ \\
\hline Data/restraints/parameters & $6065 / 1422 / 440$ \\
\hline Goodness-of-fit on $\mathrm{F}^{2}$ & 1.037 \\
\hline Final $\mathrm{R}$ indexes $[\mathrm{I}>=2 \sigma(\mathrm{I})]$ & $\mathrm{R}_{1}=0.0767, \mathrm{wR}_{2}=0.2022$ \\
\hline Final R indexes [all data] & $\mathrm{R}_{1}=0.1287, \mathrm{wR}_{2}=0.2334$ \\
\hline Largest diff. peak/hole / e $\AA^{-3}$ & $30.88 /-1.01$ \\
\hline
\end{tabular}

Table S26. Selected bond lengths ( $\AA$ ) for Co9.

\begin{tabular}{|c|c|c|c|c|c|}
\hline \multicolumn{2}{|c|}{ Atom Atom } & \multirow{2}{*}{$\frac{\text { Length/Å }}{2.020(4)}$} & \multicolumn{2}{|c|}{ Atom Atom } & \multirow{2}{*}{$\frac{\text { Length/Å }}{1.391(8)}$} \\
\hline Col & O1 & & $\mathrm{C} 7$ & $\mathrm{~N} 2$ & \\
\hline Col & $\mathrm{O} 2$ & $2.011(4)$ & $\mathrm{C} 7$ & $\mathrm{C} 8$ & $1.315(8)$ \\
\hline Col & N3 & $2.128(5)$ & $\mathrm{C} 12$ & $\mathrm{~N} 2$ & $1.524(9)$ \\
\hline Col & N1 & $2.227(5)$ & $\mathrm{C} 12$ & $\mathrm{C} 11$ & $1.268(9)$ \\
\hline Col & $\mathrm{N} 2$ & $1.980(5)$ & $\mathrm{C} 13$ & $\mathrm{C} 14$ & $1.374(8)$ \\
\hline $\mathrm{Cl1}$ & $\mathrm{C} 24$ & $1.748(7)$ & C13 & $\mathrm{C} 22$ & $1.467(9)$ \\
\hline $\mathrm{Cl} 2$ & $\mathrm{C} 26$ & $1.533(6)$ & $\mathrm{C} 14$ & $\mathrm{C} 15$ & $1.390(9)$ \\
\hline $\mathrm{Cl} 3$ & $\mathrm{C} 16$ & $1.716(7)$ & $\mathrm{C} 15$ & $\mathrm{C} 16$ & $1.378(10)$ \\
\hline $\mathrm{Cl} 4$ & $\mathrm{C} 14$ & $1.803(7)$ & $\mathrm{C} 16$ & $\mathrm{C} 17$ & $1.403(9)$ \\
\hline
\end{tabular}




\begin{tabular}{cccccc}
\hline $\mathrm{O} 1$ & $\mathrm{C} 13$ & $1.292(7)$ & $\mathrm{C} 17$ & $\mathrm{C} 18$ & $1.446(10)$ \\
$\mathrm{O} 2$ & $\mathrm{C} 23$ & $1.130(6)$ & $\mathrm{C} 17$ & $\mathrm{C} 22$ & $1.398(9)$ \\
$\mathrm{O} 3$ & $\mathrm{C} 3$ & $1.431(9)$ & $\mathrm{C} 18$ & $\mathrm{C} 19$ & $1.337(10)$ \\
$\mathrm{O} 3$ & $\mathrm{C} 4$ & $1.425(18)$ & $\mathrm{C} 19$ & $\mathrm{C} 20$ & $1.399(10)$ \\
$\mathrm{O} 3$ & $\mathrm{C} 4$ & $1.256(9)$ & $\mathrm{C} 20$ & $\mathrm{C} 21$ & $1.479(10)$ \\
$\mathrm{O} 4$ & $\mathrm{C} 10$ & $1.583(9)$ & $\mathrm{C} 23$ & $\mathrm{C} 24$ & $1.344(9)$ \\
$\mathrm{O} 4$ & $\mathrm{C} 9$ & $1.232(7)$ & $\mathrm{C} 23$ & $\mathrm{C} 32$ & $1.533(7)$ \\
$\mathrm{N} 3$ & $\mathrm{C} 30$ & $1.342(8)$ & $\mathrm{C} 24$ & $\mathrm{C} 25$ & $1.234(9)$ \\
$\mathrm{N} 3$ & $\mathrm{C} 32$ & $1.307(7)$ & $\mathrm{C} 25$ & $\mathrm{C} 26$ & $1.332(9)$ \\
$\mathrm{N} 4$ & $\mathrm{C} 20$ & $1.395(9)$ & $\mathrm{C} 26$ & $\mathrm{C} 27$ & $1.410(9)$ \\
$\mathrm{N} 4$ & $\mathrm{C} 22$ & $1.378(8)$ & $\mathrm{C} 27$ & $\mathrm{C} 28$ & $1.417(9)$ \\
$\mathrm{C} 1$ & $\mathrm{~N} 1$ & $1.537(9)$ & $\mathrm{C} 27$ & $\mathrm{C} 32$ & $1.228(8)$ \\
$\mathrm{C} 1$ & $\mathrm{C} 2$ & $1.437(10)$ & $\mathrm{C} 28$ & $\mathrm{C} 29$ & $1.337(10)$ \\
$\mathrm{N} 1$ & $\mathrm{C} 6$ & $1.217(7)$ & $\mathrm{C} 29$ & $\mathrm{C} 30$ & $1.240(8)$ \\
$\mathrm{C} 6$ & $\mathrm{C} 5$ & $1.484(9)$ & $\mathrm{C} 30$ & $\mathrm{C} 31$ & $1.494(9)$ \\
$\mathrm{C} 6$ & $\mathrm{C} 7$ & $1.542(9)$ & $\mathrm{C} 33$ & $\mathrm{O} 5$ & $1.354(14)$ \\
$\mathrm{C} 5$ & $\mathrm{C} 3$ & $1.575(10)$ & $\mathrm{C} 8$ & $\mathrm{C} 9$ & $1.530(9)$ \\
$\mathrm{C} 3$ & $\mathrm{C} 2$ & $1.226(9)$ & $\mathrm{C} 9$ & $\mathrm{C} 11$ & $1.385(9)$ \\
\hline
\end{tabular}

Table S27. Selected bond angles $\left(^{\circ}\right)$ for $\mathbf{C o 9}$.

\begin{tabular}{|c|c|c|c|c|c|c|c|}
\hline \multicolumn{3}{|c|}{ Atom Atom Atom } & \multirow{2}{*}{$\frac{\text { Angle }^{\circ}}{97.65(18)}$} & \multicolumn{3}{|c|}{ Atom Atom Atom } & \multirow{2}{*}{$\frac{\text { Angle }^{\circ}}{118.9(5)}$} \\
\hline $\mathrm{O} 1$ & $\mathrm{Co} 1$ & N3 & & $\mathrm{C} 15$ & $\mathrm{C} 14$ & $\mathrm{Cl} 4$ & \\
\hline $\mathrm{O} 1$ & Col & N1 & $93.71(17)$ & $\mathrm{C} 16$ & $\mathrm{C} 15$ & C14 & $120.1(6)$ \\
\hline $\mathrm{O} 2$ & Co1 & O1 & $174.39(15)$ & $\mathrm{C} 15$ & $\mathrm{C} 16$ & $\mathrm{Cl3}$ & $118.8(5)$ \\
\hline $\mathrm{O} 2$ & Col & N3 & $80.00(18)$ & $\mathrm{C} 15$ & $\mathrm{C} 16$ & $\mathrm{C} 17$ & $124.0(6)$ \\
\hline $\mathrm{O} 2$ & Col & N1 & $91.06(18)$ & $\mathrm{C} 17$ & $\mathrm{C} 16$ & $\mathrm{Cl} 3$ & $117.3(6)$ \\
\hline N3 & Col & N1 & $82.85(19)$ & $\mathrm{C} 16$ & $\mathrm{C} 17$ & $\mathrm{C} 18$ & $127.2(6)$ \\
\hline $\mathrm{N} 2$ & Co 1 & $\mathrm{O} 1$ & $93.66(18)$ & $\mathrm{C} 22$ & $\mathrm{C} 17$ & $\mathrm{C} 16$ & $115.1(6)$ \\
\hline $\mathrm{N} 2$ & Col & $\mathrm{O} 2$ & $89.08(18)$ & $\mathrm{C} 22$ & $\mathrm{C} 17$ & $\mathrm{C} 18$ & $117.8(6)$ \\
\hline N2 & Col & N3 & $168.00(19)$ & C19 & $\mathrm{C} 18$ & $\mathrm{C} 17$ & $123.1(7)$ \\
\hline N2 & Col & N1 & $92.5(2)$ & $\mathrm{C} 18$ & $\mathrm{C} 19$ & $\mathrm{C} 20$ & $117.8(7)$ \\
\hline $\mathrm{C} 13$ & $\mathrm{O} 1$ & Col & $116.8(4)$ & N4 & $\mathrm{C} 20$ & $\mathrm{C} 19$ & $120.9(6)$ \\
\hline $\mathrm{C} 23$ & $\mathrm{O} 2$ & Co1 & $116.4(4)$ & N4 & $\mathrm{C} 20$ & $\mathrm{C} 21$ & $124.5(6)$ \\
\hline $\mathrm{C}^{\prime}$ & $\mathrm{O} 3$ & $\mathrm{C} 3$ & $146.5(12)$ & C19 & $\mathrm{C} 20$ & $\mathrm{C} 21$ & $114.5(7)$ \\
\hline $\mathrm{C} 4$ & $\mathrm{O} 3$ & C3 & $108.9(7)$ & N4 & $\mathrm{C} 22$ & $\mathrm{C} 13$ & $118.6(5)$ \\
\hline C9 & $\mathrm{O} 4$ & $\mathrm{C} 10$ & $124.1(6)$ & N4 & $\mathrm{C} 22$ & $\mathrm{C} 17$ & $119.0(6)$ \\
\hline $\mathrm{C} 30$ & N3 & Col & $132.8(4)$ & $\mathrm{C} 17$ & $\mathrm{C} 22$ & C13 & $122.4(6)$ \\
\hline C32 & N3 & Co1 & $101.8(4)$ & $\mathrm{O} 2$ & $\mathrm{C} 23$ & $\mathrm{C} 24$ & $124.9(6)$ \\
\hline $\mathrm{C} 32$ & N3 & C30 & $124.4(5)$ & $\mathrm{O} 2$ & $\mathrm{C} 23$ & C32 & $112.5(5)$ \\
\hline $\mathrm{C} 22$ & N4 & $\mathrm{C} 20$ & $121.1(5)$ & $\mathrm{C} 24$ & $\mathrm{C} 23$ & C32 & $122.5(5)$ \\
\hline $\mathrm{C} 2$ & $\mathrm{C} 1$ & N1 & $136.0(5)$ & $\mathrm{C} 23$ & C24 & $\mathrm{Cl1}$ & $124.4(5)$ \\
\hline
\end{tabular}




\begin{tabular}{cccccccc}
\hline C1 & N1 & Co1 & $137.5(3)$ & C25 & C24 & C11 & $115.1(6)$ \\
C6 & N1 & Co1 & $100.2(5)$ & C25 & C24 & C23 & $120.5(7)$ \\
C6 & N1 & C1 & $122.2(5)$ & C24 & C25 & C26 & $116.5(7)$ \\
N1 & C6 & C5 & $104.0(6)$ & C25 & C26 & C12 & $115.2(5)$ \\
N1 & C6 & C7 & $120.4(5)$ & C25 & C26 & C27 & $128.8(6)$ \\
C5 & C6 & C7 & $135.5(5)$ & C27 & C26 & C12 & $116.0(5)$ \\
C6 & C5 & C3 & $132.1(5)$ & C26 & C27 & C28 & $132.5(6)$ \\
O3 & C3 & C5 & $136.3(5)$ & C32 & C27 & C26 & $117.0(6)$ \\
C2 & C3 & O3 & $101.8(7)$ & C32 & C27 & C28 & $110.4(6)$ \\
C2 & C3 & C5 & $121.9(6)$ & C29 & C28 & C27 & $127.8(6)$ \\
C3 & C2 & C1 & $103.7(7)$ & C30 & C29 & C28 & $115.7(7)$ \\
N2 & C7 & C6 & $129.5(5)$ & N3 & C30 & C31 & $128.0(5)$ \\
C8 & C7 & C6 & $130.8(5)$ & C29 & C30 & N3 & $118.2(6)$ \\
C8 & C7 & N2 & $99.7(6)$ & C29 & C30 & C31 & $113.8(6)$ \\
C11 & C12 & N2 & $129.3(6)$ & N3 & C32 & C23 & $122.1(5)$ \\
C7 & N2 & Co1 & $96.6(4)$ & C27 & C32 & N3 & $123.3(6)$ \\
C7 & N2 & C12 & $131.4(4)$ & C27 & C32 & C23 & $114.6(6)$ \\
C12 & N2 & Co1 & $131.7(3)$ & C7 & C8 & C9 & $127.9(6)$ \\
O1 & C13 & C14 & $121.3(6)$ & O4 & C9 & C8 & $122.0(6)$ \\
O1 & C13 & C22 & $120.8(5)$ & O4 & C9 & C11 & $106.8(6)$ \\
C14 & C13 & C22 & $117.9(5)$ & C11 & C9 & C8 & $131.1(5)$ \\
C13 & C14 & C14 & $120.6(5)$ & C12 & C11 & C9 & $100.6(7)$ \\
C13 & C14 & C15 & $120.5(6)$ & & & & \\
\hline & & & & & & & \\
\hline & & & & & \\
\end{tabular}

Table S28. Crystal data and structure refinement details for Co10.

\begin{tabular}{ll}
\hline Empirical formula & $\mathrm{C}_{89} \mathrm{H}_{60} \mathrm{Cl}_{8} \mathrm{Co}_{2} \mathrm{~N}_{8} \mathrm{O}_{5}$ \\
Formula weight & 1722.91 \\
Temperature/K & $293(2)$ \\
Crystal system & monoclinic \\
Space group & $\mathrm{P} 2_{1}$ \\
$\mathrm{a} / \AA$ & $14.5503(6)$ \\
$\mathrm{b} / \AA$ & $15.7208(6)$ \\
$\mathrm{c} / \AA$ & $17.5652(7)$ \\
$\alpha /{ }^{\circ}$ & 90 \\
$\beta /{ }^{\circ}$ & $96.7990(10)$ \\
$\gamma /{ }^{\circ}$ & 90 \\
Volume $/ \AA^{3}$ & $3989.6(3)$ \\
$\mathrm{Z}$ & 2 \\
$\rho_{\text {calc }} / \mathrm{cm}^{3}$ & 1.434 \\
$\mu / \mathrm{mm}^{-1}$ & 0.743 \\
$\mathrm{~F}(000)$ & 1760.0 \\
Crystal size $/ \mathrm{mm}^{3}$ & $0.3 \times 0.2 \times 0.11$ \\
\hline & \multicolumn{2}{c}{$\mathrm{S} 26$}
\end{tabular}




\begin{tabular}{ll}
\hline Radiation & $\operatorname{MoK} \alpha(\lambda=0.71073)$ \\
$2 \Theta$ range for data collection/ & 5.736 to 55.124 \\
Index ranges & $-18 \leq \mathrm{h} \leq 18,-20 \leq \mathrm{k} \leq 20,-22 \leq 1 \leq 22$ \\
Reflections collected & 63870 \\
Independent reflections & $18298\left[\mathrm{R}_{\text {int }}=0.0949, \mathrm{R}_{\text {sigma }}=0.1586\right]$ \\
Data/restraints/parameters & $18298 / 398 / 896$ \\
Goodness-of-fit on $\mathrm{F}^{2}$ & 0.897 \\
Final R indexes [I $>=2 \sigma(\mathrm{I})]$ & $\mathrm{R}_{1}=0.0847, \mathrm{wR}_{2}=0.2150$ \\
Final R indexes [all data] & $\mathrm{R}_{1}=0.2190, \mathrm{wR}_{2}=0.2892$ \\
Largest diff. peak/hole / e $\AA^{-3} 0.95 /-0.35$ \\
Flack parameter & $0.32(3)$ \\
\hline
\end{tabular}

Table S29. Selected bond lengths ( $\AA$ ) for Co10.

\begin{tabular}{|c|c|c|c|c|c|}
\hline \multicolumn{2}{|c|}{ Atom Atom } & \multirow{2}{*}{$\frac{\text { Length/Å }}{2.044(8)}$} & \multicolumn{2}{|c|}{ Atom Atom } & \multirow{2}{*}{$\frac{\text { Length/Å }}{1.479(13)}$} \\
\hline $\mathrm{Co} 2$ & $\mathrm{O5}$ & & C39 & $\mathrm{C} 40$ & \\
\hline $\mathrm{Co} 2$ & N6 & $2.208(11)$ & $\mathrm{C} 31$ & $\mathrm{C} 30$ & 1.3900 \\
\hline $\mathrm{Co} 2$ & N5 & $2.152(10)$ & $\mathrm{C} 31$ & $\mathrm{C} 32$ & 1.3900 \\
\hline $\mathrm{Co} 2$ & N7 & $2.257(11)$ & $\mathrm{C} 30$ & $\mathrm{C} 29$ & 1.3900 \\
\hline $\mathrm{Co} 2$ & N8 & $2.163(10)$ & $\mathrm{C} 29$ & $\mathrm{C} 28$ & 1.3900 \\
\hline $\mathrm{Co} 2$ & $\mathrm{O} 4$ & $2.019(9)$ & $\mathrm{C} 28$ & $\mathrm{C} 27$ & 1.3900 \\
\hline Col & N3 & $2.100(10)$ & $\mathrm{C} 27$ & $\mathrm{C} 32$ & 1.3900 \\
\hline Col & $\mathrm{O} 3$ & $2.011(10)$ & $\mathrm{C} 27$ & $\mathrm{C} 24$ & $1.480(14)$ \\
\hline Col & $\mathrm{O} 2$ & $2.016(9)$ & $\mathrm{C} 23$ & $\mathrm{C} 24$ & $1.369(17)$ \\
\hline Col & $\mathrm{N} 2$ & $2.230(11)$ & C71 & $\mathrm{C} 72$ & $1.40(2)$ \\
\hline Co1 & N4 & $2.129(10)$ & C71 & $\mathrm{C} 70$ & $1.52(2)$ \\
\hline Co1 & N1 & $2.190(11)$ & $\mathrm{C} 37$ & C38 & $1.407(18)$ \\
\hline $\mathrm{Cl} 5$ & $\mathrm{C} 87$ & $1.703(7)$ & C6 & $\mathrm{C} 11$ & 1.3900 \\
\hline $\mathrm{Cl} 18$ & $\mathrm{C} 77$ & $1.694(8)$ & C6 & $\mathrm{C} 7$ & 1.3900 \\
\hline $\mathrm{Cl} 7$ & C79 & $1.757(7)$ & C6 & $\mathrm{C} 5$ & $1.410(16)$ \\
\hline $\mathrm{Cl1}$ & C9 & $1.729(8)$ & $\mathrm{C} 11$ & $\mathrm{C} 10$ & 1.3900 \\
\hline $\mathrm{Cl} 6$ & $\mathrm{C} 85$ & $1.749(6)$ & $\mathrm{C} 10$ & C9 & 1.3900 \\
\hline $\mathrm{Cl} 2$ & $\mathrm{C} 11$ & $1.740(8)$ & C9 & $\mathrm{C} 8$ & 1.3900 \\
\hline $\mathrm{Cl} 4$ & C19 & $1.732(12)$ & $\mathrm{C} 8$ & $\mathrm{C} 7$ & 1.3900 \\
\hline O5 & C88 & $1.337(9)$ & C75 & $\mathrm{C} 74$ & 1.3900 \\
\hline N3 & $\mathrm{C} 36$ & $1.353(11)$ & C75 & C76 & 1.3900 \\
\hline N3 & $\mathrm{C} 37$ & $1.343(15)$ & C74 & C79 & 1.3900 \\
\hline N6 & C89 & $1.386(11)$ & C74 & C73 & $1.492(16)$ \\
\hline N6 & $\mathrm{C} 81$ & $1.347(17)$ & C79 & C78 & 1.3900 \\
\hline N5 & C55 & $1.372(10)$ & C78 & C77 & 1.3900 \\
\hline N5 & $\mathrm{C} 54$ & $1.292(15)$ & C77 & C76 & 1.3900 \\
\hline $\mathrm{O} 3$ & $\mathrm{C} 8$ & $1.344(12)$ & N1 & $\mathrm{C} 13$ & $1.30(2)$ \\
\hline $\mathrm{O} 2$ & $\mathrm{C} 18$ & $1.328(11)$ & N1 & $\mathrm{C} 17$ & $1.387(16)$ \\
\hline N7 & C71 & $1.326(17)$ & $\mathrm{C} 3$ & $\mathrm{C} 2$ & $1.464(19)$ \\
\hline N7 & $\mathrm{C} 75$ & $1.394(12)$ & $\mathrm{C} 3$ & $\mathrm{C} 4$ & $1.432(19)$ \\
\hline
\end{tabular}




\begin{tabular}{|c|c|c|c|c|c|}
\hline $\mathrm{N} 2$ & $\mathrm{C} 7$ & $1.363(12)$ & C69 & C68 & 1.3900 \\
\hline $\mathrm{N} 2$ & $\mathrm{C} 3$ & $1.330(15)$ & C69 & C64 & 1.3900 \\
\hline N8 & C60 & $1.368(10)$ & C68 & C67 & 1.3900 \\
\hline N8 & C61 & $1.323(16)$ & C67 & C66 & 1.3900 \\
\hline $\mathrm{O} 4$ & $\mathrm{C} 76$ & $1.343(11)$ & C66 & $\mathrm{C} 65$ & 1.3900 \\
\hline C52 & C56 & $1.478(12)$ & C65 & C64 & 1.3900 \\
\hline C52 & $\mathrm{C} 53$ & $1.361(16)$ & $\mathrm{C} 72$ & $\mathrm{C} 73$ & $1.31(2)$ \\
\hline C52 & C51 & $1.482(12)$ & C61 & C62 & $1.353(18)$ \\
\hline $\mathrm{N} 4$ & $\mathrm{C} 26$ & $1.370(10)$ & $\mathrm{C} 81$ & $\mathrm{C} 82$ & $1.39(2)$ \\
\hline $\mathrm{N} 4$ & $\mathrm{C} 22$ & $1.333(15)$ & C81 & $\mathrm{C} 80$ & $1.44(2)$ \\
\hline C34 & $\mathrm{C} 35$ & 1.3900 & $\mathrm{C} 45$ & $\mathrm{C} 44$ & 1.3900 \\
\hline C34 & $\mathrm{C} 33$ & 1.3900 & $\mathrm{C} 45$ & $\mathrm{C} 40$ & 1.3900 \\
\hline C35 & $\mathrm{C} 36$ & 1.3900 & $\mathrm{C} 44$ & $\mathrm{C} 43$ & 1.3900 \\
\hline C35 & C39 & $1.473(13)$ & $\mathrm{C} 43$ & $\mathrm{C} 42$ & 1.3900 \\
\hline C36 & $\mathrm{C} 26$ & 1.3900 & $\mathrm{C} 42$ & $\mathrm{C} 41$ & 1.3900 \\
\hline C26 & $\mathrm{C} 25$ & 1.3900 & $\mathrm{C} 41$ & $\mathrm{C} 40$ & 1.3900 \\
\hline $\mathrm{C} 25$ & $\mathrm{C} 33$ & 1.3900 & $\mathrm{C} 4$ & $\mathrm{C} 5$ & $1.38(2)$ \\
\hline $\mathrm{C} 25$ & $\mathrm{C} 24$ & $1.498(13)$ & $\mathrm{C} 83$ & $\mathrm{C} 82$ & $1.40(2)$ \\
\hline $\mathrm{C} 22$ & $\mathrm{C} 23$ & $1.395(18)$ & $\mathrm{C} 14$ & $\mathrm{C} 13$ & $1.52(3)$ \\
\hline C56 & C55 & 1.3900 & $\mathrm{C} 14$ & $\mathrm{C} 15$ & $1.42(3)$ \\
\hline C56 & $\mathrm{C} 57$ & 1.3900 & $\mathrm{C} 47$ & $\mathrm{C} 46$ & 1.3900 \\
\hline C55 & C60 & 1.3900 & $\mathrm{C} 47$ & $\mathrm{C} 48$ & 1.3900 \\
\hline C60 & C59 & 1.3900 & $\mathrm{C} 46$ & C51 & 1.3900 \\
\hline C59 & $\mathrm{C} 58$ & 1.3900 & C51 & $\mathrm{C} 50$ & 1.3900 \\
\hline C59 & C63 & $1.479(13)$ & C50 & $\mathrm{C} 49$ & 1.3900 \\
\hline C58 & $\mathrm{C} 57$ & 1.3900 & $\mathrm{C} 49$ & $\mathrm{C} 48$ & 1.3900 \\
\hline C53 & $\mathrm{C} 54$ & $1.405(16)$ & $\mathrm{C} 13$ & $\mathrm{C} 12$ & $1.44(3)$ \\
\hline C88 & $\mathrm{C} 89$ & 1.3900 & $\mathrm{C} 21$ & $\mathrm{C} 20$ & 1.3900 \\
\hline C88 & $\mathrm{C} 87$ & 1.3900 & $\mathrm{C} 21$ & $\mathrm{C} 16$ & 1.3900 \\
\hline C89 & $\mathrm{C} 84$ & 1.3900 & $\mathrm{C} 21$ & $\mathrm{Cl} 3$ & $1.746(9)$ \\
\hline C84 & $\mathrm{C} 85$ & 1.3900 & $\mathrm{C} 20$ & $\mathrm{C} 19$ & 1.3900 \\
\hline C84 & $\mathrm{C} 83$ & $1.420(17)$ & C19 & $\mathrm{C} 18$ & 1.3900 \\
\hline $\mathrm{C} 85$ & $\mathrm{C} 86$ & 1.3900 & $\mathrm{C} 18$ & $\mathrm{C} 17$ & 1.3900 \\
\hline $\mathrm{C} 86$ & $\mathrm{C} 87$ & 1.3900 & $\mathrm{C} 17$ & $\mathrm{C} 16$ & 1.3900 \\
\hline C63 & C64 & $1.494(14)$ & $\mathrm{C} 16$ & $\mathrm{C} 15$ & $1.30(2)$ \\
\hline C63 & C62 & $1.372(17)$ & $\mathrm{O} 1$ & $\mathrm{C} 1$ & $1.37(2)$ \\
\hline C39 & $\mathrm{C} 38$ & $1.368(18)$ & & & \\
\hline
\end{tabular}

Table S30. Selected bond angles $\left(^{\circ}\right)$ for Co10.

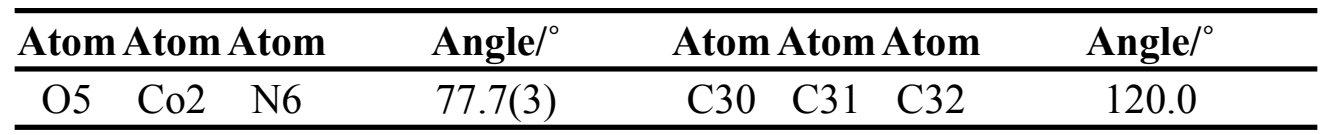




\begin{tabular}{|c|c|c|c|c|c|c|c|}
\hline O5 & $\mathrm{Co} 2$ & N5 & $94.3(3)$ & $\mathrm{C} 29$ & $\mathrm{C} 30$ & $\mathrm{C} 31$ & 120.0 \\
\hline O5 & $\mathrm{Co} 2$ & N7 & $104.8(3)$ & $\mathrm{C} 28$ & $\mathrm{C} 29$ & $\mathrm{C} 30$ & 120.0 \\
\hline $\mathrm{O} 5$ & $\mathrm{Co} 2$ & N8 & $88.2(3)$ & $\mathrm{C} 29$ & $\mathrm{C} 28$ & $\mathrm{C} 27$ & 120.0 \\
\hline N6 & $\mathrm{Co} 2$ & N7 & 98.1(4) & $\mathrm{C} 28$ & $\mathrm{C} 27$ & $\mathrm{C} 24$ & $118.4(7)$ \\
\hline N5 & $\mathrm{Co} 2$ & N6 & $96.4(4)$ & C32 & $\mathrm{C} 27$ & $\mathrm{C} 28$ & 120.0 \\
\hline N5 & $\mathrm{Co} 2$ & N7 & $158.1(4)$ & $\mathrm{C} 32$ & $\mathrm{C} 27$ & $\mathrm{C} 24$ & $121.3(7)$ \\
\hline N5 & $\mathrm{Co} 2$ & N8 & $76.4(4)$ & $\mathrm{C} 27$ & $\mathrm{C} 32$ & $\mathrm{C} 31$ & 120.0 \\
\hline N8 & $\mathrm{Co} 2$ & N6 & $163.7(4)$ & $\mathrm{C} 24$ & $\mathrm{C} 23$ & $\mathrm{C} 22$ & $120.7(12)$ \\
\hline N8 & $\mathrm{Co} 2$ & N7 & 93.3(4) & N7 & C71 & $\mathrm{C} 72$ & $120.8(14)$ \\
\hline $\mathrm{O} 4$ & $\mathrm{Co} 2$ & O5 & $176.2(4)$ & N7 & C71 & $\mathrm{C} 70$ & $118.2(13)$ \\
\hline $\mathrm{O} 4$ & $\mathrm{Co} 2$ & N6 & $104.1(4)$ & C72 & C71 & $\mathrm{C} 70$ & $121.0(13)$ \\
\hline $\mathrm{O} 4$ & $\mathrm{Co} 2$ & N5 & $82.2(4)$ & $\mathrm{C} 27$ & $\mathrm{C} 24$ & $\mathrm{C} 25$ & $123.5(8)$ \\
\hline $\mathrm{O} 4$ & $\mathrm{Co} 2$ & N7 & 78.4(4) & $\mathrm{C} 23$ & $\mathrm{C} 24$ & $\mathrm{C} 25$ & $116.2(10)$ \\
\hline $\mathrm{O} 4$ & $\mathrm{Co} 2$ & N8 & $89.6(4)$ & $\mathrm{C} 23$ & $\mathrm{C} 24$ & $\mathrm{C} 27$ & $120.3(10)$ \\
\hline N3 & Col & N2 & $94.2(4)$ & N3 & $\mathrm{C} 37$ & $\mathrm{C} 38$ & $122.3(12)$ \\
\hline $\mathrm{N} 3$ & Co1 & N4 & $77.0(4)$ & $\mathrm{C} 11$ & C6 & $\mathrm{C} 7$ & 120.0 \\
\hline N3 & Col & N1 & $168.9(5)$ & $\mathrm{C} 11$ & C6 & $\mathrm{C} 5$ & $121.8(8)$ \\
\hline $\mathrm{O} 3$ & Col & N3 & $87.8(4)$ & $\mathrm{C} 7$ & C6 & $\mathrm{C} 5$ & $118.2(8)$ \\
\hline $\mathrm{O} 3$ & Co1 & $\mathrm{O} 2$ & $177.3(4)$ & C6 & $\mathrm{C} 11$ & $\mathrm{Cl} 2$ & $121.2(6)$ \\
\hline $\mathrm{O} 3$ & Col & N2 & $78.6(4)$ & C6 & $\mathrm{C} 11$ & $\mathrm{C} 10$ & 120.0 \\
\hline $\mathrm{O} 3$ & Co1 & N4 & $90.4(4)$ & $\mathrm{C} 10$ & $\mathrm{C} 11$ & $\mathrm{Cl} 2$ & $118.8(6)$ \\
\hline $\mathrm{O} 3$ & Co1 & N1 & $103.0(5)$ & $\mathrm{C} 11$ & $\mathrm{C} 10$ & C9 & 120.0 \\
\hline $\mathrm{O} 2$ & Col & N3 & $90.0(4)$ & $\mathrm{C} 10$ & C9 & $\mathrm{Cl1}$ & $118.6(6)$ \\
\hline $\mathrm{O} 2$ & Col & N2 & $103.1(4)$ & $\mathrm{C} 8$ & C9 & $\mathrm{C} 11$ & $121.3(6)$ \\
\hline $\mathrm{O} 2$ & Co1 & N4 & $87.7(4)$ & $\mathrm{C} 8$ & C9 & $\mathrm{C} 10$ & 120.0 \\
\hline $\mathrm{O} 2$ & Co1 & N1 & $79.1(5)$ & $\mathrm{O} 3$ & $\mathrm{C} 8$ & C9 & $119.4(7)$ \\
\hline N4 & Co1 & N2 & $166.2(4)$ & $\mathrm{O} 3$ & $\mathrm{C} 8$ & $\mathrm{C} 7$ & $120.5(7)$ \\
\hline N4 & Co1 & N1 & $100.3(4)$ & $\mathrm{C} 7$ & $\mathrm{C} 8$ & C9 & 120.0 \\
\hline $\mathrm{N} 1$ & Col & N2 & $90.3(4)$ & N2 & $\mathrm{C} 7$ & C6 & $123.1(7)$ \\
\hline C88 & O5 & $\mathrm{Co} 2$ & $116.3(6)$ & N2 & $\mathrm{C} 7$ & $\mathrm{C} 8$ & $116.9(7)$ \\
\hline C36 & N3 & Col & $115.3(6)$ & $\mathrm{C} 8$ & $\mathrm{C} 7$ & C6 & 120.0 \\
\hline C37 & N3 & Col & $126.0(9)$ & C74 & $\mathrm{C} 75$ & N7 & $122.3(7)$ \\
\hline C37 & N3 & C36 & $118.5(9)$ & C74 & $\mathrm{C} 75$ & C76 & 120.0 \\
\hline C89 & N6 & $\mathrm{Co} 2$ & $109.9(7)$ & C76 & $\mathrm{C} 75$ & N7 & $117.7(7)$ \\
\hline C81 & N6 & $\mathrm{Co} 2$ & $132.6(9)$ & C75 & $\mathrm{C} 74$ & C79 & 120.0 \\
\hline C81 & N6 & C89 & $117.4(11)$ & C75 & C74 & $\mathrm{C} 73$ & $115.9(8)$ \\
\hline C55 & N5 & $\mathrm{Co} 2$ & $113.5(6)$ & C79 & $\mathrm{C} 74$ & $\mathrm{C} 73$ & $124.1(8)$ \\
\hline C54 & N5 & $\mathrm{Co} 2$ & $127.0(8)$ & $\mathrm{C} 74$ & C79 & $\mathrm{Cl} 7$ & $121.4(5)$ \\
\hline C54 & N5 & C55 & $119.3(9)$ & $\mathrm{C} 78$ & C79 & $\mathrm{Cl} 7$ & $118.6(5)$ \\
\hline $\mathrm{C} 8$ & $\mathrm{O} 3$ & Col & $114.6(6)$ & C78 & C79 & $\mathrm{C} 74$ & 120.0 \\
\hline C18 & $\mathrm{O} 2$ & Co1 & $114.4(8)$ & C79 & $\mathrm{C} 78$ & $\mathrm{C} 77$ & 120.0 \\
\hline C71 & N7 & $\mathrm{Co} 2$ & $132.8(10)$ & C78 & C77 & $\mathrm{Cl} 8$ & $117.8(5)$ \\
\hline C71 & N7 & C75 & $119.4(11)$ & $\mathrm{C} 78$ & C77 & $\mathrm{C} 76$ & 120.0 \\
\hline $\mathrm{C} 75$ & N7 & $\mathrm{Co} 2$ & $107.4(7)$ & C76 & C77 & $\mathrm{Cl} 8$ & $121.9(5)$ \\
\hline $\mathrm{C} 7$ & $\mathrm{~N} 2$ & Col & $108.4(7)$ & $\mathrm{O} 4$ & $\mathrm{C} 76$ & $\mathrm{C} 75$ & $119.9(6)$ \\
\hline
\end{tabular}




\begin{tabular}{|c|c|c|c|c|c|c|c|}
\hline $\mathrm{C} 3$ & $\mathrm{~N} 2$ & Co1 & $131.7(9)$ & $\mathrm{O} 4$ & $\mathrm{C} 76$ & C77 & $120.1(6)$ \\
\hline $\mathrm{C} 3$ & $\mathrm{~N} 2$ & $\mathrm{C} 7$ & $119.6(11)$ & C77 & C76 & C75 & 120.0 \\
\hline C60 & N8 & $\mathrm{Co} 2$ & $113.1(7)$ & $\mathrm{C} 13$ & N1 & Col & $133.5(15)$ \\
\hline C61 & N8 & $\mathrm{Co} 2$ & $127.7(8)$ & $\mathrm{C} 13$ & N1 & $\mathrm{C} 17$ & $118.2(15)$ \\
\hline C61 & N8 & C60 & $119.2(10)$ & $\mathrm{C} 17$ & N1 & Col & $107.7(9)$ \\
\hline C76 & $\mathrm{O} 4$ & $\mathrm{Co} 2$ & $116.1(6)$ & C39 & $\mathrm{C} 38$ & C37 & $120.9(12)$ \\
\hline C56 & C52 & C51 & $124.2(8)$ & N2 & $\mathrm{C} 3$ & $\mathrm{C} 2$ & $121.5(12)$ \\
\hline C53 & C52 & C56 & $115.9(9)$ & $\mathrm{N} 2$ & $\mathrm{C} 3$ & $\mathrm{C} 4$ & $120.4(12)$ \\
\hline C53 & C52 & C51 & $119.9(10)$ & $\mathrm{C} 4$ & $\mathrm{C} 3$ & $\mathrm{C} 2$ & $118.1(12)$ \\
\hline $\mathrm{C} 26$ & N4 & Co1 & $113.4(6)$ & C68 & C69 & C64 & 120.0 \\
\hline $\mathrm{C} 22$ & N4 & Co1 & $128.5(8)$ & C67 & C68 & C69 & 120.0 \\
\hline $\mathrm{C} 22$ & N4 & $\mathrm{C} 26$ & $118.1(9)$ & C68 & C67 & C66 & 120.0 \\
\hline C35 & C34 & C33 & 120.0 & C67 & C66 & C65 & 120.0 \\
\hline C34 & $\mathrm{C} 35$ & C39 & $121.7(6)$ & C64 & C65 & C66 & 120.0 \\
\hline $\mathrm{C} 36$ & $\mathrm{C} 35$ & C34 & 120.0 & C69 & C64 & C63 & $121.9(7)$ \\
\hline C36 & $\mathrm{C} 35$ & C39 & $117.7(6)$ & C65 & C64 & C63 & $118.1(8)$ \\
\hline N3 & $\mathrm{C} 36$ & C35 & $123.0(6)$ & C65 & C64 & C69 & 120.0 \\
\hline N3 & $\mathrm{C} 36$ & $\mathrm{C} 26$ & $116.9(6)$ & $\mathrm{C} 73$ & $\mathrm{C} 72$ & C71 & $122.9(14)$ \\
\hline $\mathrm{C} 35$ & $\mathrm{C} 36$ & $\mathrm{C} 26$ & 120.0 & N8 & C61 & C62 & $122.2(12)$ \\
\hline N4 & $\mathrm{C} 26$ & C36 & $117.4(6)$ & N6 & C81 & $\mathrm{C} 82$ & $122.6(14)$ \\
\hline N4 & $\mathrm{C} 26$ & $\mathrm{C} 25$ & $122.6(6)$ & N6 & C81 & $\mathrm{C} 80$ & $117.7(15)$ \\
\hline $\mathrm{C} 25$ & $\mathrm{C} 26$ & C36 & 120.0 & $\mathrm{C} 82$ & C81 & $\mathrm{C} 80$ & $119.7(15)$ \\
\hline $\mathrm{C} 26$ & $\mathrm{C} 25$ & C33 & 120.0 & $\mathrm{C} 72$ & $\mathrm{C} 73$ & C74 & $118.6(13)$ \\
\hline $\mathrm{C} 26$ & $\mathrm{C} 25$ & $\mathrm{C} 24$ & $117.8(6)$ & $\mathrm{C} 44$ & $\mathrm{C} 45$ & $\mathrm{C} 40$ & 120.0 \\
\hline C33 & $\mathrm{C} 25$ & $\mathrm{C} 24$ & $121.7(6)$ & $\mathrm{C} 45$ & $\mathrm{C} 44$ & C43 & 120.0 \\
\hline $\mathrm{C} 25$ & C33 & C34 & 120.0 & $\mathrm{C} 42$ & $\mathrm{C} 43$ & C44 & 120.0 \\
\hline N4 & $\mathrm{C} 22$ & $\mathrm{C} 23$ & $123.9(11)$ & $\mathrm{C} 41$ & $\mathrm{C} 42$ & $\mathrm{C} 43$ & 120.0 \\
\hline C55 & C56 & C52 & $117.2(6)$ & $\mathrm{C} 42$ & $\mathrm{C} 41$ & $\mathrm{C} 40$ & 120.0 \\
\hline C55 & C56 & C57 & 120.0 & $\mathrm{C} 45$ & $\mathrm{C} 40$ & C39 & $118.9(7)$ \\
\hline $\mathrm{C} 57$ & C56 & C52 & $122.2(6)$ & $\mathrm{C} 41$ & $\mathrm{C} 40$ & C39 & $120.5(7)$ \\
\hline N5 & C55 & C56 & $122.5(6)$ & $\mathrm{C} 41$ & $\mathrm{C} 40$ & $\mathrm{C} 45$ & 120.0 \\
\hline N5 & C55 & C60 & $117.4(6)$ & C61 & C62 & C63 & $122.6(12)$ \\
\hline C56 & C55 & C60 & 120.0 & $\mathrm{C} 5$ & $\mathrm{C} 4$ & $\mathrm{C} 3$ & $120.2(12)$ \\
\hline N8 & C60 & C55 & $118.1(6)$ & $\mathrm{C} 82$ & $\mathrm{C} 83$ & C84 & $117.5(14)$ \\
\hline N8 & C60 & C59 & $121.7(6)$ & $\mathrm{C} 15$ & $\mathrm{C} 14$ & C13 & $112.6(19)$ \\
\hline C55 & C60 & C59 & 120.0 & $\mathrm{C} 46$ & $\mathrm{C} 47$ & $\mathrm{C} 48$ & 120.0 \\
\hline C60 & C59 & C63 & $117.0(6)$ & $\mathrm{C} 47$ & $\mathrm{C} 46$ & C51 & 120.0 \\
\hline C58 & C59 & C60 & 120.0 & $\mathrm{C} 46$ & $\mathrm{C} 51$ & C52 & $118.6(7)$ \\
\hline C58 & C59 & C63 & $122.4(6)$ & C50 & C51 & C52 & $121.3(7)$ \\
\hline C57 & C58 & C59 & 120.0 & C50 & C51 & $\mathrm{C} 46$ & 120.0 \\
\hline C58 & C57 & C56 & 120.0 & C49 & $\mathrm{C} 50$ & C51 & 120.0 \\
\hline C52 & C53 & C54 & $122.1(11)$ & C50 & $\mathrm{C} 49$ & $\mathrm{C} 48$ & 120.0 \\
\hline $\mathrm{O} 5$ & C88 & C89 & $119.0(6)$ & C49 & $\mathrm{C} 48$ & $\mathrm{C} 47$ & 120.0 \\
\hline $\mathrm{O} 5$ & C88 & C87 & $121.0(6)$ & C81 & $\mathrm{C} 82$ & C83 & $120.6(14)$ \\
\hline C89 & C88 & C87 & 120.0 & $\mathrm{C} 4$ & $\mathrm{C} 5$ & C6 & $118.5(12)$ \\
\hline
\end{tabular}




\begin{tabular}{cccccccc}
\hline $\mathrm{N} 6$ & $\mathrm{C} 89$ & $\mathrm{C} 88$ & $117.1(7)$ & $\mathrm{N} 1$ & $\mathrm{C} 13$ & $\mathrm{C} 14$ & $123(2)$ \\
$\mathrm{N} 6$ & $\mathrm{C} 89$ & $\mathrm{C} 84$ & $122.9(7)$ & $\mathrm{N} 1$ & $\mathrm{C} 13$ & $\mathrm{C} 12$ & $120.1(17)$ \\
$\mathrm{C} 88$ & $\mathrm{C} 89$ & $\mathrm{C} 84$ & 120.0 & $\mathrm{C} 12$ & $\mathrm{C} 13$ & $\mathrm{C} 14$ & $117.2(18)$ \\
$\mathrm{C} 89$ & $\mathrm{C} 84$ & $\mathrm{C} 83$ & $119.0(9)$ & $\mathrm{C} 20$ & $\mathrm{C} 21$ & $\mathrm{C} 16$ & 120.0 \\
$\mathrm{C} 85$ & $\mathrm{C} 84$ & $\mathrm{C} 89$ & 120.0 & $\mathrm{C} 20$ & $\mathrm{C} 21$ & $\mathrm{C} 13$ & $117.5(9)$ \\
$\mathrm{C} 85$ & $\mathrm{C} 84$ & $\mathrm{C} 83$ & $121.0(9)$ & $\mathrm{C} 16$ & $\mathrm{C} 21$ & $\mathrm{C} 13$ & $122.4(9)$ \\
$\mathrm{C} 84$ & $\mathrm{C} 85$ & $\mathrm{C} 16$ & $122.5(5)$ & $\mathrm{C} 19$ & $\mathrm{C} 20$ & $\mathrm{C} 21$ & 120.0 \\
$\mathrm{C} 84$ & $\mathrm{C} 85$ & $\mathrm{C} 86$ & 120.0 & $\mathrm{C} 20$ & $\mathrm{C} 19$ & $\mathrm{C} 14$ & $119.8(7)$ \\
$\mathrm{C} 86$ & $\mathrm{C} 85$ & $\mathrm{C} 16$ & $117.5(5)$ & $\mathrm{C} 20$ & $\mathrm{C} 19$ & $\mathrm{C} 18$ & 120.0 \\
$\mathrm{C} 85$ & $\mathrm{C} 86$ & $\mathrm{C} 87$ & 120.0 & $\mathrm{C} 18$ & $\mathrm{C} 19$ & $\mathrm{C} 14$ & $120.2(7)$ \\
$\mathrm{C} 88$ & $\mathrm{C} 87$ & $\mathrm{C} 15$ & $121.5(5)$ & $\mathrm{O} 2$ & $\mathrm{C} 18$ & $\mathrm{C} 19$ & $120.3(9)$ \\
$\mathrm{C} 86$ & $\mathrm{C} 87$ & $\mathrm{C} 15$ & $118.5(5)$ & $\mathrm{O} 2$ & $\mathrm{C} 18$ & $\mathrm{C} 17$ & $119.7(9)$ \\
$\mathrm{C} 86$ & $\mathrm{C} 87$ & $\mathrm{C} 88$ & 120.0 & $\mathrm{C} 17$ & $\mathrm{C} 18$ & $\mathrm{C} 19$ & 120.0 \\
$\mathrm{C} 59$ & $\mathrm{C} 63$ & $\mathrm{C} 64$ & $123.9(9)$ & $\mathrm{N} 1$ & $\mathrm{C} 17$ & $\mathrm{C} 18$ & $117.1(9)$ \\
$\mathrm{C} 62$ & $\mathrm{C} 63$ & $\mathrm{C} 59$ & $115.9(10)$ & $\mathrm{N} 1$ & $\mathrm{C} 17$ & $\mathrm{C} 16$ & $122.9(9)$ \\
$\mathrm{C} 62$ & $\mathrm{C} 63$ & $\mathrm{C} 64$ & $120.2(10)$ & $\mathrm{C} 18$ & $\mathrm{C} 17$ & $\mathrm{C} 16$ & 120.0 \\
$\mathrm{~N} 5$ & $\mathrm{C} 54$ & $\mathrm{C} 53$ & $122.2(11)$ & $\mathrm{C} 17$ & $\mathrm{C} 16$ & $\mathrm{C} 21$ & 120.0 \\
$\mathrm{C} 35$ & $\mathrm{C} 39$ & $\mathrm{C} 40$ & $124.2(10)$ & $\mathrm{C} 15$ & $\mathrm{C} 16$ & $\mathrm{C} 21$ & $120.7(13)$ \\
$\mathrm{C} 38$ & $\mathrm{C} 39$ & $\mathrm{C} 35$ & $116.7(10)$ & $\mathrm{C} 15$ & $\mathrm{C} 16$ & $\mathrm{C} 17$ & $119.3(13)$ \\
$\mathrm{C} 38$ & $\mathrm{C} 39$ & $\mathrm{C} 40$ & $119.1(11)$ & $\mathrm{C} 16$ & $\mathrm{C} 15$ & $\mathrm{C} 14$ & $124.3(19)$ \\
\hline
\end{tabular}

Table S31. Crystal data and structure refinement details for Co11.

\begin{tabular}{ll}
\hline Empirical formula & $\mathrm{C}_{32} \mathrm{H}_{18} \mathrm{Cl}_{6} \mathrm{CoN}_{4} \mathrm{O}_{2}$ \\
Formula weight & 762.13 \\
Temperature/K & $293(2)$ \\
Crystal system & triclinic \\
Space group & $\mathrm{P}-1$ \\
$\mathrm{a} / \AA$ & $10.1331(5)$ \\
$\mathrm{b} / \AA$ & $12.9198(6)$ \\
$\mathrm{c} / \AA$ & $13.0618(6)$ \\
$\alpha /{ }^{\circ}$ & $84.2450(10)$ \\
$\beta /{ }^{\circ}$ & $71.9900(10)$ \\
$\gamma /{ }^{\circ}$ & $71.7910(10)$ \\
Volume/ $\AA^{3}$ & $1544.78(13)$ \\
$\mathrm{Z}$ & 2 \\
$\rho_{\text {calc }} / \mathrm{cm}^{3}$ & 1.638 \\
$\mu / \mathrm{mm}^{-1}$ & 1.114 \\
$\mathrm{~F}(000)$ & 766.0 \\
Crystal size/mm & $0.27 \times 0.21 \times 0.17$ \\
Radiation & $\mathrm{MoK} \alpha(\lambda=0.71073)$ \\
$2 \Theta$ range for data collection $/{ }^{\circ} 6.238$ to 55.016 \\
Index ranges & $-13 \leq \mathrm{h} \leq 13,-16 \leq \mathrm{k} \leq 16,-16 \leq 1 \leq 16$ \\
\hline
\end{tabular}




\begin{tabular}{ll}
\hline Reflections collected & 32534 \\
Independent reflections & $7077\left[\mathrm{R}_{\text {int }}=0.0421, \mathrm{R}_{\text {sigma }}=0.0409\right]$ \\
Data/restraints/parameters & $7077 / 0 / 408$ \\
Goodness-of-fit on $\mathrm{F}^{2}$ & 1.013 \\
Final $\mathrm{R}$ indexes [I $>=2 \sigma(\mathrm{I})]$ & $\mathrm{R}_{1}=0.0419, \mathrm{wR}_{2}=0.0916$ \\
Final R indexes [all data] & $\mathrm{R}_{1}=0.0695, \mathrm{wR}_{2}=0.1014$ \\
Largest diff. peak/hole $/ \mathrm{e} \AA^{-3} 0.40 /-0.41$ \\
\hline
\end{tabular}

Table S32. Selected bond lengths ( $\AA$ ) for Co11.

\begin{tabular}{|c|c|c|c|c|c|}
\hline \multicolumn{2}{|c|}{$\overline{\text { Atom Atom }}$} & \multirow{2}{*}{$\frac{\text { Length/§ }}{2.0264(17)}$} & \multicolumn{2}{|c|}{ Atom Atom } & \multirow{2}{*}{$\frac{\text { Length/Å }}{1.409(4)}$} \\
\hline $\mathrm{Col}$ & $\mathrm{O} 1$ & & $\overline{\mathrm{C} 10}$ & $\mathrm{C} 7$ & \\
\hline Col & $\mathrm{O} 2$ & $2.0138(17)$ & $\mathrm{C} 10$ & $\mathrm{C} 11$ & $1.349(4)$ \\
\hline Col & N1 & $2.139(2)$ & $\mathrm{C} 7$ & $\mathrm{C} 8$ & $1.426(4)$ \\
\hline Col & $\mathrm{N} 2$ & $2.162(2)$ & $\mathrm{C} 13$ & $\mathrm{C} 18$ & $1.441(3)$ \\
\hline Col & N4 & $2.204(2)$ & $\mathrm{C} 13$ & $\mathrm{C} 14$ & $1.395(3)$ \\
\hline Col & $\mathrm{N} 3$ & $2.223(2)$ & $\mathrm{C} 1$ & $\mathrm{C} 2$ & $1.383(4)$ \\
\hline $\mathrm{C} 15$ & $\mathrm{C} 30$ & $1.734(3)$ & $\mathrm{C} 18$ & $\mathrm{C} 17$ & $1.414(3)$ \\
\hline $\mathrm{Cl} 3$ & $\mathrm{C} 14$ & $1.734(3)$ & $\mathrm{C} 4$ & C9 & $1.425(4)$ \\
\hline $\mathrm{Cl} 2$ & $\mathrm{C} 10$ & $1.727(3)$ & $\mathrm{C} 4$ & $\mathrm{C} 3$ & $1.410(4)$ \\
\hline $\mathrm{Cl} 4$ & $\mathrm{C} 16$ & $1.746(3)$ & $\mathrm{C} 29$ & $\mathrm{C} 30$ & $1.386(3)$ \\
\hline Cl1 & $\mathrm{C} 3$ & $1.721(3)$ & $\mathrm{C} 14$ & $\mathrm{C} 15$ & $1.392(4)$ \\
\hline $\mathrm{Cl} 6$ & $\mathrm{C} 32$ & $1.742(3)$ & $\mathrm{C} 30$ & $\mathrm{C} 31$ & $1.391(4)$ \\
\hline $\mathrm{O} 1$ & $\mathrm{C} 13$ & $1.285(3)$ & $\mathrm{C} 31$ & $\mathrm{C} 32$ & $1.361(4)$ \\
\hline $\mathrm{O} 2$ & $\mathrm{C} 29$ & $1.291(3)$ & $\mathrm{C} 17$ & $\mathrm{C} 16$ & $1.401(4)$ \\
\hline N1 & $\mathrm{C} 5$ & $1.351(3)$ & $\mathrm{C} 17$ & $\mathrm{C} 19$ & $1.412(4)$ \\
\hline N1 & $\mathrm{C} 1$ & $1.324(3)$ & $\mathrm{C} 21$ & $\mathrm{C} 20$ & $1.405(4)$ \\
\hline N2 & C6 & $1.350(3)$ & $\mathrm{C} 21$ & $\mathrm{C} 22$ & $1.495(4)$ \\
\hline N2 & $\mathrm{C} 12$ & $1.320(3)$ & $\mathrm{C} 8$ & $\mathrm{C} 9$ & $1.348(4)$ \\
\hline N4 & $\mathrm{C} 28$ & $1.372(3)$ & $\mathrm{C} 11$ & $\mathrm{C} 12$ & $1.393(4)$ \\
\hline N4 & $\mathrm{C} 24$ & $1.324(3)$ & $\mathrm{C} 16$ & $\mathrm{C} 15$ & $1.360(4)$ \\
\hline N3 & $\mathrm{C} 18$ & $1.375(3)$ & $\mathrm{C} 27$ & $\mathrm{C} 32$ & $1.399(4)$ \\
\hline N3 & $\mathrm{C} 21$ & $1.327(3)$ & $\mathrm{C} 27$ & $\mathrm{C} 26$ & $1.405(4)$ \\
\hline C6 & $\mathrm{C} 5$ & $1.445(3)$ & $\mathrm{C} 3$ & $\mathrm{C} 2$ & $1.353(4)$ \\
\hline C6 & $\mathrm{C} 7$ & $1.409(3)$ & C19 & $\mathrm{C} 20$ & $1.347(4)$ \\
\hline $\mathrm{C} 5$ & $\mathrm{C} 4$ & $1.401(3)$ & $\mathrm{C} 24$ & $\mathrm{C} 23$ & $1.490(4)$ \\
\hline $\mathrm{C} 28$ & $\mathrm{C} 29$ & $1.439(3)$ & $\mathrm{C} 24$ & $\mathrm{C} 25$ & $1.403(4)$ \\
\hline $\mathrm{C} 28$ & $\mathrm{C} 27$ & $1.419(3)$ & $\mathrm{C} 26$ & $\mathrm{C} 25$ & $1.351(4)$ \\
\hline
\end{tabular}

Table S33. Selected bond angles $\left(^{\circ}\right)$ for Co11.

\begin{tabular}{llll}
\hline Atom Atom Atom & Angle $/^{\circ}$ & Atom Atom Atom & Angle ${ }^{\circ}$ \\
\hline
\end{tabular}




\begin{tabular}{|c|c|c|c|c|c|c|c|}
\hline $\mathrm{O} 1$ & Col & N1 & $86.89(7)$ & N3 & C18 & $\mathrm{C} 13$ & $115.6(2)$ \\
\hline $\mathrm{O} 1$ & Co1 & N2 & $86.78(7)$ & N3 & C18 & $\mathrm{C} 17$ & $122.3(2)$ \\
\hline $\mathrm{O} 1$ & Col & N4 & $105.79(7)$ & $\mathrm{C} 17$ & $\mathrm{C} 18$ & $\mathrm{C} 13$ & $122.1(2)$ \\
\hline $\mathrm{O} 1$ & Col & N3 & $78.45(7)$ & $\mathrm{C} 5$ & $\mathrm{C} 4$ & C9 & $119.5(2)$ \\
\hline $\mathrm{O} 2$ & Co1 & $\mathrm{O} 1$ & $174.33(7)$ & $\mathrm{C} 5$ & $\mathrm{C} 4$ & $\mathrm{C} 3$ & $115.8(2)$ \\
\hline $\mathrm{O} 2$ & Col & N1 & $89.91(7)$ & $\mathrm{C} 3$ & $\mathrm{C} 4$ & $\mathrm{C} 9$ & $124.8(2)$ \\
\hline $\mathrm{O} 2$ & Co1 & N2 & $87.94(7)$ & $\mathrm{O} 2$ & C29 & $\mathrm{C} 28$ & $120.3(2)$ \\
\hline $\mathrm{O} 2$ & Col & N4 & $79.11(7)$ & $\mathrm{O} 2$ & C29 & $\mathrm{C} 30$ & $124.3(2)$ \\
\hline $\mathrm{O} 2$ & $\mathrm{Co} 1$ & N3 & $104.14(7)$ & $\mathrm{C} 30$ & C29 & $\mathrm{C} 28$ & $115.4(2)$ \\
\hline N1 & Co1 & N2 & $77.15(8)$ & $\mathrm{C} 13$ & $\mathrm{C} 14$ & $\mathrm{Cl3}$ & $118.1(2)$ \\
\hline $\mathrm{N} 1$ & Co1 & N4 & $94.26(7)$ & $\mathrm{C} 15$ & C14 & $\mathrm{Cl} 3$ & $118.9(2)$ \\
\hline N1 & Col & N3 & $163.97(7)$ & $\mathrm{C} 15$ & $\mathrm{C} 14$ & $\mathrm{C} 13$ & $123.0(3)$ \\
\hline $\mathrm{N} 2$ & Col & N4 & $164.53(7)$ & $\mathrm{C} 29$ & C30 & $\mathrm{Cl} 5$ & $118.5(2)$ \\
\hline $\mathrm{N} 2$ & Col & N3 & $95.40(7)$ & $\mathrm{C} 29$ & C30 & C31 & $123.3(2)$ \\
\hline N4 & Col & N3 & $95.94(7)$ & C31 & C30 & $\mathrm{C} 15$ & $118.2(2)$ \\
\hline $\mathrm{C} 13$ & $\mathrm{O} 1$ & Co1 & $115.90(15)$ & C32 & C31 & $\mathrm{C} 30$ & $120.0(2)$ \\
\hline $\mathrm{C} 29$ & $\mathrm{O} 2$ & Col & $115.78(15)$ & $\mathrm{C} 16$ & C17 & $\mathrm{C} 18$ & $118.1(2)$ \\
\hline $\mathrm{C} 5$ & N1 & Co1 & $114.62(15)$ & $\mathrm{C} 16$ & $\mathrm{C} 17$ & C19 & $125.3(3)$ \\
\hline $\mathrm{C} 1$ & N1 & Co1 & $127.34(18)$ & C19 & $\mathrm{C} 17$ & $\mathrm{C} 18$ & $116.6(2)$ \\
\hline $\mathrm{C} 1$ & N1 & $\mathrm{C} 5$ & $118.0(2)$ & N3 & $\mathrm{C} 21$ & $\mathrm{C} 20$ & $121.2(3)$ \\
\hline C6 & N2 & Col & $113.67(15)$ & N3 & $\mathrm{C} 21$ & $\mathrm{C} 22$ & $118.7(2)$ \\
\hline $\mathrm{C} 12$ & N2 & Co1 & $128.56(18)$ & $\mathrm{C} 20$ & $\mathrm{C} 21$ & $\mathrm{C} 22$ & $120.1(2)$ \\
\hline $\mathrm{C} 12$ & N2 & C6 & $117.7(2)$ & C9 & $\mathrm{C} 8$ & $\mathrm{C} 7$ & $120.9(2)$ \\
\hline $\mathrm{C} 28$ & N4 & Co1 & $108.83(15)$ & $\mathrm{C} 10$ & C11 & $\mathrm{C} 12$ & $118.5(2)$ \\
\hline $\mathrm{C} 24$ & N4 & Col & $132.04(18)$ & $\mathrm{C} 17$ & C16 & $\mathrm{Cl} 4$ & $119.7(2)$ \\
\hline $\mathrm{C} 24$ & N4 & C28 & $119.1(2)$ & $\mathrm{C} 15$ & $\mathrm{C} 16$ & $\mathrm{Cl} 4$ & $119.1(2)$ \\
\hline $\mathrm{C} 18$ & N3 & Co1 & $108.86(15)$ & $\mathrm{C} 15$ & $\mathrm{C} 16$ & $\mathrm{C} 17$ & $121.1(2)$ \\
\hline $\mathrm{C} 21$ & N3 & Col & $132.09(18)$ & $\mathrm{C} 16$ & $\mathrm{C} 15$ & $\mathrm{C} 14$ & $120.4(3)$ \\
\hline $\mathrm{C} 21$ & N3 & $\mathrm{C} 18$ & $118.9(2)$ & $\mathrm{C} 32$ & $\mathrm{C} 27$ & $\mathrm{C} 28$ & $117.8(2)$ \\
\hline $\mathrm{N} 2$ & C6 & $\mathrm{C} 5$ & $117.4(2)$ & $\mathrm{C} 32$ & $\mathrm{C} 27$ & $\mathrm{C} 26$ & $125.6(2)$ \\
\hline $\mathrm{N} 2$ & C6 & $\mathrm{C} 7$ & $123.7(2)$ & $\mathrm{C} 26$ & $\mathrm{C} 27$ & $\mathrm{C} 28$ & $116.6(2)$ \\
\hline $\mathrm{C} 7$ & C6 & $\mathrm{C} 5$ & $118.9(2)$ & $\mathrm{C} 8$ & $\mathrm{C} 9$ & $\mathrm{C} 4$ & $121.3(3)$ \\
\hline $\mathrm{N} 1$ & $\mathrm{C} 5$ & C6 & $117.0(2)$ & N2 & $\mathrm{C} 12$ & $\mathrm{C} 11$ & $123.4(3)$ \\
\hline $\mathrm{N} 1$ & $\mathrm{C} 5$ & $\mathrm{C} 4$ & $123.2(2)$ & $\mathrm{C} 4$ & $\mathrm{C} 3$ & $\mathrm{Cl1}$ & $119.6(2)$ \\
\hline $\mathrm{C} 4$ & $\mathrm{C} 5$ & C6 & $119.7(2)$ & $\mathrm{C} 2$ & $\mathrm{C} 3$ & $\mathrm{Cl1}$ & $119.3(2)$ \\
\hline N4 & $\mathrm{C} 28$ & C29 & $115.8(2)$ & $\mathrm{C} 2$ & $\mathrm{C} 3$ & $\mathrm{C} 4$ & $121.2(2)$ \\
\hline N4 & $\mathrm{C} 28$ & $\mathrm{C} 27$ & $122.2(2)$ & C31 & C32 & $\mathrm{Cl} 6$ & $118.9(2)$ \\
\hline $\mathrm{C} 27$ & $\mathrm{C} 28$ & C29 & $122.0(2)$ & C31 & C32 & $\mathrm{C} 27$ & $121.4(2)$ \\
\hline $\mathrm{C} 7$ & $\mathrm{C} 10$ & $\mathrm{Cl} 2$ & $119.0(2)$ & $\mathrm{C} 27$ & C32 & $\mathrm{Cl} 6$ & $119.7(2)$ \\
\hline $\mathrm{C} 11$ & $\mathrm{C} 10$ & $\mathrm{Cl} 2$ & $119.8(2)$ & $\mathrm{C} 20$ & C19 & $\mathrm{C} 17$ & $120.0(3)$ \\
\hline C11 & $\mathrm{C} 10$ & $\mathrm{C} 7$ & $121.3(2)$ & C19 & $\mathrm{C} 20$ & $\mathrm{C} 21$ & $120.9(3)$ \\
\hline C6 & $\mathrm{C} 7$ & $\mathrm{C} 10$ & $115.4(2)$ & $\mathrm{C} 3$ & $\mathrm{C} 2$ & $\mathrm{C} 1$ & $118.4(2)$ \\
\hline C6 & $\mathrm{C} 7$ & C8 & $119.8(2)$ & N4 & C24 & $\mathrm{C} 23$ & $119.2(2)$ \\
\hline $\mathrm{C} 10$ & $\mathrm{C} 7$ & $\mathrm{C} 8$ & $124.8(2)$ & N4 & C24 & $\mathrm{C} 25$ & $121.1(3)$ \\
\hline $\mathrm{O} 1$ & $\mathrm{C} 13$ & $\mathrm{C} 18$ & $120.6(2)$ & $\mathrm{C} 25$ & C24 & $\mathrm{C} 23$ & $119.6(3)$ \\
\hline
\end{tabular}




\begin{tabular}{|c|c|c|c|c|c|c|c|}
\hline O1 & $\mathrm{C} 13$ & $\mathrm{C} 14$ & $124.1(2)$ & $\mathrm{C} 25$ & $\mathrm{C} 26$ & $\mathrm{C} 27$ & $120.0(3)$ \\
\hline C14 & $\mathrm{C} 13$ & C18 & $115.3(2)$ & $\mathrm{C} 26$ & $\mathrm{C} 25$ & $\mathrm{C} 24$ & $120.9(3)$ \\
\hline N1 & $\mathrm{C} 1$ & $\mathrm{C} 2$ & $123.4(3)$ & & & & \\
\hline
\end{tabular}

Table S34. Crystal data and structure refinement details for Co12.

\begin{tabular}{ll}
\hline Empirical formula & $\mathrm{C}_{30} \mathrm{H}_{20} \mathrm{Cl}_{2} \mathrm{CoI}_{2} \mathrm{~N}_{4} \mathrm{O}_{2}$ \\
Formula weight & 852.13 \\
Temperature/K & $293(2)$ \\
Crystal system & monoclinic \\
Space group & $\mathrm{C} 2 / \mathrm{c}$ \\
$\mathrm{a} / \AA$ & $22.2551(9)$ \\
$\mathrm{b} / \AA$ & $11.6121(5)$ \\
$\mathrm{c} / \AA$ & $16.1476(6)$ \\
$\alpha /{ }^{\circ}$ & 90 \\
$\beta /{ }^{\circ}$ & $119.1630(10)$ \\
$\gamma^{\circ}$ & 90 \\
Volume/ $/ \AA^{3}$ & $3644.0(3)$ \\
$\mathrm{Z}$ & 4 \\
$\rho_{\text {calc }} / \mathrm{cm}^{3}$ & 1.553 \\
$\mu / \mathrm{mm}^{-1}$ & 2.341 \\
$\mathrm{~F}(000)$ & 1644.0 \\
Crystal size/mm & \\
Radiation & $0.26 \times 0.21 \times 0.13$ \\
$2 \Theta$ range for data collection/ & \multicolumn{1}{c}{6.188 to 55.112} \\
Index ranges & $-28 \leq \mathrm{h} \leq 28,-15 \leq \mathrm{k} \leq 15,-20 \leq 1 \leq 21$ \\
Reflections collected & 27940 \\
Independent reflections & $4197\left[\mathrm{R}_{\text {int }}=0.0378, \mathrm{R}_{\text {sigma }}=0.0294\right]$ \\
Data/restraints/parameters & $4197 / 42 / 187$ \\
Goodness-of-fit on $\mathrm{F}^{2}$ & 1.052 \\
Final R indexes $[\mathrm{I}>=2 \sigma(\mathrm{I})]$ & $\mathrm{R}_{1}=0.0488, \mathrm{wR}_{2}=0.1148$ \\
Final R indexes [all data] & $\mathrm{R}_{1}=0.0705, \mathrm{wR}_{2}=0.1234$ \\
Largest diff. peak/hole $/ \mathrm{e} \AA^{-3} 2.15 /-1.90$ \\
\hline
\end{tabular}

Table S35. Selected bond lengths $(\AA)$ for Co12.

\begin{tabular}{|c|c|c|c|c|c|c|c|}
\hline \multicolumn{3}{|c|}{ Atom Atom Atom } & \multirow{2}{*}{$\begin{array}{c}\text { Angle }^{\circ} \\
87.57(19)\end{array}$} & \multicolumn{3}{|c|}{ Atom Atom Atom } & \multirow{2}{*}{$\frac{\text { Angle }^{\circ}}{119.5(3)}$} \\
\hline $\mathrm{O} 1^{1}$ & $\mathrm{Co} 1$ & $\mathrm{O} 1$ & & C6 & $\mathrm{C} 7$ & I1 & \\
\hline $\mathrm{O} 1$ & Col & $\mathrm{N} 1$ & $86.14(14)$ & C6 & C7 & $\mathrm{C} 8$ & $120.8(5)$ \\
\hline $\mathrm{O} 1$ & $\mathrm{Co} 1$ & $\mathrm{~N} 1^{1}$ & $88.81(13)$ & $\mathrm{C} 8$ & $\mathrm{C} 7$ & I1 & $119.7(4)$ \\
\hline $\mathrm{O} 1^{1}$ & Co1 & $\mathrm{N} 1$ & $88.82(13)$ & N1 & $\mathrm{C} 5$ & C6 & $115.9(4)$ \\
\hline $\mathrm{O} 1^{1}$ & Col & $\mathrm{N} 1^{1}$ & $86.14(14)$ & N1 & $\mathrm{C} 5$ & $\mathrm{C} 4$ & $121.6(4)$ \\
\hline $\mathrm{O} 1$ & Co1 & $\mathrm{N} 2^{1}$ & $176.21(15)$ & C6 & $\mathrm{C} 5$ & $\mathrm{C} 4$ & $122.5(4)$ \\
\hline
\end{tabular}




\begin{tabular}{cccccccc}
\hline $\mathrm{O} 1$ & $\mathrm{C} 1$ & $\mathrm{~N} 2$ & $95.03(15)$ & $\mathrm{C} 9$ & $\mathrm{C} 8$ & $\mathrm{C} 7$ & $120.4(5)$ \\
$\mathrm{O}^{1}$ & $\mathrm{C} 1$ & $\mathrm{~N} 2$ & $176.22(15)$ & $\mathrm{N} 2$ & $\mathrm{C} 14$ & $\mathrm{C} 13$ & $122.4(6)$ \\
$\mathrm{O}^{1}$ & $\mathrm{C} 1$ & $\mathrm{~N} 21$ & $95.03(15)$ & $\mathrm{C} 9$ & $\mathrm{C} 4$ & $\mathrm{C} 5$ & $115.7(4)$ \\
$\mathrm{N} 1$ & $\mathrm{C} 1$ & $\mathrm{~N}^{1}$ & $173.0(2)$ & $\mathrm{C} 3$ & $\mathrm{C} 4$ & $\mathrm{C} 5$ & $117.0(4)$ \\
$\mathrm{N} 1{ }^{1}$ & $\mathrm{C} 1$ & $\mathrm{~N} 21$ & $88.62(15)$ & $\mathrm{C} 3$ & $\mathrm{C} 4$ & $\mathrm{C} 9$ & $127.2(4)$ \\
$\mathrm{N} 1{ }^{1}$ & $\mathrm{C} 1$ & $\mathrm{~N} 2$ & $96.65(16)$ & $\mathrm{N} 1$ & $\mathrm{C} 1$ & $\mathrm{C} 2$ & $121.0(5)$ \\
$\mathrm{N} 1$ & $\mathrm{C} 1$ & $\mathrm{~N} 2$ & $88.62(15)$ & $\mathrm{C} 8$ & $\mathrm{C} 9$ & $\mathrm{C} 11$ & $119.1(4)$ \\
$\mathrm{N} 1$ & $\mathrm{C} 1$ & $\mathrm{~N} 21$ & $96.65(16)$ & $\mathrm{C} 8$ & $\mathrm{C} 9$ & $\mathrm{C} 4$ & $122.5(4)$ \\
$\mathrm{N} 21$ & $\mathrm{C} 1$ & $\mathrm{~N} 2$ & $82.5(2)$ & $\mathrm{C} 4$ & $\mathrm{C} 9$ & $\mathrm{C} 11$ & $118.4(4)$ \\
$\mathrm{C} 6$ & $\mathrm{O} 1$ & $\mathrm{C} 1$ & $110.6(3)$ & $\mathrm{C} 2$ & $\mathrm{C} 3$ & $\mathrm{C} 4$ & $119.6(4)$ \\
$\mathrm{C} 5$ & $\mathrm{~N} 1$ & $\mathrm{C} 1$ & $109.4(3)$ & $\mathrm{C} 3$ & $\mathrm{C} 2$ & $\mathrm{C} 1$ & $120.6(5)$ \\
$\mathrm{C} 1$ & $\mathrm{~N} 1$ & $\mathrm{C} 1$ & $130.4(3)$ & $\mathrm{C} 12$ & $\mathrm{C} 11$ & $\mathrm{C} 10$ & $119.5(6)$ \\
$\mathrm{C} 1$ & $\mathrm{~N} 1$ & $\mathrm{C} 5$ & $120.2(4)$ & $\mathrm{N} 2$ & $\mathrm{C} 10$ & $\mathrm{C} 11$ & $120.2(6)$ \\
$\mathrm{O} 1$ & $\mathrm{C} 6$ & $\mathrm{C} 7$ & $125.1(4)$ & $\mathrm{N} 2$ & $\mathrm{C} 10$ & $\mathrm{C} 10^{1}$ & $113.7(3)$ \\
$\mathrm{O} 1$ & $\mathrm{C} 6$ & $\mathrm{C} 5$ & $117.1(4)$ & $\mathrm{C} 11$ & $\mathrm{C} 10$ & $\mathrm{C} 10^{1}$ & $126.1(4)$ \\
$\mathrm{C} 7$ & $\mathrm{C} 6$ & $\mathrm{C} 5$ & $117.7(4)$ & $\mathrm{C} 14$ & $\mathrm{C} 13$ & $\mathrm{C} 15$ & $121.1(6)$ \\
$\mathrm{C} 14$ & $\mathrm{~N} 2$ & $\mathrm{C} 1$ & $124.5(3)$ & $\mathrm{C} 12$ & $\mathrm{C} 13$ & $\mathrm{C} 14$ & $116.1(6)$ \\
$\mathrm{C} 14$ & $\mathrm{~N} 2$ & $\mathrm{C} 10$ & $120.3(4)$ & $\mathrm{C} 12$ & $\mathrm{C} 13$ & $\mathrm{C} 15$ & $122.7(6)$ \\
$\mathrm{C} 10$ & $\mathrm{~N} 2$ & $\mathrm{C} 1$ & $114.9(3)$ & $\mathrm{C} 11$ & $\mathrm{C} 12$ & $\mathrm{C} 13$ & $121.5(6)$ \\
\hline
\end{tabular}

${ }^{1} 1-X,+Y, 1 / 2-Z$

Table S36. Selected bond angles $\left({ }^{\circ}\right)$ for Co12.

\begin{tabular}{|c|c|c|c|c|c|c|c|c|c|}
\hline $\mathbf{A}$ & B & $\mathrm{C}$ & D & Angle $/^{\circ}$ & $\mathbf{A}$ & B & $\mathrm{C}$ & D & Angle $/^{\circ}$ \\
\hline I1 & $\mathrm{C} 7$ & $\mathrm{C} 8$ & C9 & $179.7(4)$ & $\mathrm{C} 7$ & C6 & $\mathrm{C} 5$ & N1 & $175.0(4)$ \\
\hline Co1 & $\mathrm{O} 1$ & C6 & $\mathrm{C} 7$ & $-171.1(4)$ & $\mathrm{C} 7$ & C6 & $\mathrm{C} 5$ & $\mathrm{C} 4$ & $-4.9(6)$ \\
\hline Co1 & $\mathrm{O} 1$ & C6 & C5 & $9.6(5)$ & C7 & $\mathrm{C} 8$ & C9 & $\mathrm{Cl1}$ & $177.6(5)$ \\
\hline Co1 & N1 & $\mathrm{C} 5$ & C6 & $-1.4(4)$ & $\mathrm{C} 7$ & $\mathrm{C} 8$ & C9 & $\mathrm{C} 4$ & $-2.2(9)$ \\
\hline Co1 & N1 & $\mathrm{C} 5$ & $\mathrm{C} 4$ & $178.5(3)$ & $\mathrm{C} 5$ & N1 & $\mathrm{C} 1$ & $\mathrm{C} 2$ & $0.9(7)$ \\
\hline Col & N1 & $\mathrm{C} 1$ & $\mathrm{C} 2$ & $-179.3(3)$ & C5 & C6 & $\mathrm{C} 7$ & I1 & $-176.3(3)$ \\
\hline Co1 & $\mathrm{N} 2$ & C14 & C13 & $174.3(4)$ & $\mathrm{C} 5$ & $\mathrm{C} 6$ & $\mathrm{C} 7$ & $\mathrm{C} 8$ & $4.6(7)$ \\
\hline Co1 & $\mathrm{N} 2$ & $\mathrm{C} 10$ & C11 & $-175.2(5)$ & $\mathrm{C} 5$ & $\mathrm{C} 4$ & C9 & $\mathrm{Cl1}$ & $-177.9(4)$ \\
\hline Co1 & $\mathrm{N} 2$ & $\mathrm{C} 10$ & $\mathrm{C} 10^{1}$ & $4.6(8)$ & $\mathrm{C} 5$ & $\mathrm{C} 4$ & C9 & $\mathrm{C} 8$ & $1.9(8)$ \\
\hline $\mathrm{O} 1^{1}$ & Col & $\mathrm{O} 1$ & C6 & $80.9(3)$ & $\mathrm{C} 5$ & $\mathrm{C} 4$ & $\mathrm{C} 3$ & $\mathrm{C} 2$ & $1.4(7)$ \\
\hline $\mathrm{O} 1$ & C6 & $\mathrm{C} 7$ & I1 & $4.3(7)$ & C14 & N2 & $\mathrm{C} 10$ & C11 & $-1.3(9)$ \\
\hline $\mathrm{O} 1$ & C6 & $\mathrm{C} 7$ & $\mathrm{C} 8$ & $-174.8(5)$ & $\mathrm{C} 14$ & $\mathrm{~N} 2$ & $\mathrm{C} 10$ & $\mathrm{C} 10^{1}$ & $178.5(6)$ \\
\hline $\mathrm{O} 1$ & C6 & $\mathrm{C} 5$ & N1 & $-5.6(6)$ & C14 & $\mathrm{C} 13$ & C12 & $\mathrm{C} 11$ & $-0.2(12)$ \\
\hline $\mathrm{O} 1$ & C6 & $\mathrm{C} 5$ & $\mathrm{C} 4$ & $174.5(4)$ & $\mathrm{C} 4$ & $\mathrm{C} 3$ & $\mathrm{C} 2$ & $\mathrm{C} 1$ & $-2.2(7)$ \\
\hline $\mathrm{N} 1^{1}$ & Col & $\mathrm{O} 1$ & C6 & 167.1(3) & $\mathrm{C} 1$ & N1 & $\mathrm{C} 5$ & C6 & $178.4(4)$ \\
\hline N1 & Col & $\mathrm{O} 1$ & C6 & $-8.1(3)$ & $\mathrm{C} 1$ & N1 & $\mathrm{C} 5$ & $\mathrm{C} 4$ & $-1.7(6)$ \\
\hline N1 & $\mathrm{C} 5$ & $\mathrm{C} 4$ & C9 & $-178.2(4)$ & C9 & $\mathrm{C} 4$ & $\mathrm{C} 3$ & $\mathrm{C} 2$ & $179.9(5)$ \\
\hline N1 & $\mathrm{C} 5$ & $\mathrm{C} 4$ & C3 & $0.5(6)$ & $\mathrm{C} 3$ & $\mathrm{C} 4$ & C9 & $\mathrm{Cl1}$ & $3.6(7)$ \\
\hline N1 & $\mathrm{C} 1$ & $\mathrm{C} 2$ & $\mathrm{C} 3$ & $1.0(8)$ & $\mathrm{C} 3$ & $\mathrm{C} 4$ & C9 & $\mathrm{C} 8$ & $-176.6(5)$ \\
\hline
\end{tabular}




\begin{tabular}{llllllc}
\hline C6 & C7 & C8 & C9 & $-1.2(9)$ & C10 N2 C14 C13 & $1.0(8)$ \\
C6 & C5 & C4 & C9 & $1.7(6)$ & C10 C11 C12 C13 & $-0.1(13)$ \\
C6 & C5 & C4 4 & C3 & $-179.6(4)$ & C15 C13 C12 C11 & $-178.2(8)$ \\
N2 & Co1 & O1 1 & C6 & $-96.4(3)$ & C12 C11 C10 N2 & $0.9(11)$ \\
N2 & C14 1413 & C15 & $177.8(6)$ & C12 C11 C10 C10 1 & $-179.0(8)$ \\
N2 & C14 C13 & C12 & $-0.3(9)$ & & & \\
\hline
\end{tabular}

Table S37. Crystal data and structure refinement details for Co13.

\begin{tabular}{|c|c|}
\hline Empirical formula & $\mathrm{C}_{32} \mathrm{H}_{24} \mathrm{Cl}_{4} \mathrm{CoN}_{4} \mathrm{O}_{2}$ \\
\hline Formula weight & 697.28 \\
\hline Temperature/K & 293(2) \\
\hline Crystal system & triclinic \\
\hline Space group & $\mathrm{P}-1$ \\
\hline $\mathrm{a} / \AA ̊$ & $8.9338(7)$ \\
\hline $\mathrm{b} / \AA$ & $11.4622(10)$ \\
\hline $\mathrm{c} / \AA ̊$ & $15.9754(13)$ \\
\hline$\alpha /^{\circ}$ & $96.757(3)$ \\
\hline$\beta /{ }^{\circ}$ & $101.337(3)$ \\
\hline$\gamma /{ }^{\circ}$ & $97.265(3)$ \\
\hline Volume $/ \AA^{3}$ & $1573.9(2)$ \\
\hline $\mathrm{Z}$ & 2 \\
\hline$\rho_{\text {calc }} \mathrm{g} / \mathrm{cm}^{3}$ & 1.471 \\
\hline$\mu / \mathrm{mm}^{-1}$ & 0.921 \\
\hline $\mathrm{F}(000)$ & 710.0 \\
\hline Crystal size $/ \mathrm{mm}^{3}$ & $0.25 \times 0.21 \times 0.16$ \\
\hline Radiation & $\operatorname{MoK} \alpha(\lambda=0.71073)$ \\
\hline \multicolumn{2}{|c|}{$2 \Theta$ range for data collection $/{ }^{\circ} 6.26$ to 55.142} \\
\hline Index ranges & $-11 \leq \mathrm{h} \leq 11,-14 \leq \mathrm{k} \leq 14,-20 \leq 1 \leq 20$ \\
\hline Reflections collected & 33819 \\
\hline Independent reflections & $7251\left[\mathrm{R}_{\mathrm{int}}=0.0664, \mathrm{R}_{\text {sigma }}=0.0756\right]$ \\
\hline Data/restraints/parameters & $7251 / 0 / 392$ \\
\hline Goodness-of-fit on $\mathrm{F}^{2}$ & 1.008 \\
\hline Final R indexes $[\mathrm{I}>=2 \sigma(\mathrm{I})]$ & $\mathrm{R}_{1}=0.0547, \mathrm{wR}_{2}=0.1081$ \\
\hline Final $\mathrm{R}$ indexes [all data] & $\mathrm{R}_{1}=0.1205, \mathrm{wR}_{2}=0.1283$ \\
\hline Largest diff. peak/hole / e $\AA$ & $0.93 /-0.89$ \\
\hline
\end{tabular}

Table S38. Selected bond lengths ( $\AA$ ) for Co13.

\begin{tabular}{|c|c|c|c|}
\hline Atom Atom & Length/Å & Atom Atom & Length/Å \\
\hline $\mathrm{Col}$ & $2.024(2)$ & C9 & $1.387(5)$ \\
\hline $\mathrm{Co} 1$ & $2.029(2)$ & $\mathrm{C} 26 \quad \mathrm{C} 25$ & $1.481(5)$ \\
\hline
\end{tabular}




\begin{tabular}{cccccc}
\hline Co1 & $\mathrm{N} 4$ & $2.146(3)$ & $\mathrm{C} 26$ & $\mathrm{C} 27$ & $1.386(5)$ \\
$\mathrm{C} 1$ & $\mathrm{~N} 3$ & $2.128(3)$ & $\mathrm{C} 16$ & $\mathrm{C} 15$ & $1.415(4)$ \\
$\mathrm{C} 11$ & $\mathrm{~N} 1$ & $2.232(3)$ & $\mathrm{C} 6$ & $\mathrm{C} 5$ & $1.404(4)$ \\
$\mathrm{C} 11$ & $\mathrm{~N} 2$ & $2.257(3)$ & $\mathrm{C} 2$ & $\mathrm{C} 3$ & $1.408(5)$ \\
$\mathrm{C} 11$ & $\mathrm{C} 8$ & $1.746(3)$ & $\mathrm{C} 2$ & $\mathrm{C} 1$ & $1.490(5)$ \\
$\mathrm{C} 13$ & $\mathrm{C} 18$ & $1.734(4)$ & $\mathrm{C} 15$ & $\mathrm{C} 14$ & $1.399(5)$ \\
$\mathrm{C} 14$ & $\mathrm{C} 20$ & $1.741(3)$ & $\mathrm{C} 5$ & $\mathrm{C} 4$ & $1.408(5)$ \\
$\mathrm{C} 12$ & $\mathrm{C} 10$ & $1.745(3)$ & $\mathrm{C} 5$ & $\mathrm{C} 10$ & $1.409(5)$ \\
$\mathrm{O} 2$ & $\mathrm{C} 17$ & $1.292(4)$ & $\mathrm{C} 4$ & $\mathrm{C} 3$ & $1.347(5)$ \\
$\mathrm{O} 1$ & $\mathrm{C} 7$ & $1.293(4)$ & $\mathrm{C} 23$ & $\mathrm{C} 22$ & $1.381(5)$ \\
$\mathrm{N} 4$ & $\mathrm{C} 26$ & $1.344(4)$ & $\mathrm{C} 23$ & $\mathrm{C} 24$ & $1.383(5)$ \\
$\mathrm{N} 4$ & $\mathrm{C} 30$ & $1.329(4)$ & $\mathrm{C} 23$ & $\mathrm{C} 31$ & $1.514(5)$ \\
$\mathrm{N} 3$ & $\mathrm{C} 25$ & $1.348(4)$ & $\mathrm{C} 12$ & $\mathrm{C} 13$ & $1.405(5)$ \\
$\mathrm{N} 3$ & $\mathrm{C} 21$ & $1.327(4)$ & $\mathrm{C} 12$ & $\mathrm{C} 11$ & $1.494(5)$ \\
$\mathrm{N} 1$ & $\mathrm{C} 6$ & $1.370(4)$ & $\mathrm{C} 10$ & $\mathrm{C} 9$ & $1.359(5)$ \\
$\mathrm{N} 1$ & $\mathrm{C} 2$ & $1.329(4)$ & $\mathrm{C} 19$ & $\mathrm{C} 18$ & $1.389(5)$ \\
$\mathrm{N} 2$ & $\mathrm{C} 16$ & $1.373(4)$ & $\mathrm{C} 25$ & $\mathrm{C} 24$ & $1.377(5)$ \\
$\mathrm{N} 2$ & $\mathrm{C} 12$ & $1.330(4)$ & $\mathrm{C} 22$ & $\mathrm{C} 21$ & $1.373(5)$ \\
$\mathrm{C} 7$ & $\mathrm{C} 8$ & $1.389(4)$ & $\mathrm{C} 14$ & $\mathrm{C} 13$ & $1.351(5)$ \\
$\mathrm{C} 7$ & $\mathrm{C} 6$ & $1.445(4)$ & $\mathrm{C} 27$ & $\mathrm{C} 28$ & $1.369(6)$ \\
$\mathrm{C} 20$ & $\mathrm{C} 15$ & $1.409(5)$ & $\mathrm{C} 30$ & $\mathrm{C} 29$ & $1.373(5)$ \\
$\mathrm{C} 20$ & $\mathrm{C} 19$ & $1.352(5)$ & $\mathrm{C} 28$ & $\mathrm{C} 32$ & $1.519(6)$ \\
$\mathrm{C} 17$ & $\mathrm{C} 16$ & $1.437(4)$ & $\mathrm{C} 28$ & $\mathrm{C} 29$ & $1.377(6)$ \\
$\mathrm{C} 17$ & $\mathrm{C} 18$ & $1.393(4)$ & & & \\
\hline
\end{tabular}

Table S39. Selected bond angles $\left({ }^{\circ}\right)$ for Co13.

\begin{tabular}{|c|c|c|c|c|c|c|c|}
\hline \multicolumn{3}{|c|}{ Atom Atom Atom } & \multirow{2}{*}{$\frac{\text { Angle }^{\circ}}{178.54(9)}$} & \multicolumn{3}{|c|}{ Atom Atom Atom } & \multirow{2}{*}{$\frac{\text { Angle }^{\circ}}{122.2(3)}$} \\
\hline $\mathrm{O} 2$ & Col & $\mathrm{O} 1$ & & $\mathrm{~N} 2$ & $\mathrm{C} 16$ & $\mathrm{C} 15$ & \\
\hline $\mathrm{O} 2$ & Co1 & N4 & $88.67(10)$ & $\mathrm{C} 15$ & $\mathrm{C} 16$ & $\mathrm{C} 17$ & $122.1(3)$ \\
\hline $\mathrm{O} 2$ & Col & N3 & $90.00(9)$ & N1 & C6 & $\mathrm{C} 7$ & $115.4(3)$ \\
\hline $\mathrm{O} 2$ & Co1 & N1 & $103.05(9)$ & N1 & C6 & $\mathrm{C} 5$ & $122.6(3)$ \\
\hline $\mathrm{O} 2$ & Co1 & $\mathrm{N} 2$ & $77.72(9)$ & C5 & C6 & $\mathrm{C} 7$ & $122.0(3)$ \\
\hline O1 & Co1 & N4 & $89.88(9)$ & N1 & $\mathrm{C} 2$ & $\mathrm{C} 3$ & $120.7(3)$ \\
\hline O1 & Col & N3 & $89.86(10)$ & N1 & $\mathrm{C} 2$ & $\mathrm{C} 1$ & $119.2(3)$ \\
\hline O1 & Col & N1 & $78.41(9)$ & $\mathrm{C} 3$ & $\mathrm{C} 2$ & $\mathrm{C} 1$ & $120.1(3)$ \\
\hline $\mathrm{O} 1$ & Col & $\mathrm{N} 2$ & $102.24(9)$ & $\mathrm{C} 20$ & $\mathrm{C} 15$ & $\mathrm{C} 16$ & $117.7(3)$ \\
\hline N4 & Co1 & N1 & $166.31(10)$ & $\mathrm{C} 14$ & $\mathrm{C} 15$ & $\mathrm{C} 20$ & $124.9(3)$ \\
\hline N4 & Col & N2 & $95.77(10)$ & $\mathrm{C} 14$ & $\mathrm{C} 15$ & $\mathrm{C} 16$ & $117.4(3)$ \\
\hline N3 & Co1 & N4 & $76.68(11)$ & C6 & $\mathrm{C} 5$ & $\mathrm{C} 4$ & $116.9(3)$ \\
\hline N3 & Co1 & N1 & $96.03(10)$ & C6 & $\mathrm{C} 5$ & $\mathrm{C} 10$ & $118.2(3)$ \\
\hline N3 & Col & N2 & $165.83(10)$ & C4 & C5 & $\mathrm{C} 10$ & $124.9(3)$ \\
\hline
\end{tabular}




\begin{tabular}{cccccccc}
\hline $\mathrm{N} 1$ & $\mathrm{C} 11$ & $\mathrm{~N} 2$ & $93.64(9)$ & $\mathrm{C} 3$ & $\mathrm{C} 4$ & $\mathrm{C} 5$ & $119.8(3)$ \\
$\mathrm{C} 17$ & $\mathrm{O} 2$ & $\mathrm{C} 11$ & $117.03(19)$ & $\mathrm{C} 4$ & $\mathrm{C} 3$ & $\mathrm{C} 2$ & $120.9(3)$ \\
$\mathrm{C} 7$ & $\mathrm{O} 1$ & $\mathrm{C} 1$ & $115.73(18)$ & $\mathrm{C} 22$ & $\mathrm{C} 23$ & $\mathrm{C} 24$ & $117.4(3)$ \\
$\mathrm{C} 26$ & $\mathrm{~N} 4$ & $\mathrm{C} 1$ & $115.4(2)$ & $\mathrm{C} 22$ & $\mathrm{C} 23$ & $\mathrm{C} 31$ & $122.1(4)$ \\
$\mathrm{C} 30$ & $\mathrm{~N} 4$ & $\mathrm{C} 1$ & $126.2(2)$ & $\mathrm{C} 24$ & $\mathrm{C} 23$ & $\mathrm{C} 31$ & $120.5(4)$ \\
$\mathrm{C} 30$ & $\mathrm{~N} 4$ & $\mathrm{C} 26$ & $118.2(3)$ & $\mathrm{N} 2$ & $\mathrm{C} 12$ & $\mathrm{C} 13$ & $121.0(3)$ \\
$\mathrm{C} 25$ & $\mathrm{~N} 3$ & $\mathrm{C} 1$ & $116.2(2)$ & $\mathrm{N} 2$ & $\mathrm{C} 12$ & $\mathrm{C} 11$ & $119.3(3)$ \\
$\mathrm{C} 21$ & $\mathrm{~N} 3$ & $\mathrm{C} 1$ & $126.0(2)$ & $\mathrm{C} 13$ & $\mathrm{C} 12$ & $\mathrm{C} 11$ & $119.7(3)$ \\
$\mathrm{C} 21$ & $\mathrm{~N} 3$ & $\mathrm{C} 25$ & $117.7(3)$ & $\mathrm{C} 5$ & $\mathrm{C} 10$ & $\mathrm{C} 12$ & $119.7(3)$ \\
$\mathrm{C} 6$ & $\mathrm{~N} 1$ & $\mathrm{C} 1$ & $109.12(19)$ & $\mathrm{C} 9$ & $\mathrm{C} 10$ & $\mathrm{C} 12$ & $118.9(3)$ \\
$\mathrm{C} 2$ & $\mathrm{~N} 1$ & $\mathrm{C} 1$ & $131.8(2)$ & $\mathrm{C} 9$ & $\mathrm{C} 10$ & $\mathrm{C} 5$ & $121.4(3)$ \\
$\mathrm{C} 2$ & $\mathrm{~N} 1$ & $\mathrm{C} 6$ & $119.0(3)$ & $\mathrm{C} 20$ & $\mathrm{C} 19$ & $\mathrm{C} 18$ & $120.1(3)$ \\
$\mathrm{C} 16$ & $\mathrm{~N} 2$ & $\mathrm{C} 1$ & $108.92(19)$ & $\mathrm{N} 3$ & $\mathrm{C} 25$ & $\mathrm{C} 26$ & $115.5(3)$ \\
$\mathrm{C} 12$ & $\mathrm{~N} 2$ & $\mathrm{C} 1$ & $132.4(2)$ & $\mathrm{N} 3$ & $\mathrm{C} 25$ & $\mathrm{C} 24$ & $121.7(3)$ \\
$\mathrm{C} 12$ & $\mathrm{~N} 2$ & $\mathrm{C} 16$ & $118.7(3)$ & $\mathrm{C} 24$ & $\mathrm{C} 25$ & $\mathrm{C} 26$ & $122.8(3)$ \\
$\mathrm{O} 1$ & $\mathrm{C} 7$ & $\mathrm{C} 8$ & $124.4(3)$ & $\mathrm{C} 21$ & $\mathrm{C} 22$ & $\mathrm{C} 23$ & $119.2(4)$ \\
$\mathrm{O} 1$ & $\mathrm{C} 7$ & $\mathrm{C} 6$ & $120.8(3)$ & $\mathrm{N} 3$ & $\mathrm{C} 21$ & $\mathrm{C} 22$ & $123.6(3)$ \\
$\mathrm{C} 8$ & $\mathrm{C} 7$ & $\mathrm{C} 6$ & $114.8(3)$ & $\mathrm{C} 10$ & $\mathrm{C} 9$ & $\mathrm{C} 8$ & $119.3(3)$ \\
$\mathrm{C} 15$ & $\mathrm{C} 20$ & $\mathrm{C} 14$ & $119.1(3)$ & $\mathrm{C} 17$ & $\mathrm{C} 18$ & $\mathrm{C} 13$ & $118.8(3)$ \\
$\mathrm{C} 19$ & $\mathrm{C} 20$ & $\mathrm{C} 14$ & $119.4(3)$ & $\mathrm{C} 19$ & $\mathrm{C} 18$ & $\mathrm{C} 13$ & $117.9(3)$ \\
$\mathrm{C} 19$ & $\mathrm{C} 20$ & $\mathrm{C} 15$ & $121.5(3)$ & $\mathrm{C} 19$ & $\mathrm{C} 18$ & $\mathrm{C} 17$ & $123.4(3)$ \\
$\mathrm{O} 2$ & $\mathrm{C} 17$ & $\mathrm{C} 16$ & $120.6(3)$ & $\mathrm{C} 25$ & $\mathrm{C} 24$ & $\mathrm{C} 23$ & $120.3(3)$ \\
$\mathrm{O} 2$ & $\mathrm{C} 17$ & $\mathrm{C} 18$ & $124.1(3)$ & $\mathrm{C} 13$ & $\mathrm{C} 14$ & $\mathrm{C} 15$ & $119.3(3)$ \\
$\mathrm{C} 18$ & $\mathrm{C} 17$ & $\mathrm{C} 16$ & $115.2(3)$ & $\mathrm{C} 14$ & $\mathrm{C} 13$ & $\mathrm{C} 12$ & $121.3(3)$ \\
$\mathrm{C} 7$ & $\mathrm{C} 8$ & $\mathrm{C} 11$ & $117.3(3)$ & $\mathrm{C} 28$ & $\mathrm{C} 27$ & $\mathrm{C} 26$ & $120.1(4)$ \\
$\mathrm{C} 9$ & $\mathrm{C} 8$ & $\mathrm{C} 11$ & $118.4(3)$ & $\mathrm{N} 4$ & $\mathrm{C} 30$ & $\mathrm{C} 29$ & $122.9(4)$ \\
$\mathrm{C} 9$ & $\mathrm{C} 8$ & $\mathrm{C} 7$ & $124.3(3)$ & $\mathrm{C} 27$ & $\mathrm{C} 28$ & $\mathrm{C} 32$ & $120.9(5)$ \\
$\mathrm{N} 4$ & $\mathrm{C} 26$ & $\mathrm{C} 25$ & $116.0(3)$ & $\mathrm{C} 27$ & $\mathrm{C} 28$ & $\mathrm{C} 29$ & $118.0(4)$ \\
$\mathrm{N} 4$ & $\mathrm{C} 26$ & $\mathrm{C} 27$ & $121.4(3)$ & $\mathrm{C} 29$ & $\mathrm{C} 28$ & $\mathrm{C} 32$ & $121.1(5)$ \\
$\mathrm{C} 27$ & $\mathrm{C} 26$ & $\mathrm{C} 25$ & $122.6(3)$ & $\mathrm{C} 30$ & $\mathrm{C} 29$ & $\mathrm{C} 28$ & $119.4(4)$ \\
$\mathrm{N} 2$ & $\mathrm{C} 16$ & $\mathrm{C} 17$ & $115.7(3)$ & & & & \\
\hline & & & & & & & \\
\hline
\end{tabular}

Table S40. Crystal data and structure refinement details for Co14.

\begin{tabular}{ll}
\hline Empirical formula & $\mathrm{C}_{30} \mathrm{H}_{16} \mathrm{CoI}_{4} \mathrm{~N}_{4} \mathrm{O}_{2}$ \\
Formula weight & 1031.00 \\
Temperature/K & $293(2)$ \\
Crystal system & monoclinic \\
Space group & $\mathrm{C} 2 / \mathrm{c}$ \\
$\mathrm{a} / \AA$ & $28.7855(19)$ \\
$\mathrm{b} / \AA$ & $16.3652(11)$ \\
$\mathrm{c} / \AA$ & $17.4765(11)$ \\
$\alpha /{ }^{\circ}$ & 90 \\
\hline
\end{tabular}




\begin{tabular}{ll}
\hline$\beta /{ }^{\circ}$ & $124.0550(10)$ \\
$\gamma /{ }^{\circ}$ & 90 \\
Volume $/ \AA^{3}$ & $6820.9(8)$ \\
$\mathrm{Z}$ & 8 \\
$\rho_{\text {calc }} / \mathrm{cm}^{3}$ & 2.008 \\
$\mu / \mathrm{mm}^{-1}$ & 4.155 \\
$\mathrm{~F}(000)$ & 3832.0 \\
Crystal size $/ \mathrm{mm}^{3}$ & $0.3 \times 0.2 \times 0.11$ \\
Radiation & $\mathrm{MoK} \alpha(\lambda=0.71073)$ \\
$2 \Theta$ range for data collection $/{ }^{\circ} 5.94$ to 55.142 \\
Index ranges & $-37 \leq \mathrm{h} \leq 37,-21 \leq \mathrm{k} \leq 21,-22 \leq 1 \leq 22$ \\
Reflections collected & 55519 \\
Independent reflections & $7881\left[\mathrm{R}_{\text {int }}=0.1151, \mathrm{R}_{\text {sigma }}=0.0952\right]$ \\
Data/restraints $/$ parameters & $7881 / 0 / 370$ \\
Goodness-of-fit on $\mathrm{F}^{2}$ & 1.026 \\
Final R indexes $[\mathrm{I}>=2 \sigma(\mathrm{I})]$ & $\mathrm{R}_{1}=0.0572, \mathrm{wR}_{2}=0.0931$ \\
Final R indexes [all data] & $\mathrm{R}_{1}=0.1539, \mathrm{wR}_{2}=0.1149$ \\
Largest diff. peak/hole / e $\AA^{-3} 0.90 /-0.90$ \\
\hline
\end{tabular}

Table S41. Selected bond lengths ( $\AA$ ) for Co14.

\begin{tabular}{|c|c|c|c|c|c|}
\hline \multicolumn{2}{|c|}{ Atom Atom } & \multirow{2}{*}{$\frac{\text { Length/Å }}{2.093(7)}$} & \multicolumn{2}{|c|}{ Atom Atom } & \multirow{2}{*}{$\frac{\text { Length/Å }}{1.443(9)}$} \\
\hline $\mathrm{I} 4$ & $\mathrm{C} 2$ & & $\mathrm{C} 1$ & $\mathrm{C} 6$ & \\
\hline $\mathrm{I} 3$ & $\mathrm{C} 4$ & $2.104(6)$ & $\mathrm{C} 27$ & $\mathrm{C} 26$ & $1.439(9)$ \\
\hline $\mathrm{I} 2$ & $\mathrm{C} 28$ & $2.106(7)$ & $\mathrm{C} 26$ & $\mathrm{C} 25$ & $1.433(9)$ \\
\hline I1 & $\mathrm{C} 30$ & $2.102(7)$ & $\mathrm{C} 15$ & $\mathrm{C} 16$ & $1.417(10)$ \\
\hline Col & $\mathrm{O} 1$ & $2.037(4)$ & $\mathrm{C} 15$ & C14 & $1.436(10)$ \\
\hline Col & $\mathrm{O} 2$ & $2.029(5)$ & $\mathrm{C} 30$ & $\mathrm{C} 25$ & $1.413(10)$ \\
\hline Col & N4 & $2.190(6)$ & $\mathrm{C} 30$ & $\mathrm{C} 29$ & $1.355(10)$ \\
\hline Col & N2 & $2.138(5)$ & $\mathrm{C} 2$ & $\mathrm{C} 3$ & $1.397(9)$ \\
\hline Col & N1 & $2.135(5)$ & $\mathrm{C} 25$ & $\mathrm{C} 24$ & $1.390(10)$ \\
\hline Col & N3 & $2.166(6)$ & C6 & $\mathrm{C} 5$ & $1.414(8)$ \\
\hline $\mathrm{O} 1$ & $\mathrm{C} 1$ & $1.294(7)$ & $\mathrm{C} 23$ & $\mathrm{C} 24$ & $1.366(10)$ \\
\hline $\mathrm{O} 2$ & $\mathrm{C} 27$ & $1.303(7)$ & $\mathrm{C} 23$ & $\mathrm{C} 22$ & $1.402(10)$ \\
\hline $\mathrm{C} 28$ & $\mathrm{C} 27$ & $1.367(9)$ & $\mathrm{C} 5$ & C9 & $1.402(9)$ \\
\hline $\mathrm{C} 28$ & $\mathrm{C} 29$ & $1.388(9)$ & C11 & $\mathrm{C} 12$ & $1.362(11)$ \\
\hline N4 & $\mathrm{C} 14$ & $1.363(8)$ & C11 & $\mathrm{C} 10$ & $1.385(10)$ \\
\hline N4 & $\mathrm{C} 10$ & $1.311(8)$ & $\mathrm{C} 7$ & $\mathrm{C} 8$ & $1.390(9)$ \\
\hline $\mathrm{N} 2$ & $\mathrm{C} 26$ & $1.363(8)$ & C16 & $\mathrm{C} 17$ & $1.443(12)$ \\
\hline $\mathrm{N} 2$ & $\mathrm{C} 22$ & $1.318(9)$ & $\mathrm{C} 16$ & C19 & $1.410(12)$ \\
\hline N1 & C6 & $1.380(8)$ & C14 & C13 & $1.397(10)$ \\
\hline N1 & $\mathrm{C} 7$ & $1.311(8)$ & $\mathrm{C} 13$ & $\mathrm{C} 12$ & $1.388(11)$ \\
\hline $\mathrm{C} 4$ & $\mathrm{C} 5$ & $1.422(9)$ & $\mathrm{C} 13$ & $\mathrm{C} 18$ & $1.418(12)$ \\
\hline $\mathrm{C} 4$ & $\mathrm{C} 3$ & $1.347(10)$ & $\mathrm{C} 8$ & C9 & $1.354(10)$ \\
\hline N3 & $\mathrm{C} 15$ & $1.348(9)$ & $\mathrm{C} 18$ & $\mathrm{C} 17$ & $1.318(12)$ \\
\hline
\end{tabular}




\begin{tabular}{llllll}
\hline $\mathrm{N} 3$ & $\mathrm{C} 21$ & $1.324(9)$ & $\mathrm{C} 21$ & $\mathrm{C} 20$ & $1.370(11)$ \\
$\mathrm{C} 1$ & $\mathrm{C} 2$ & $1.378(9)$ & $\mathrm{C} 19$ & $\mathrm{C} 20$ & $1.352(12)$ \\
\hline
\end{tabular}

Table S42. Selected bond angles $\left(^{\circ}\right)$ for Co14.

\begin{tabular}{|c|c|c|c|c|c|c|c|}
\hline \multicolumn{3}{|c|}{ Atom Atom Atom } & \multirow{2}{*}{$\frac{\text { Angle }^{\circ}}{91.2(2)}$} & \multicolumn{3}{|c|}{ Atom Atom Atom } & \multirow{2}{*}{$\frac{\text { Angle }^{\circ}}{123.7(8)}$} \\
\hline $\mathrm{O} 1$ & Col & N4 & & N3 & $\mathrm{C} 15$ & $\mathrm{C} 16$ & \\
\hline $\mathrm{O} 1$ & Col & N2 & $98.07(19)$ & N3 & $\mathrm{C} 15$ & C14 & $118.3(6)$ \\
\hline $\mathrm{O} 1$ & Col & N1 & $79.34(19)$ & $\mathrm{C} 16$ & $\mathrm{C} 15$ & $\mathrm{C} 14$ & $118.0(8)$ \\
\hline $\mathrm{O} 1$ & Col & N3 & $166.2(2)$ & $\mathrm{C} 25$ & $\mathrm{C} 30$ & I1 & $120.6(6)$ \\
\hline $\mathrm{O} 2$ & Col 1 & $\mathrm{O} 1$ & $103.42(19)$ & $\mathrm{C} 29$ & $\mathrm{C} 30$ & I1 & $118.7(6)$ \\
\hline $\mathrm{O} 2$ & Col & N4 & $164.5(2)$ & $\mathrm{C} 29$ & $\mathrm{C} 30$ & $\mathrm{C} 25$ & $120.7(6)$ \\
\hline $\mathrm{O} 2$ & Col 1 & N2 & $80.1(2)$ & $\mathrm{C} 1$ & $\mathrm{C} 2$ & I4 & $117.4(5)$ \\
\hline $\mathrm{O} 2$ & Col & N1 & $97.28(19)$ & $\mathrm{C} 1$ & $\mathrm{C} 2$ & $\mathrm{C} 3$ & $123.2(6)$ \\
\hline $\mathrm{O} 2$ & Col 1 & N3 & $89.7(2)$ & $\mathrm{C} 3$ & $\mathrm{C} 2$ & $\mathrm{I} 4$ & $119.3(5)$ \\
\hline $\mathrm{N} 2$ & Col & N4 & $93.0(2)$ & $\mathrm{C} 30$ & $\mathrm{C} 25$ & $\mathrm{C} 26$ & $117.1(7)$ \\
\hline $\mathrm{N} 2$ & Co 1 & N3 & $88.0(2)$ & $\mathrm{C} 24$ & $\mathrm{C} 25$ & $\mathrm{C} 26$ & $116.7(7)$ \\
\hline N1 & Col & N4 & $90.4(2)$ & $\mathrm{C} 24$ & $\mathrm{C} 25$ & $\mathrm{C} 30$ & $126.2(7)$ \\
\hline N1 & Co1 & $\mathrm{N} 2$ & $175.8(2)$ & N1 & C6 & $\mathrm{C} 1$ & $114.8(5)$ \\
\hline N1 & Col & N3 & $95.2(2)$ & N1 & C6 & $\mathrm{C} 5$ & $121.6(6)$ \\
\hline N3 & Col & N4 & $76.1(2)$ & $\mathrm{C} 5$ & C6 & $\mathrm{C} 1$ & $123.7(6)$ \\
\hline $\mathrm{C} 1$ & $\mathrm{O} 1$ & Col & $114.9(4)$ & $\mathrm{C} 24$ & $\mathrm{C} 23$ & $\mathrm{C} 22$ & $118.8(7)$ \\
\hline $\mathrm{C} 27$ & $\mathrm{O} 2$ & Co1 & $114.1(4)$ & C6 & $\mathrm{C} 5$ & $\mathrm{C} 4$ & $116.5(6)$ \\
\hline $\mathrm{C} 27$ & $\mathrm{C} 28$ & $\mathrm{I} 2$ & $117.6(5)$ & C9 & $\mathrm{C} 5$ & $\mathrm{C} 4$ & $126.7(6)$ \\
\hline $\mathrm{C} 27$ & C28 & $\mathrm{C} 29$ & $124.0(7)$ & C9 & $\mathrm{C} 5$ & C6 & $116.9(6)$ \\
\hline $\mathrm{C} 29$ & C28 & $\mathrm{I} 2$ & $118.3(6)$ & $\mathrm{C} 12$ & C11 & $\mathrm{C} 10$ & $117.9(8)$ \\
\hline $\mathrm{C} 14$ & N4 & Co1 & $114.2(5)$ & N1 & $\mathrm{C} 7$ & $\mathrm{C} 8$ & $122.6(7)$ \\
\hline $\mathrm{C} 10$ & N4 & Co1 & $127.1(5)$ & $\mathrm{C} 15$ & $\mathrm{C} 16$ & $\mathrm{C} 17$ & $118.2(8)$ \\
\hline $\mathrm{C} 10$ & N4 & C14 & $118.7(6)$ & C19 & C16 & $\mathrm{C} 15$ & $115.2(9)$ \\
\hline $\mathrm{C} 26$ & N2 & Co1 & $109.9(4)$ & C19 & $\mathrm{C} 16$ & $\mathrm{C} 17$ & $126.5(9)$ \\
\hline $\mathrm{C} 22$ & N2 & Co1 & $130.5(5)$ & N4 & C14 & $\mathrm{C} 15$ & $116.6(7)$ \\
\hline $\mathrm{C} 22$ & N2 & $\mathrm{C} 26$ & $119.5(6)$ & N4 & $\mathrm{C} 14$ & C13 & $122.0(7)$ \\
\hline C6 & N1 & Co1 & $111.0(4)$ & $\mathrm{C} 13$ & $\mathrm{C} 14$ & $\mathrm{C} 15$ & $121.4(7)$ \\
\hline $\mathrm{C} 7$ & N1 & Col & $130.0(5)$ & C14 & C13 & C18 & $118.5(8)$ \\
\hline $\mathrm{C} 7$ & N1 & C6 & $118.8(6)$ & $\mathrm{C} 12$ & $\mathrm{C} 13$ & $\mathrm{C} 14$ & $116.7(8)$ \\
\hline $\mathrm{C} 5$ & $\mathrm{C} 4$ & I3 & $120.0(6)$ & $\mathrm{C} 12$ & C13 & C18 & $124.9(9)$ \\
\hline $\mathrm{C} 3$ & $\mathrm{C} 4$ & I3 & $119.0(5)$ & $\mathrm{C} 23$ & C24 & $\mathrm{C} 25$ & $121.1(7)$ \\
\hline $\mathrm{C} 3$ & $\mathrm{C} 4$ & $\mathrm{C} 5$ & $121.0(6)$ & $\mathrm{N} 2$ & $\mathrm{C} 22$ & $\mathrm{C} 23$ & $122.5(7)$ \\
\hline $\mathrm{C} 15$ & N3 & Co1 & $114.7(5)$ & C9 & $\mathrm{C} 8$ & $\mathrm{C} 7$ & $119.8(7)$ \\
\hline $\mathrm{C} 21$ & N3 & Col & $128.0(6)$ & $\mathrm{C} 8$ & C9 & $\mathrm{C} 5$ & $120.3(7)$ \\
\hline $\mathrm{C} 21$ & N3 & $\mathrm{C} 15$ & $117.3(7)$ & $\mathrm{C} 11$ & $\mathrm{C} 12$ & $\mathrm{C} 13$ & $121.4(8)$ \\
\hline $\mathrm{O} 1$ & $\mathrm{C} 1$ & $\mathrm{C} 2$ & $125.8(6)$ & N4 & $\mathrm{C} 10$ & $\mathrm{C} 11$ & $123.3(7)$ \\
\hline $\mathrm{O} 1$ & $\mathrm{C} 1$ & C6 & $119.7(6)$ & $\mathrm{C} 17$ & C18 & $\mathrm{C} 13$ & $121.4(9)$ \\
\hline $\mathrm{C} 2$ & $\mathrm{C} 1$ & C6 & $114.5(6)$ & $\mathrm{C} 18$ & $\mathrm{C} 17$ & $\mathrm{C} 16$ & $122.4(8)$ \\
\hline $\mathrm{O} 2$ & $\mathrm{C} 27$ & $\mathrm{C} 28$ & $125.6(6)$ & C30 & C29 & $\mathrm{C} 28$ & $120.8(7)$ \\
\hline
\end{tabular}




\begin{tabular}{cccccccc}
\hline $\mathrm{O} 2$ & $\mathrm{C} 27$ & $\mathrm{C} 26$ & $119.3(6)$ & $\mathrm{C} 4$ & $\mathrm{C} 3$ & $\mathrm{C} 2$ & $121.1(6)$ \\
$\mathrm{C} 28$ & $\mathrm{C} 27$ & $\mathrm{C} 26$ & $115.2(6)$ & $\mathrm{N} 3$ & $\mathrm{C} 21$ & $\mathrm{C} 20$ & $123.9(9)$ \\
$\mathrm{N} 2$ & $\mathrm{C} 26$ & $\mathrm{C} 27$ & $116.3(6)$ & $\mathrm{C} 20$ & $\mathrm{C} 19$ & $\mathrm{C} 16$ & $120.7(9)$ \\
$\mathrm{N} 2$ & $\mathrm{C} 26$ & $\mathrm{C} 25$ & $121.4(6)$ & $\mathrm{C} 19$ & $\mathrm{C} 20$ & $\mathrm{C} 21$ & $119.2(9)$ \\
$\mathrm{C} 25$ & $\mathrm{C} 26$ & $\mathrm{C} 27$ & $122.3(7)$ & & & & \\
\hline
\end{tabular}

Table S43. Crystal data and structure refinement details for Co15.

\begin{tabular}{ll}
\hline Empirical formula & $\mathrm{C}_{30} \mathrm{H}_{20} \mathrm{CoI}_{4} \mathrm{~N}_{4} \mathrm{O}_{4}$ \\
Formula weight & 1067.03 \\
Temperature/K & $293(2)$ \\
Crystal system & monoclinic \\
Space group & $\mathrm{P} 2{ }_{1} / \mathrm{n}$ \\
$\mathrm{a} / \AA$ & $13.3841(14)$ \\
$\mathrm{b} / \AA$ & $16.5578(17)$ \\
$\mathrm{c} / \AA$ & $16.1825(16)$ \\
$\alpha /{ }^{\circ}$ & 90 \\
$\beta /{ }^{\circ}$ & $113.480(3)$ \\
$\gamma /{ }^{\circ}$ & 90 \\
Volume/ $\AA^{3}$ & $3289.3(6)$ \\
$\mathrm{Z}$ & 4 \\
$\rho_{\text {calc }} /$ cm $^{3}$ & 2.155 \\
$\mu / \mathrm{mm}^{-1}$ & 4.317 \\
$\mathrm{~F}(000)$ & 1996.0 \\
Crystal size/mm & \\
Radiation & $0.3 \times 0.2 \times 0.11$ \\
$2 \Theta$ range for data collection/ & ${ }^{3} 5.936$ to 55.412 \\
Index ranges & $-17 \leq \mathrm{h} \leq 17,-21 \leq \mathrm{k} \leq 21,-19 \leq 1 \leq 21$ \\
Reflections collected & 50788 \\
Independent reflections & $7647\left[\mathrm{R}_{\text {int }}=0.1025, \mathrm{R}_{\text {sigma }}=0.0729\right]$ \\
Data/restraints/parameters & $7647 / 0 / 390$ \\
Goodness-of-fit on $\mathrm{F}^{2}$ & 1.032 \\
Final R indexes $[\mathrm{I}>=2 \sigma(\mathrm{I})]$ & $\mathrm{R}_{1}=0.0615, \mathrm{wR}_{2}=0.1386$ \\
Final R indexes [all data $]$ & $\mathrm{R}_{1}=0.1213, \mathrm{wR}_{2}=0.1653$ \\
Largest diff. peak/hole / e $\AA^{-3} 1.76 /-1.29$ \\
\hline
\end{tabular}

Table S44. Selected bond lengths ( $\AA$ ) for Co15.

\begin{tabular}{|c|c|c|c|}
\hline Atom Atom & Length/Å & Atom Atom & Length/Å \\
\hline C14 & $2.086(6)$ & $\mathrm{O} 3$ & $1.340(8)$ \\
\hline $\mathrm{C} 16$ & $2.092(7)$ & $\mathrm{O} 3$ & $1.414(10)$ \\
\hline $\mathrm{C} 25$ & $2.101(7)$ & $\mathrm{O} 4$ & $1.362(9)$ \\
\hline $\mathrm{C} 23$ & $2.093(7)$ & $\mathrm{C} 12$ & $1.425(11)$ \\
\hline
\end{tabular}




\begin{tabular}{cccccc}
\hline Co1 & O2 & $2.070(4)$ & C13 & C18 & $1.454(9)$ \\
Co1 & O1 & $2.063(4)$ & C7 & C8 & $1.373(10)$ \\
Co1 & $\mathrm{N} 4$ & $2.133(5)$ & C7 & C5 & $1.486(9)$ \\
Co1 & $\mathrm{N} 2$ & $2.137(5)$ & C4 & C3 & $1.354(10)$ \\
Co1 & $\mathrm{N} 1$ & $2.128(6)$ & $\mathrm{C} 20$ & $\mathrm{C} 21$ & $1.357(10)$ \\
$\mathrm{C} 11$ & $\mathrm{~N} 3$ & $2.143(6)$ & $\mathrm{C} 20$ & $\mathrm{C} 19$ & $1.402(10)$ \\
$\mathrm{O} 2$ & $\mathrm{C} 22$ & $1.306(8)$ & $\mathrm{C} 10$ & $\mathrm{C} 9$ & $1.359(10)$ \\
$\mathrm{O} 1$ & $\mathrm{C} 13$ & $1.299(7)$ & $\mathrm{C} 10$ & $\mathrm{C} 11$ & $1.370(11)$ \\
$\mathrm{N} 4$ & $\mathrm{C} 4$ & $1.345(8)$ & $\mathrm{C} 17$ & $\mathrm{C} 21$ & $1.418(9)$ \\
$\mathrm{N} 4$ & $\mathrm{C} 5$ & $1.328(8)$ & $\mathrm{C} 17$ & $\mathrm{C} 18$ & $1.412(9)$ \\
$\mathrm{C} 14$ & $\mathrm{C} 13$ & $1.389(9)$ & $\mathrm{C} 26$ & $\mathrm{C} 27$ & $1.418(10)$ \\
$\mathrm{C} 14$ & $\mathrm{C} 15$ & $1.408(10)$ & $\mathrm{C} 26$ & $\mathrm{C} 25$ & $1.416(10)$ \\
$\mathrm{C} 22$ & $\mathrm{C} 23$ & $1.395(10)$ & $\mathrm{C} 26$ & $\mathrm{C} 30$ & $1.406(11)$ \\
$\mathrm{C} 22$ & $\mathrm{C} 27$ & $1.437(10)$ & $\mathrm{C} 23$ & $\mathrm{C} 24$ & $1.388(10)$ \\
$\mathrm{N} 2$ & $\mathrm{C} 18$ & $1.348(8)$ & $\mathrm{C} 9$ & $\mathrm{C} 8$ & $1.392(10)$ \\
$\mathrm{N} 2$ & $\mathrm{C} 19$ & $1.325(8)$ & $\mathrm{C} 24$ & $\mathrm{C} 25$ & $1.363(11)$ \\
$\mathrm{C} 16$ & $\mathrm{C} 15$ & $1.355(10)$ & $\mathrm{C} 2$ & $\mathrm{C} 3$ & $1.387(10)$ \\
$\mathrm{C} 16$ & $\mathrm{C} 17$ & $1.414(10)$ & $\mathrm{C} 2$ & $\mathrm{C} 6$ & $1.399(10)$ \\
$\mathrm{N} 1$ & $\mathrm{C} 28$ & $1.320(9)$ & $\mathrm{C} 28$ & $\mathrm{C} 29$ & $1.413(12)$ \\
$\mathrm{N} 1$ & $\mathrm{C} 27$ & $1.359(8)$ & $\mathrm{C} 6$ & $\mathrm{C} 5$ & $1.385(9)$ \\
$\mathrm{N} 3$ & $\mathrm{C} 7$ & $1.366(8)$ & $\mathrm{C} 30$ & $\mathrm{C} 29$ & $1.355(12)$ \\
$\mathrm{N} 3$ & $\mathrm{C} 11$ & $1.328(9)$ & & & \\
\hline
\end{tabular}

Table S45. Selected bond angles $\left(^{\circ}\right)$ for Co15.

\begin{tabular}{|c|c|c|c|c|c|c|c|}
\hline \multicolumn{3}{|c|}{ Atom Atom Atom } & \multirow{2}{*}{$\frac{\text { Angle }^{\circ}}{97.0(2)}$} & \multicolumn{3}{|c|}{ Atom Atom Atom } & \multirow{2}{*}{$\frac{\text { Angle }^{\circ}}{122.1(6)}$} \\
\hline $\mathrm{O} 2$ & Col & N4 & & N3 & $\mathrm{C} 7$ & $\mathrm{C} 8$ & \\
\hline $\mathrm{O} 2$ & Col & N2 & $89.3(2)$ & N3 & $\mathrm{C} 7$ & $\mathrm{C} 5$ & $115.1(6)$ \\
\hline $\mathrm{O} 2$ & Col & N1 & $78.6(2)$ & $\mathrm{C} 8$ & $\mathrm{C} 7$ & $\mathrm{C} 5$ & $122.8(6)$ \\
\hline $\mathrm{O} 2$ & Co1 & N3 & $97.0(2)$ & N4 & $\mathrm{C} 4$ & $\mathrm{C} 3$ & $124.2(7)$ \\
\hline $\mathrm{O} 1$ & Col & $\mathrm{O} 2$ & $164.85(19)$ & $\mathrm{C} 21$ & $\mathrm{C} 20$ & C19 & $119.3(6)$ \\
\hline $\mathrm{O} 1$ & Col & N4 & $96.31(19)$ & C9 & $\mathrm{C} 10$ & $\mathrm{C} 11$ & $117.8(7)$ \\
\hline $\mathrm{O} 1$ & Col & N2 & 78.51(19) & $\mathrm{C} 16$ & $\mathrm{C} 17$ & $\mathrm{C} 21$ & $125.6(6)$ \\
\hline $\mathrm{O} 1$ & Col & N1 & $93.5(2)$ & $\mathrm{C} 18$ & $\mathrm{C} 17$ & $\mathrm{C} 16$ & $118.6(6)$ \\
\hline $\mathrm{O} 1$ & Col & N3 & $93.0(2)$ & $\mathrm{C} 18$ & $\mathrm{C} 17$ & $\mathrm{C} 21$ & $115.8(6)$ \\
\hline N4 & Col & N2 & $169.8(2)$ & $\mathrm{C} 20$ & $\mathrm{C} 21$ & $\mathrm{C} 17$ & $120.6(6)$ \\
\hline N4 & Col & N3 & $76.5(2)$ & $\mathrm{C} 25$ & $\mathrm{C} 26$ & $\mathrm{C} 27$ & $118.1(7)$ \\
\hline $\mathrm{N} 2$ & Col & N3 & $94.9(2)$ & $\mathrm{C} 30$ & $\mathrm{C} 26$ & $\mathrm{C} 27$ & $116.3(7)$ \\
\hline N1 & Col 1 & N4 & $93.3(2)$ & $\mathrm{C} 30$ & $\mathrm{C} 26$ & $\mathrm{C} 25$ & $125.6(7)$ \\
\hline N1 & Col & N2 & $95.7(2)$ & $\mathrm{C} 22$ & $\mathrm{C} 23$ & I1 & $118.8(5)$ \\
\hline N1 & Col & N3 & $168.4(2)$ & $\mathrm{C} 24$ & $\mathrm{C} 23$ & I1 & $117.9(5)$ \\
\hline $\mathrm{C} 22$ & $\mathrm{O} 2$ & Co1 & $114.4(4)$ & $\mathrm{C} 24$ & $\mathrm{C} 23$ & $\mathrm{C} 22$ & $123.2(7)$ \\
\hline C13 & $\mathrm{O} 1$ & Co1 & $114.0(4)$ & $\mathrm{O} 4$ & C9 & $\mathrm{C} 8$ & $114.2(7)$ \\
\hline $\mathrm{C} 4$ & N4 & Col & $125.9(5)$ & $\mathrm{C} 10$ & C9 & $\mathrm{O} 4$ & $126.1(7)$ \\
\hline $\mathrm{C} 5$ & $\mathrm{~N} 4$ & $\mathrm{Co} 1$ & $116.9(4)$ & $\mathrm{C} 10$ & $\mathrm{C} 9$ & $\mathrm{C} 8$ & $119.7(7)$ \\
\hline
\end{tabular}




\begin{tabular}{cccccccc}
\hline $\mathrm{C} 5$ & $\mathrm{~N} 4$ & $\mathrm{C} 4$ & $117.2(6)$ & $\mathrm{N} 2$ & $\mathrm{C} 18$ & $\mathrm{C} 13$ & $114.7(6)$ \\
$\mathrm{C} 13$ & $\mathrm{C} 14$ & $\mathrm{I} 4$ & $119.0(5)$ & $\mathrm{N} 2$ & $\mathrm{C} 18$ & $\mathrm{C} 17$ & $123.3(6)$ \\
$\mathrm{C} 13$ & $\mathrm{C} 14$ & $\mathrm{C} 15$ & $122.6(6)$ & $\mathrm{C} 17$ & $\mathrm{C} 18$ & $\mathrm{C} 13$ & $122.0(6)$ \\
$\mathrm{C} 15$ & $\mathrm{C} 14$ & $\mathrm{I} 4$ & $118.3(5)$ & $\mathrm{C} 25$ & $\mathrm{C} 24$ & $\mathrm{C} 23$ & $120.8(7)$ \\
$\mathrm{O} 2$ & $\mathrm{C} 22$ & $\mathrm{C} 23$ & $125.7(7)$ & $\mathrm{C} 7$ & $\mathrm{C} 8$ & $\mathrm{C} 9$ & $118.8(7)$ \\
$\mathrm{O} 2$ & $\mathrm{C} 22$ & $\mathrm{C} 27$ & $119.0(6)$ & $\mathrm{O} 3$ & $\mathrm{C} 2$ & $\mathrm{C} 3$ & $115.9(6)$ \\
$\mathrm{C} 23$ & $\mathrm{C} 22$ & $\mathrm{C} 27$ & $115.3(6)$ & $\mathrm{O} 3$ & $\mathrm{C} 2$ & $\mathrm{C} 6$ & $125.6(7)$ \\
$\mathrm{C} 18$ & $\mathrm{~N} 2$ & $\mathrm{C} 1$ & $112.4(4)$ & $\mathrm{C} 3$ & $\mathrm{C} 2$ & $\mathrm{C} 6$ & $118.4(6)$ \\
$\mathrm{C} 19$ & $\mathrm{~N} 2$ & $\mathrm{C} 1$ & $128.4(5)$ & $\mathrm{N} 2$ & $\mathrm{C} 19$ & $\mathrm{C} 20$ & $122.2(7)$ \\
$\mathrm{C} 19$ & $\mathrm{~N} 2$ & $\mathrm{C} 18$ & $118.9(6)$ & $\mathrm{N} 1$ & $\mathrm{C} 28$ & $\mathrm{C} 29$ & $120.8(8)$ \\
$\mathrm{C} 15$ & $\mathrm{C} 16$ & $\mathrm{I} 3$ & $119.1(6)$ & $\mathrm{N} 3$ & $\mathrm{C} 11$ & $\mathrm{C} 10$ & $125.1(7)$ \\
$\mathrm{C} 15$ & $\mathrm{C} 16$ & $\mathrm{C} 17$ & $120.2(6)$ & $\mathrm{N} 1$ & $\mathrm{C} 27$ & $\mathrm{C} 22$ & $115.7(6)$ \\
$\mathrm{C} 17$ & $\mathrm{C} 16$ & $\mathrm{I} 3$ & $120.6(5)$ & $\mathrm{N} 1$ & $\mathrm{C} 27$ & $\mathrm{C} 26$ & $122.2(6)$ \\
$\mathrm{C} 28$ & $\mathrm{~N} 1$ & $\mathrm{C} 11$ & $127.6(5)$ & $\mathrm{C} 26$ & $\mathrm{C} 27$ & $\mathrm{C} 22$ & $122.2(6)$ \\
$\mathrm{C} 28$ & $\mathrm{~N} 1$ & $\mathrm{C} 27$ & $120.1(6)$ & $\mathrm{C} 4$ & $\mathrm{C} 3$ & $\mathrm{C} 2$ & $118.7(7)$ \\
$\mathrm{C} 27$ & $\mathrm{~N} 1$ & $\mathrm{C} 1$ & $112.3(4)$ & $\mathrm{C} 26$ & $\mathrm{C} 25$ & $\mathrm{I} 2$ & $121.5(6)$ \\
$\mathrm{C} 7$ & $\mathrm{~N} 3$ & $\mathrm{C} 1$ & $115.6(4)$ & $\mathrm{C} 24$ & $\mathrm{C} 25$ & $\mathrm{I} 2$ & $118.2(6)$ \\
$\mathrm{C} 11$ & $\mathrm{~N} 3$ & $\mathrm{C} 1$ & $128.0(5)$ & $\mathrm{C} 24$ & $\mathrm{C} 25$ & $\mathrm{C} 26$ & $120.3(6)$ \\
$\mathrm{C} 11$ & $\mathrm{~N} 3$ & $\mathrm{C} 7$ & $116.5(6)$ & $\mathrm{C} 5$ & $\mathrm{C} 6$ & $\mathrm{C} 2$ & $118.2(6)$ \\
$\mathrm{C} 2$ & $\mathrm{O} 3$ & $\mathrm{C} 1$ & $119.1(6)$ & $\mathrm{C} 29$ & $\mathrm{C} 30$ & $\mathrm{C} 26$ & $120.6(7)$ \\
$\mathrm{C} 9$ & $\mathrm{O} 4$ & $\mathrm{C} 12$ & $118.7(8)$ & $\mathrm{N} 4$ & $\mathrm{C} 5$ & $\mathrm{C} 7$ & $115.9(6)$ \\
$\mathrm{O} 1$ & $\mathrm{C} 13$ & $\mathrm{C} 14$ & $125.2(6)$ & $\mathrm{N} 4$ & $\mathrm{C} 5$ & $\mathrm{C} 6$ & $123.3(6)$ \\
$\mathrm{O} 1$ & $\mathrm{C} 13$ & $\mathrm{C} 18$ & $119.5(6)$ & $\mathrm{C} 6$ & $\mathrm{C} 5$ & $\mathrm{C} 7$ & $120.7(6)$ \\
$\mathrm{C} 14$ & $\mathrm{C} 13$ & $\mathrm{C} 18$ & $115.3(6)$ & $\mathrm{C} 30$ & $\mathrm{C} 29$ & $\mathrm{C} 28$ & $120.1(8)$ \\
$\mathrm{C} 16$ & $\mathrm{C} 15$ & $\mathrm{C} 14$ & $121.3(7)$ & & & & \\
\hline
\end{tabular}

Table S46. Crystal data and structure refinement details for Co16.

\begin{tabular}{ll}
\hline Empirical formula & $\mathrm{C}_{30} \mathrm{H}_{20} \mathrm{CoI}_{5} \mathrm{~N}_{4} \mathrm{O}_{2}$ \\
Formula weight & 1161.93 \\
Temperature/K & $293(2)$ \\
Crystal system & orthorhombic \\
Space group & Cmce \\
$\mathrm{a} / \AA$ & $21.5869(8)$ \\
$\mathrm{b} / \AA$ & $16.2149(6)$ \\
$\mathrm{c} / \AA$ & $22.1995(8)$ \\
$\alpha /{ }^{\circ}$ & 90 \\
$\beta /{ }^{\circ}$ & 90 \\
$\gamma /{ }^{\circ}$ & 90 \\
$\mathrm{Volume} / \AA^{3}$ & $7770.5(5)$ \\
$\mathrm{Z}$ & 8 \\
$\rho_{\text {calc }} / \mathrm{cm}^{3}$ & 1.986 \\
$\mu / \mathrm{mm}^{-1}$ & 4.444 \\
\hline
\end{tabular}




\begin{tabular}{ll}
\hline $\mathrm{F}(000)$ & 4288.0 \\
Crystal size $/ \mathrm{mm}^{3}$ & $0.3 \times 0.2 \times 0.12$ \\
Radiation & $\mathrm{MoK} \alpha(\lambda=0.71073)$ \\
$2 \Theta$ range for data collection $/{ }^{\circ} 6.222$ to 55.136 \\
Index ranges & $-28 \leq \mathrm{h} \leq 28,-21 \leq \mathrm{k} \leq 21,-28 \leq 1 \leq 28$ \\
Reflections collected & 59546 \\
Independent reflections & $4616\left[\mathrm{R}_{\text {int }}=0.0574, \mathrm{R}_{\text {sigma }}=0.0310\right]$ \\
Data/restraints/parameters & $4616 / 0 / 193$ \\
Goodness-of-fit on $\mathrm{F}^{2}$ & 1.056 \\
Final R indexes $[\mathrm{I}>=2 \sigma(\mathrm{I})]$ & $\mathrm{R}_{1}=0.0514, \mathrm{wR}_{2}=0.1285$ \\
Final R indexes [all data] & $\mathrm{R}_{1}=0.0855, \mathrm{wR}_{2}=0.1432$ \\
Largest diff. peak/hole $/ \mathrm{e} \AA^{-3} 1.26 /-1.79$
\end{tabular}

Table S47. Selected bond lengths ( $\AA$ ) for Co16.

\begin{tabular}{|c|c|c|c|c|c|}
\hline Atom & Atom & Length/Å & Atom & Atom & Length/Å \\
\hline $\mathrm{I} 2$ & $\mathrm{C} 13$ & $2.092(7)$ & $\mathrm{C} 13$ & $\mathrm{C} 12$ & $1.399(10)$ \\
\hline $\mathrm{I} 3$ & C11 & $2.096(7)$ & $\mathrm{C} 11$ & $\mathrm{C} 12$ & $1.366(12)$ \\
\hline Co1 & $\mathrm{O} 1$ & $1.896(4)$ & $\mathrm{C} 11$ & $\mathrm{C} 10$ & $1.421(11)$ \\
\hline Co1 & $\mathrm{O} 1^{1}$ & $1.896(4)$ & C14 & $\mathrm{C} 15$ & $1.407(9)$ \\
\hline Col & N0AA & $1.920(5)$ & $\mathrm{C} 1$ & $\mathrm{C} 1^{1}$ & $1.452(13)$ \\
\hline Col & $\mathrm{N} \mathrm{AA}^{1}$ & $1.920(5)$ & $\mathrm{C} 1$ & $\mathrm{C} 2$ & $1.384(9)$ \\
\hline Col & $\mathrm{N} 2^{1}$ & $1.931(5)$ & $\mathrm{C} 15$ & $\mathrm{C} 10$ & $1.411(9)$ \\
\hline Col & $\mathrm{N} 2$ & $1.931(5)$ & $\mathrm{C} 7$ & $\mathrm{C} 8$ & $1.407(10)$ \\
\hline $\mathrm{O} 1$ & $\mathrm{C} 14$ & $1.318(7)$ & $\mathrm{C} 2$ & $\mathrm{C} 3$ & $1.374(10)$ \\
\hline N0AA & $\mathrm{C} 1$ & $1.369(8)$ & $\mathrm{C} 10$ & C9 & $1.406(11)$ \\
\hline N0AA & $\mathrm{C} 5$ & $1.339(8)$ & $\mathrm{C} 5$ & $\mathrm{C} 4$ & $1.364(10)$ \\
\hline $\mathrm{N} 2$ & C15 & $1.375(8)$ & $\mathrm{C} 4$ & $\mathrm{C} 3$ & $1.382(11)$ \\
\hline $\mathrm{N} 2$ & $\mathrm{C} 7$ & $1.318(9)$ & $\mathrm{C} 8$ & C9 & $1.332(12)$ \\
\hline C13 & C14 & $1.376(9)$ & C6 & $\mathrm{C} 3$ & $1.512(11)$ \\
\hline
\end{tabular}

$13 / 2-X,+Y, 1 / 2-Z$

Table S48. Selected bond angles $\left({ }^{\circ}\right)$ for Co16.

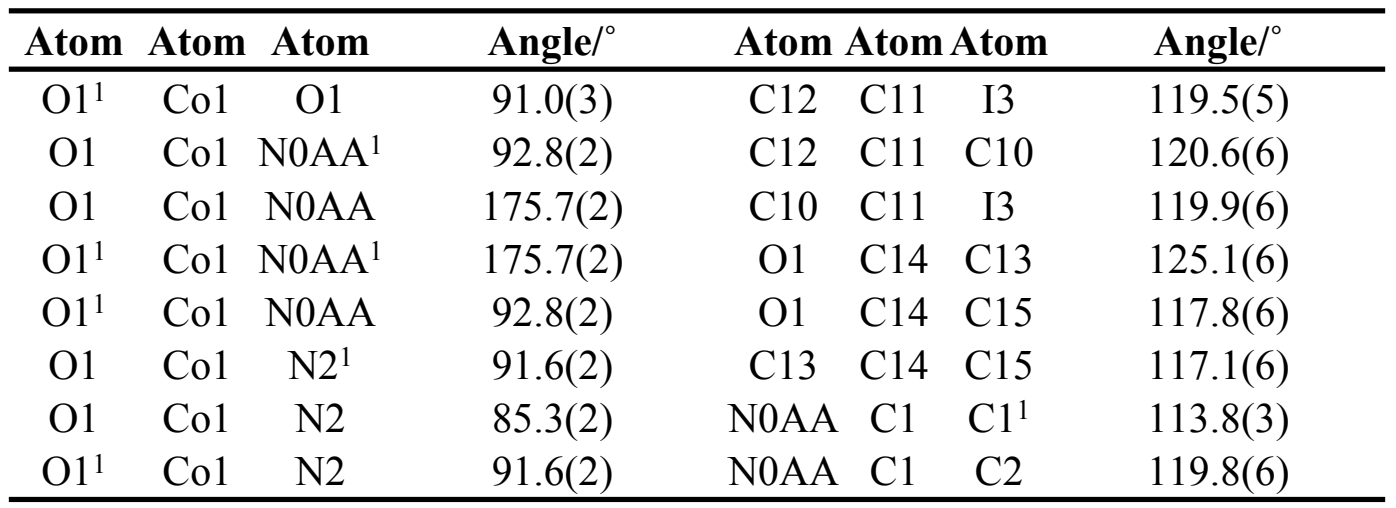




\begin{tabular}{|c|c|c|c|c|c|c|c|}
\hline $\mathrm{O} 1^{1}$ & Col & $\mathrm{N} 2^{1}$ & $85.3(2)$ & $\mathrm{C} 2$ & $\mathrm{C} 1$ & $\mathrm{C} 1^{1}$ & $126.3(4)$ \\
\hline $\mathrm{N}^{0 A A^{1}}{ }^{1}$ & Co1 & NOAA & $83.5(3)$ & C11 & $\mathrm{C} 12$ & C13 & $122.1(7)$ \\
\hline N0AA & Col & $\mathrm{N} 2^{1}$ & $90.8(2)$ & N2 & $\mathrm{C} 15$ & $\mathrm{C} 14$ & $114.4(5)$ \\
\hline N0AA & Col & N2 & $92.5(2)$ & N2 & $\mathrm{C} 15$ & $\mathrm{C} 10$ & $121.1(6)$ \\
\hline $\mathrm{N} \mathrm{AA}^{1}$ & Co1 & $\mathrm{N} 2$ & $90.8(2)$ & $\mathrm{C} 14$ & $\mathrm{C} 15$ & $\mathrm{C} 10$ & $124.5(6)$ \\
\hline $\mathrm{N} \mathrm{AA}^{1}$ & Col & $\mathrm{N} 2^{1}$ & $92.5(2)$ & N2 & $\mathrm{C} 7$ & $\mathrm{C} 8$ & $120.8(7)$ \\
\hline $\mathrm{N} 2^{1}$ & $\mathrm{Co} 1$ & N2 & $175.5(3)$ & $\mathrm{C} 3$ & $\mathrm{C} 2$ & $\mathrm{C} 1$ & $121.3(7)$ \\
\hline C14 & $\mathrm{O} 1$ & Col & $112.0(4)$ & C15 & $\mathrm{C} 10$ & C11 & $115.4(7)$ \\
\hline $\mathrm{C} 1 \mathrm{I}$ & N0AA & Col & $114.4(4)$ & C9 & $\mathrm{C} 10$ & C11 & $127.7(7)$ \\
\hline $\mathrm{C} 5 \mathrm{I}$ & NOAA & Col & $126.3(5)$ & C9 & $\mathrm{C} 10$ & C15 & $116.9(7)$ \\
\hline C5 I & NOAA & $\mathrm{C} 1$ & 119.1(6) & N0AA & $\mathrm{C} 5$ & $\mathrm{C} 4$ & $121.7(6)$ \\
\hline $\mathrm{C} 15$ & N2 & Col & $110.4(4)$ & $\mathrm{C} 5$ & $\mathrm{C} 4$ & $\mathrm{C} 3$ & $121.0(7)$ \\
\hline $\mathrm{C} 7$ & N2 & Col & $129.6(5)$ & C9 & $\mathrm{C} 8$ & $\mathrm{C} 7$ & $120.4(7)$ \\
\hline $\mathrm{C} 7$ & N2 & $\mathrm{C} 15$ & $120.0(5)$ & $\mathrm{C} 2$ & $\mathrm{C} 3$ & $\mathrm{C} 4$ & $117.0(7)$ \\
\hline $\mathrm{C} 14$ & $\mathrm{C} 13$ & $\mathrm{I} 2$ & $121.1(5)$ & $\mathrm{C} 2$ & $\mathrm{C} 3$ & C6 & $121.8(7)$ \\
\hline $\mathrm{C} 14$ & C13 & $\mathrm{C} 12$ & $120.3(7)$ & $\mathrm{C} 4$ & $\mathrm{C} 3$ & C6 & $121.2(7)$ \\
\hline $\mathrm{C} 12$ & $\mathrm{C} 13$ & $\mathrm{I} 2$ & $118.6(5)$ & $\mathrm{C} 8$ & C9 & $\mathrm{C} 10$ & $120.7(7)$ \\
\hline
\end{tabular}

$13 / 2-X,+Y, 1 / 2-Z$

Table S49. Crystal data and structure refinement details for Co17.

\begin{tabular}{ll}
\hline Empirical formula & $\mathrm{C}_{30} \mathrm{H}_{14} \mathrm{Cl}_{2} \mathrm{CoI}_{4} \mathrm{~N}_{4} \mathrm{O}_{2}$ \\
Formula weight & 1099.88 \\
Temperature/K & $293(2)$ \\
Crystal system & monoclinic \\
Space group & $\mathrm{P} 2{ }_{1} / \mathrm{c}$ \\
$\mathrm{a} / \AA$ & $12.1196(7)$ \\
$\mathrm{b} / \AA$ & $19.5096(12)$ \\
$\mathrm{c} / \AA$ & $15.2012(9)$ \\
$\alpha /{ }^{\circ}$ & 90 \\
$\beta /{ }^{\circ}$ & $109.306(2)$ \\
$\gamma /{ }^{\circ}$ & 90 \\
Volume/ ${ }^{3}$ & $3392.2(4)$ \\
$\mathrm{Z}$ & 4 \\
$\rho_{\text {calc }} \mathrm{g} /$ cm $^{3}$ & 2.154 \\
$\mu / \mathrm{mm}^{-1}$ & 4.337 \\
$\mathrm{~F}(000)$ & 2044.0 \\
Crystal size/mm & \\
Radiation & $0.26 \times 0.23 \times 0.19$ \\
$2 \Theta$ range for data collection/ & 5.994 to 55.21 \\
Index ranges & $-15 \leq \mathrm{h} \leq 15,-25 \leq \mathrm{k} \leq 25,-19 \leq 1 \leq 19$ \\
Reflections collected & 48666 \\
Independent reflections & $7837\left[\mathrm{R}_{\text {int }}=0.1173, \mathrm{R}_{\text {sigma }}=0.1110\right]$ \\
Data/restraints/parameters & $7837 / 0 / 388$ \\
\hline
\end{tabular}


Goodness-of-fit on $\mathrm{F}^{2} \quad 1.010$

Final $R$ indexes $[\mathrm{I}>=2 \sigma(\mathrm{I})] \quad \mathrm{R}_{1}=0.0777, \mathrm{wR}_{2}=0.1521$

Final $\mathrm{R}$ indexes [all data] $\quad \mathrm{R}_{1}=0.1868, \mathrm{wR}_{2}=0.1887$

Largest diff. peak/hole / e $\AA^{-3} 1.52 /-1.45$

Table S50. Selected bond lengths ( $\AA$ ) for Co17.

\begin{tabular}{|c|c|c|c|c|c|}
\hline \multicolumn{2}{|c|}{ Atom Atom } & \multirow{2}{*}{$\frac{\text { Length/Å }}{2.099(10)}$} & \multicolumn{2}{|c|}{ Atom Atom } & \multirow{2}{*}{$\frac{\text { Length/Å }}{1.420(16)}$} \\
\hline $\mathrm{I} 2$ & $\mathrm{C7}$ & & C17 & $\mathrm{C} 16$ & \\
\hline I1 & $\mathrm{C} 9$ & $2.100(10)$ & $\mathrm{C} 17$ & $\mathrm{C} 18$ & $1.346(17)$ \\
\hline I3 & $\mathrm{C} 16$ & $2.070(11)$ & N4 & $\mathrm{C} 24$ & $1.345(14)$ \\
\hline $\mathrm{I} 4$ & C18 & $2.101(12)$ & N4 & $\mathrm{C} 30$ & $1.358(17)$ \\
\hline Col & $\mathrm{O} 1$ & $2.040(6)$ & $\mathrm{C} 10$ & $\mathrm{C} 11$ & $1.410(18)$ \\
\hline Col & $\mathrm{O} 2$ & $2.037(6)$ & C9 & $\mathrm{C} 8$ & $1.382(14)$ \\
\hline Col & N1 & $2.126(9)$ & C9 & $\mathrm{C} 4$ & $1.393(13)$ \\
\hline Col & N3 & $2.155(9)$ & $\mathrm{C} 24$ & $\mathrm{C} 23$ & $1.413(16)$ \\
\hline Col & $\mathrm{N} 2$ & $2.121(9)$ & $\mathrm{C} 24$ & $\mathrm{C} 25$ & $1.412(17)$ \\
\hline Col & N4 & $2.138(10)$ & $\mathrm{C} 3$ & $\mathrm{C} 4$ & $1.421(14)$ \\
\hline $\mathrm{Cl1}$ & $\mathrm{C} 21$ & $1.757(12)$ & $\mathrm{C} 3$ & $\mathrm{C} 2$ & $1.366(14)$ \\
\hline $\mathrm{Cl} 2$ & $\mathrm{C} 28$ & $1.692(15)$ & $\mathrm{C} 8$ & $\mathrm{C} 7$ & $1.372(14)$ \\
\hline $\mathrm{O} 1$ & C6 & $1.278(11)$ & $\mathrm{C} 23$ & $\mathrm{C} 22$ & $1.409(16)$ \\
\hline $\mathrm{O} 2$ & $\mathrm{C} 15$ & $1.281(12)$ & $\mathrm{C} 1$ & $\mathrm{C} 2$ & $1.398(15)$ \\
\hline N1 & $\mathrm{C} 5$ & $1.358(12)$ & $\mathrm{C} 13$ & $\mathrm{C} 12$ & $1.401(17)$ \\
\hline N1 & $\mathrm{C} 1$ & $1.326(13)$ & C13 & C18 & $1.422(17)$ \\
\hline $\mathrm{C} 15$ & $\mathrm{C} 14$ & $1.439(15)$ & $\mathrm{C} 25$ & $\mathrm{C} 28$ & $1.40(2)$ \\
\hline $\mathrm{C} 15$ & $\mathrm{C} 16$ & $1.373(14)$ & $\mathrm{C} 25$ & $\mathrm{C} 26$ & $1.41(2)$ \\
\hline $\mathrm{C} 6$ & $\mathrm{C} 5$ & $1.447(13)$ & $\mathrm{C} 22$ & $\mathrm{C} 27$ & $1.41(2)$ \\
\hline C6 & $\mathrm{C} 7$ & $1.383(13)$ & $\mathrm{C} 22$ & $\mathrm{C} 21$ & $1.43(2)$ \\
\hline N3 & $\mathrm{C} 23$ & $1.350(13)$ & C19 & $\mathrm{C} 20$ & $1.412(17)$ \\
\hline N3 & C19 & $1.308(14)$ & $\mathrm{C} 28$ & C29 & $1.31(2)$ \\
\hline N2 & $\mathrm{C} 14$ & $1.364(13)$ & $\mathrm{C} 27$ & $\mathrm{C} 26$ & $1.36(2)$ \\
\hline $\mathrm{N} 2$ & $\mathrm{C} 10$ & $1.335(15)$ & $\mathrm{C} 12$ & $\mathrm{C} 11$ & $1.329(18)$ \\
\hline $\mathrm{C} 5$ & $\mathrm{C} 4$ & $1.428(14)$ & $\mathrm{C} 21$ & $\mathrm{C} 20$ & $1.242(18)$ \\
\hline $\mathrm{C} 14$ & $\mathrm{C} 13$ & $1.424(15)$ & $\mathrm{C} 30$ & C29 & $1.43(2)$ \\
\hline
\end{tabular}

Table S51. Selected bond angles $\left(^{\circ}\right)$ for Co17.

\begin{tabular}{cccccccc}
\hline \multicolumn{2}{|c}{ Atom Atom Atom } & \multicolumn{1}{c}{ Angle $^{\circ}$} & \multicolumn{4}{c}{ Atom Atom Atom } & Angle $^{\circ}$ \\
\hline $\mathrm{O} 1$ & $\mathrm{Co} 1$ & $\mathrm{~N} 1$ & $78.6(3)$ & $\mathrm{C} 15$ & $\mathrm{C} 16$ & $\mathrm{I} 3$ & $118.9(8)$ \\
$\mathrm{O} 1$ & $\mathrm{Co} 1$ & $\mathrm{~N} 3$ & $102.6(3)$ & $\mathrm{C} 15$ & $\mathrm{C} 16$ & $\mathrm{C} 17$ & $120.7(10)$ \\
$\mathrm{O} 1$ & $\mathrm{Co} 1$ & $\mathrm{~N} 2$ & $92.2(3)$ & $\mathrm{C} 17$ & $\mathrm{C} 16$ & $\mathrm{I} 3$ & $120.4(8)$ \\
$\mathrm{O} 1$ & $\mathrm{Co} 1$ & $\mathrm{~N} 4$ & $91.3(3)$ & $\mathrm{N} 4$ & $\mathrm{C} 24$ & $\mathrm{C} 23$ & $115.5(11)$ \\
\hline
\end{tabular}




\begin{tabular}{|c|c|c|c|c|c|c|c|}
\hline $\mathrm{O} 2$ & Co1 & $\mathrm{O} 1$ & $166.2(3)$ & N4 & $\mathrm{C} 24$ & $\mathrm{C} 25$ & $124.0(13)$ \\
\hline $\mathrm{O} 2$ & Co1 & N1 & $92.3(3)$ & $\mathrm{C} 25$ & $\mathrm{C} 24$ & $\mathrm{C} 23$ & $120.5(12)$ \\
\hline $\mathrm{O} 2$ & Col & N3 & $88.2(3)$ & $\mathrm{C} 2$ & $\mathrm{C} 3$ & $\mathrm{C} 4$ & $121.0(10)$ \\
\hline $\mathrm{O} 2$ & Co1 & $\mathrm{N} 2$ & $78.7(3)$ & $\mathrm{C} 7$ & $\mathrm{C} 8$ & C9 & $122.1(9)$ \\
\hline $\mathrm{O} 2$ & Co1 & N4 & $100.0(3)$ & C6 & $\mathrm{C} 7$ & $\mathrm{I} 2$ & $117.9(7)$ \\
\hline N1 & Co1 & N3 & $93.1(3)$ & $\mathrm{C} 8$ & $\mathrm{C} 7$ & $\mathrm{I} 2$ & $118.5(7)$ \\
\hline N1 & Co1 & N4 & $162.3(3)$ & $\mathrm{C} 8$ & $\mathrm{C} 7$ & C6 & $123.6(9)$ \\
\hline $\mathrm{N} 2$ & Co1 & N1 & 98.3(4) & N3 & $\mathrm{C} 23$ & $\mathrm{C} 24$ & $117.0(10)$ \\
\hline $\mathrm{N} 2$ & Col & N3 & $162.9(3)$ & N3 & $\mathrm{C} 23$ & $\mathrm{C} 22$ & $122.2(12)$ \\
\hline $\mathrm{N} 2$ & Co1 & N4 & $96.6(4)$ & $\mathrm{C} 22$ & $\mathrm{C} 23$ & $\mathrm{C} 24$ & $120.7(11)$ \\
\hline N4 & Co1 & N3 & $74.8(4)$ & N1 & $\mathrm{C} 1$ & $\mathrm{C} 2$ & $122.8(10)$ \\
\hline C6 & $\mathrm{O} 1$ & Col & $115.2(6)$ & $\mathrm{C} 12$ & $\mathrm{C} 13$ & $\mathrm{C} 14$ & $116.5(11)$ \\
\hline $\mathrm{C} 15$ & $\mathrm{O} 2$ & Co1 & $114.3(6)$ & $\mathrm{C} 12$ & $\mathrm{C} 13$ & $\mathrm{C} 18$ & $127.6(12)$ \\
\hline $\mathrm{C} 5$ & N1 & Co1 & $112.0(6)$ & C18 & $\mathrm{C} 13$ & $\mathrm{C} 14$ & $115.9(11)$ \\
\hline $\mathrm{C} 1$ & N1 & Co1 & $128.4(7)$ & C9 & $\mathrm{C} 4$ & $\mathrm{C} 5$ & $117.8(9)$ \\
\hline $\mathrm{C} 1$ & N1 & $\mathrm{C} 5$ & $119.4(9)$ & C9 & $\mathrm{C} 4$ & $\mathrm{C} 3$ & $126.4(10)$ \\
\hline $\mathrm{O} 2$ & $\mathrm{C} 15$ & $\mathrm{C} 14$ & $120.7(9)$ & $\mathrm{C} 3$ & $\mathrm{C} 4$ & $\mathrm{C} 5$ & $115.9(9)$ \\
\hline $\mathrm{O} 2$ & $\mathrm{C} 15$ & $\mathrm{C} 16$ & $123.4(10)$ & $\mathrm{C} 3$ & $\mathrm{C} 2$ & $\mathrm{C} 1$ & $118.8(10)$ \\
\hline $\mathrm{C} 16$ & $\mathrm{C} 15$ & $\mathrm{C} 14$ & $115.8(10)$ & $\mathrm{C} 28$ & $\mathrm{C} 25$ & $\mathrm{C} 24$ & $117.9(13)$ \\
\hline $\mathrm{O} 1$ & C6 & $\mathrm{C} 5$ & $119.5(8)$ & C28 & $\mathrm{C} 25$ & $\mathrm{C} 26$ & $123.8(14)$ \\
\hline $\mathrm{O} 1$ & C6 & $\mathrm{C} 7$ & $126.8(9)$ & C26 & $\mathrm{C} 25$ & $\mathrm{C} 24$ & $118.3(15)$ \\
\hline $\mathrm{C} 7$ & C6 & $\mathrm{C} 5$ & $113.7(9)$ & $\mathrm{C} 23$ & $\mathrm{C} 22$ & $\mathrm{C} 27$ & $116.6(14)$ \\
\hline $\mathrm{C} 23$ & N3 & Co1 & $115.4(8)$ & $\mathrm{C} 23$ & $\mathrm{C} 22$ & $\mathrm{C} 21$ & $115.1(12)$ \\
\hline C19 & N3 & Co1 & $127.1(8)$ & $\mathrm{C} 27$ & $\mathrm{C} 22$ & $\mathrm{C} 21$ & $128.3(14)$ \\
\hline C19 & N3 & $\mathrm{C} 23$ & $117.1(10)$ & N3 & $\mathrm{C} 19$ & $\mathrm{C} 20$ & $123.9(13)$ \\
\hline $\mathrm{C} 14$ & $\mathrm{~N} 2$ & Col & $112.5(7)$ & $\mathrm{C} 25$ & $\mathrm{C} 28$ & $\mathrm{Cl} 2$ & $121.0(13)$ \\
\hline $\mathrm{C} 10$ & $\mathrm{~N} 2$ & Co1 & $129.3(8)$ & C29 & $\mathrm{C} 28$ & $\mathrm{Cl} 2$ & $119.9(14)$ \\
\hline $\mathrm{C} 10$ & $\mathrm{~N} 2$ & $\mathrm{C} 14$ & $118.0(10)$ & C29 & $\mathrm{C} 28$ & $\mathrm{C} 25$ & $119.1(14)$ \\
\hline N1 & $\mathrm{C} 5$ & C6 & $114.4(9)$ & C26 & $\mathrm{C} 27$ & $\mathrm{C} 22$ & $123.6(14)$ \\
\hline N1 & $\mathrm{C} 5$ & $\mathrm{C} 4$ & $122.2(9)$ & $\mathrm{C} 11$ & $\mathrm{C} 12$ & $\mathrm{C} 13$ & $120.6(13)$ \\
\hline $\mathrm{C} 4$ & $\mathrm{C} 5$ & C6 & $123.4(9)$ & $\mathrm{C} 17$ & $\mathrm{C} 18$ & I4 & 119.9(9) \\
\hline N2 & $\mathrm{C} 14$ & $\mathrm{C} 15$ & $113.2(9)$ & $\mathrm{C} 17$ & $\mathrm{C} 18$ & $\mathrm{C} 13$ & $120.3(11)$ \\
\hline $\mathrm{N} 2$ & $\mathrm{C} 14$ & $\mathrm{C} 13$ & $122.6(10)$ & $\mathrm{C} 13$ & $\mathrm{C} 18$ & $\mathrm{I} 4$ & $119.8(10)$ \\
\hline $\mathrm{C} 13$ & $\mathrm{C} 14$ & $\mathrm{C} 15$ & $124.2(10)$ & $\mathrm{C} 22$ & $\mathrm{C} 21$ & $\mathrm{Cl1}$ & $116.1(12)$ \\
\hline C18 & $\mathrm{C} 17$ & $\mathrm{C} 16$ & $123.0(10)$ & $\mathrm{C} 20$ & $\mathrm{C} 21$ & $\mathrm{C} 11$ & $121.1(13)$ \\
\hline $\mathrm{C} 24$ & N4 & Co1 & $117.1(8)$ & $\mathrm{C} 20$ & $\mathrm{C} 21$ & $\mathrm{C} 22$ & $122.7(12)$ \\
\hline $\mathrm{C} 24$ & N4 & C30 & $116.2(11)$ & $\mathrm{C} 21$ & $\mathrm{C} 20$ & C19 & $118.9(13)$ \\
\hline $\mathrm{C} 30$ & N4 & Co1 & $126.7(9)$ & N4 & $\mathrm{C} 30$ & $\mathrm{C} 29$ & $121.6(14)$ \\
\hline $\mathrm{N} 2$ & $\mathrm{C} 10$ & $\mathrm{C} 11$ & $121.6(12)$ & $\mathrm{C} 27$ & $\mathrm{C} 26$ & $\mathrm{C} 25$ & $120.1(15)$ \\
\hline $\mathrm{C} 8$ & C9 & I1 & $118.6(7)$ & $\mathrm{C} 12$ & $\mathrm{C} 11$ & $\mathrm{C} 10$ & $120.5(13)$ \\
\hline $\mathrm{C} 8$ & C9 & $\mathrm{C} 4$ & $119.2(10)$ & C28 & $\mathrm{C} 29$ & C30 & $121.3(15)$ \\
\hline $\mathrm{C} 4$ & C9 & I1 & $122.1(8)$ & & & & \\
\hline
\end{tabular}


Table S52. Crystal data and structure refinement details for Co18.

\begin{tabular}{|c|c|}
\hline Empirical formula & $\mathrm{C}_{31} \mathrm{H}_{18} \mathrm{Br}_{4} \mathrm{Cl}_{2} \mathrm{CoN}_{4} \mathrm{O}_{3}$ \\
\hline Formula weight & 943.96 \\
\hline Temperature/K & 293(2) \\
\hline Crystal system & orthorhombic \\
\hline Space group & Pbca \\
\hline $\mathrm{a} / \AA$ & $13.1807(7)$ \\
\hline $\mathrm{b} / \AA$ & $16.4645(9)$ \\
\hline $\mathrm{c} / \AA$ & $30.0081(17)$ \\
\hline$\alpha /^{\circ}$ & 90 \\
\hline$\beta /{ }^{\circ}$ & 90 \\
\hline$\gamma /{ }^{\circ}$ & 90 \\
\hline Volume $/ \AA^{3}$ & $6512.2(6)$ \\
\hline $\mathrm{Z}$ & 8 \\
\hline$\rho_{\text {calc }} \mathrm{g} / \mathrm{cm}^{3}$ & 1.926 \\
\hline$\mu / \mathrm{mm}^{-1}$ & 5.640 \\
\hline $\mathrm{F}(000)$ & 3656.0 \\
\hline Crystal size $/ \mathrm{mm}^{3}$ & $0.35 \times 0.33 \times 0.29$ \\
\hline Radiation & $\operatorname{MoK} \alpha(\lambda=0.71073)$ \\
\hline \multicolumn{2}{|c|}{$2 \Theta$ range for data collection $/{ }^{\circ} 6.182$ to 55.072} \\
\hline Index ranges & $-16 \leq \mathrm{h} \leq 17,-21 \leq \mathrm{k} \leq 21,-38 \leq 1 \leq 39$ \\
\hline Reflections collected & 98683 \\
\hline Independent reflections & $7497\left[\mathrm{R}_{\text {int }}=0.0933, \mathrm{R}_{\text {sigma }}=0.0512\right]$ \\
\hline Data/restraints/parameters & $7497 / 0 / 408$ \\
\hline Goodness-of-fit on $\mathrm{F}^{2}$ & 1.070 \\
\hline Final $\mathrm{R}$ indexes $[\mathrm{I}>=2 \sigma(\mathrm{I})]$ & $\mathrm{R}_{1}=0.0541, \mathrm{wR}_{2}=0.0990$ \\
\hline Final $\mathrm{R}$ indexes [all data] & $\mathrm{R}_{1}=0.1184, \mathrm{wR}_{2}=0.1255$ \\
\hline Largest diff. peak/hole / e $\AA$ & $1.22 /-1.20$ \\
\hline
\end{tabular}

Table S53. Selected bond lengths ( $\AA$ ) for Co18.

\begin{tabular}{|c|c|c|c|}
\hline Atom Atom & Length/Å & Atom Atom & Length/Å \\
\hline $\begin{array}{ll}\mathrm{Co} 1 \mathrm{O} 2 \\
\end{array}$ & $2.065(3)$ & $\mathrm{C} 1$ & $1.389(8)$ \\
\hline Co1 O1 & $2.036(3)$ & $\mathrm{C} 3$ & $1.357(9)$ \\
\hline Co1 N4 & $2.141(4)$ & C14 C15 & $1.438(7)$ \\
\hline Co1 N3 & $2.134(4)$ & C14 C13 & $1.417(6)$ \\
\hline Col & $2.124(4)$ & $\mathrm{C} 4$ & $1.416(7)$ \\
\hline Col & $2.122(4)$ & C15 C16 & $1.384(7)$ \\
\hline $\mathrm{Br} 4 \quad \mathrm{C} 18$ & $1.898(5)$ & $\mathrm{C} 23 \mathrm{C} 24$ & $1.438(7)$ \\
\hline $\mathrm{Br} 3 \quad \mathrm{C} 16$ & $1.891(5)$ & $\mathrm{C} 23 \mathrm{C} 22$ & $1.395(7)$ \\
\hline $\mathrm{Br} 1 \quad \mathrm{C} 7$ & $1.879(6)$ & $\mathrm{C} 24 \mathrm{C} 25$ & $1.403(7)$ \\
\hline $\mathrm{Br} 2 \quad \mathrm{C} 9$ & $1.898(5)$ & C13 C12 & $1.409(8)$ \\
\hline
\end{tabular}




\begin{tabular}{cccccc}
\hline $\mathrm{C} 12$ & $\mathrm{C} 28$ & $1.732(5)$ & $\mathrm{C} 25$ & $\mathrm{C} 28$ & $1.398(8)$ \\
$\mathrm{C} 11$ & $\mathrm{C} 21$ & $1.728(6)$ & $\mathrm{C} 25$ & $\mathrm{C} 26$ & $1.429(8)$ \\
$\mathrm{O} 2$ & $\mathrm{C} 15$ & $1.301(6)$ & $\mathrm{C} 22$ & $\mathrm{C} 21$ & $1.415(8)$ \\
$\mathrm{O} 1$ & $\mathrm{C} 6$ & $1.295(6)$ & $\mathrm{C} 22$ & $\mathrm{C} 27$ & $1.424(8)$ \\
$\mathrm{N} 4$ & $\mathrm{C} 24$ & $1.355(6)$ & $\mathrm{C} 30$ & $\mathrm{C} 29$ & $1.389(8)$ \\
$\mathrm{N} 4$ & $\mathrm{C} 30$ & $1.323(6)$ & $\mathrm{C} 16$ & $\mathrm{C} 17$ & $1.403(7)$ \\
$\mathrm{N} 3$ & $\mathrm{C} 23$ & $1.356(6)$ & $\mathrm{C} 28$ & $\mathrm{C} 29$ & $1.362(9)$ \\
$\mathrm{N} 3$ & $\mathrm{C} 19$ & $1.323(6)$ & $\mathrm{C} 4$ & $\mathrm{C} 9$ & $1.406(8)$ \\
$\mathrm{N} 2$ & $\mathrm{C} 14$ & $1.359(6)$ & $\mathrm{C} 4$ & $\mathrm{C} 3$ & $1.407(8)$ \\
$\mathrm{N} 2$ & $\mathrm{C} 10$ & $1.322(6)$ & $\mathrm{C} 12$ & $\mathrm{C} 11$ & $1.359(8)$ \\
$\mathrm{N} 1$ & $\mathrm{C} 5$ & $1.361(6)$ & $\mathrm{C} 9$ & $\mathrm{C} 8$ & $1.353(9)$ \\
$\mathrm{N} 1$ & $\mathrm{C} 1$ & $1.325(6)$ & $\mathrm{C} 10$ & $\mathrm{C} 11$ & $1.393(7)$ \\
$\mathrm{O} 3$ & $\mathrm{C} 31$ & $1.401(7)$ & $\mathrm{C} 26$ & $\mathrm{C} 27$ & $1.341(9)$ \\
$\mathrm{C} 6$ & $\mathrm{C} 5$ & $1.432(7)$ & $\mathrm{C} 19$ & $\mathrm{C} 20$ & $1.378(8)$ \\
$\mathrm{C} 6$ & $\mathrm{C} 7$ & $1.392(7)$ & $\mathrm{C} 7$ & $\mathrm{C} 8$ & $1.398(7)$ \\
$\mathrm{C} 18$ & $\mathrm{C} 13$ & $1.412(8)$ & $\mathrm{C} 21$ & $\mathrm{C} 20$ & $1.344(9)$ \\
$\mathrm{C} 18$ & $\mathrm{C} 17$ & $1.352(8)$ & & & \\
\hline
\end{tabular}

Table S54. Selected bond angles $\left(^{\circ}\right)$ for Co18.

\begin{tabular}{|c|c|c|c|c|c|c|c|}
\hline \multicolumn{3}{|c|}{ Atom Atom Atom } & \multirow{2}{*}{$\begin{array}{c}\text { Angle }^{\circ} \\
93.72(14)\end{array}$} & \multicolumn{3}{|c|}{ Atom Atom Atom } & \multirow{2}{*}{$\frac{\text { Angle }^{\circ}}{123.2(5)}$} \\
\hline $\mathrm{O} 2$ & Col & N4 & & N3 & $\mathrm{C} 23$ & $\mathrm{C} 22$ & \\
\hline $\mathrm{O} 2$ & Col & N3 & $96.07(15)$ & $\mathrm{C} 22$ & $\mathrm{C} 23$ & $\mathrm{C} 24$ & $120.2(5)$ \\
\hline $\mathrm{O} 2$ & Col & $\mathrm{N} 2$ & $78.90(15)$ & N4 & $\mathrm{C} 24$ & $\mathrm{C} 23$ & $117.5(4)$ \\
\hline $\mathrm{O} 2$ & Col & N1 & $90.40(15)$ & N4 & $\mathrm{C} 24$ & $\mathrm{C} 25$ & $123.4(5)$ \\
\hline $\mathrm{O} 1$ & Co1 & $\mathrm{O} 2$ & $165.93(14)$ & $\mathrm{C} 25$ & $\mathrm{C} 24$ & $\mathrm{C} 23$ & $119.1(5)$ \\
\hline $\mathrm{O} 1$ & Col & N4 & $96.89(15)$ & $\mathrm{C} 18$ & $\mathrm{C} 13$ & $\mathrm{C} 14$ & $117.4(5)$ \\
\hline $\mathrm{O} 1$ & Col & N3 & $95.25(15)$ & $\mathrm{C} 12$ & $\mathrm{C} 13$ & $\mathrm{C} 18$ & $125.9(5)$ \\
\hline $\mathrm{O} 1$ & Col & $\mathrm{N} 2$ & $91.90(15)$ & $\mathrm{C} 12$ & $\mathrm{C} 13$ & $\mathrm{C} 14$ & $116.6(5)$ \\
\hline $\mathrm{O} 1$ & Col & N1 & $79.51(14)$ & $\mathrm{C} 24$ & $\mathrm{C} 25$ & $\mathrm{C} 26$ & $118.7(5)$ \\
\hline N3 & Col & N4 & $77.29(16)$ & $\mathrm{C} 28$ & $\mathrm{C} 25$ & $\mathrm{C} 24$ & $115.8(5)$ \\
\hline N2 & Col & N4 & $168.52(16)$ & $\mathrm{C} 28$ & $\mathrm{C} 25$ & $\mathrm{C} 26$ & $125.5(5)$ \\
\hline N2 & Col & N3 & $94.62(15)$ & N1 & $\mathrm{C} 1$ & $\mathrm{C} 2$ & $122.3(5)$ \\
\hline N1 & Col & N4 & $95.07(15)$ & $\mathrm{C} 23$ & $\mathrm{C} 22$ & $\mathrm{C} 21$ & $116.1(5)$ \\
\hline N1 & Col & N3 & $170.25(16)$ & $\mathrm{C} 23$ & $\mathrm{C} 22$ & $\mathrm{C} 27$ & $119.3(5)$ \\
\hline N1 & Col & $\mathrm{N} 2$ & $93.77(15)$ & $\mathrm{C} 21$ & $\mathrm{C} 22$ & $\mathrm{C} 27$ & $124.6(5)$ \\
\hline $\mathrm{C} 15$ & $\mathrm{O} 2$ & Col & $113.5(3)$ & N4 & $\mathrm{C} 30$ & $\mathrm{C} 29$ & $123.7(6)$ \\
\hline C6 & O1 & Col & $113.8(3)$ & $\mathrm{C} 15$ & $\mathrm{C} 16$ & $\mathrm{Br} 3$ & $118.2(4)$ \\
\hline $\mathrm{C} 24$ & N4 & Co1 & $114.0(3)$ & $\mathrm{C} 15$ & $\mathrm{C} 16$ & $\mathrm{C} 17$ & $123.2(5)$ \\
\hline $\mathrm{C} 30$ & N4 & Col & $128.3(4)$ & $\mathrm{C} 17$ & $\mathrm{C} 16$ & $\mathrm{Br} 3$ & $118.6(4)$ \\
\hline $\mathrm{C} 30$ & N4 & $\mathrm{C} 24$ & $117.7(4)$ & $\mathrm{C} 25$ & $\mathrm{C} 28$ & $\mathrm{Cl} 2$ & $119.9(5)$ \\
\hline $\mathrm{C} 23$ & N3 & Co1 & $114.7(3)$ & $\mathrm{C} 29$ & $\mathrm{C} 28$ & $\mathrm{Cl} 2$ & $118.5(5)$ \\
\hline
\end{tabular}




\begin{tabular}{cccccccc}
\hline $\mathrm{C} 19$ & $\mathrm{~N} 3$ & $\mathrm{C} 1$ & $127.8(4)$ & $\mathrm{C} 29$ & $\mathrm{C} 28$ & $\mathrm{C} 25$ & $121.6(5)$ \\
$\mathrm{C} 19$ & $\mathrm{~N} 3$ & $\mathrm{C} 23$ & $117.5(4)$ & $\mathrm{C} 9$ & $\mathrm{C} 4$ & $\mathrm{C} 5$ & $117.3(5)$ \\
$\mathrm{C} 14$ & $\mathrm{~N} 2$ & $\mathrm{C} 1$ & $112.1(3)$ & $\mathrm{C} 9$ & $\mathrm{C} 4$ & $\mathrm{C} 3$ & $126.2(5)$ \\
$\mathrm{C} 10$ & $\mathrm{~N} 2$ & $\mathrm{C} 1$ & $128.8(4)$ & $\mathrm{C} 3$ & $\mathrm{C} 4$ & $\mathrm{C} 5$ & $116.5(5)$ \\
$\mathrm{C} 10$ & $\mathrm{~N} 2$ & $\mathrm{C} 14$ & $119.0(4)$ & $\mathrm{C} 11$ & $\mathrm{C} 12$ & $\mathrm{C} 13$ & $120.1(5)$ \\
$\mathrm{C} 5$ & $\mathrm{~N} 1$ & $\mathrm{C} 1$ & $111.6(3)$ & $\mathrm{C} 4$ & $\mathrm{C} 9$ & $\mathrm{Br} 2$ & $120.4(5)$ \\
$\mathrm{C} 1$ & $\mathrm{~N} 1$ & $\mathrm{C} 1$ & $129.4(4)$ & $\mathrm{C} 8$ & $\mathrm{C} 9$ & $\mathrm{Br} 2$ & $118.7(5)$ \\
$\mathrm{C} 1$ & $\mathrm{~N} 1$ & $\mathrm{C} 5$ & $119.0(4)$ & $\mathrm{C} 8$ & $\mathrm{C} 9$ & $\mathrm{C} 4$ & $120.9(5)$ \\
$\mathrm{O} 1$ & $\mathrm{C} 6$ & $\mathrm{C} 5$ & $120.5(4)$ & $\mathrm{N} 2$ & $\mathrm{C} 10$ & $\mathrm{C} 11$ & $122.3(5)$ \\
$\mathrm{O} 1$ & $\mathrm{C} 6$ & $\mathrm{C} 7$ & $124.6(5)$ & $\mathrm{C} 18$ & $\mathrm{C} 17$ & $\mathrm{C} 16$ & $120.5(5)$ \\
$\mathrm{C} 7$ & $\mathrm{C} 6$ & $\mathrm{C} 5$ & $114.9(4)$ & $\mathrm{C} 27$ & $\mathrm{C} 26$ & $\mathrm{C} 25$ & $122.1(5)$ \\
$\mathrm{C} 13$ & $\mathrm{C} 18$ & $\mathrm{~B} 4$ & $119.7(4)$ & $\mathrm{N} 3$ & $\mathrm{C} 19$ & $\mathrm{C} 20$ & $123.7(5)$ \\
$\mathrm{C} 17$ & $\mathrm{C} 18$ & $\mathrm{~B} 4$ & $119.4(4)$ & $\mathrm{C} 6$ & $\mathrm{C} 7$ & $\mathrm{Br} 1$ & $118.7(4)$ \\
$\mathrm{C} 17$ & $\mathrm{C} 18$ & $\mathrm{C} 13$ & $121.0(5)$ & $\mathrm{C} 6$ & $\mathrm{C} 7$ & $\mathrm{C} 8$ & $122.6(5)$ \\
$\mathrm{C} 3$ & $\mathrm{C} 2$ & $\mathrm{C} 1$ & $119.6(6)$ & $\mathrm{C} 8$ & $\mathrm{C} 7$ & $\mathrm{Br} 1$ & $118.6(4)$ \\
$\mathrm{N} 2$ & $\mathrm{C} 14$ & $\mathrm{C} 15$ & $114.9(4)$ & $\mathrm{C} 22$ & $\mathrm{C} 21$ & $\mathrm{C} 11$ & $119.6(5)$ \\
$\mathrm{N} 2$ & $\mathrm{C} 14$ & $\mathrm{C} 13$ & $122.2(5)$ & $\mathrm{C} 20$ & $\mathrm{C} 21$ & $\mathrm{C} 11$ & $119.7(5)$ \\
$\mathrm{C} 13$ & $\mathrm{C} 14$ & $\mathrm{C} 15$ & $122.9(5)$ & $\mathrm{C} 20$ & $\mathrm{C} 21$ & $\mathrm{C} 22$ & $120.6(5)$ \\
$\mathrm{N} 1$ & $\mathrm{C} 5$ & $\mathrm{C} 6$ & $114.6(4)$ & $\mathrm{C} 28$ & $\mathrm{C} 29$ & $\mathrm{C} 30$ & $117.8(6)$ \\
$\mathrm{N} 1$ & $\mathrm{C} 5$ & $\mathrm{C} 4$ & $122.1(5)$ & $\mathrm{C} 9$ & $\mathrm{C} 8$ & $\mathrm{C} 7$ & $121.0(5)$ \\
$\mathrm{C} 4$ & $\mathrm{C} 5$ & $\mathrm{C} 6$ & $123.2(5)$ & $\mathrm{C} 12$ & $\mathrm{C} 11$ & $\mathrm{C} 10$ & $119.7(5)$ \\
$\mathrm{O} 2$ & $\mathrm{C} 15$ & $\mathrm{C} 14$ & $119.9(4)$ & $\mathrm{C} 2$ & $\mathrm{C} 3$ & $\mathrm{C} 4$ & $120.5(5)$ \\
$\mathrm{O} 2$ & $\mathrm{C} 15$ & $\mathrm{C} 16$ & $125.2(5)$ & $\mathrm{C} 21$ & $\mathrm{C} 20$ & $\mathrm{C} 19$ & $118.9(6)$ \\
$\mathrm{C} 16$ & $\mathrm{C} 15$ & $\mathrm{C} 14$ & $114.9(4)$ & $\mathrm{C} 26$ & $\mathrm{C} 27$ & $\mathrm{C} 22$ & $120.6(6)$ \\
$\mathrm{N} 3$ & $\mathrm{C} 23$ & $\mathrm{C} 24$ & $116.6(4)$ & & & & \\
\hline
\end{tabular}

Table S55. $\mathrm{IC}_{50}(\mu \mathrm{M})$ values of Co1-Co18 against five tested cells for $48 \mathrm{~h}$.

\begin{tabular}{|c|c|c|c|c|c|}
\hline Compd. & BEL-7404 & Hep-G2 & $\mathrm{HeLa}$ & MCF-7 & HL-7702 \\
\hline H-QL2 & $>150$ & $>150$ & $>150$ & $>150$ & $>100$ \\
\hline H-QL1 & $>100$ & $>100$ & $>100$ & $>100$ & $>100$ \\
\hline H-QL3 & $65.09 \pm 0.77$ & $58.63 \pm 1.41$ & $70.21 \pm 0.36$ & $50.22 \pm 1.99$ & $79.03 \pm 1.06$ \\
\hline H-QL4 & $>100$ & $80.11 \pm 0.41$ & $>100$ & $75.11 \pm 0.13$ & $71.02 \pm 1.53$ \\
\hline H-QL5 & $68.14 \pm 0.67$ & $>100$ & $83.21 \pm 1.03$ & $>100$ & $>100$ \\
\hline Co1 & $6.00 \pm 0.17$ & $8.36 \pm 1.06$ & $4.53 \pm 0.32$ & $10.52 \pm 1.22$ & $68.03 \pm 0.93$ \\
\hline $\mathrm{Co} 2$ & $9.02 \pm 0.44$ & $15.97 \pm 0.52$ & $7.21 \pm 1.03$ & $12.01 \pm 1.03$ & $85.02 \pm 0.45$ \\
\hline $\mathrm{Co3}$ & $4.01 \pm 1.09$ & $5.66 \pm 1.03$ & $1.99 \pm 0.79$ & $7.22 \pm 0.96$ & $71.25 \pm 0.22$ \\
\hline Co4 & $7.54 \pm 1.11$ & $11.03 \pm 1.99$ & $6.17 \pm 0.49$ & $10.01 \pm 0.46$ & $63.52 \pm 1.06$ \\
\hline $\mathrm{Co5}$ & $2.35 \pm 0.19$ & $3.69 \pm 1.58$ & $100.00 \pm 0.85 \mathrm{nM}$ & $5.16 \pm 0.25$ & $60.22 \pm 0.77$ \\
\hline Co6 & $4.09 \pm 1.88$ & $7.03 \pm 0.47$ & $5.28 \pm 0.14$ & $6.99 \pm 0.47$ & $62.14 \pm 1.09$ \\
\hline Co7 & $0.25 \pm 0.06$ & $0.12 \pm 0.15$ & $0.80 \pm 0.21 \mathrm{nM}$ & $0.94 \pm 1.03$ & $70.33 \pm 1.11$ \\
\hline $\mathrm{Co8}$ & $3.29 \pm 0.74$ & $4.12 \pm 0.63$ & $0.59 \pm 0.26$ & $5.67 \pm 0.21$ & $59.03 \pm 1.03$ \\
\hline Co9 & $5.34 \pm 0.95$ & $7.09 \pm 1.07$ & $3.06 \pm 0.39$ & $9.01 \pm 1.03$ & $70.24 \pm 0.63$ \\
\hline Co10 & $4.92 \pm 0.88$ & $6.21 \pm 1.64$ & $2.58 \pm 1.04$ & $7.95 \pm 0.88$ & $65.83 \pm 1.77$ \\
\hline Co11 & $3.86 \pm 1.09$ & $5.41 \pm 1.07$ & $1.09 \pm 0.19$ & $6.18 \pm 0.45$ & $65.52 \pm 0.45$ \\
\hline
\end{tabular}




\begin{tabular}{cccccc} 
Co12 & $10.31 \pm 0.84$ & $23.63 \pm 1.54$ & $8.47 \pm 1.29$ & $15.11 \pm 1.88$ & $63.89 \pm 0.49$ \\
Co13 & $5.94 \pm 1.53$ & $8.00 \pm 0.79$ & $4.11 \pm 1.25$ & $10.26 \pm 0.49$ & $80.11 \pm 1.06$ \\
Co14 & $31.52 \pm 1.17$ & $40.09 \pm 1.05$ & $10.62 \pm 0.39$ & $27.01 \pm 1.23$ & $55.02 \pm 1.74$ \\
Co15 & $19.46 \pm 1.09$ & $33.74 \pm 1.06$ & $9.54 \pm 0.84$ & $23.53 \pm 2.01$ & $75.85 \pm 1.15$ \\
Co16 & $35.69 \pm 0.88$ & $46.29 \pm 1.58$ & $11.88 \pm 0.56$ & $32.98 \pm 0.49$ & $80.45 \pm 0.33$ \\
Co17 & $25.01 \pm 0.44$ & $38.09 \pm 0.14$ & $10.07 \pm 0.21$ & $25.99 \pm 1.66$ & $64.56 \pm 0.79$ \\
Co18 & $15.05 \pm 0.71$ & $30.54 \pm 0.96$ & $9.22 \pm 0.59$ & $20.09 \pm 0.55$ & $60.21 \pm 1.55$ \\
py & $>50$ & $>50$ & $>50$ & $>50$ & $>50$ \\
Phen & $>50$ & $>50$ & $>50$ & $>50$ & $>50$ \\
DPQ & $>50$ & $>50$ & $>50$ & $>50$ & $>50$ \\
DPPZ & $>50$ & $>50$ & $>50$ & $>50$ & $>50$ \\
MDP & $>50$ & $>50$ & $>50$ & $>50$ & $>50$ \\
ODP & $>50$ & $>50$ & $>50$ & $>50$ & $>50$ \\
PPT & $>50$ & $>50$ & $>50$ & $>50$ & $>50$ \\
ClPT & $>50$ & $>50$ & $>50$ & $>50$ & $>50$ \\
dpy & $>50$ & $>50$ & $>50$ & $>50$ & $>50$ \\
mpy & $>50$ & $>50$ & $>50$ & $>50$ & $>50$ \\
$\mathrm{CoCl}_{2} \cdot 6 \mathrm{H}_{2} \mathrm{O}$ & $>150$ & $>150$ & $>150$ & $>150$ & $>150$ \\
cisplatin & $13.06 \pm 1.01$ & $14.02 \pm 1.41$ & $17.03 \pm 1.05$ & $14.18 \pm 1.03$ & $17.06 \pm 1.04$ \\
\hline
\end{tabular}

Table S56. Cellular distribution of Co1 $(4.53 \mu \mathrm{M})$ and Co7 $(0.80 \mathrm{nM})$ in HeLa cells after $24.0 \mathrm{~h}$ of incubation.

\begin{tabular}{cccc}
\hline Co1 & $(18.06 \pm 0.05 \mathrm{nmol}$ of Co $) / 10^{6}$ & $(1.06 \pm 0.02 \mathrm{nmol}$ of & $(7.25 \pm 0.12 \mathrm{nmol}$ \\
& cells & Co $) / 10^{6}$ cells & of Co $) / 10^{6}$ cells \\
Co7 & $(22.96 \pm 0.15 \mathrm{nmol}$ of Co $) / 10^{6}$ & $(7.11 \pm 0.03 \mathrm{nmol}$ of & $(7.93 \pm 0.17 \mathrm{nmol}$ \\
& cells & Co $) / 10^{6}$ cells & of Co $) / 10^{6}$ cells \\
\hline
\end{tabular}




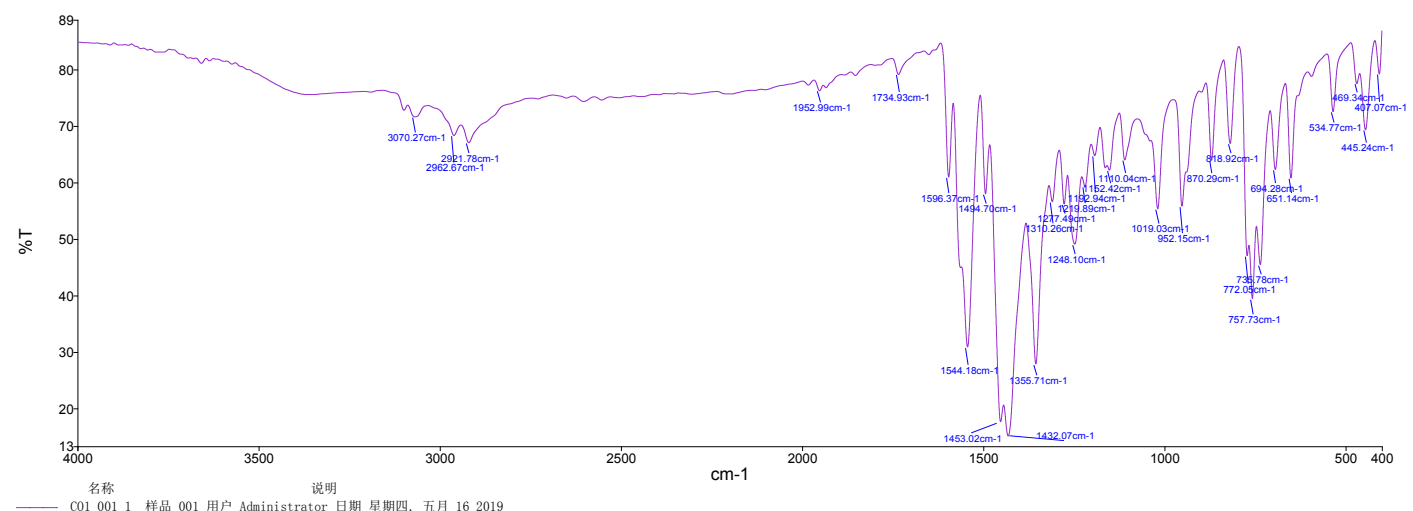

Fig. S1. IR (KBr) spectra of Co1

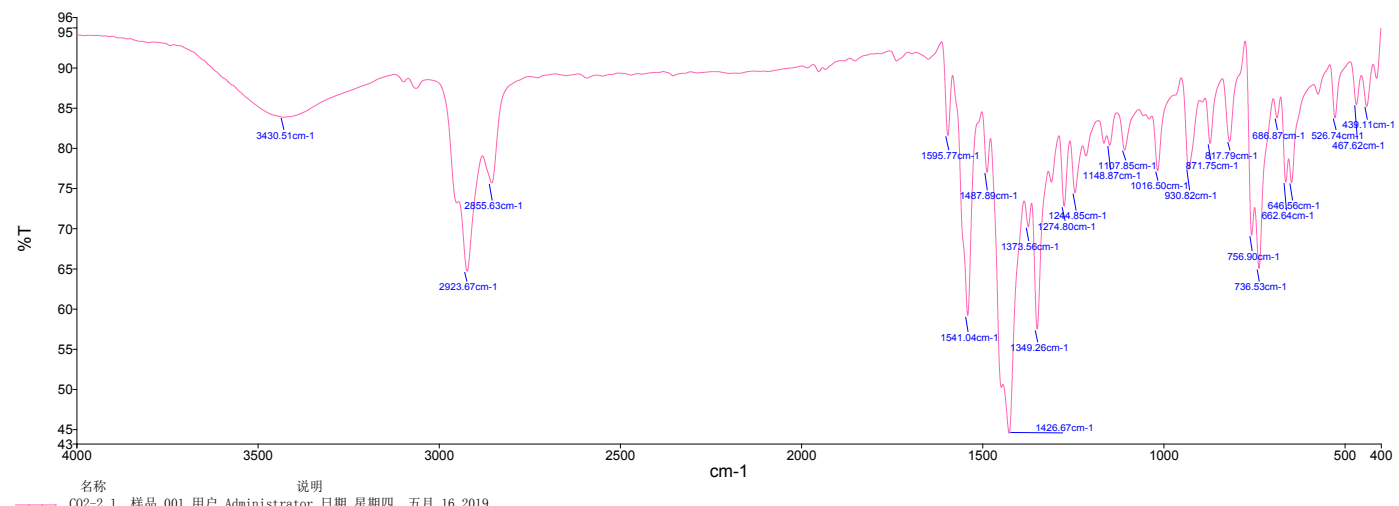

Fig. S2. IR ( $\mathrm{KBr}$ ) spectra of $\mathbf{C o 2}$

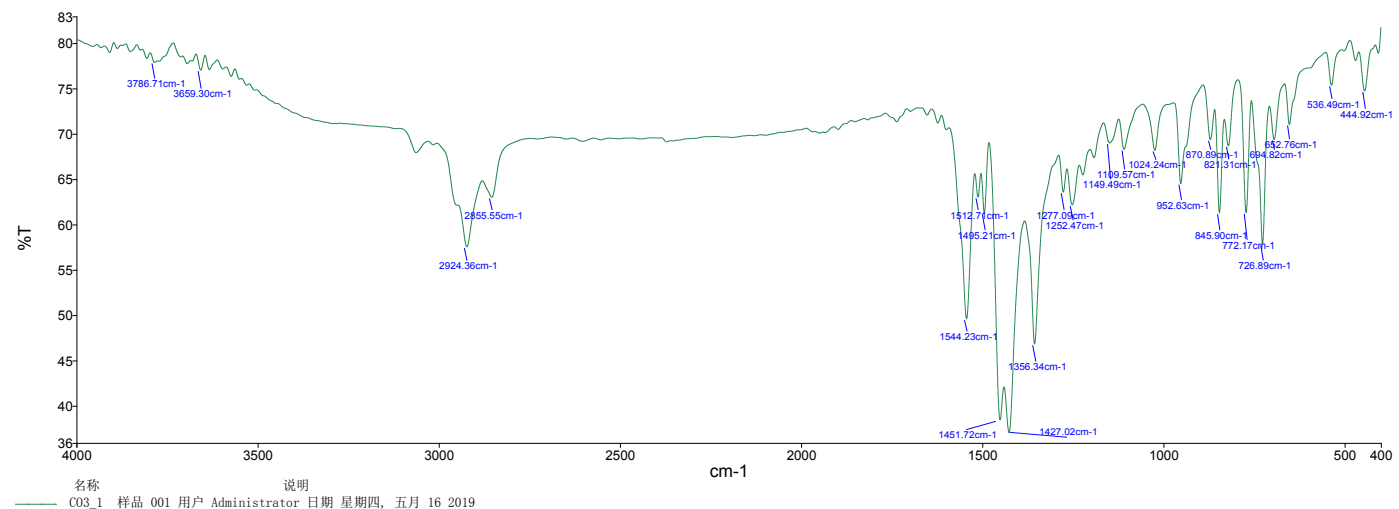

Fig. S3. IR (KBr) spectra of $\mathbf{C o 3}$ 


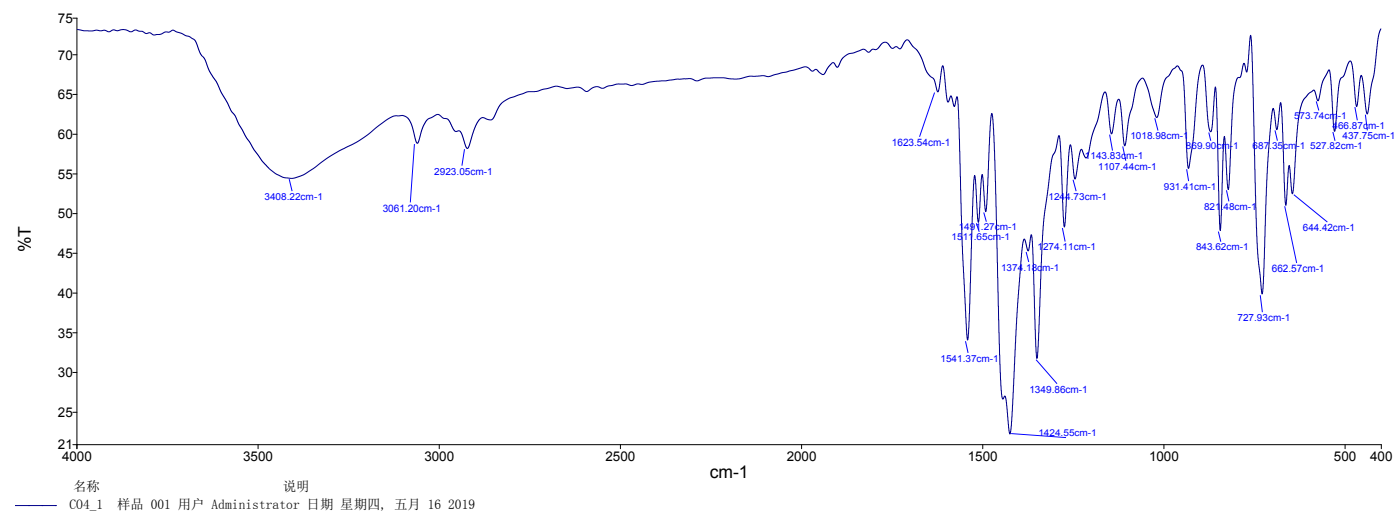

Fig. S4. IR (KBr) spectra of $\mathrm{Co4}$

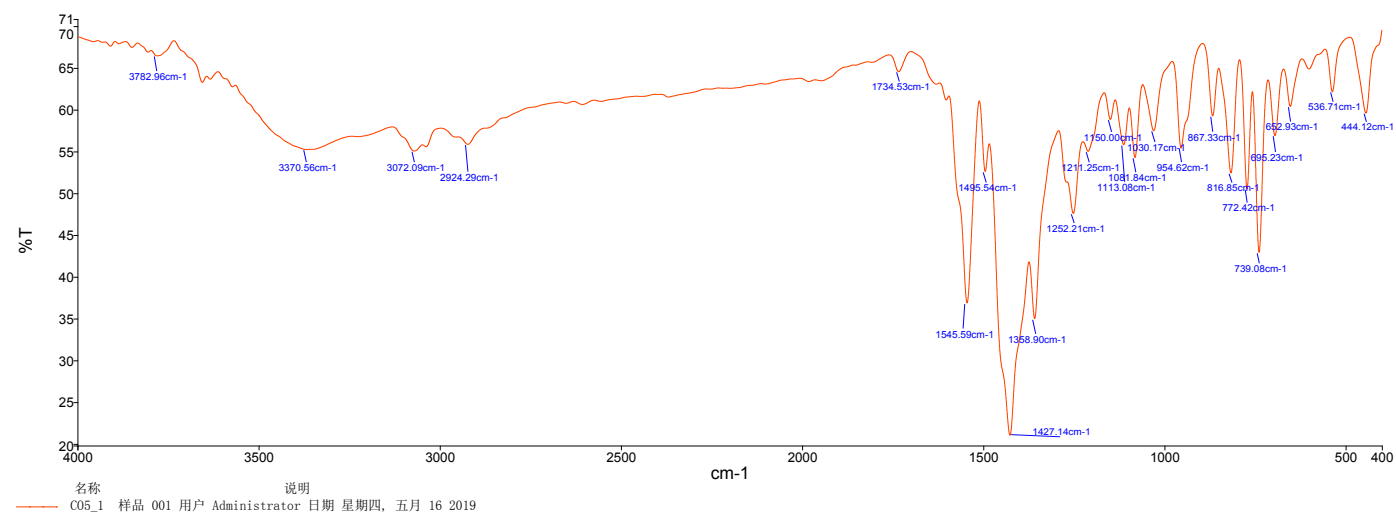

Fig. S5. IR (KBr) spectra of $\mathbf{C o 5}$

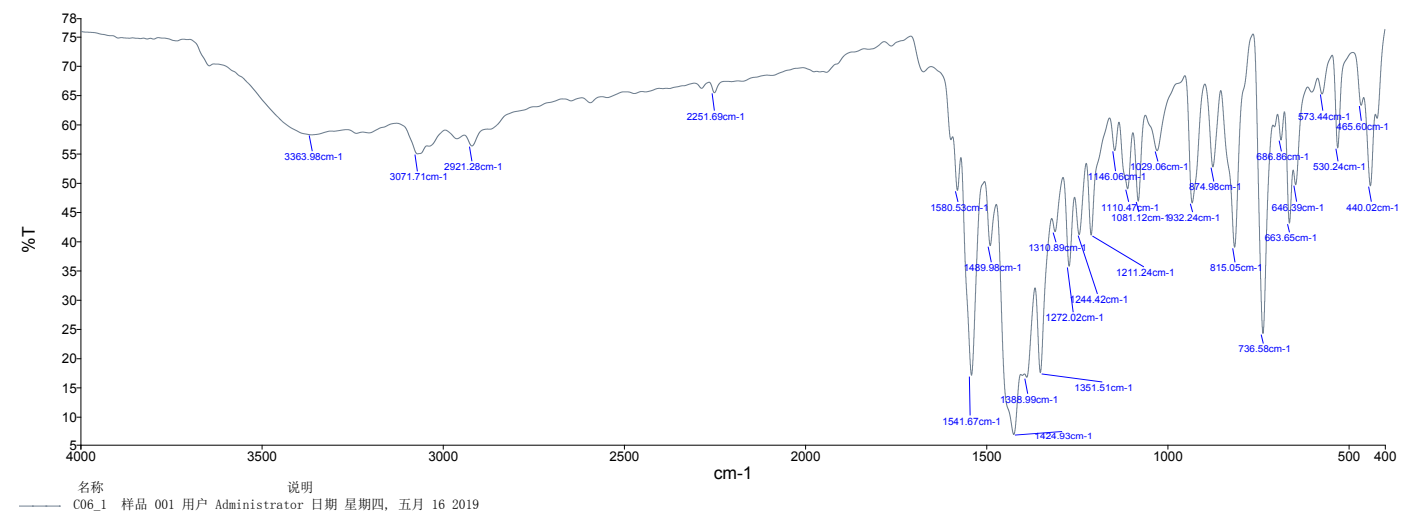

Fig. S6. IR (KBr) spectra of Co6 


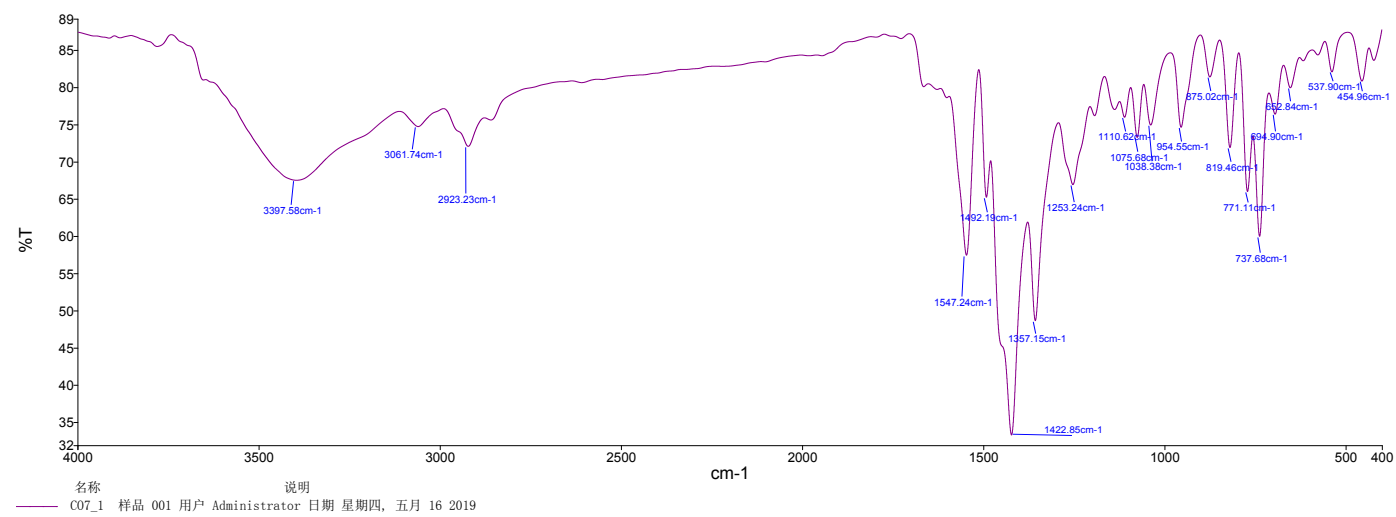

Fig. S7. IR (KBr) spectra of $\mathbf{C o} 7$

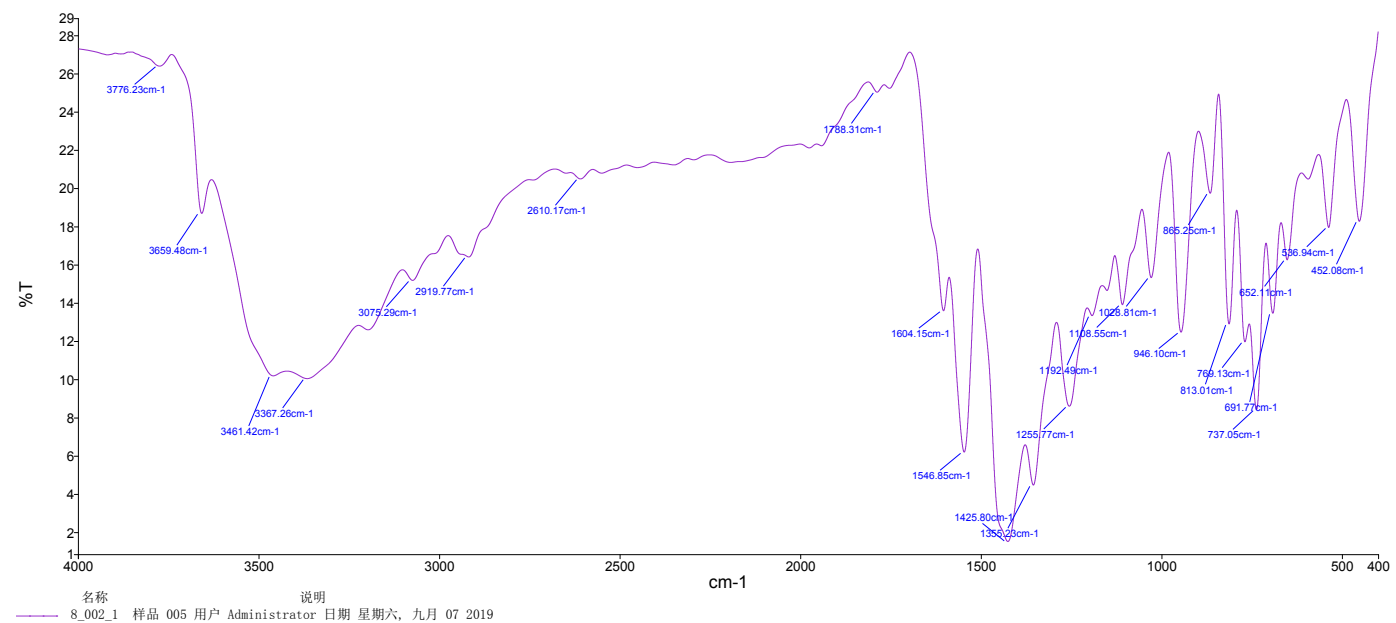

Fig. S8. IR (KBr) spectra of Co8

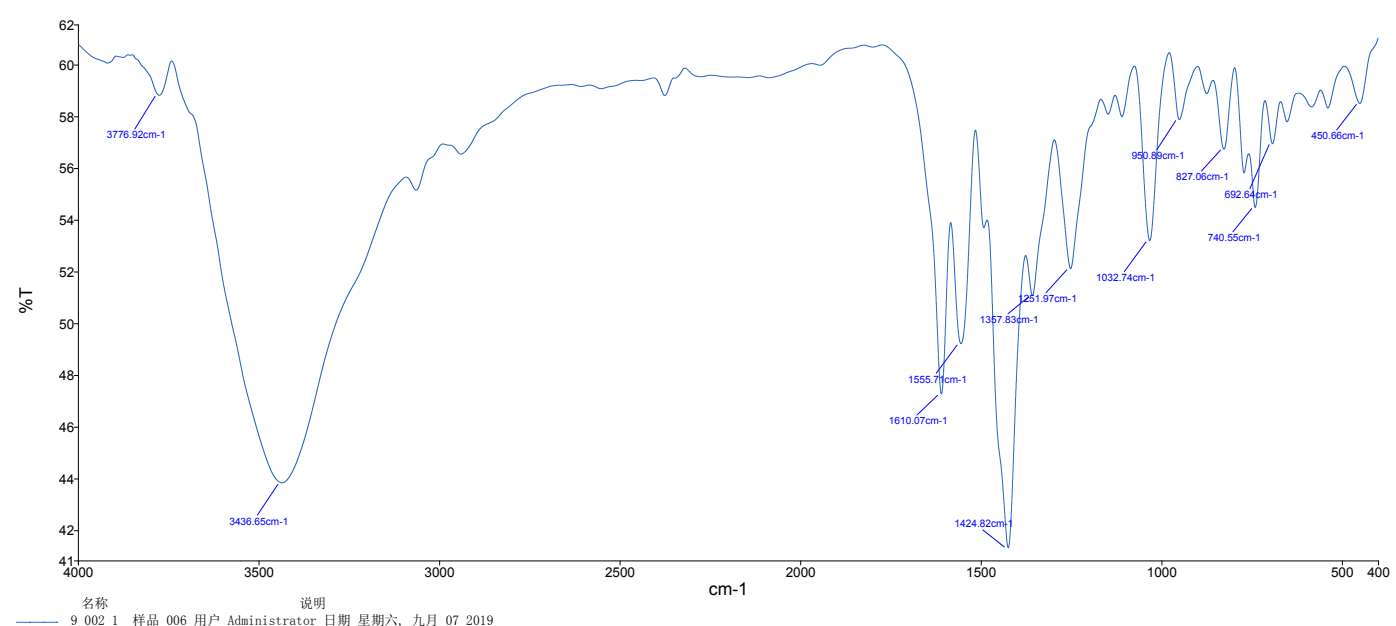

Fig. S9. IR (KBr) spectra of Co9 


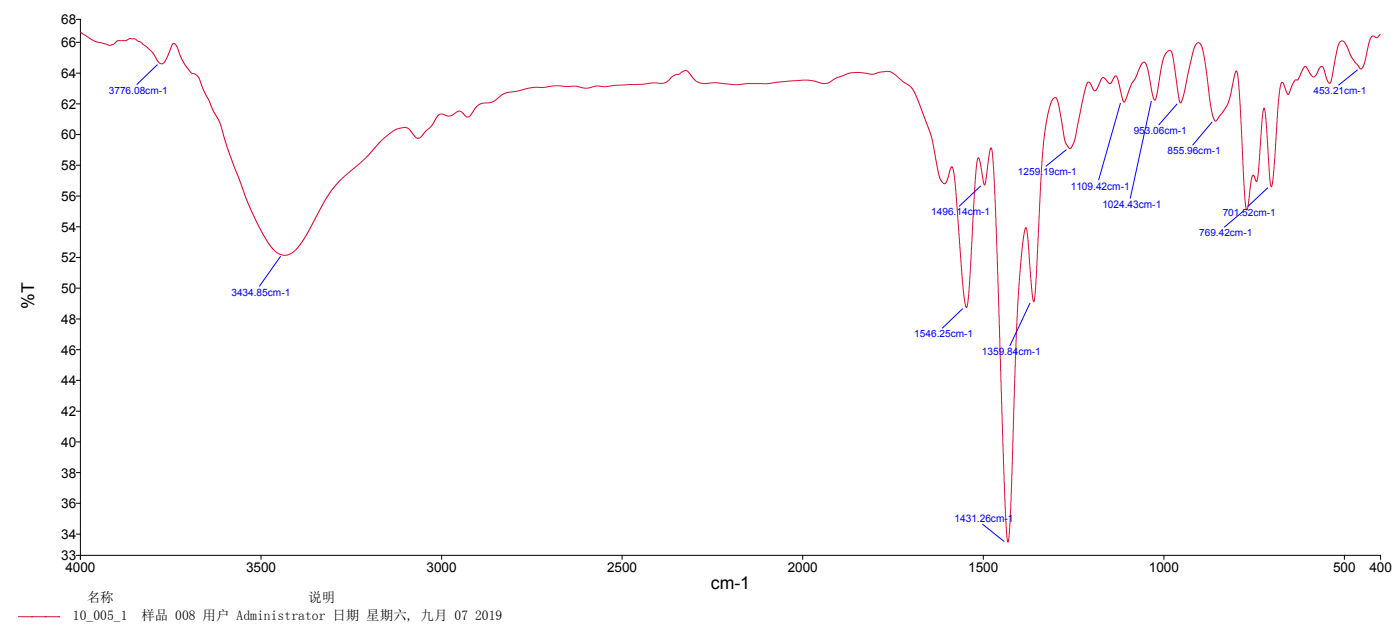

Fig. S10. IR (KBr) spectra of Co10

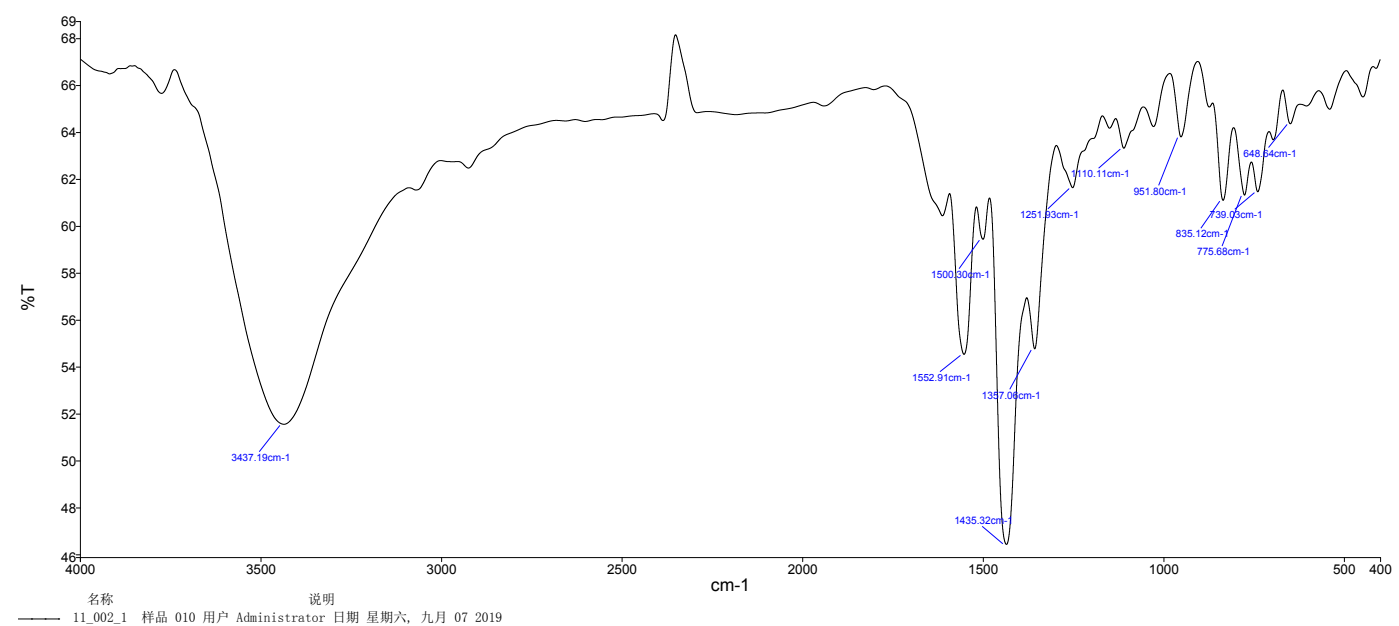

Fig. S11. IR (KBr) spectra of Co11

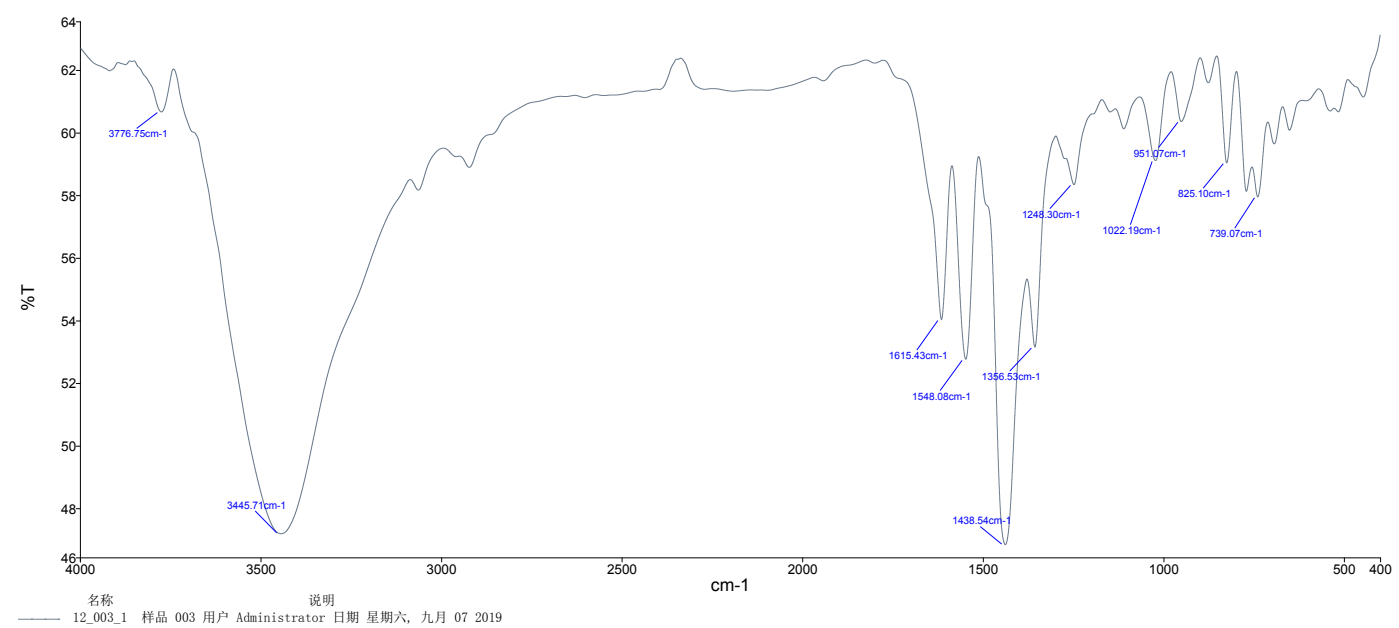

Fig. S12. IR (KBr) spectra of Co12 


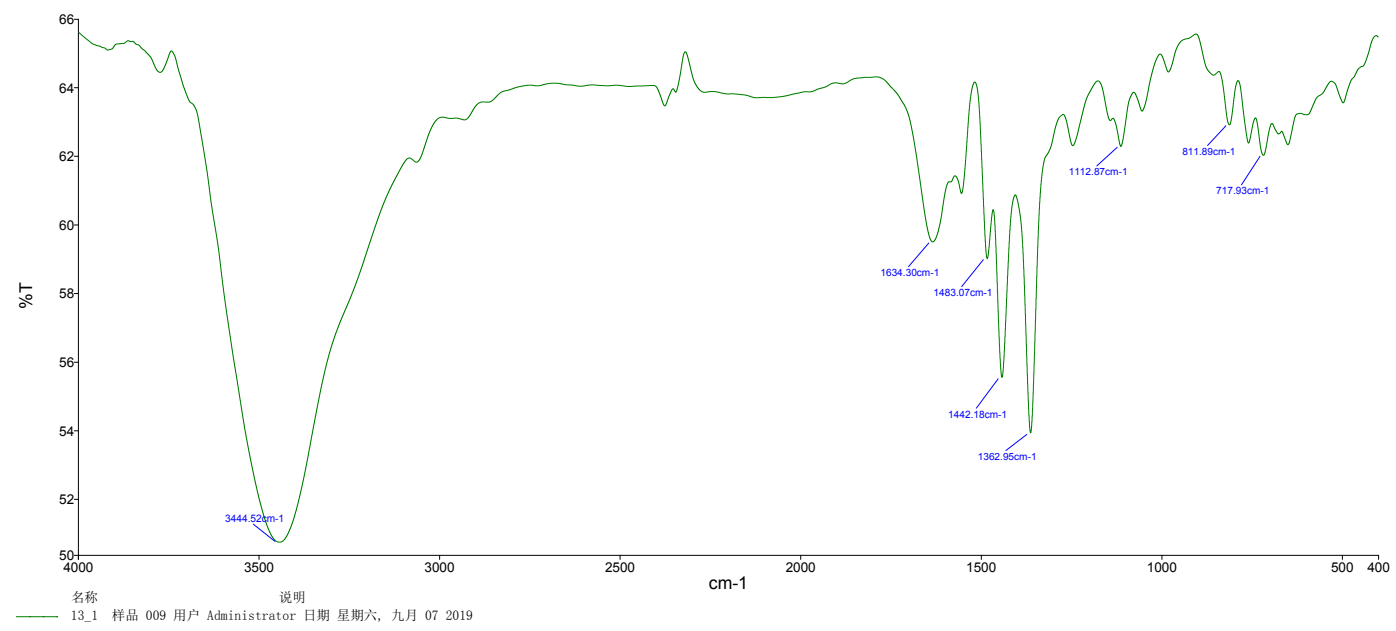

Fig. S13. IR (KBr) spectra of Co13

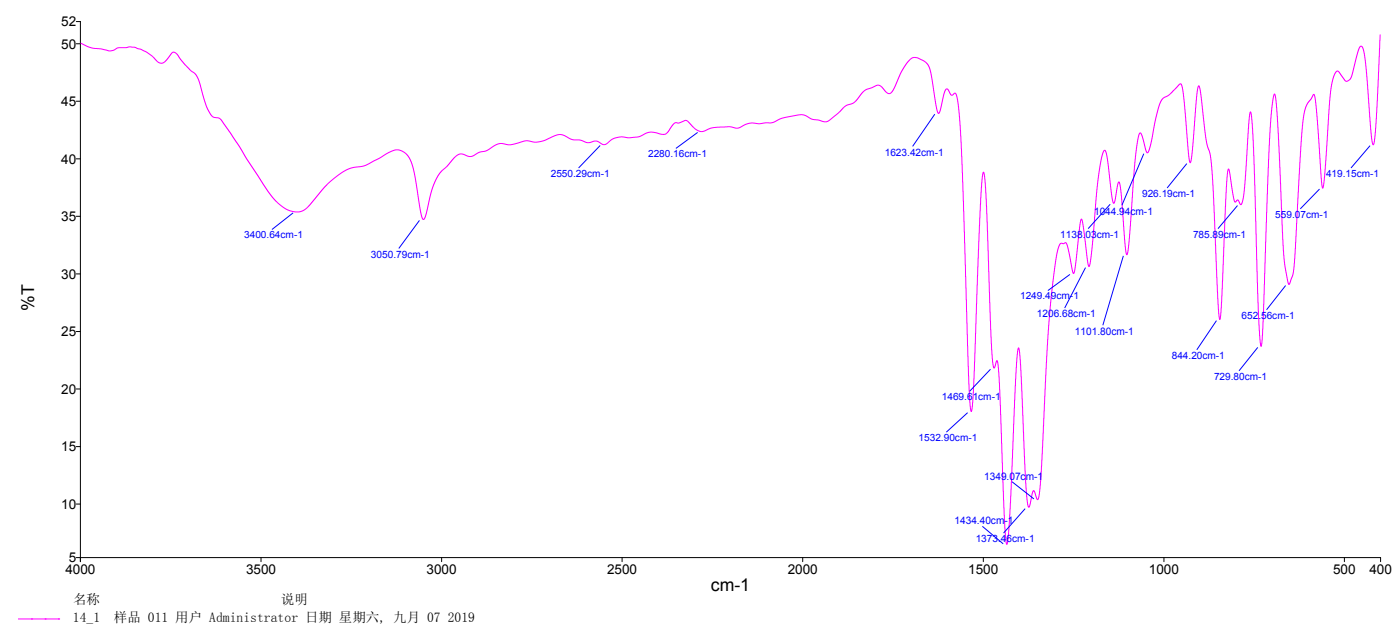

Fig. S14. IR (KBr) spectra of Co14

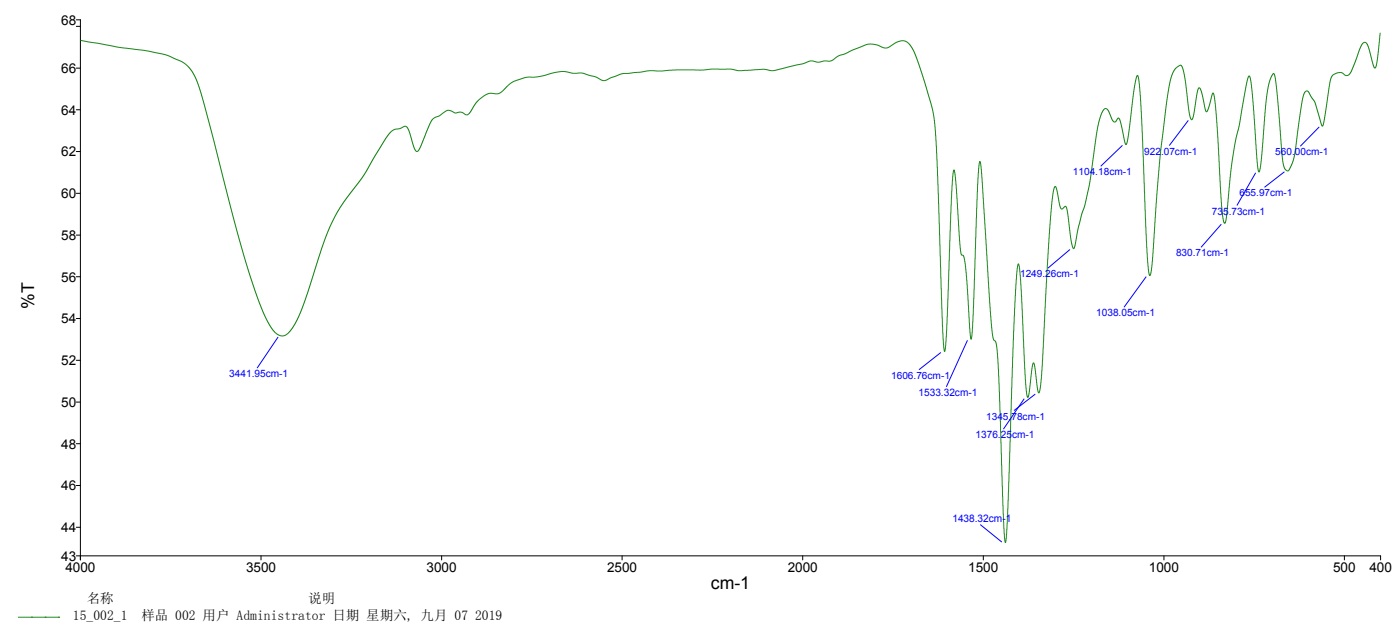

Fig. S15. IR ( $\mathrm{KBr}$ ) spectra of Co15 


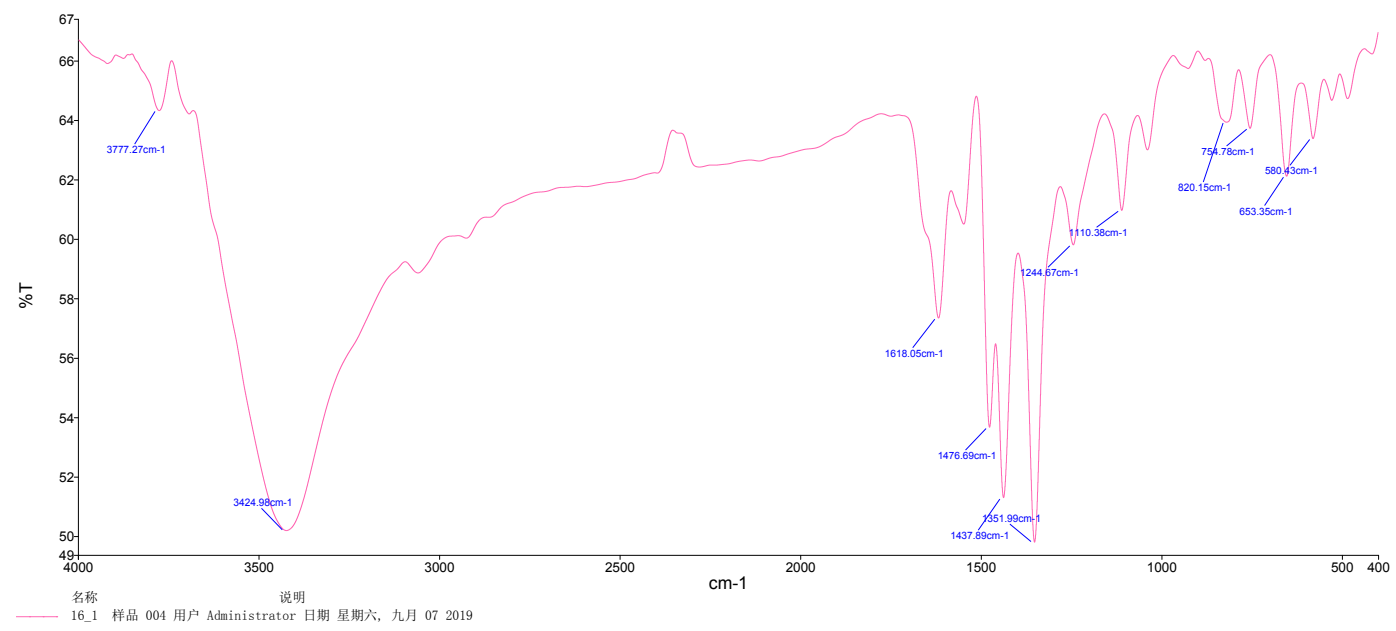

Fig. S16. IR (KBr) spectra of Co16

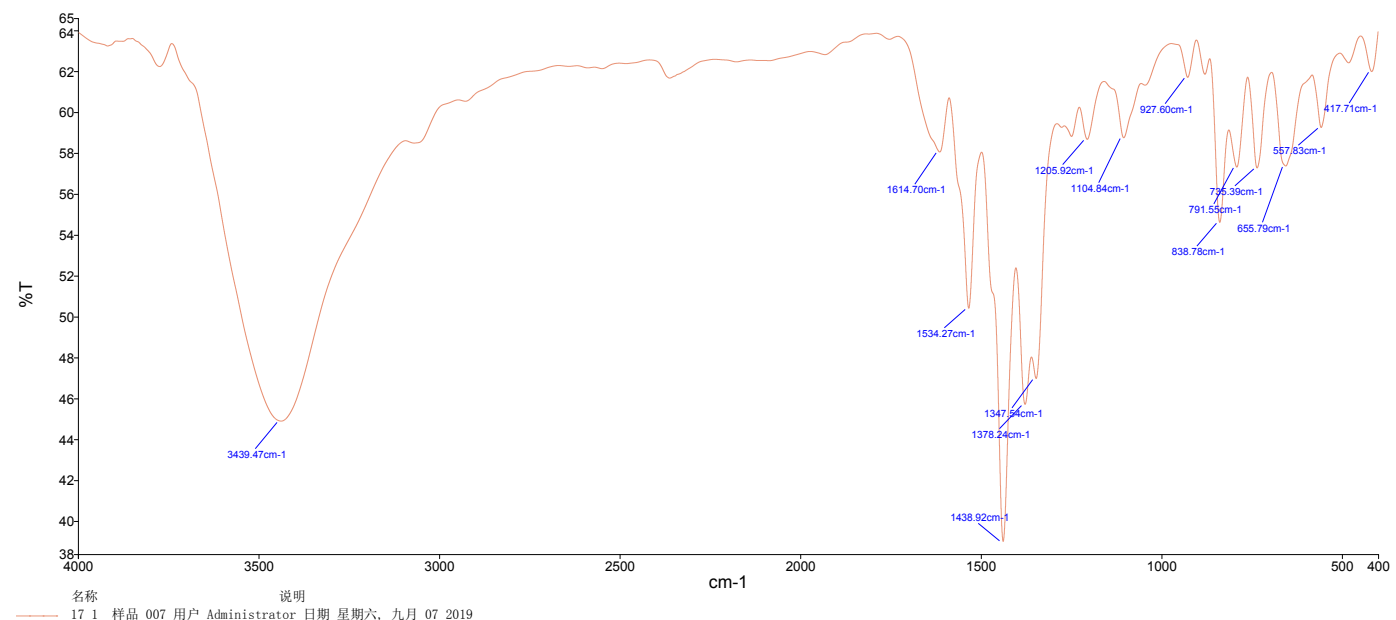

Fig. S17. IR (KBr) spectra of Co17

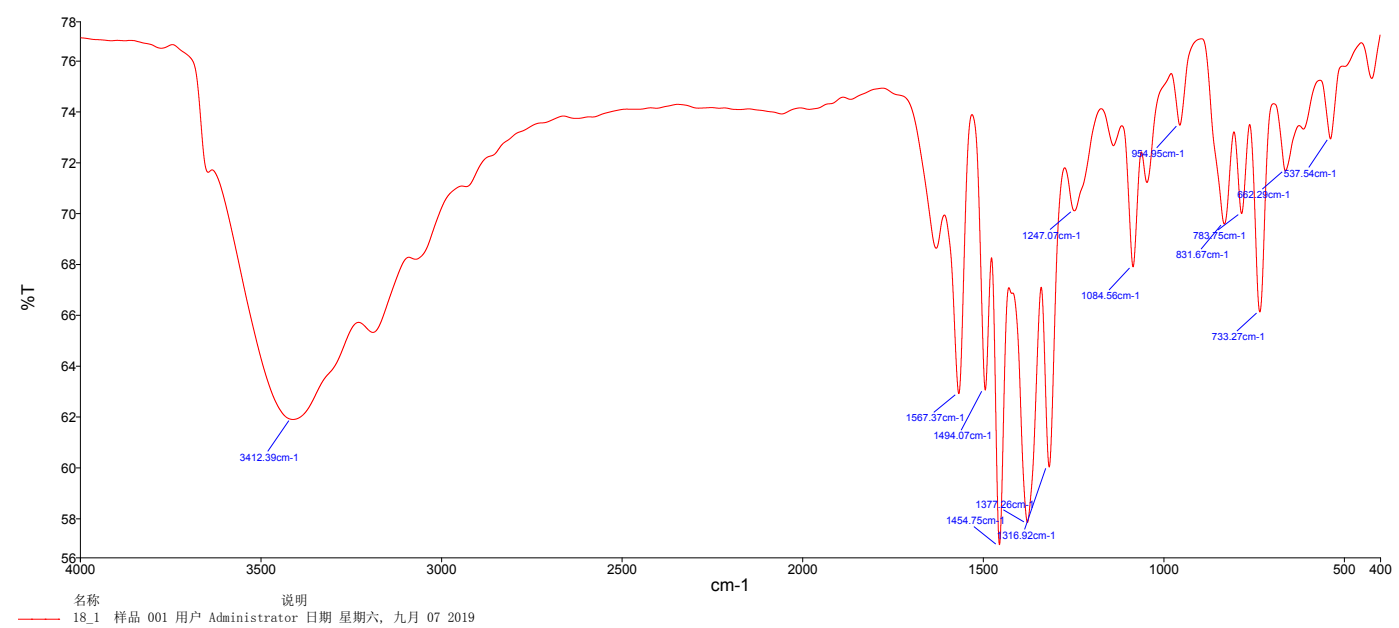

Fig. S18. IR ( $\mathrm{KBr})$ spectra of Co18 


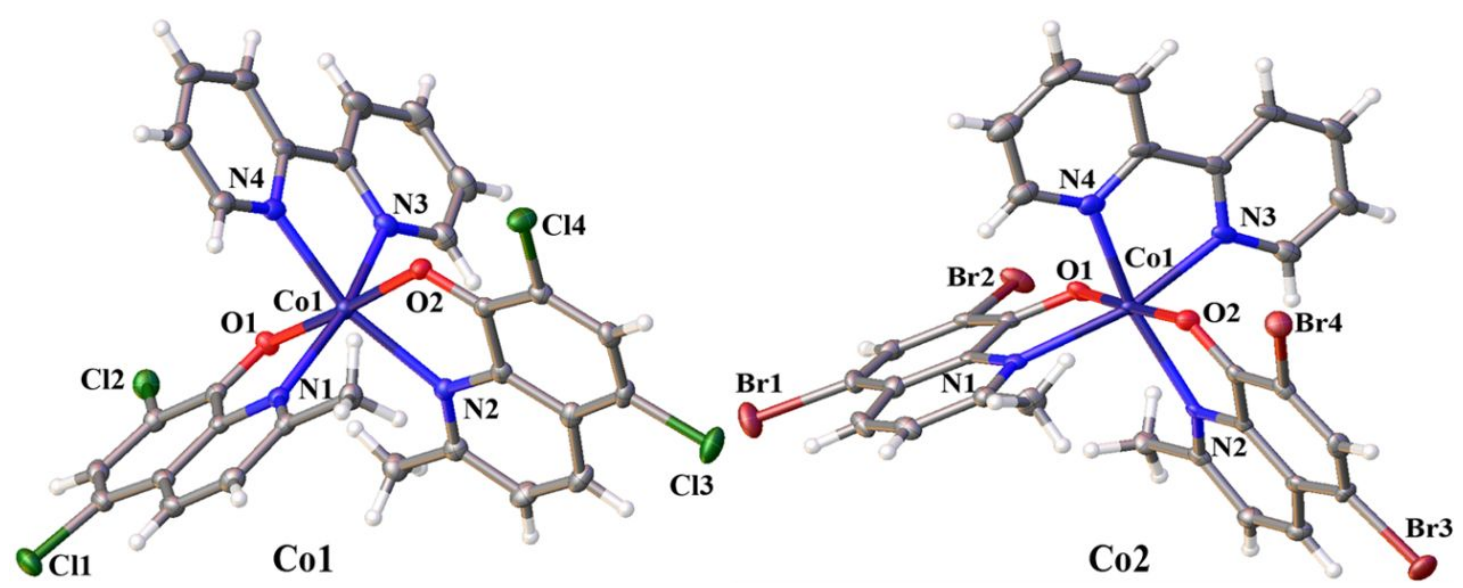

Fig. S19. ORTEP view of Co1 and Co2.
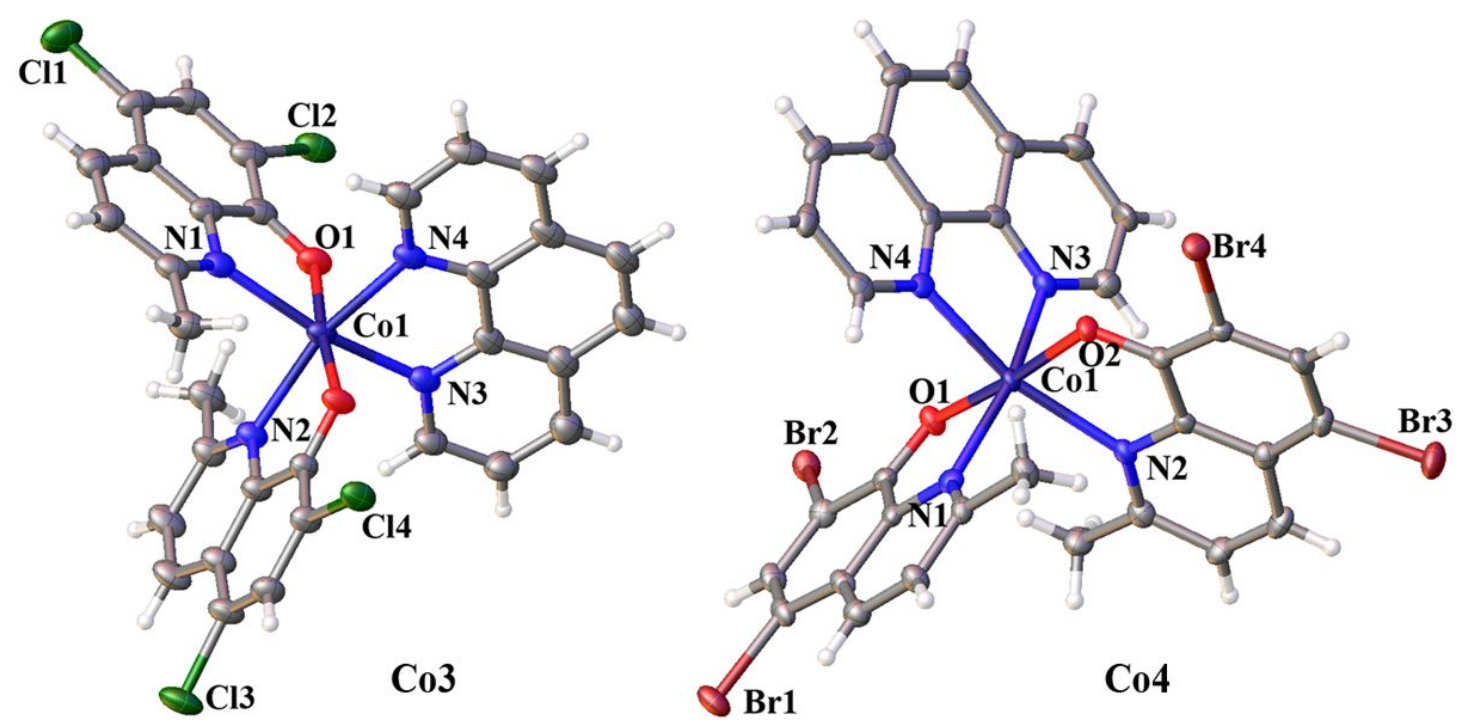

Fig. S20. ORTEP view of $\mathrm{Co3}$ and Co4.
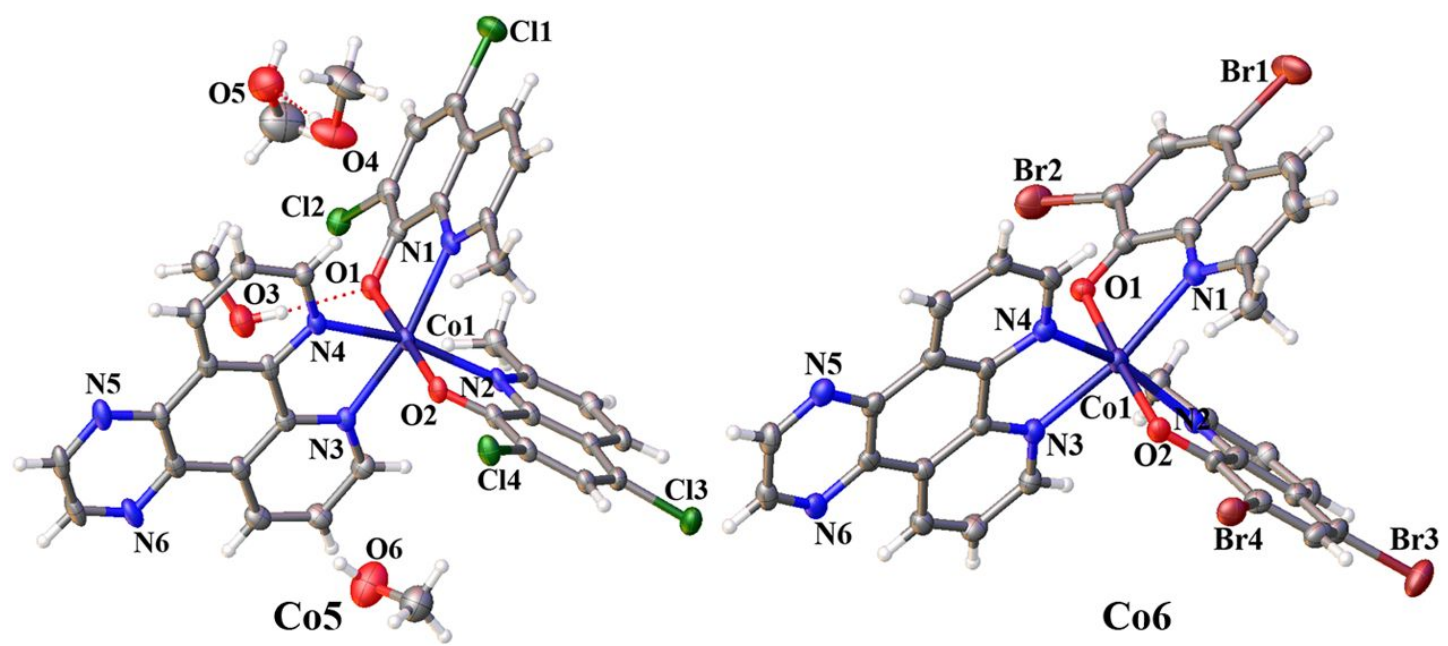

Fig. S21. ORTEP view of $\mathbf{C o 5}$ and Co6. 

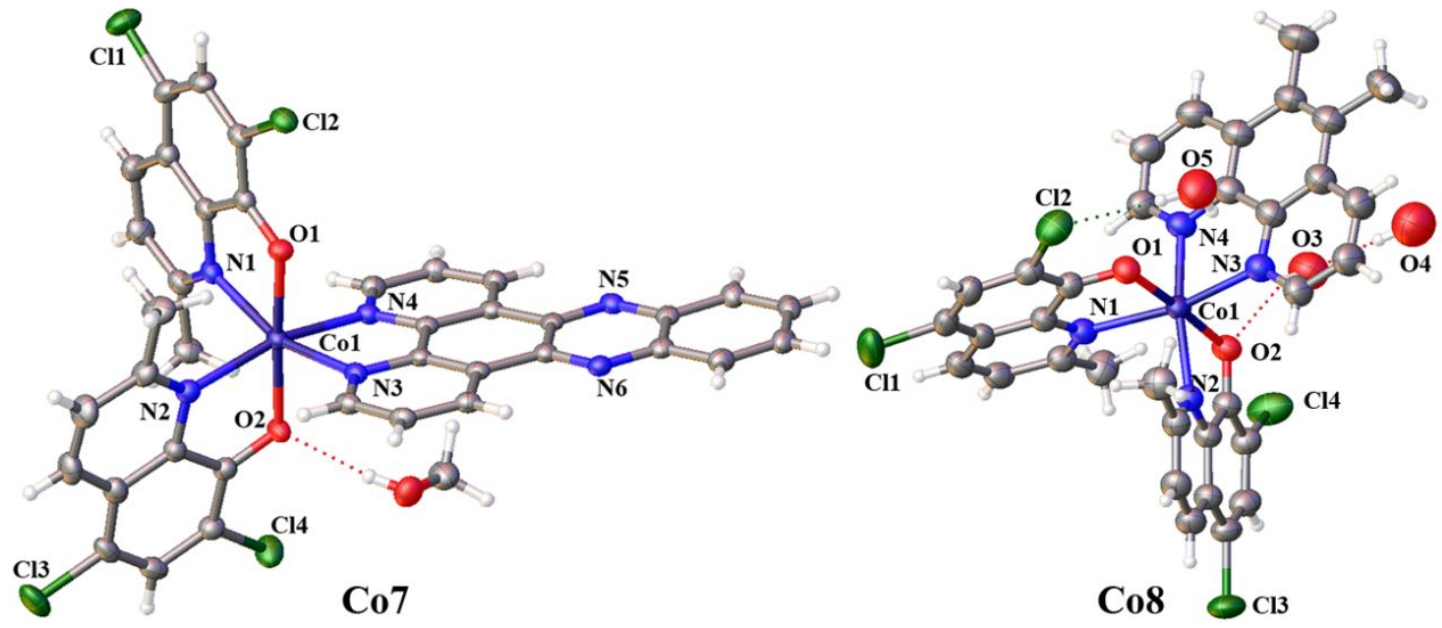

Fig. S22. ORTEP view of $\mathbf{C o} 7$ and $\mathbf{C o 8}$.
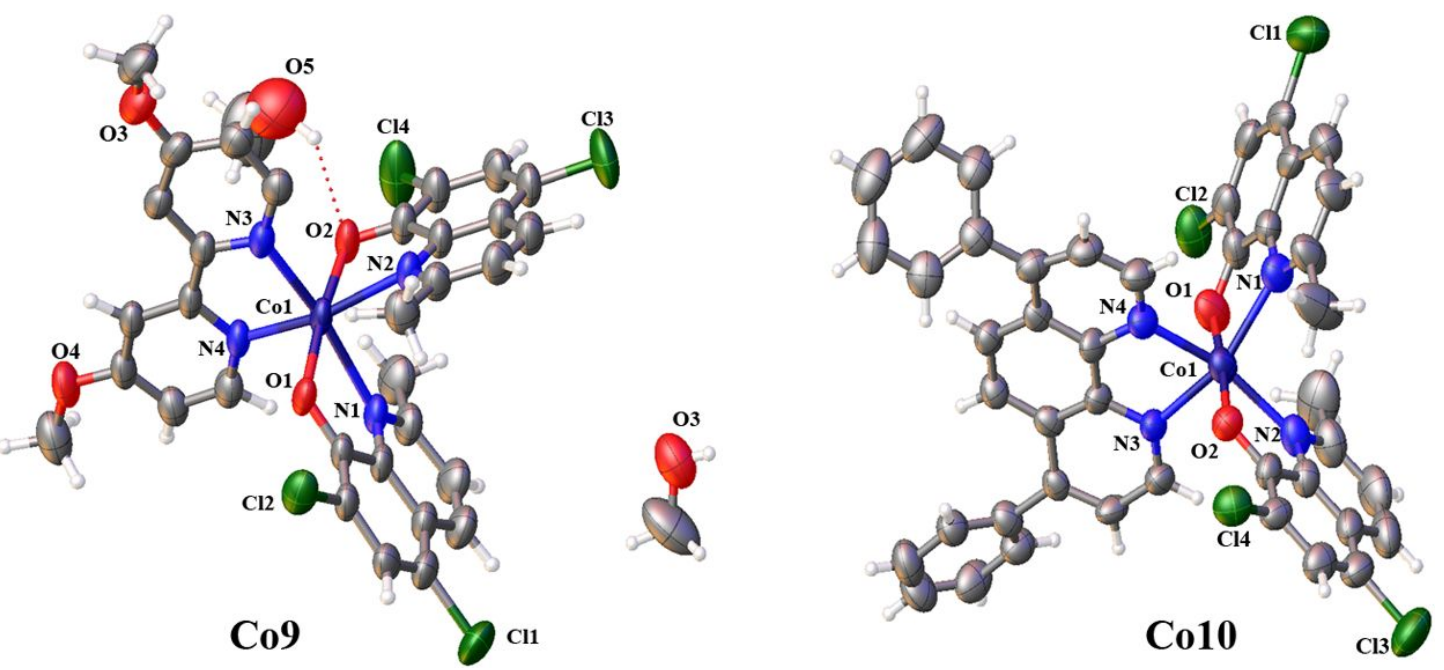

Fig. S23. ORTEP view of Co9 and Co10.

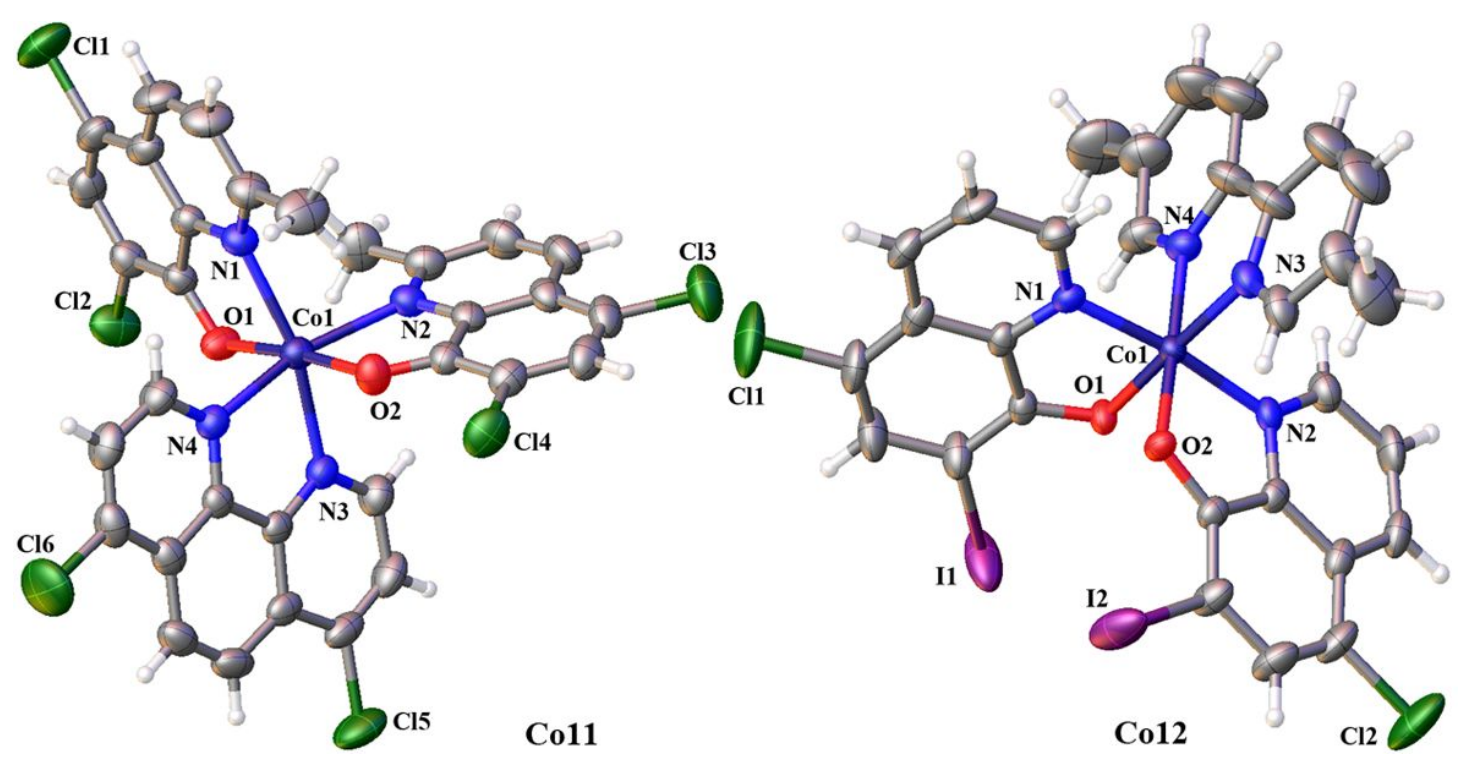

Fig. S24. ORTEP view of Co11 and Co12. 

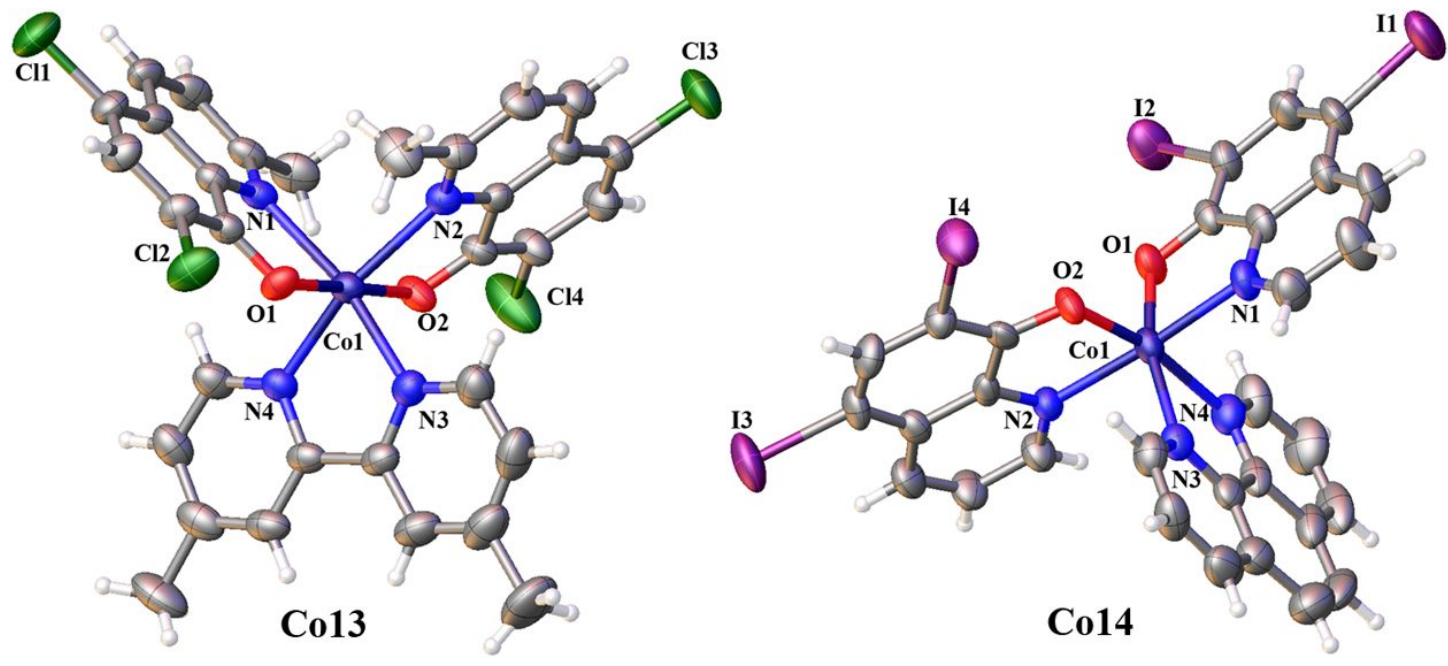

Fig. S25. ORTEP view of Co13 and Co14.
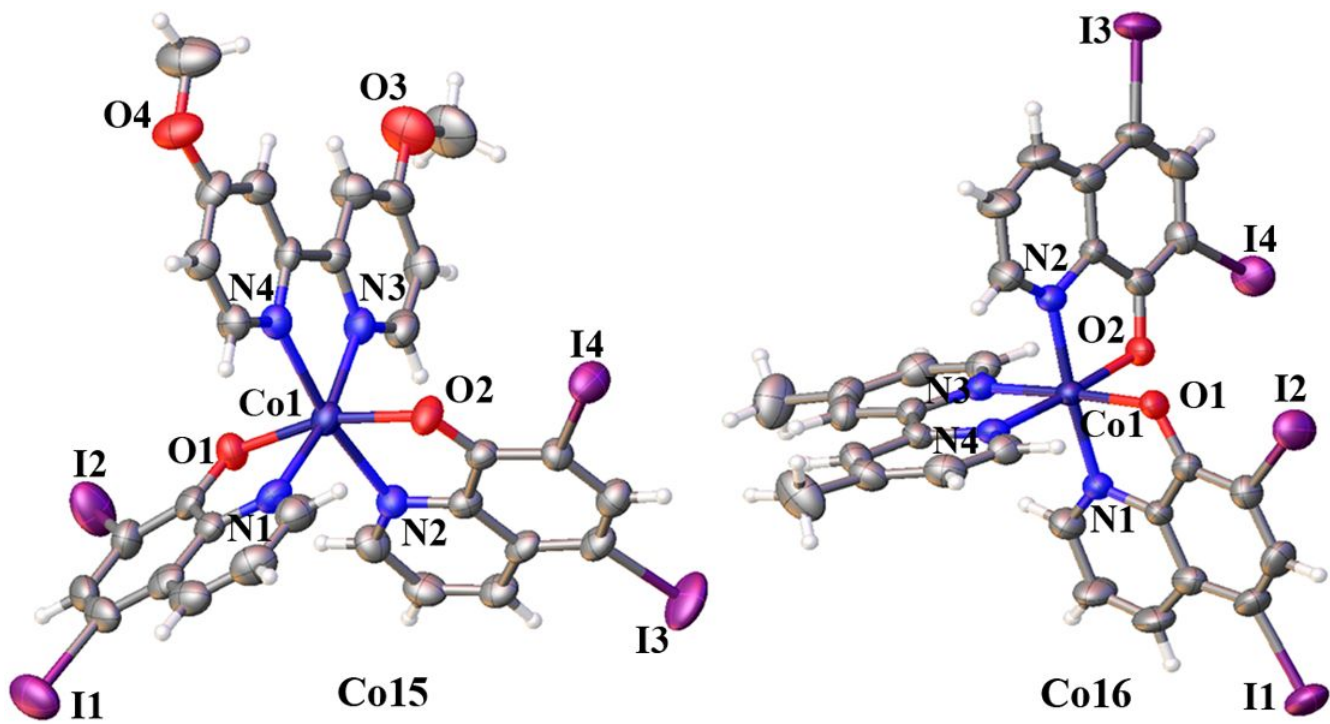

Fig. S26. ORTEP view of Co15 and Co16. 


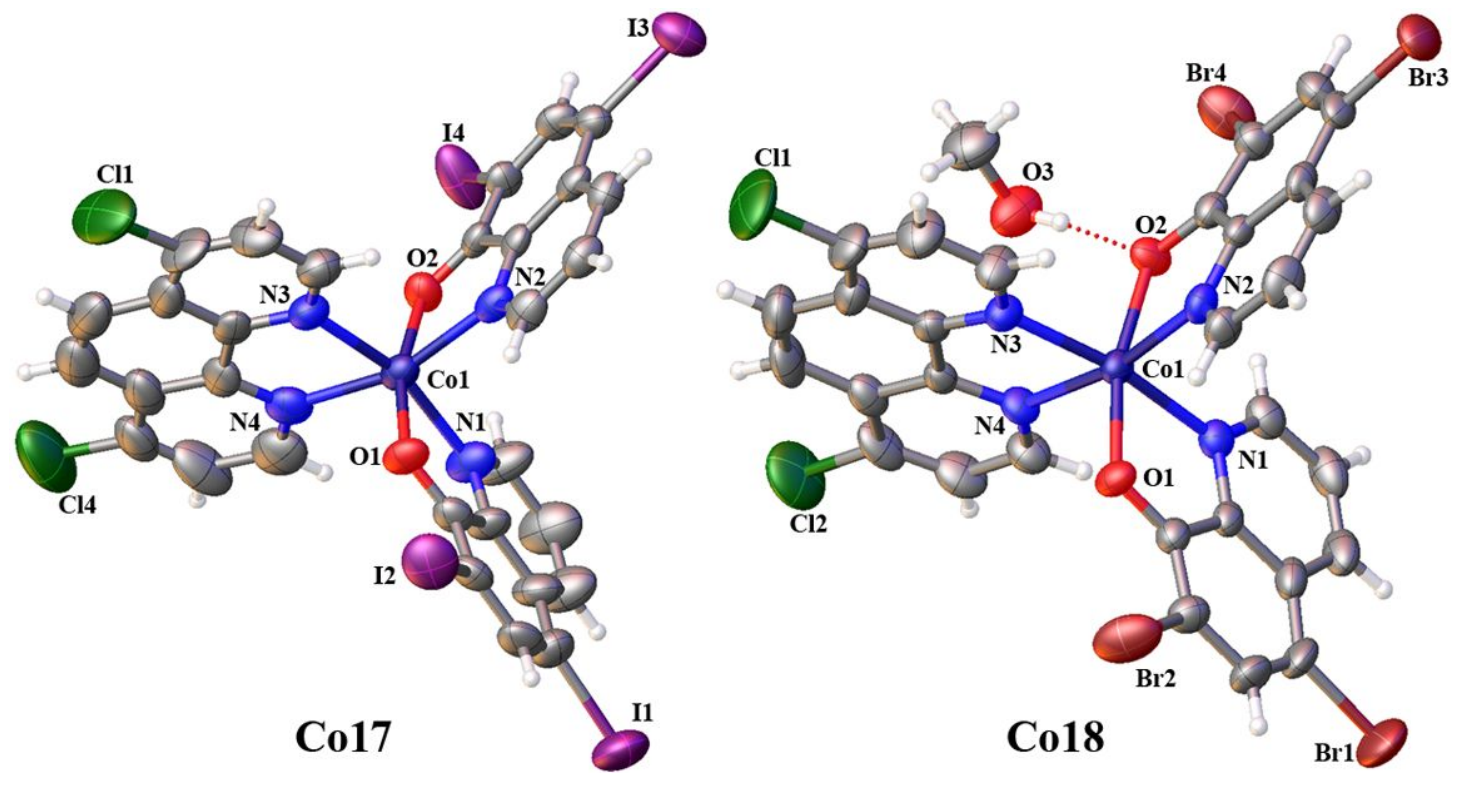

Fig. S27. ORTEP view of Co17 and Co18.

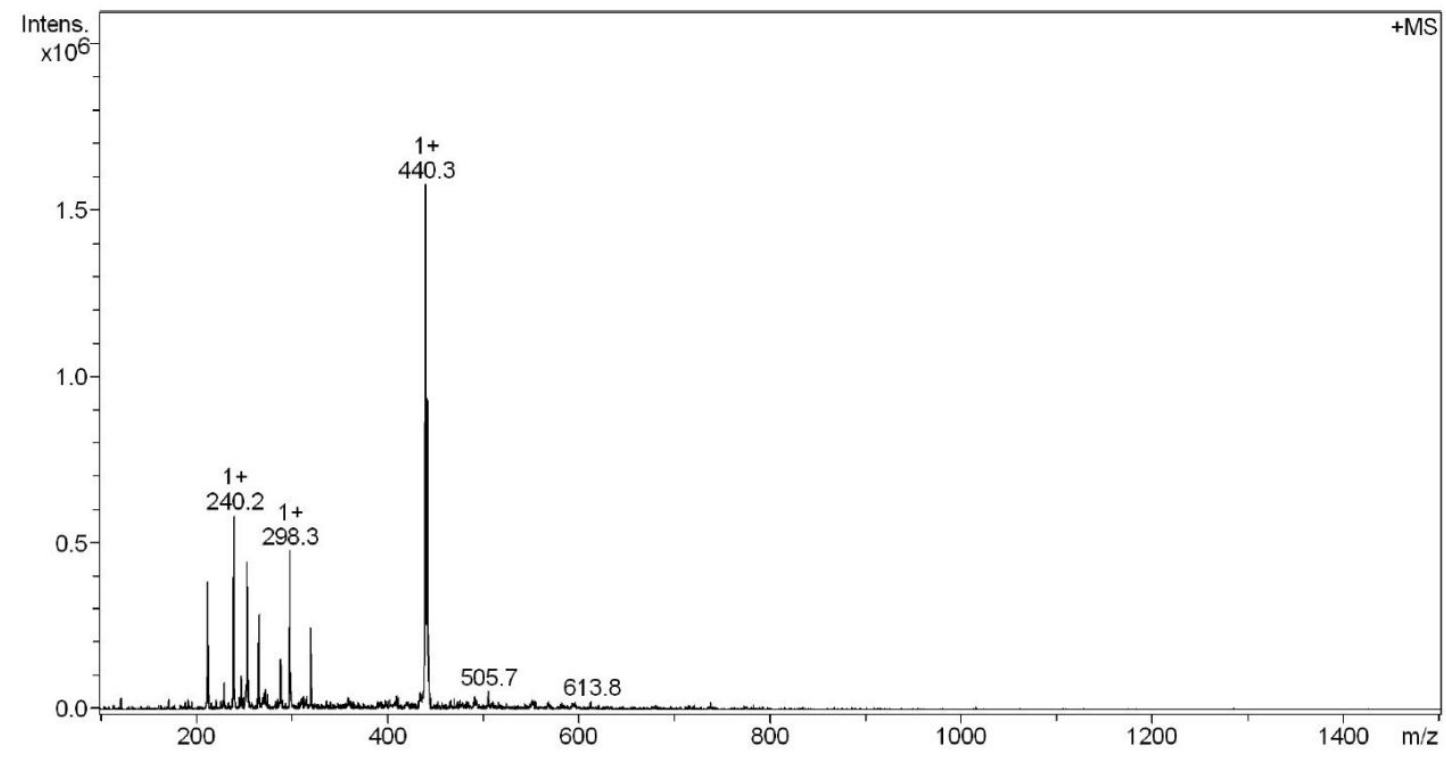

Figure S28. The mass spectra of $\mathbf{C o 1}\left(3.0 \times 10^{-5} \mathrm{M}\right)$ in Tris- $\mathrm{HCl}$ buffer solution (containing 5\% DMSO) for $0 \mathrm{~h}$. 


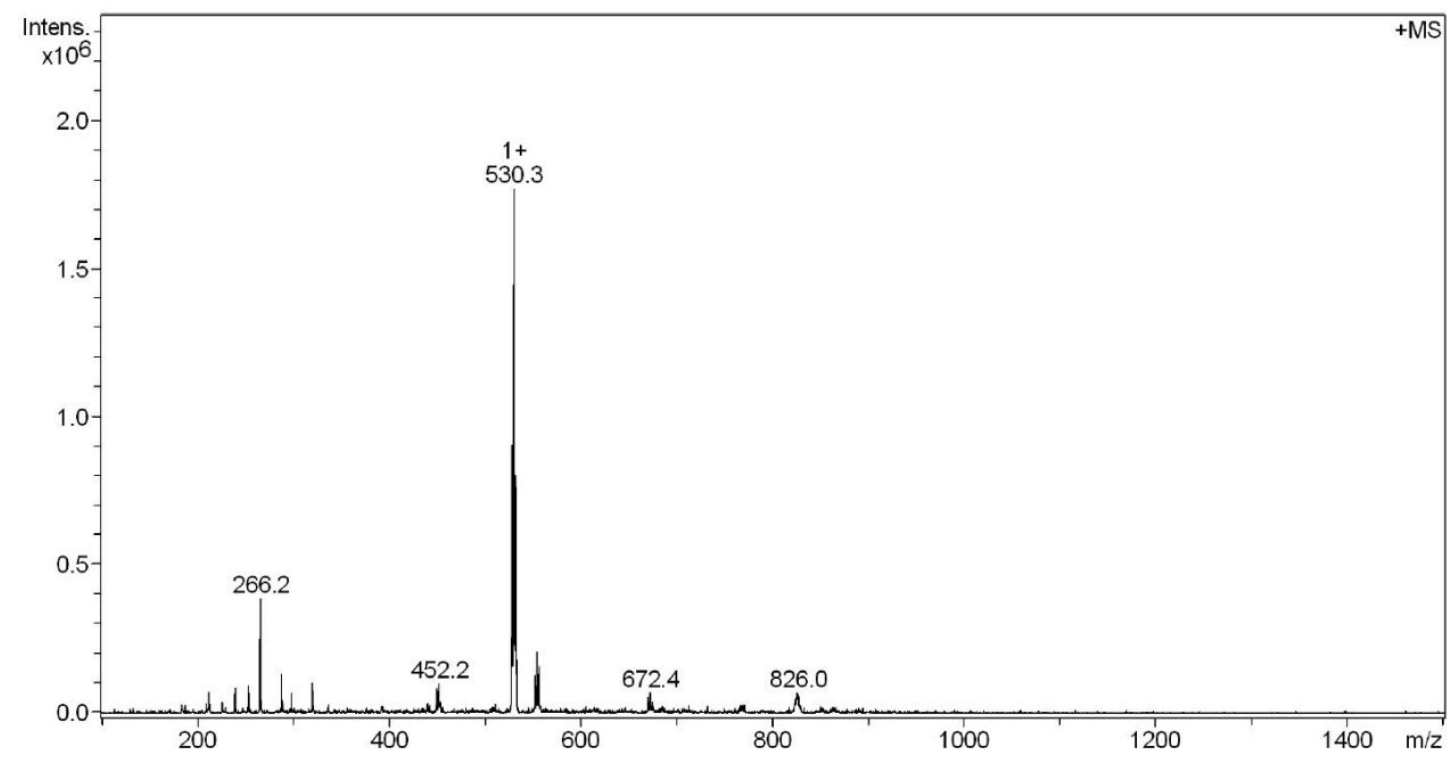

Figure S29. The mass spectra of $\mathbf{C o 2}\left(3.0 \times 10^{-5} \mathrm{M}\right)$ in Tris- $\mathrm{HCl}$ buffer solution (containing 5\% DMSO) for $0 \mathrm{~h}$.

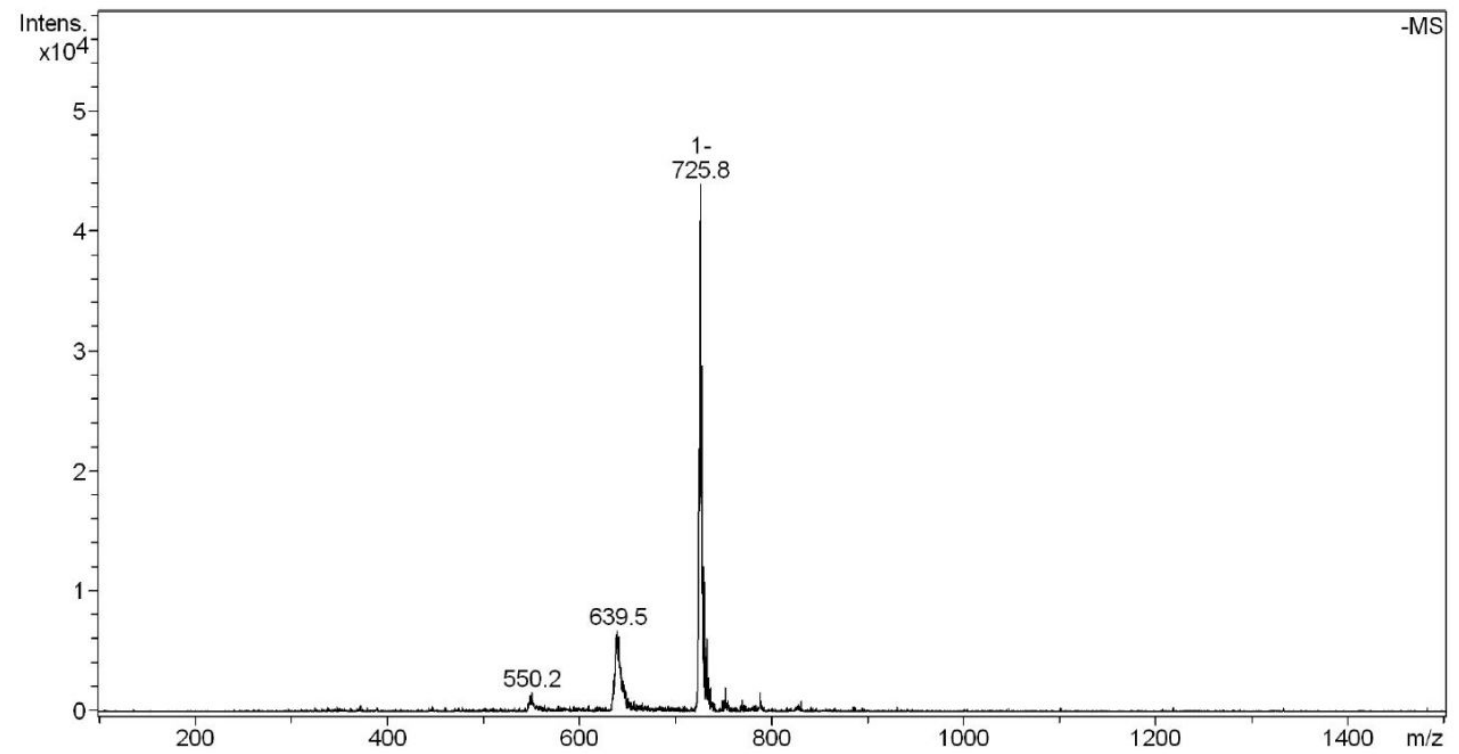

Figure S30. The mass spectra of $\mathbf{C o 3}\left(3.0 \times 10^{-5} \mathrm{M}\right)$ in Tris- $\mathrm{HCl}$ buffer solution (containing 5\% DMSO) for $0 \mathrm{~h}$. 


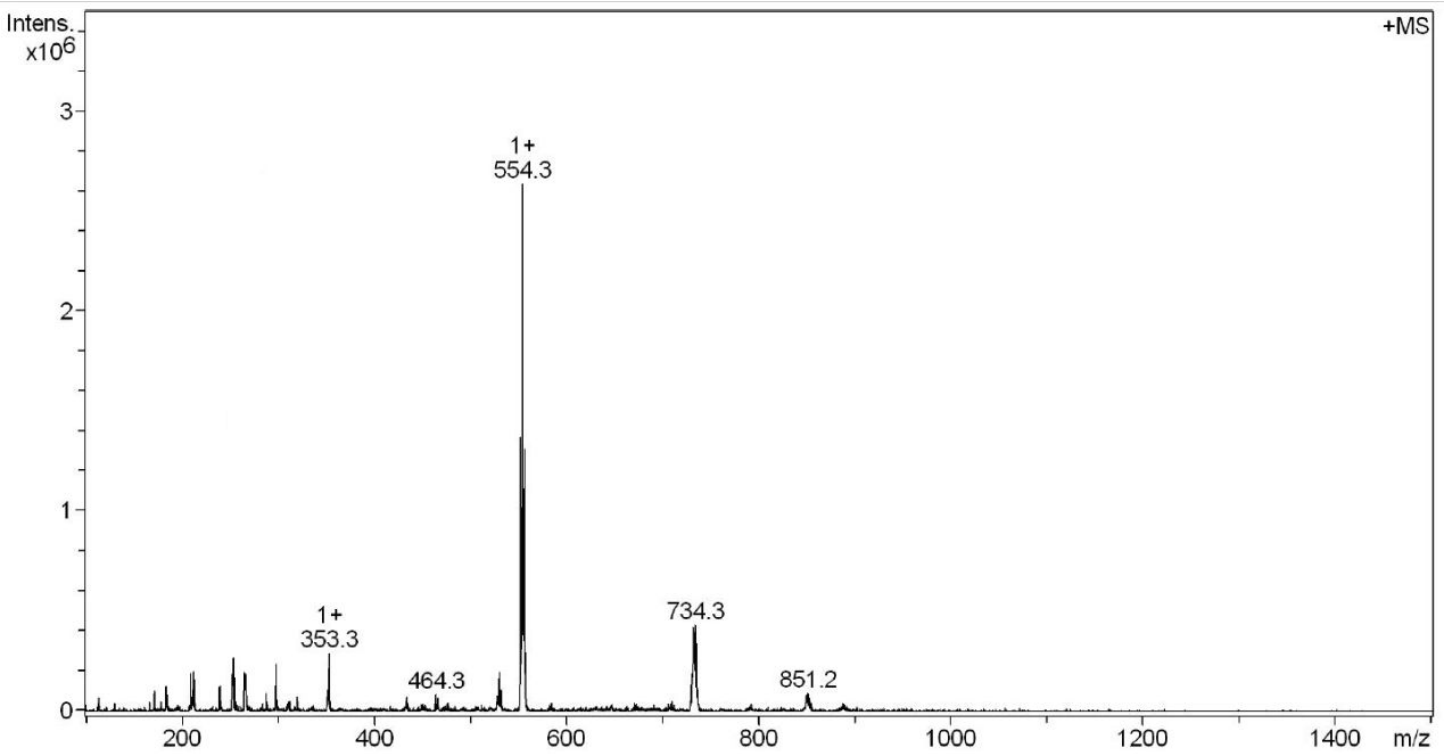

Figure S31. The mass spectra of $\mathbf{C o 4}\left(3.0 \times 10^{-5} \mathrm{M}\right)$ in Tris- $\mathrm{HCl}$ buffer solution (containing 5\% DMSO) for $0 \mathrm{~h}$.

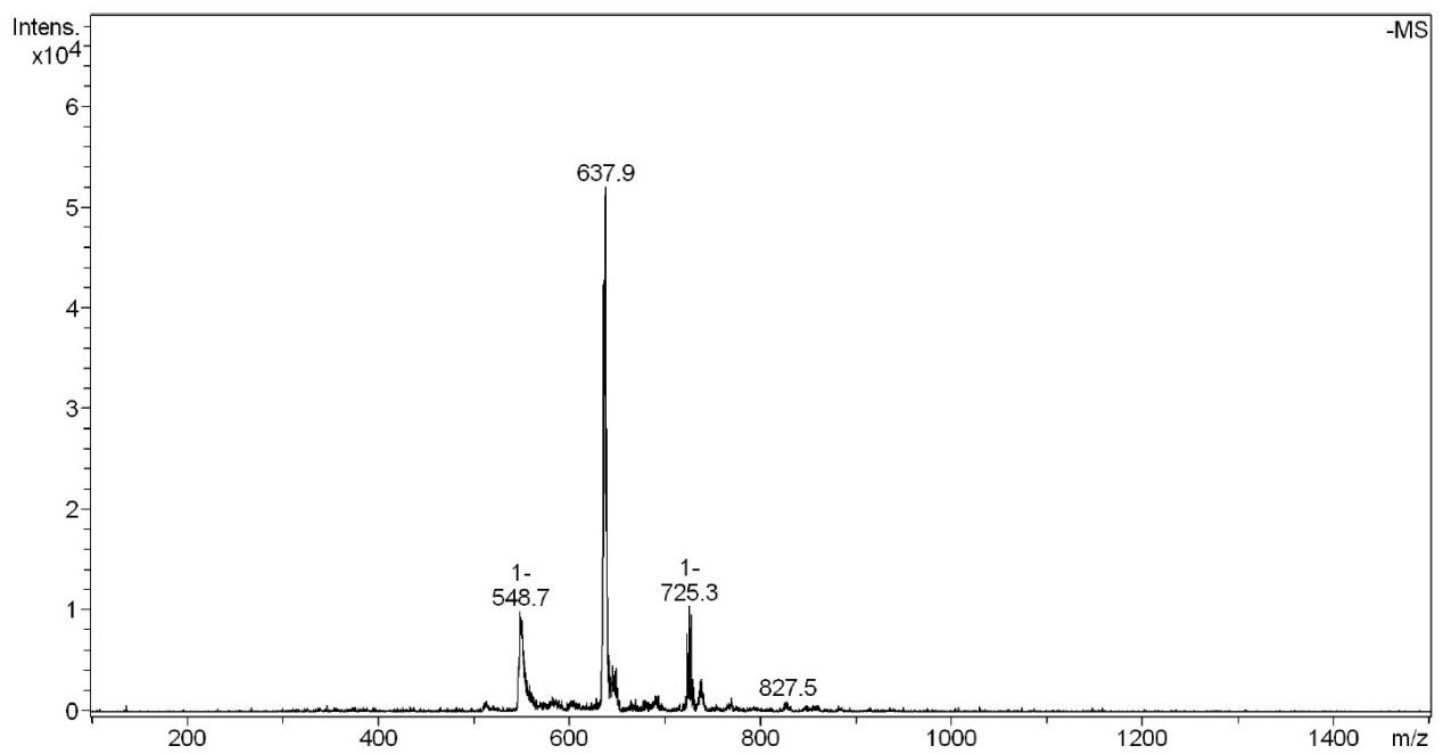

Figure S32. The mass spectra of $\mathbf{C o 5}\left(3.0 \times 10^{-5} \mathrm{M}\right)$ in Tris- $\mathrm{HCl}$ buffer solution (containing 5\% DMSO) for $0 \mathrm{~h}$. 


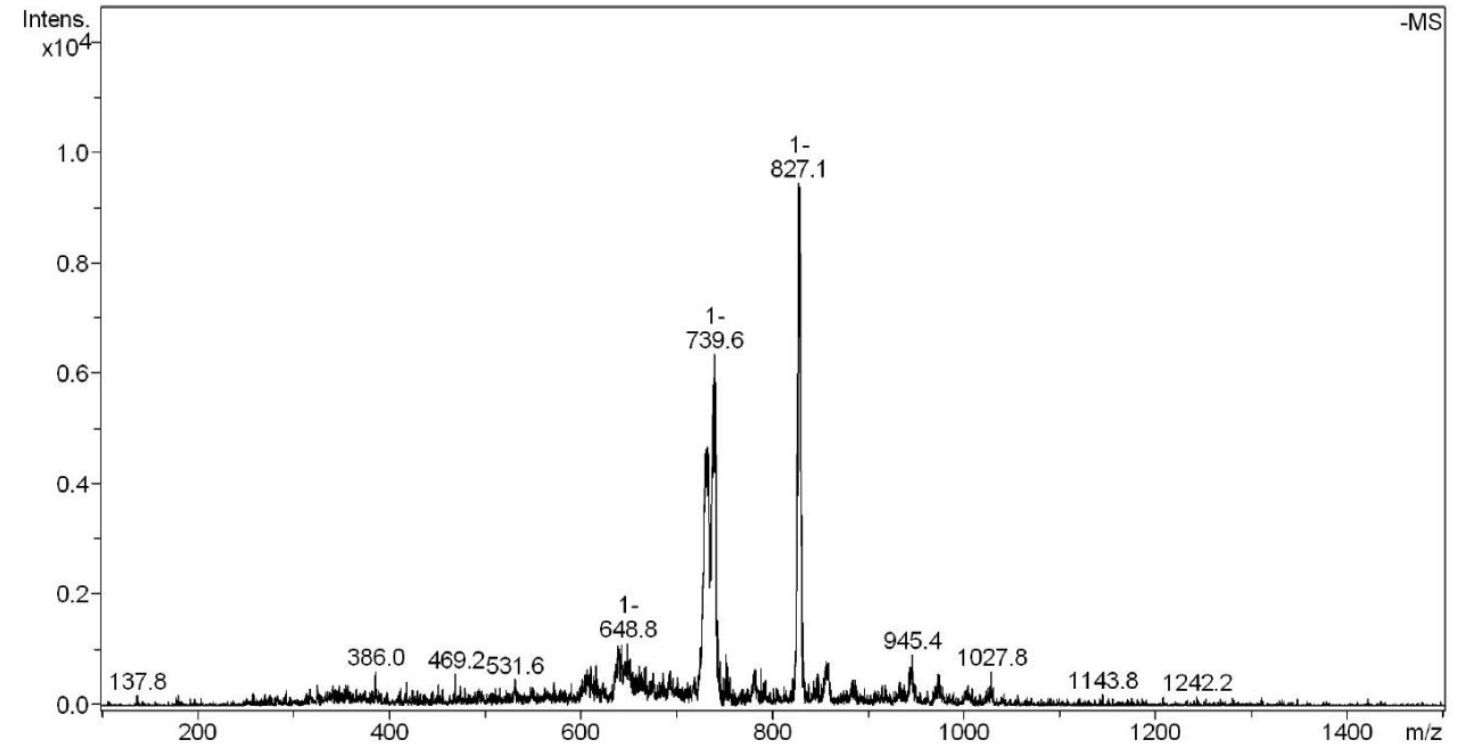

Figure S33. The mass spectra of $\mathbf{C o 6}\left(3.0 \times 10^{-5} \mathrm{M}\right)$ in Tris- $\mathrm{HCl}$ buffer solution (containing 5\% DMSO) for $0 \mathrm{~h}$.

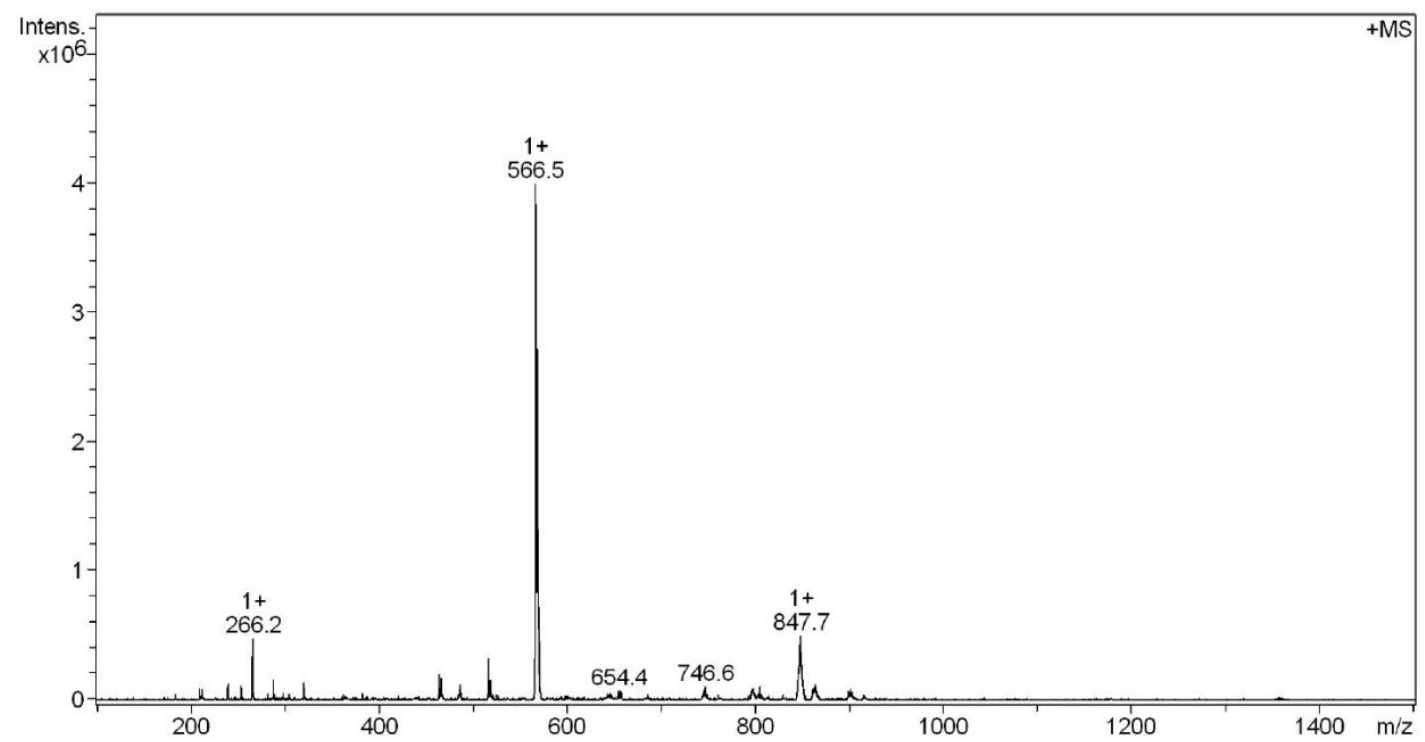

Figure S34. The mass spectra of $\mathbf{C o 7}\left(3.0 \times 10^{-5} \mathrm{M}\right)$ in Tris- $\mathrm{HCl}$ buffer solution (containing 5\% DMSO) for $0 \mathrm{~h}$. 


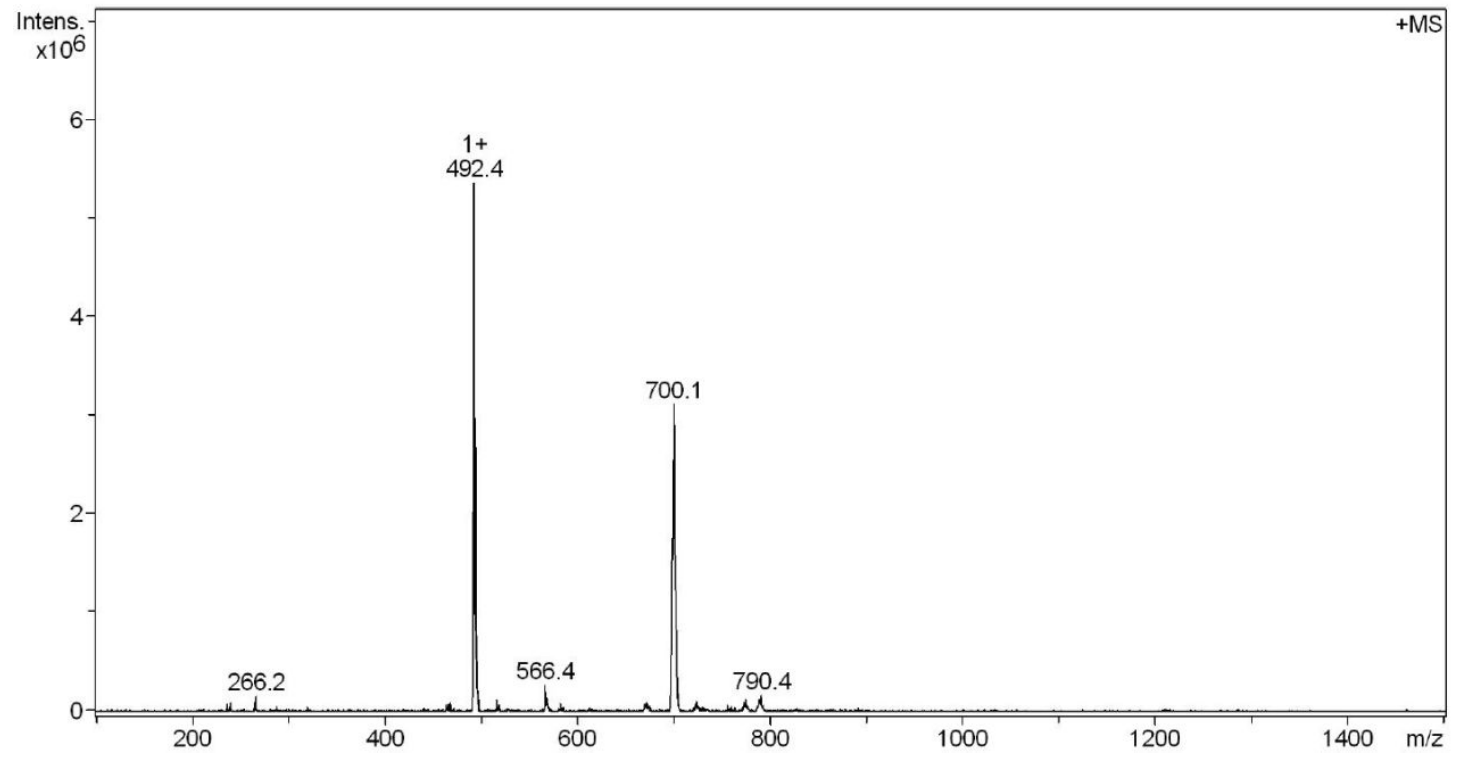

Figure S35. The mass spectra of $\mathbf{C o 8}\left(3.0 \times 10^{-5} \mathrm{M}\right)$ in Tris- $\mathrm{HCl}$ buffer solution (containing 5\% DMSO) for $0 \mathrm{~h}$.

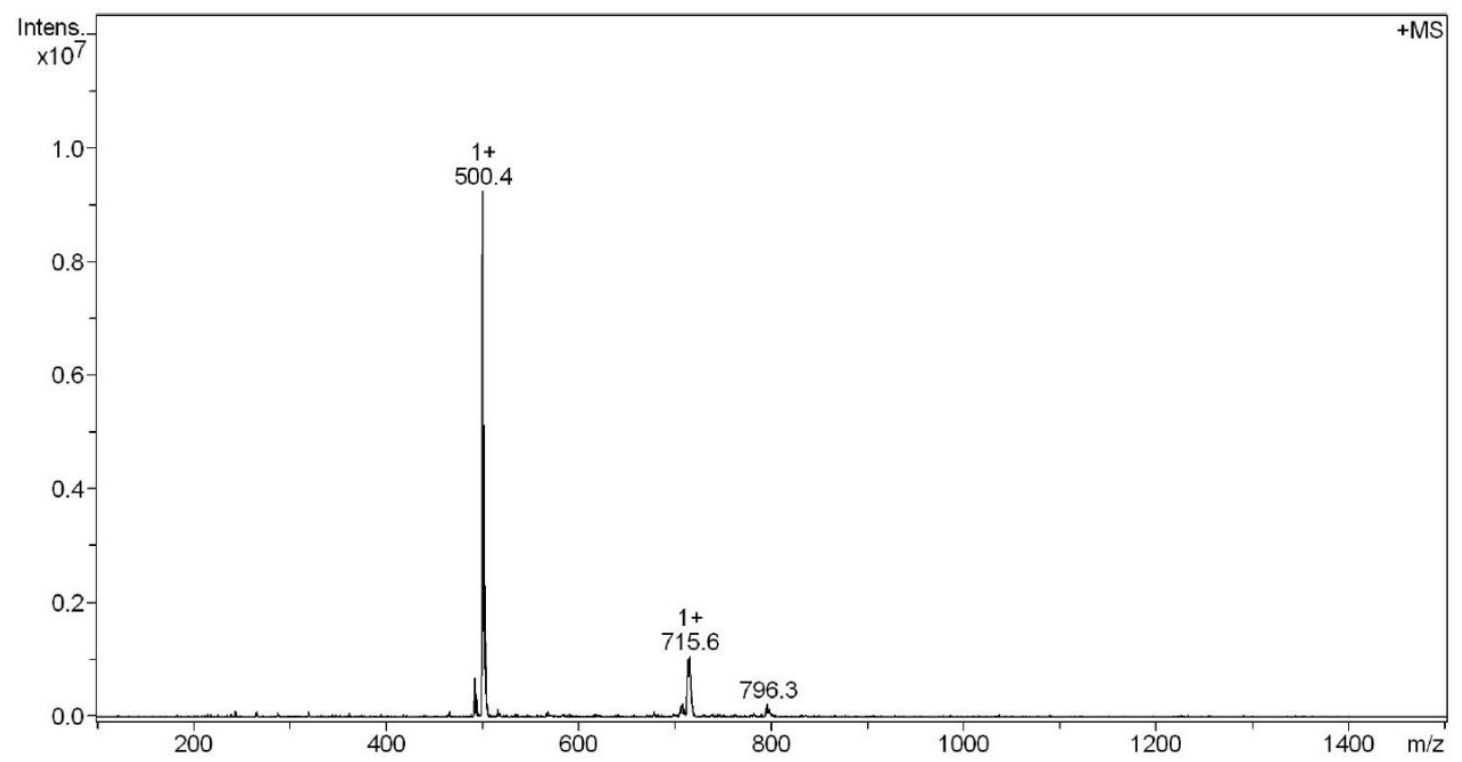

Figure S36. The mass spectra of $\mathbf{C o 9}\left(3.0 \times 10^{-5} \mathrm{M}\right)$ in Tris- $\mathrm{HCl}$ buffer solution (containing 5\% DMSO) for $0 \mathrm{~h}$. 


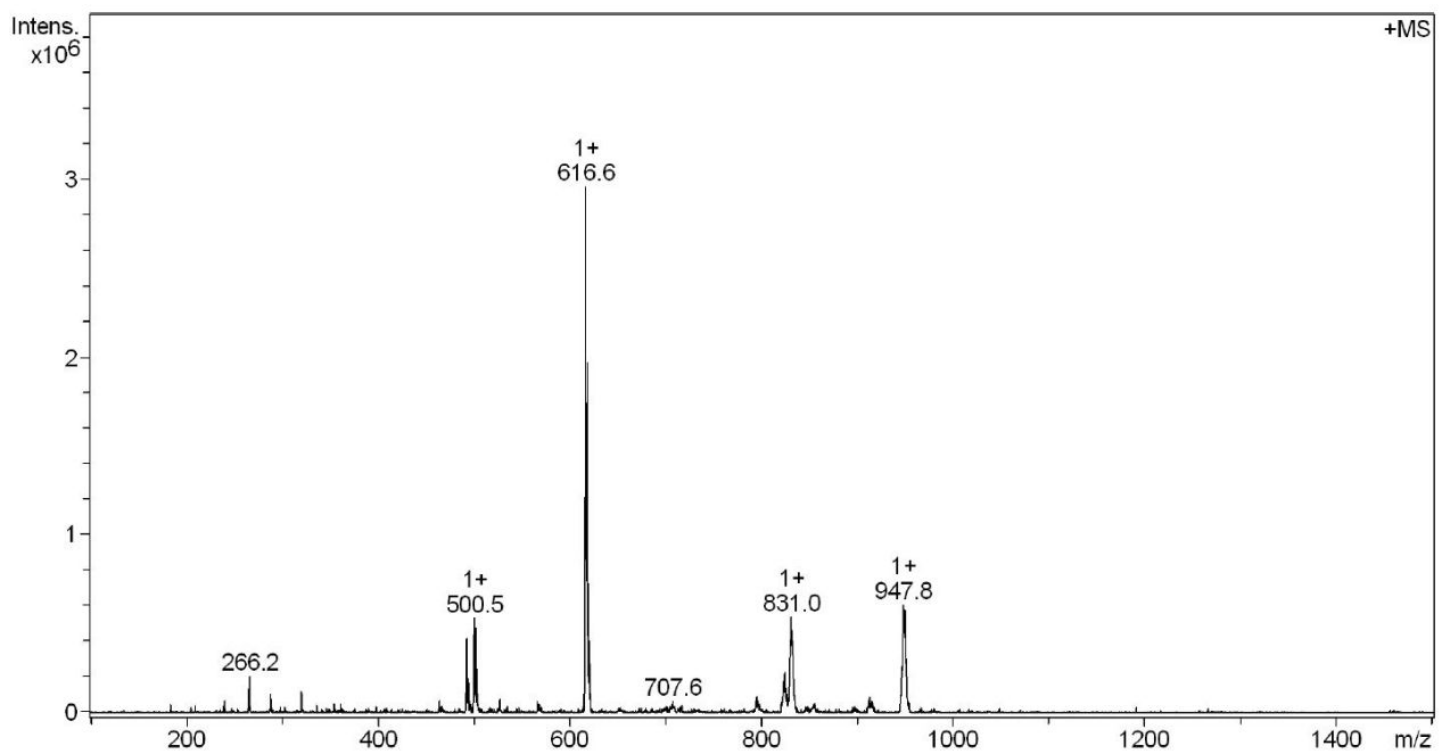

Figure S37. The mass spectra of $\mathbf{C o 1 0}\left(3.0 \times 10^{-5} \mathrm{M}\right)$ in Tris- $\mathrm{HCl}$ buffer solution (containing 5\% DMSO) for $0 \mathrm{~h}$.

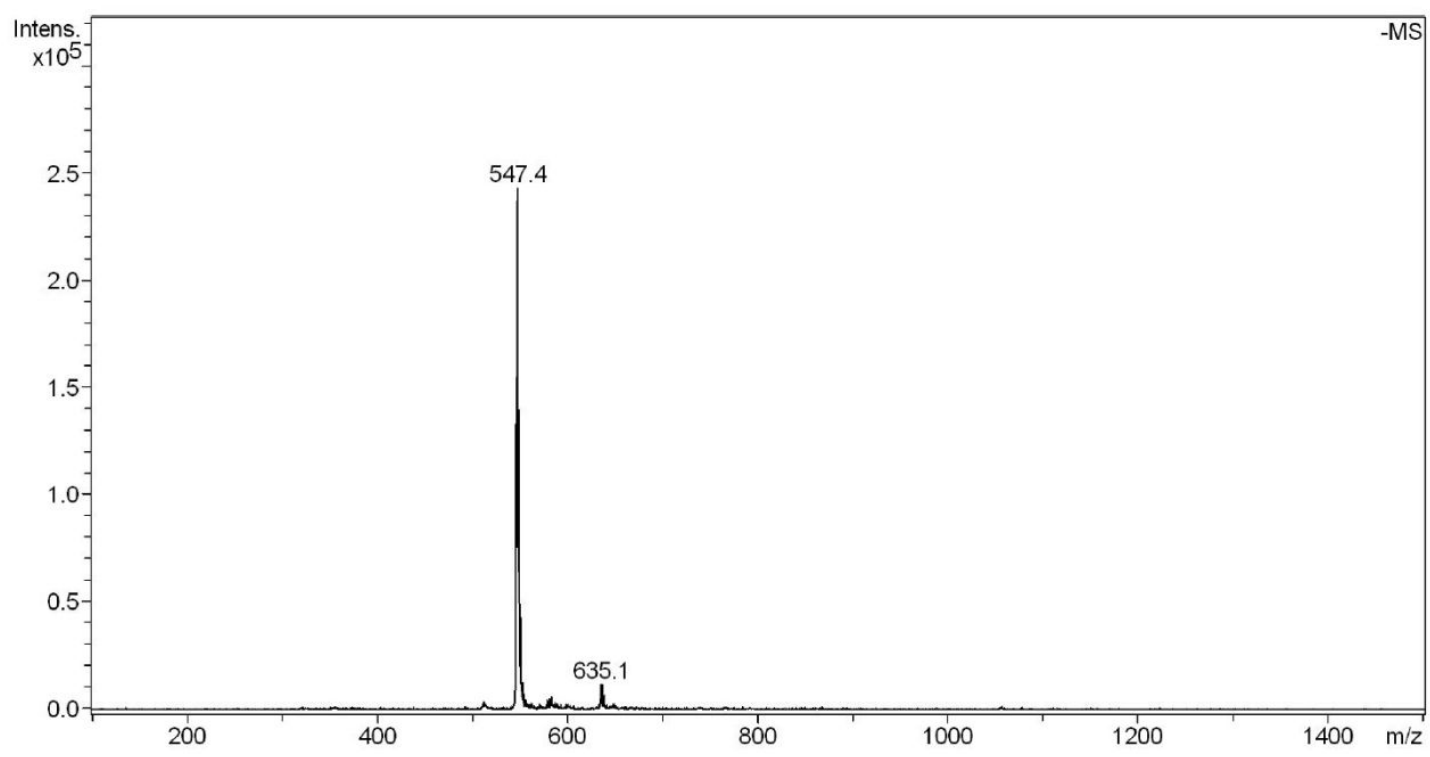

Figure S38. The mass spectra of Co11 $\left(3.0 \times 10^{-5} \mathrm{M}\right)$ in Tris- $\mathrm{HCl}$ buffer solution (containing 5\% DMSO) for $0 \mathrm{~h}$. 


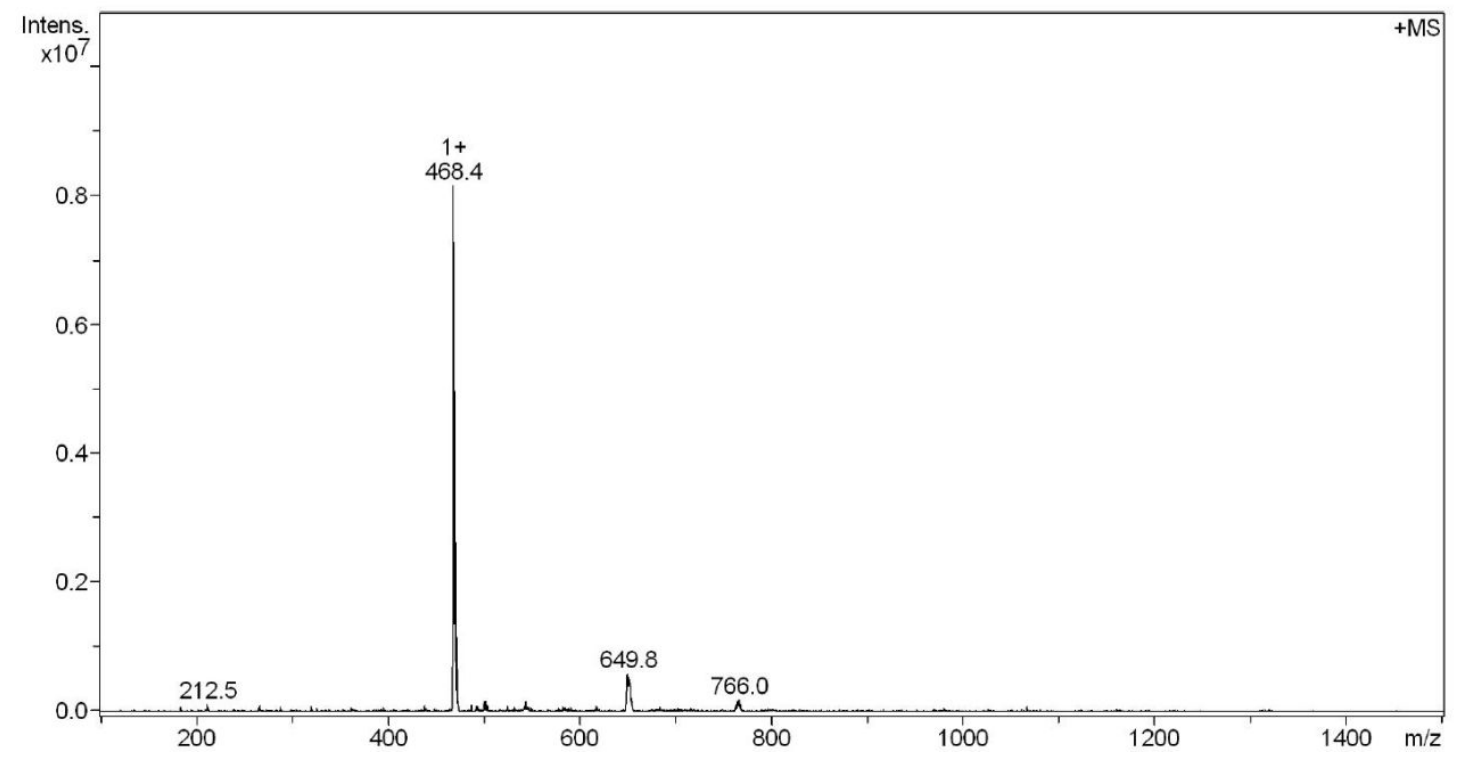

Figure S39. The mass spectra of $\mathbf{C o 1 2}\left(3.0 \times 10^{-5} \mathrm{M}\right)$ in Tris- $\mathrm{HCl}$ buffer solution (containing 5\% DMSO) for $0 \mathrm{~h}$.

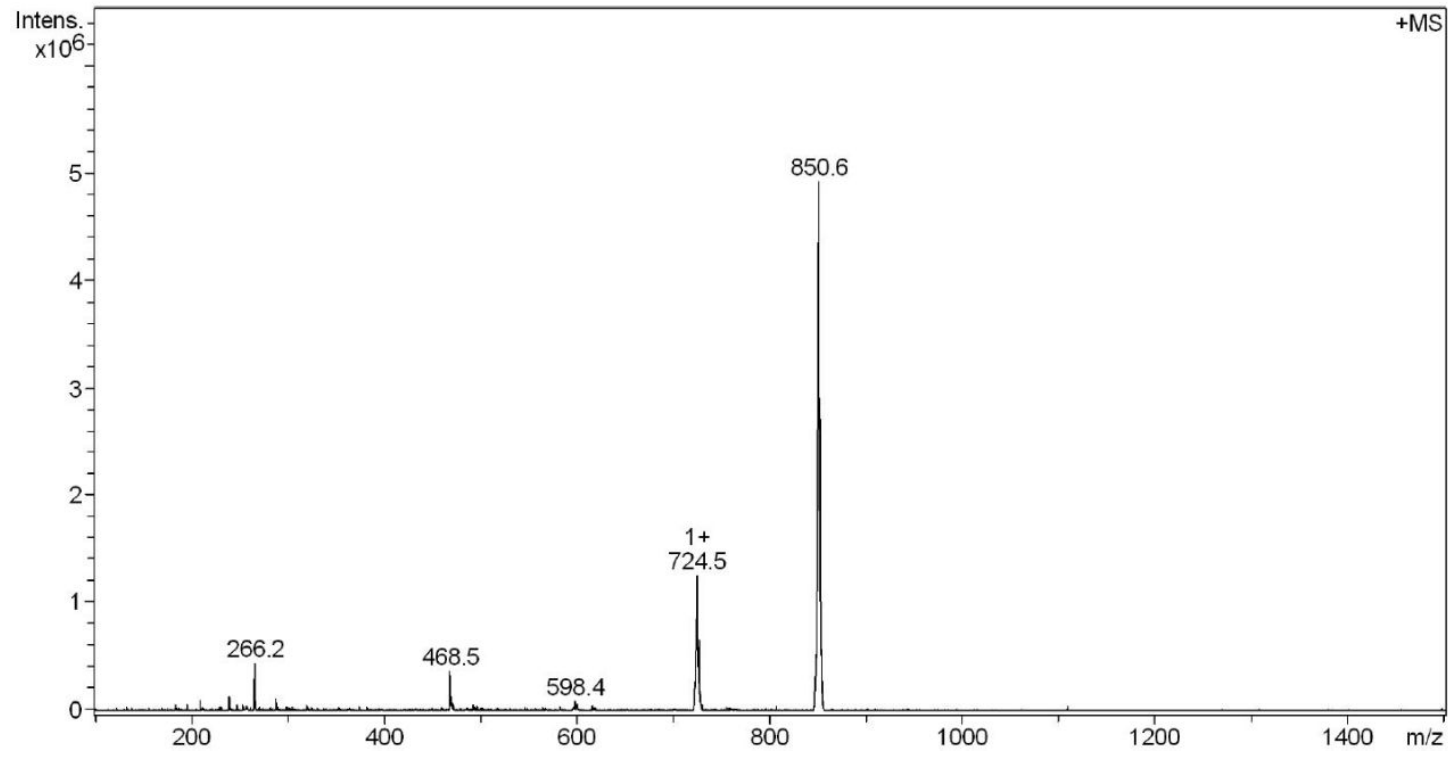

Figure S40. The mass spectra of $\mathbf{C o 1 3}\left(3.0 \times 10^{-5} \mathrm{M}\right)$ in Tris- $\mathrm{HCl}$ buffer solution (containing 5\% DMSO) for $0 \mathrm{~h}$. 


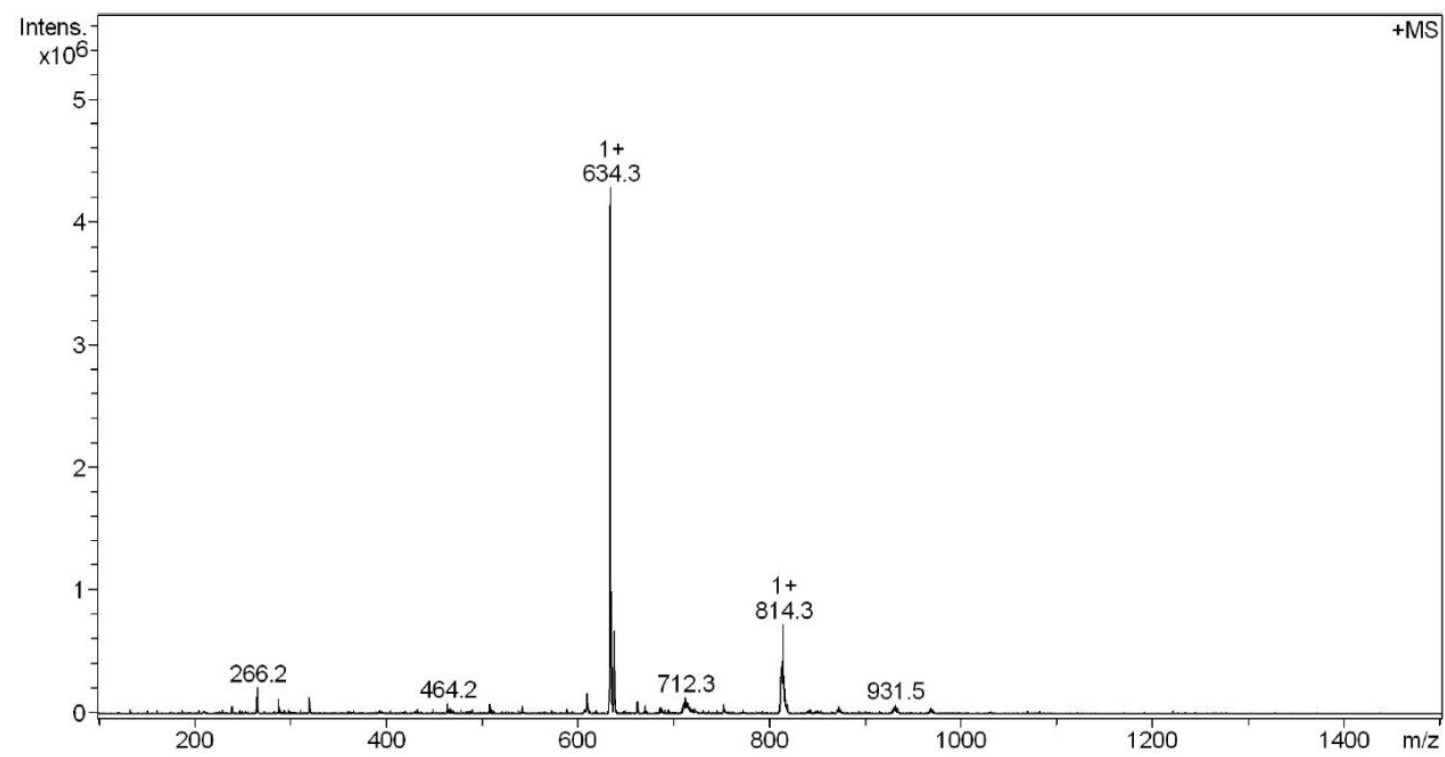

Figure S41. The mass spectra of Co14 $\left(3.0 \times 10^{-5} \mathrm{M}\right)$ in Tris- $\mathrm{HCl}$ buffer solution (containing 5\% DMSO) for $0 \mathrm{~h}$.

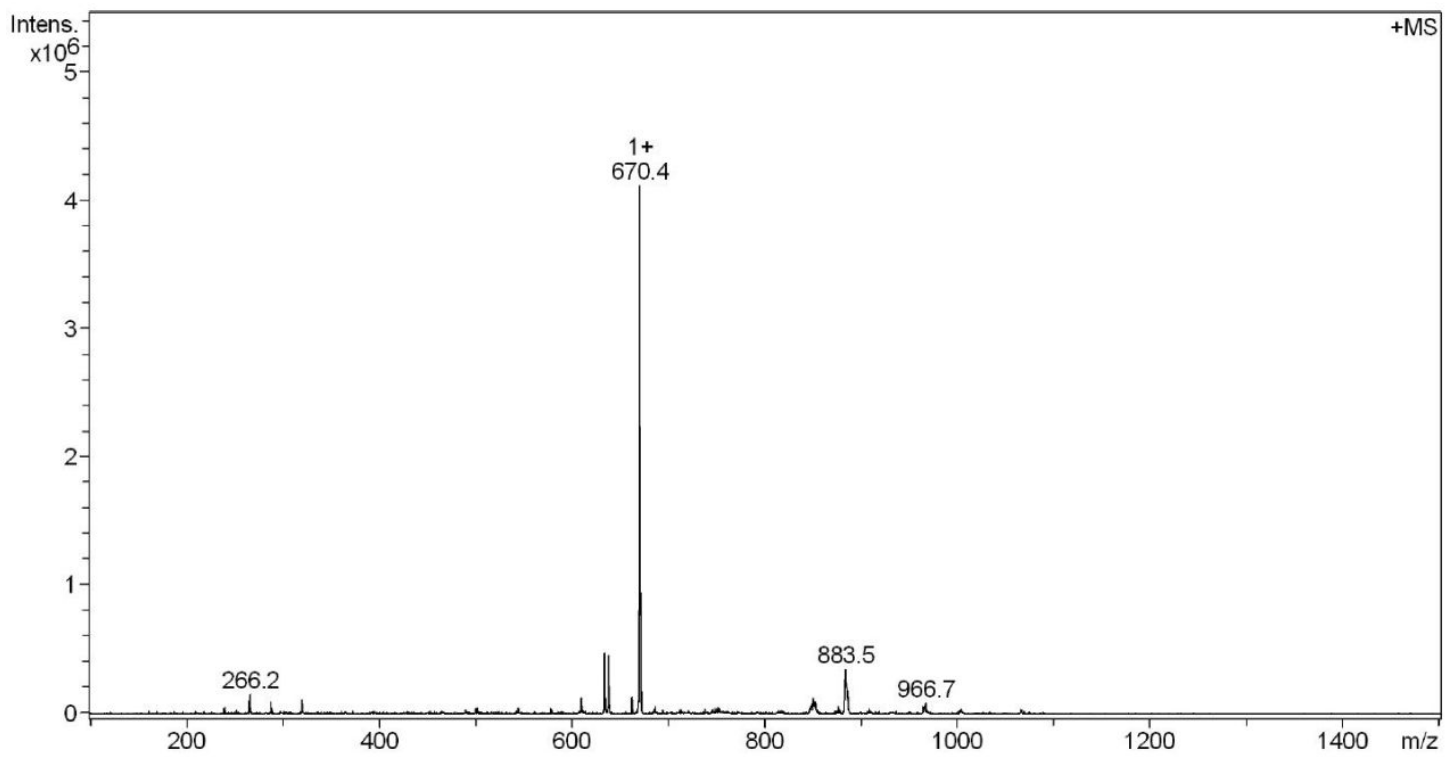

Figure S42. The mass spectra of $\mathbf{C o 1 5}\left(3.0 \times 10^{-5} \mathrm{M}\right)$ in Tris- $\mathrm{HCl}$ buffer solution (containing 5\% DMSO) for $0 \mathrm{~h}$. 


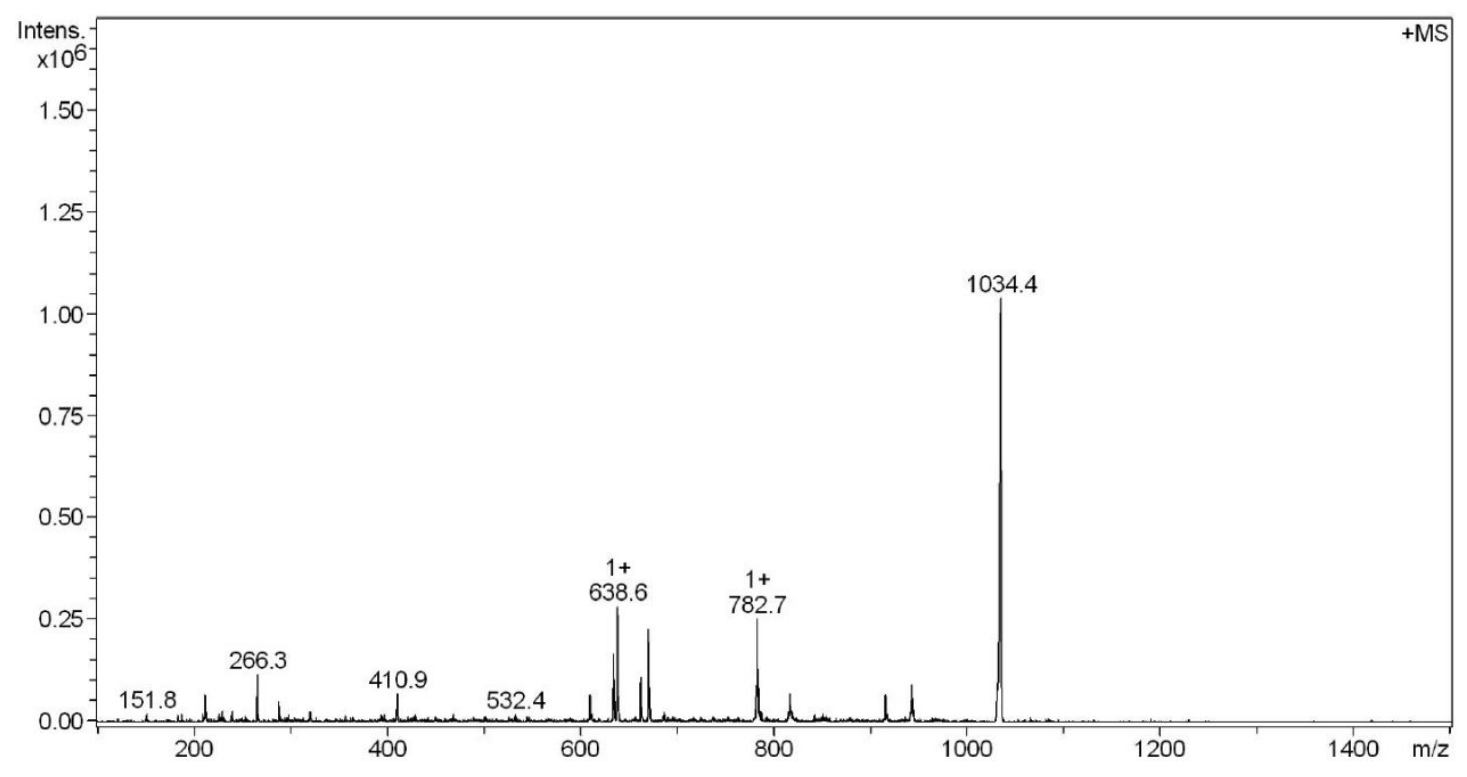

Figure S43. The mass spectra of Co16 $\left(3.0 \times 10^{-5} \mathrm{M}\right)$ in Tris- $\mathrm{HCl}$ buffer solution (containing 5\% DMSO) for $0 \mathrm{~h}$.

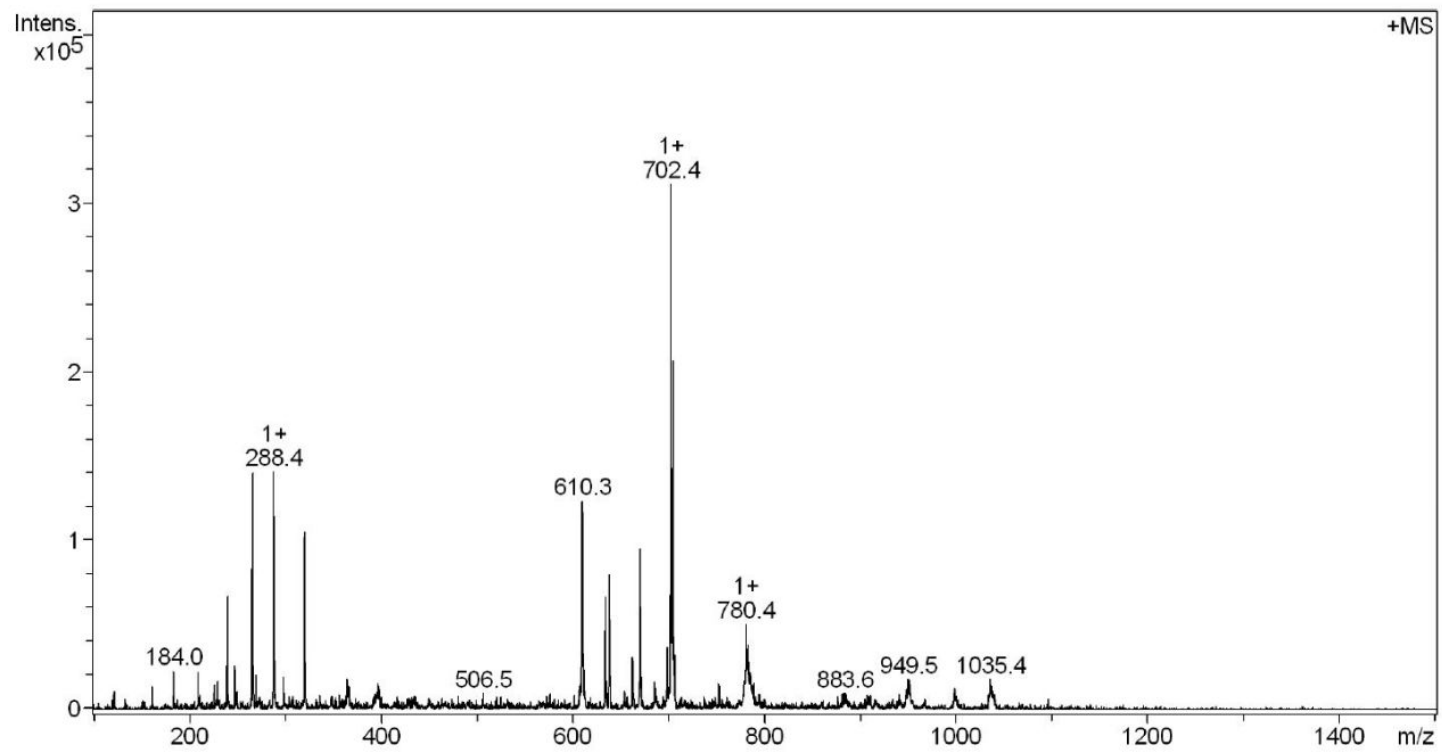

Figure S44. The mass spectra of $\mathrm{Co17}\left(3.0 \times 10^{-5} \mathrm{M}\right)$ in Tris- $\mathrm{HCl}$ buffer solution (containing 5\% DMSO) for $0 \mathrm{~h}$. 


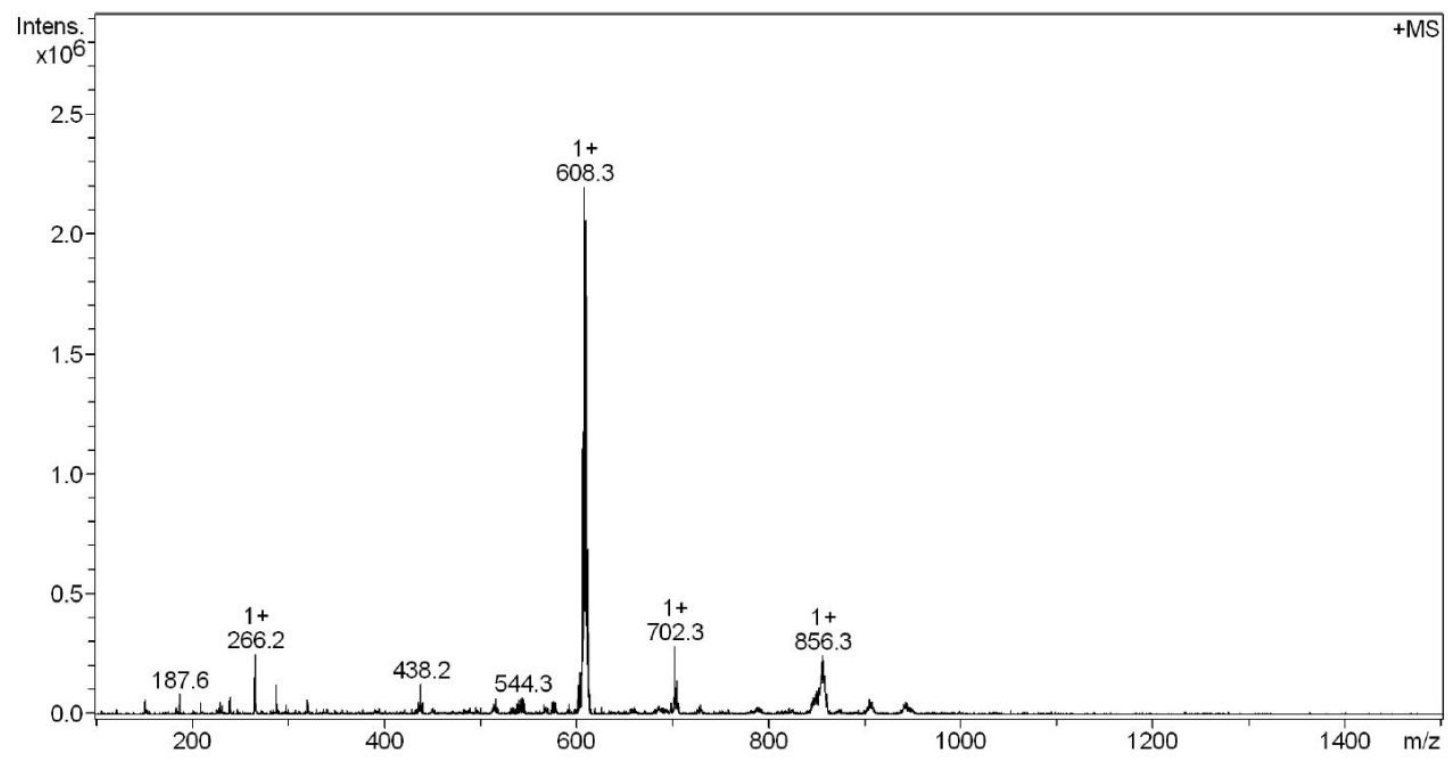

Figure S45. The mass spectra of $\mathbf{C o 1 8}\left(3.0 \times 10^{-5} \mathrm{M}\right)$ in Tris- $\mathrm{HCl}$ buffer solution (containing 5\% DMSO) for $0 \mathrm{~h}$.
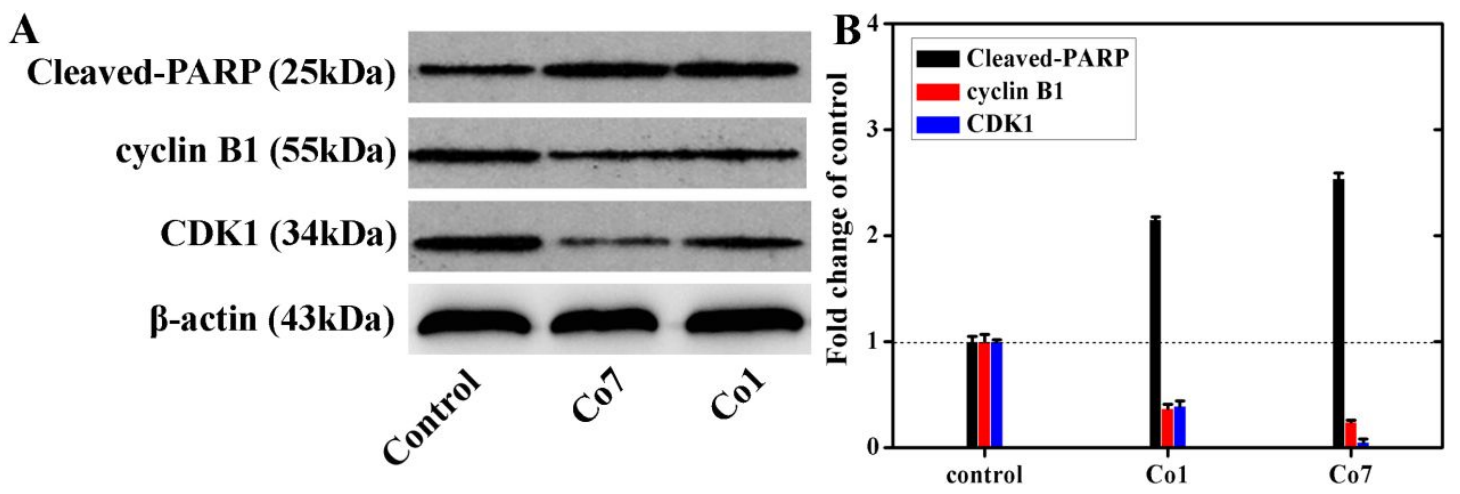

Fig. S46. Co7 $(0.80 \mathrm{nM})$ and Co1 $(4.53 \mu \mathrm{M})$ inhibited the expression of cyclin B1, cleaved-PARP and CDK1 (A and B) in HeLa cells at $24 \mathrm{~h}$. 


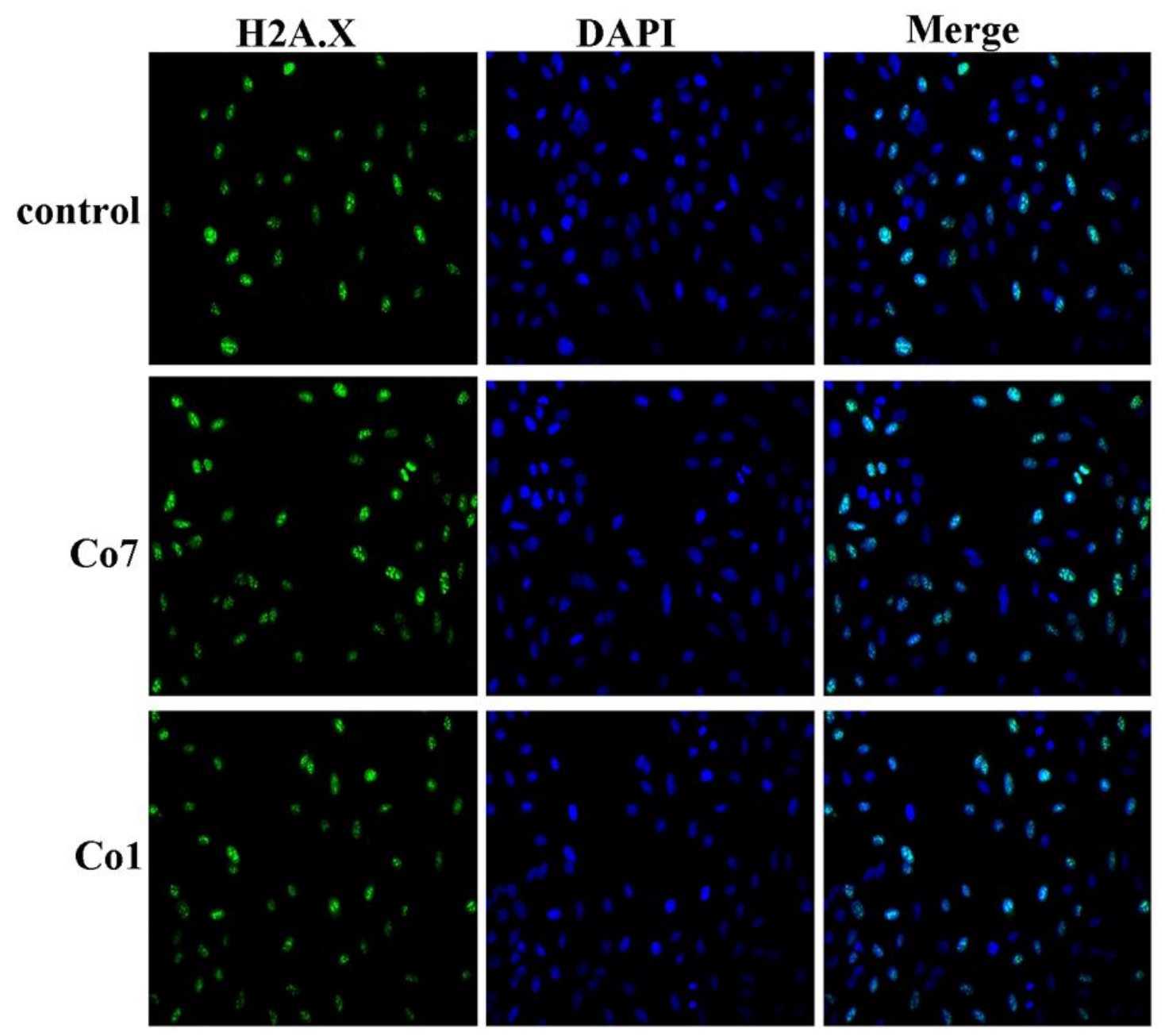

Fig. S47. Co7 $(0.80 \mathrm{nM})$ and $\mathrm{Co1}(4.53 \mu \mathrm{M})$ up-regulated the expression of H2A.X in HeLa cells for $24 \mathrm{~h}$.
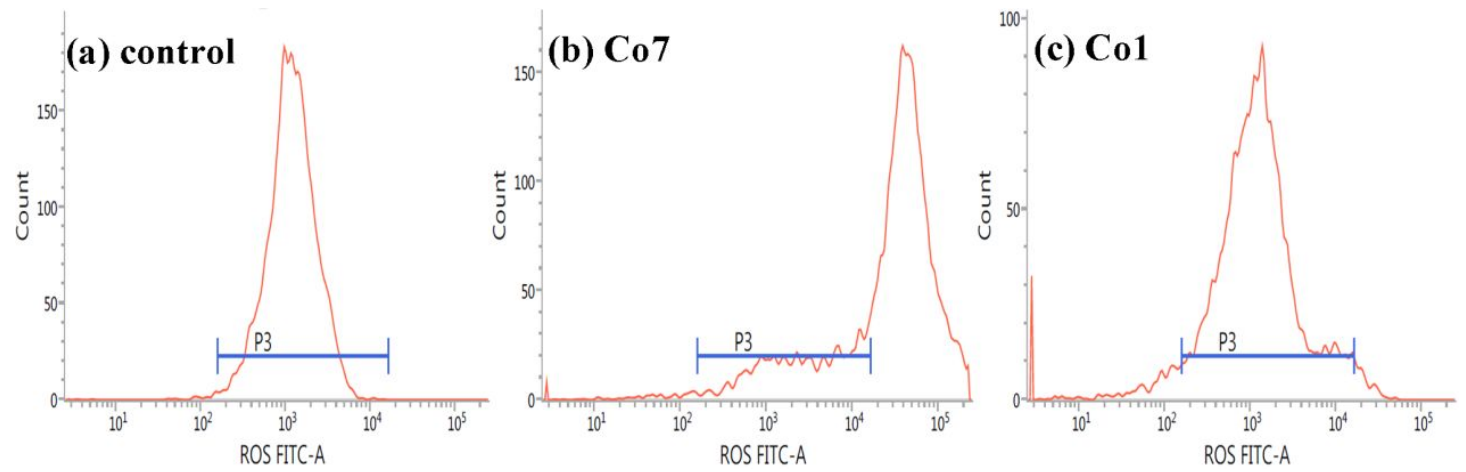

Fig. S48. ROS generation assay by flow cytometric analysis of HeLa cells after treated with $\mathbf{C o} 7(0.80 \mathrm{nM})$ and $\mathbf{C o 1}(4.53 \mu \mathrm{M})$, respectively. 

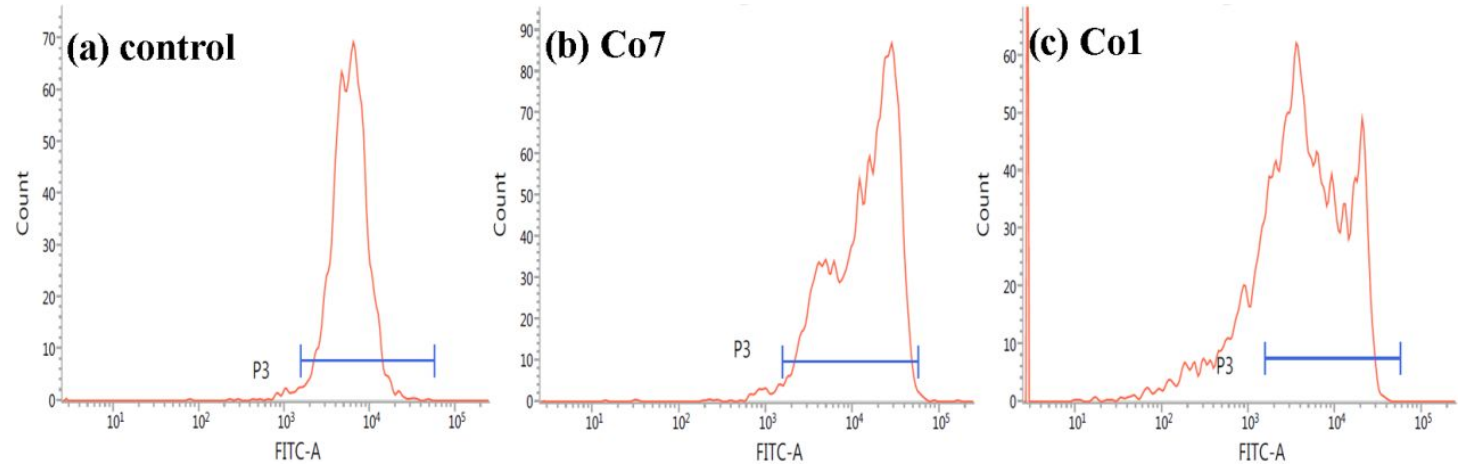

Fig. S49. Caspase-3 activation of HeLa cells treatment with Co7 (0.80 nM) and Co1 $(5.23 \mu \mathrm{M})$ for $24 \mathrm{~h}$, respectively.
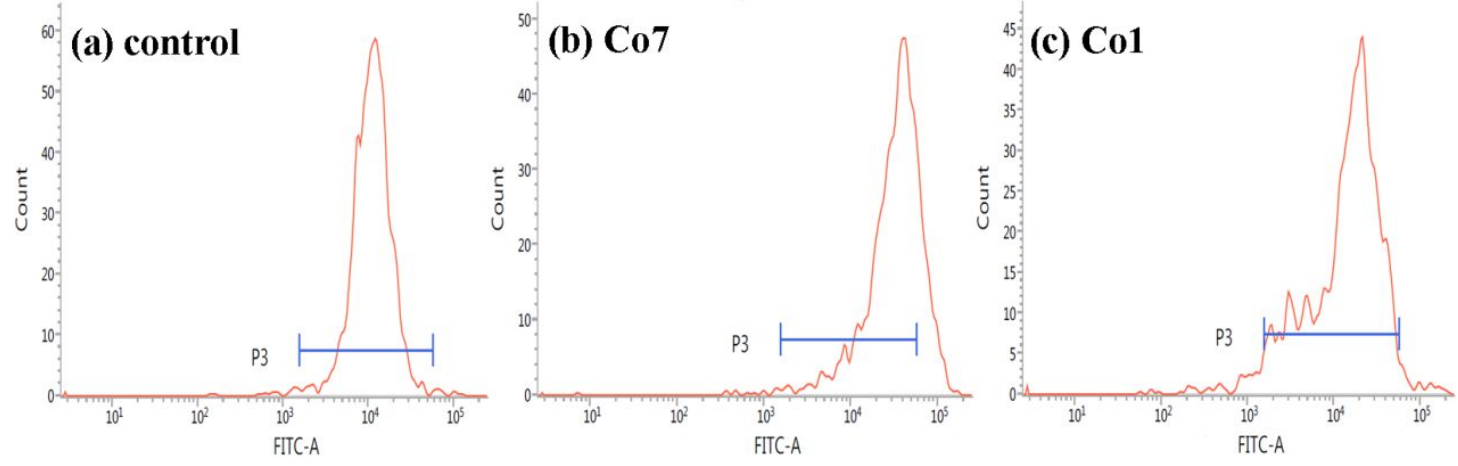

Fig. S50. Caspase-9 activation of HeLa cells treatment with Co7 $(0.80 \mathrm{nM})$ and

Co1 $(4.53 \mu \mathrm{M})$ for $24 \mathrm{~h}$, respectively.
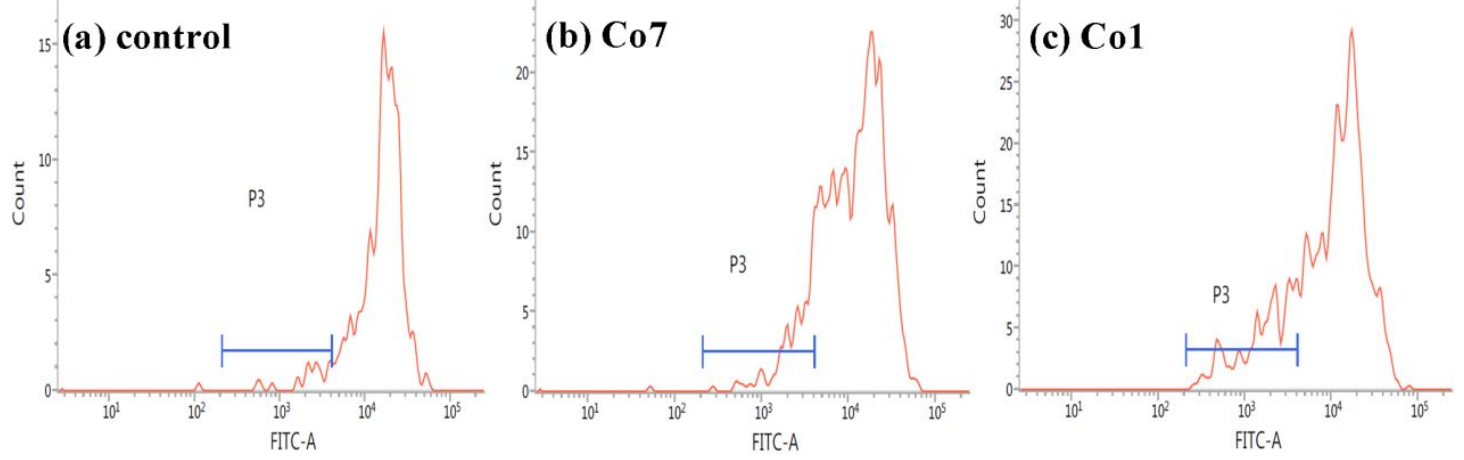

Fig. S51. The level of $\Delta \Psi \mathrm{m}$ in HeLa cells treatment of $\mathbf{C o 7}(0.80 \mathrm{nM})$ and $\mathbf{C o 1}$ $(4.53 \mu \mathrm{M})$ for $24 \mathrm{~h}$, respectively. 
Table S57. The tumor volume in treated and non-treated mice from the date of surgery to the study end point in the Hela xenograft model.

\begin{tabular}{cccc}
\hline Group & \multicolumn{2}{c}{ Tumor Volume $\left(\mathbf{m m}^{\mathbf{3}}\right)$} & T/C \\
& (start) & (end) & (\%) \\
\hline Control & $82.4 \pm 27.0$ & $1127.8 \pm 180.7$ & - \\
Co7 $(2.0 \mathrm{mg} / \mathrm{kg})$ & $82.4 \pm 27.0$ & $613.1 \pm 98.5$ & $53.4^{\mathrm{a}}$ \\
\hline${ }^{\mathrm{a}}$ mean $p<0.05, \quad p$ vs vehicle control & &
\end{tabular}

Table S58. Average body weight in treated and non-treated mice from the date of surgery to the study end point in the HeLa xenogfart model.

\begin{tabular}{clcc}
\hline \multirow{2}{*}{ Group } & \multicolumn{2}{c}{ Body Weight (g) } & RBW (\%) \\
& (start) & (end) & (end) \\
\hline Control & $18.7 \pm 1.2$ & $20.7 \pm 1.4$ & 110.7 \\
Co7 $(2.0 \mathrm{mg} / \mathrm{kg})$ & $18.8 \pm 1.3$ & $20.5 \pm 1.3$ & 109.0 \\
\hline
\end{tabular}

Table S59. In Vivo Anticancer Activity of Co7 toward Hela Tumor Xenograft.

\begin{tabular}{ccc}
\hline Group & $\begin{array}{c}\text { average tumor } \\
\text { weight(mean } \pm \text { SD g) }\end{array}$ & $\begin{array}{c}\text { inhibition of tumor } \\
\text { growth(\%) }\end{array}$ \\
\hline Control & $1.562 \pm 0.148$ & - \\
Co7 $(2.0 \mathrm{mg} / \mathrm{kg})$ & $0.880 \pm 0.102$ & 43.7 \\
\hline
\end{tabular}

\footnotetext{
${ }^{\mathrm{a}}$ mean $p<0.05, \quad p v s$ control.
} 


\section{Experimental methods}

The synthesis of 1,10-phenanthroline (Phen), dipyridophenazine (DPPZ), 2,2'-dipyridyl (dpy), and dipyridoquinoxaline (DPQ) were carried out according to Kellett et al. reported methods ${ }^{1}$.

\section{Synthesis of Co1-Co18}

The 18 red-brown novel Co(II) complexes Co1-Co18 were prepared by $\mathrm{CH}_{3} \mathrm{CN}-\mathrm{CH}_{3} \mathrm{OH}(3.5 \mathrm{~mL} / 1.5 \mathrm{~mL})$ reflux of $2.0 \mathrm{mmol} \mathrm{H}-\mathrm{QL} 1$ (or $\mathrm{H}-\mathrm{QL} 2$, H-QL3, H-QL4, H-QL5) and $1.0 \mathrm{mmol}$ py, Phen, DPQ, DPPZ, MDP, ODP, PPT, ClPT, dpy and mpy with $1.0 \mathrm{mmol} \mathrm{CoCl}_{2} \cdot 6 \mathrm{H}_{2} \mathrm{O}(2: 1: 1)$ at $80{ }^{\circ} \mathrm{C}$ for $24 \mathrm{~h}$, respectively. And the resulting red-brown product suitable for structural characterization were isolated, washed with $\mathrm{CH}_{3} \mathrm{CH}_{2} \mathrm{OH}$ and ether, and air-dried, respectively.

Data for Co1. Yield: 80.2\%. Elemental analysis calcd (\%) for $\mathrm{C}_{30} \mathrm{H}_{20} \mathrm{Cl}_{4} \mathrm{CoN}_{4} \mathrm{O}_{2}$ : C 53.84, $\mathrm{H}$ 3.01, $\mathrm{N}$ 8.37; found: C 53.80, H 3.03, N 8.35. IR (KBr): 2922, 1596, 1544, 1453, 1432, 1356, 1248, 1152, 1110, 1019, 952, 870, 819, 772, 758, 736, 694, 651, 535, 445, $408 \mathrm{~cm}^{-1}$. ESI-MS m/z: 440.3 $[\mathrm{M}-(\mathrm{QL} 1)]^{+}$(Tris-HCl buffer solution containing 5\% DMSO as solvent).

Data for Co2. Yield: 75.0\%. Elemental analysis calcd (\%) for $\mathrm{C}_{30} \mathrm{H}_{20} \mathrm{Br}_{4} \mathrm{CoN}_{4} \mathrm{O}_{2}$ : C 42.54, $\mathrm{H}$ 2.38, $\mathrm{N}$ 6.61; found: C 42.52, H 2.41, N 6.63. IR (KBr): 3431, 2924, 2856, 1596, 1541, 1488, 1427, 1349, 1275, 1245, 1149 , 1108, 1017, 931, 872, 818, 757, 737, 663, 647, 527, 468, $439 \mathrm{~cm}^{-1}$. ESI-MS $\mathrm{m} / \mathrm{z}: 530.3[\mathrm{M}-(\mathrm{QL} 2)]^{+}$(Tris-HCl buffer solution containing 5\% DMSO as solvent).

Data for Co3. Yield: 85.9\%. Elemental analysis calcd (\%) for $\mathrm{C}_{32} \mathrm{H}_{20} \mathrm{Cl}_{4} \mathrm{CoN}_{4} \mathrm{O}_{2}$ : C 55.44, $\mathrm{H}$ 2.91, $\mathrm{N}$ 8.08; found: C 55.40, H 2.95, N 8.06. IR (KBr): 2924, 2856, 1544, 1495, 1452, 1427, 1356, 1277, 1252, 1149, 1110 , 1024, 953, 871, 846, 772, 727, 695, 653, 536, $445 \mathrm{~cm}^{-1}$. ESI-MS m/z: 725.8 $[\mathrm{M}+\mathrm{Cl}]^{-}($Tris- $\mathrm{HCl}$ buffer solution containing 5\% DMSO as solvent).

Data for Co4. Yield: 70.1\%. Elemental analysis calcd (\%) for $\mathrm{C}_{32} \mathrm{H}_{20} \mathrm{Br}_{4} \mathrm{CoN}_{4} \mathrm{O}_{2}$ : C 44.12, $\mathrm{H} 2.31, \mathrm{~N}$ 6.43; found: C 44.10, H 2.35, N 6.40. IR (KBr): 3408, 3061, 2923, 1541, 1511, 1425, 1350, 1274, 1244, 1144, 1107, 
1019, 931, 870, 844, 821, 728, 663, 644, 528, 467, $438 \mathrm{~cm}^{-1}$. ESI-MS m/z:

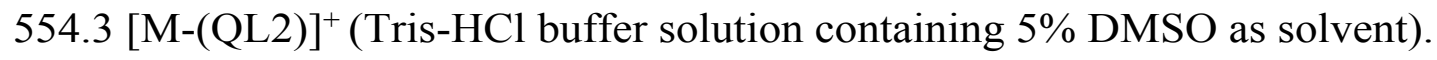

Data for Co5. Yield: 90.5\%. Elemental analysis calcd (\%) for $\mathrm{C}_{34} \mathrm{H}_{20} \mathrm{Cl}_{4} \mathrm{CoN}_{6} \mathrm{O}_{2}$ : C 54.79, H 2.70, N 11.28; found: C 54.77, H 2.73, N 11.26. IR (KBr): 3371, 1546, 1427, 1359, 1252, 1113, 1082, 1030, 955, 867, 817, 772, 739, 695, 653, 537, $444 \mathrm{~cm}^{-1}$. ESI-MS m/z: $637.9\left[\mathrm{M}-(\mathrm{QL} 1)+2 \mathrm{Cl}+3 \mathrm{H}_{2} \mathrm{O}\right]^{-}$ (Tris- $\mathrm{HCl}$ buffer solution containing 5\% DMSO as solvent).

Data for Co6. Yield: 85.5\%. Elemental analysis calcd (\%) for $\mathrm{C}_{34} \mathrm{H}_{20} \mathrm{Br}_{4} \mathrm{CoN}_{6} \mathrm{O}_{2}$ : C 44.24, $\mathrm{H}$ 2.18, N 9.10; found: C 44.21, H 2.22, N 9.09. IR (KBr): 3071, 2921, 1580, 1541, 1490, 1425, 1352, 1272, 1244, 1211, 1110 , 1081, 1029, 932, 875, 815, 737, 664, 646, 530, $440 \mathrm{~cm}^{-1}$. ESI-MS m/z: 827.1 $\left[\mathrm{M}-(\mathrm{QL} 2)+2 \mathrm{Cl}+2 \mathrm{DMSO}^{-}\right.$(Tris-HCl buffer solution containing 5\% DMSO as solvent).

Data for Co7. Yield: 89.2\%. Elemental analysis calcd (\%) for $\mathrm{C}_{38} \mathrm{H}_{22} \mathrm{Cl}_{4} \mathrm{CoN}_{4} \mathrm{O}_{2}$ : C 59.48, H 2.89, N 7.30; found: C 59.44, H 2.87, N 7.27. IR (KBr): 3398, 3062, 2923, 1547, 1492, 1423, 1357, 1253, 1075, 1038, 955, 819, 771, 738, 695, 538, $455 \mathrm{~cm}^{-1}$. ESI-MS m/z: 566.5 [M-(QL1) $]^{+}$(Tris-HCl buffer solution containing 5\% DMSO as solvent).

Data for Co8. Yield: 70.3\%. Elemental analysis calcd (\%) for $\mathrm{C}_{34} \mathrm{H}_{24} \mathrm{Cl}_{4} \mathrm{CoN}_{4} \mathrm{O}_{2}$ : C 56.61, H 3.35, N 7.77; found: C 56.59, H 3.37, N 7.74. IR (KBr): 3776, 3659, 3461, 3367, 1604, 1547, 1425, 1355, 1256, 1109, 1029, 946, 865, 813, 769, 737, 692, 652, 537, $452 \mathrm{~cm}^{-1}$. ESI-MS m/z: 492.4 $[\mathrm{M}-(\mathrm{QL} 1)]^{+}$(Tris-HCl buffer solution containing 5\% DMSO as solvent).

Data for Co9. Yield: 80.4\%. Elemental analysis calcd (\%) for $\mathrm{C}_{32} \mathrm{H}_{24} \mathrm{Cl}_{4} \mathrm{CoN}_{4} \mathrm{O}_{4}$ : C 52.70, H 3.32, N 7.68; found: C 52.67, H 3.34, N 7.69. IR (KBr): 3777, 3437, 1610, 1556, 1425, 1358, 1252, 1033, 951, 827, 741, 693, $451 \mathrm{~cm}^{-1}$. ESI-MS m/z: $500.4[\mathrm{M}-(\mathrm{QL} 1)]^{+}$(Tris-HCl buffer solution containing $5 \%$ DMSO as solvent).

Data for Co10. Yield: 68.5\%. Elemental analysis calcd (\%) for $\mathrm{C}_{44} \mathrm{H}_{28} \mathrm{Cl}_{4} \mathrm{CoN}_{4} \mathrm{O}_{2}$ : C 62.51, H 3.34, N 6.63; found: C 62.50, H 3.37, N 6.61. IR (KBr): 3435, 1546, 1496, 1431, 1360, 1259, 1109, 1024, 953, 856, 769, 702, $453 \mathrm{~cm}^{-1}$. ESI-MS m/z: $616.6[\mathrm{M}-(\mathrm{QL} 1)]^{+}$(Tris-HCl buffer solution containing 5\% DMSO as solvent). 
Data for Co11. Yield: 90.8\%. Elemental analysis calcd (\%) for $\mathrm{C}_{32} \mathrm{H}_{18} \mathrm{Cl}_{6} \mathrm{CoN}_{4} \mathrm{O}_{2}$ : C 50.43, H 2.38, N 7.35; found: C 50.40, H 2.40, N 7.33. IR (KBr): 3437, 1553, 1500, 1435, 1357, 1252, 1110, 952, 835, 776, 739, 649 $\mathrm{cm}^{-1}$. ESI-MS m/z: 547.4 [M-(QL1)-2H+ $\left.\mathrm{H}_{2} \mathrm{O}\right]^{-}$(Tris- $\mathrm{HCl}$ buffer solution containing 5\% DMSO as solvent).

Data for Co12. Yield: 63.9\%. Elemental analysis calcd (\%) for $\mathrm{C}_{30} \mathrm{H}_{20} \mathrm{Cl}_{2} \mathrm{CoI}_{2} \mathrm{~N}_{4} \mathrm{O}_{2}$ : C 42.28, H 2.37, N 6.57; found: C 42.24, H 2.39, N 6.55. IR (KBr): 3777, 3446, 1615, 1548, 1439, 1357, 1248, 1022, 951, 825, 739 $\mathrm{cm}^{-1}$. ESI-MS m/z: $468.4[\mathrm{M}-(\mathrm{QL} 3)]^{+}$(Tris-HCl buffer solution containing 5\% DMSO as solvent).

Data for Co13. Yield: 80.5\%. Elemental analysis calcd (\%) for $\mathrm{C}_{32} \mathrm{H}_{24} \mathrm{Cl}_{4} \mathrm{CoN}_{4} \mathrm{O}_{2}$ : C 55.12, H 3.47, N 8.03; found: C 55.10, H 3.50, N 8.00. IR (KBr): $3445,1634,1483,1442,1363,1113,812,718 \mathrm{~cm}^{-1}$. ESI-MS m/z: 850.6 $[\mathrm{M}+2 \mathrm{DMSO}]^{+}$(Tris-HCl buffer solution containing 5\% DMSO as solvent).

Data for Co14. Yield: 75.5\%. Elemental analysis calcd (\%) for $\mathrm{C}_{30} \mathrm{H}_{16} \mathrm{CoI}_{4} \mathrm{~N}_{4} \mathrm{O}_{2}$ : C 34.95, $\mathrm{H}$ 1.56, N 5.43; found: C 34.93, H 1.60, N 5.42. IR (KBr): 3401, 3051, 1533, 1434, 1373, 1349, 1249, 1206, 1102, 926, 844, 786, 730, 653, 559, $419 \mathrm{~cm}^{-1}$. ESI-MS m/z: $634.3[\mathrm{M}-(\mathrm{QL} 4)]^{+}$(Tris-HCl buffer solution containing 5\% DMSO as solvent).

Data for Co15. Yield: 68.9\%. Elemental analysis calcd (\%) for $\mathrm{C}_{30} \mathrm{H}_{20} \mathrm{CoI}_{4} \mathrm{~N}_{4} \mathrm{O}_{4}$ : C 33.77, $\mathrm{H}$ 1.89, N 5.25; found: C 33.74, H 1.92, N 5.23. IR (KBr): 3442, 1607, 1533, 1438, 1376, 1346, 1249, 1104, 1038, 922, 831, 736, 656, $560 \mathrm{~cm}^{-1}$. ESI-MS m/z: $670.4[\mathrm{M}-(\mathrm{QL} 4)]^{+}$(Tris-HCl buffer solution containing 5\% DMSO as solvent).

Data for Co16. Yield: 89.1\%. Elemental analysis calcd (\%) for $\mathrm{C}_{30} \mathrm{H}_{20} \mathrm{CoI}_{5} \mathrm{~N}_{4} \mathrm{O}_{2}$ : C 31.01, $\mathrm{H} 1.73, \mathrm{~N} 4.82$; found: $\mathrm{C} 31.00, \mathrm{H} 1.76, \mathrm{~N} 4.80$. IR (KBr): 3777, 3425, 1618, 1477, 1438, 1352, 1245, 1110, 820, 755, 653, 580 $\mathrm{cm}^{-1}$. ESI-MS m/z: $1034.4[\mathrm{M}+\mathrm{H}]^{+}$(Tris- $\mathrm{HCl}$ buffer solution containing 5\% DMSO as solvent).

Data for Co17. Yield: 78.8\%. Elemental analysis calcd (\%) for $\mathrm{C}_{30} \mathrm{H}_{14} \mathrm{Br}_{4} \mathrm{Cl}_{2} \mathrm{CoN}_{4} \mathrm{O}_{2}$ : C 39.51, H 1.55, N 6.14; found: C 39.48, H 1.59, N 6.12. IR (KBr): 3439, 1615, 1534, 1439, 1378, 1348, 1206, 1105, 927, 839, 792, 735, 656, 558, $418 \mathrm{~cm}^{-1}$. ESI-MS m/z: $702.4[\mathrm{M}-(\mathrm{QL} 4)]^{+}$(Tris-HCl buffer solution containing 5\% DMSO as solvent). 
Data for Co18. Yield: 80.3\%. Elemental analysis calcd (\%) for $\mathrm{C}_{30} \mathrm{H}_{14} \mathrm{Cl}_{2} \mathrm{CoI}_{4} \mathrm{~N}_{4} \mathrm{O}_{2}$ : C 32.76, H 1.28, N 5.09; found: C 32.75, H 1.32, N 5.08. IR (KBr): 3412, 1567, 1494, 1455, 1377, 1317, 1247, 1085, 955, 832, 784, 733,

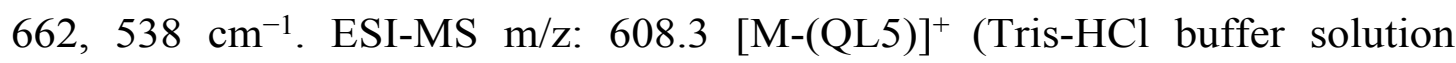
containing 5\% DMSO as solvent).

\section{Materials and methods}

The X-Ray crystallography were according to Sheldrick, et al. ${ }^{1}$ In addition, the detailed anti-tumor mechanism steps of Co1-Co18 were according reported previously methods. $^{2-10}$

\section{Animale Used}

The male and female KM mice, 5-6 weeks old, weight 20-23 g, six-week-old athymic BALB/cA nu/nufemale mice (18-20 g) were purchased from Guangxi Medical University Laboratory Animal Centre (Guangxi, China), the Acute toxicity studies and in vivo antitumor study were carried out in there. Animals were housed at a sterile environment with conditions of constant photoperiod (12 h light/ $12 \mathrm{~h}$ dark at $23-24{ }^{\circ} \mathrm{C}$ and $65-85 \%$ humidity).

In addition, the HeLa xenograft mouse models were purchased from Beijing HFK Bioscience Co., Ltd (Beijing, China, approval No. SCXK 2014-004). The animal procedures were approved by the Institute of Radiation Medicine Chinese Academy of Medical Sciences (Tian Jin, China, approval No. SYXK 2014-0002). And all of the experimental procedures were carried out in accordance with the NIH Guidelines for the Care and Use of Laboratory Animals. Animal experiments were approved by the Animal Care and Use Committee of Institute of Radiation Medicine Chinese Academy of Medical Sciences. In addition, statistical analysis and abbreviations used have been reported.

\section{Anticancer Activity toward HeLa in Vivo}

The HeLa cells were harvested and injected subcutaneously into the right flank of nude mice with $5 \times 10^{6}$ cells in $200 \mu \mathrm{L}$ of serum-free medium. When the xenograft tumor growth to the volume about $1000 \mathrm{~mm}^{3}$, the mice were killed and the tumor tissue were cut into about $1.5 \mathrm{~mm}^{3}$ small pieces, and then transplanted into the right flank of female nude mice, When tumors reach a volume of $100-300 \mathrm{~mm}^{3}$ on all mice, the mice were randomized into vehicle control and treatment groups ( $n=6 /$ group), 
received the following treatments: (a) control, 5.0\% v/v DMSO/saline vehicle, (b) Co7 at dose $2.0 \mathrm{mg} / \mathrm{kg}$ every two day (5.0\% v/v DMSO/saline). The tumor volumes were determined every three days by measuring length $(l)$ and width $(w)$ and calculating volume, tumor volume and inhibition of tumor growth were calculated using formulas 1-3:

Tumor volume: $\mathrm{V}=\left(\mathrm{w}^{2} \times 1\right) / 2$

The tumor relative increment rate: $\mathrm{T} / \mathrm{C}(\%)=\mathrm{T}_{\mathrm{RTV}} / \mathrm{C}_{\mathrm{RTV}} \times 100 \%$

inhibition of tumor growth: $\operatorname{IR}(\%)=\left(\mathrm{W}_{\mathrm{c}}-\mathrm{W}_{\mathrm{t}}\right) / \mathrm{W}_{\mathrm{c}} \times 100 \%$

Where $\mathrm{w}$ and 1 mean the shorter and the longer diameter of the tumor respectively; $\mathrm{T}_{\mathrm{RTV}}$ and $\mathrm{C}_{\mathrm{RTV}}$ was the RTV of treated group and control group respectively. (RTV: relative tumor volume, $\left.\mathrm{RTV}=\mathrm{V}_{\mathrm{t}} / \mathrm{V}_{0}\right) ; \mathrm{W}_{\mathrm{t}}$ and $\mathrm{W}_{\mathrm{c}}$ mean the average tumor weight of complex-treated and vehicle controlled group respectively.

\section{Statistical Analysis}

The experiments have been repeated from three to five times, and the results obtained are presented as means \pm standard deviation (SD). Significant changes were assesses by using Student's $t$ test for unpaired data, and $p$ values of $<0.05$ were considered significant.

\section{Abbreviations}

SD, standard deviation; TBS, Tris-HCl buffer; MTT, 3-(4,5-dimethylthiazol-2-yl)-2,5-diphenyltetrazolium bromide; TGI, tumor growth inhibition; PI, propidium iodide; MMP, mitochondrial membrane potential; JC-1, 5,5',6,6'-tetrachloro-1,1',3,3'-tetraethylbenzimidazolylcarbocyanine; IR, tumor growth inhibition rate. 


\section{REFERENCES}

(1) Sheldrick, G. M. SHELXS-97, Program for X-ray Crystal Structure Solution; University of Göttingen: Göttingen, Germany, 1997.

(2) Molphy, Z.; Prisecaru, A.; Slator, C.; Barron, N.; McCann, M.; Colleran, J.; Chandran, D.; Gathergood, N.; Kellett, A. Copper phenanthrene oxidative chemical nucleases. Inorg. Chem. 2014, 53, 5392-5404.

(3) Celli, G. B.; de Lange, T. DNA processing is not required for ATM-mediated telomere damage response after TRF2 deletion. Nat. Cell Biol. 2005, 7, 712-718.

(4) Krainz, T.; Gaschler, M. M.; Lim, C.; Sacher, J. R.; Stockwell, B. R.; Wipf, P. A Mitochondrial-Targeted Nitroxide Is a Potent Inhibitor of Ferroptosis. ACS Cent. Sci. 2016, 2, 653-659.

(5) Xun, Z.; Rivera-Sanchez, S.; Ayala-Pen, S.; Lim, J.; Budworth, H.; Skoda, E. M.; Robbins, P. D.; Niedernhofer, L. J.; Wipf, P.; McMurray, C. T. Targeting of XJB-5-131 to Mitochondria Suppresses Oxidative DNA Damage and Motor Decline in a Mouse Model of Huntington's Disease. Cell Rep. 2012, 2, 1137-1142.

(6) Wei, M. C.; Zong, W.-X.; Cheng, E. H. Y.; Lindsten, T.; Panoutsakopoulou, V.; Ross, A. J.; Roth, K. A.; MacGregor, G. R.; Thompson, C. B.; Korsmeyer, S. J. Proapoptotic BAX and BAK: a requisite gateway to mitochondrial dysfunction and death. Science 2001, 292, 727-730.

(7) Sinha, K.; Das, J.; Pal, P. B.; Sil, P. C. Oxidative stress: the mitochondria-dependent and mitochondria-independent pathways of apoptosis. Arch. Toxicol. 2013, 87, 1157-1180.

(8) Wang, J.-Q.; Zhang, P.-Y.; Ji, L.-N.; Chao, H. A ruthenium(II) complex inhibits tumor growth in vivo with fewer side-effects compared with cisplatin. J. Inorg. Biochem. 2015, 146, 89-96.

(9) Qin, Q.-P.; Wang, Z.-F.; Tan, M.-X.; Huang, X.-L.; Zou, H.-H.; Zou, B.-Q.; Shi, B.-B.; Zhang, S.-H. Complexes of lanthanides(III) with mixed 2, 2'-bipyridyl and 5, 7-dibromo-8-quinolinoline chelating ligands as a new class of promising anti-cancer agents, Metallomics, 2019, 11, 1005-1015.

(10) Meng, T.; Qin, Q.-P.; Chen, Z.-L.; Zou, H.-H.; Wang, K.; Liang, F.-P. Discovery of a high in vitro and in vivo antitumor activities of organometallic 
ruthenium(II)-arene complexes with 5,7-dihalogenated-2-methyl-8-quinolinol. Dalton Trans. 2019, 48, 5352-5360. 